Library, N.W. Bldg -

MAR 13 186?

NBS MONOGRAPH 19

\title{
Atomic Energy Levels In Crystals
}

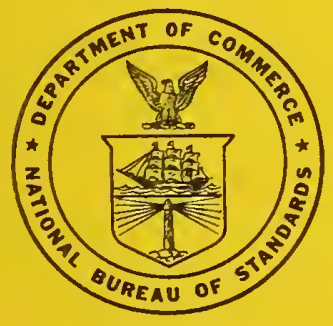

U.S. DEPARTMENT OF COMMERCE NATIONAL BUREAU OF STANDARDS 


\section{THE NATIONAL BUREAU OF STANDARDS}

\section{Functions and Activities}

The functions of the National Bureau of Standards are set forth in the Act of Congress, March 3, 1901, as amended by Congress in Public Law 619, 1950. These include the development and maintenance of the national standards of measurement and the provision of means and methods for making measurements consistent with these standards; the determination of physical constants and properties of materials; the development of methods and instruments for testing materials, devices, and structures; advisory services to government agencies on scientific and technical problems; invention and development of devices to serve special needs of the Government; and the development of standard practices, codes, and specifications. The work includes basic and applied research, development, engineering, instrumentation, testing, evaluation, calibration services, and various consultation and information services. Research projects are also performed for other government agencies when the work relates to and supplements the basic program of the Bureau or when the Bureau's unique competence is required. The scope of activities is suggested by the listing of divisions and sections on the inside of the back cover.

\section{Publications}

The results of the Bureau's work take the form of either actual equipment and devices or published papers. These papers appear either in the Bureau's own series of publications or in the journals of professional and scientific societies. The Bureau itself publishes three periodicals available from the Government Printing Office: The Journal of Research, published in four separate sections, presents complete scientific and technical papers; the Technical News Bulletin presents summary and preliminary reports on work in progress; and Basic Radio Propagation Predictions provides data for determining the best frequencies to use for radio communications throughout the world. There are also five series of nonperiodical publications: Monographs, Applied Mathematics Series, Handbooks, Miscellaneous Publications, and Technical Notes.

Information on the Bureau's publications can be found in NBS Circular 460, Publications of the National Bureau of Standards (\$1.25) and its Supplement (\$1.50), available from the Superintendent of Documents, Government Printing Office, Washington 25, D.C. 
UNITED STATES DEPARTMENT OF COMMERCE - Luther H. Hodges, Secretary NATIONAL BUREAU OF STANDARDS - A. V. Astin, Director

\section{Atomic Energy Levels in Crystals}

John L. Prather

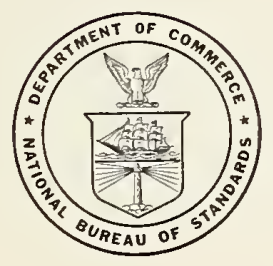

National Bureau of Standards Monograph 19

Issued February 24, 1961 


\section{Preface}

This present work had its inception in 1954, shortly after Professor G. H. Dieke of The Johns Hopkins University, with the support of the U.S. Atomic Energy Commission, initiated a program of experimental studies of the sharp line absorption spectra of crystals. The effort was directed principally to rare earth salt crystals, but some work was also done on the ruby and uranyl salts. Dr. Dieke suggested to the author that a theoretical study to accompany the experimental program would be desirable and encouraged the undertaking of such a study.

It rapidly became apparent on surveying the problem that the techniques of group theory would be of central importance in such a study. Early in 1955, Dr. Charles M. Herzfeld, then of the Naval Research Laboratory in Washington, addressed the Spectroscopy Seminar at Hopkins on the techniques of group theory with particular application to the paramagnetic resonance of salts of the iron group. This was the beginning of a continuing association with Dr. Herzfeld in the study of group theory, an association which has been a source of great personal and professional satisfaction to the author to the present time.

In August of 1955, through the courtesy of Dr. F. G. Brickwedde, then Chief of the Heat and Power Division of the National Bureau of Standards, and Dr. R. P. Hudson, Chief of the Cryogenic Physics Section of the Bureau, the author was appointed a Guest Worker at the Bureau, and office space was provided and the extensive library facilities of the Bureau made available. This appointment continued when Dr. Herzfeld was appointed Chief of the Heat Division.

The present work is largely an exhaustive collection of the results of group theory which apply to the theory of atomic energy levels in crystals, together with a careful, and detailed, but not especially abstract development of the general theory to permit an intelligent application of the results to experimental problems. Dr. Herzfeld, in collaboration with Professor Paul H. Meijer of The Catholic University of America, has written a review on the general subject "Group Theory and Crystal Field Theory", soon to be published, treating the subject from a more abstract viewpoint of modern algebra and concentrating on the fundamental aspects of the theory. This latter work and the present one form a complementary pair of works on the general topic. It is hoped that, together, they will meet the needs of most workers in the field. 


\section{Contents}

1. Introduction

2. The electrostatic field

2.1. Expansion in tesseral harmonics._. 3

2.2. Symmetry

2.3. Preferred coordinate systems

2.4. Cubic point groups_._.

3. Groups, characters, and representations

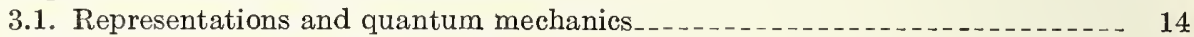

3.2. Rotations in three dimensions._.

3.3. Group theory and the free atom or ion

3.4. Group theory and the perturbed ion

3.5. Group characters and the rep $D_{J} \ldots \ldots \ldots \ldots$

3.6. Noncubic crystallographic groups

3.7. Noncubic double groups

3.8. Reps and characters

3.9. Infinite and cubic groups

3.10. Table of group characters

4. Degeneracy, selection rules, and polarization

4.1. Splitting of free ion levels

4.2. Kramers degeneracy

4.3. Orthogonal states and vanishing matrix elements

4.4. Selection rules and polarization.

5. Matrix elements_-_-_- 65

5.1. The reduced matrix element__. 65

5.2. Wigner coefficients

5.3. $L-S$ coupling and the one-electron approximation

5.4. Rare earth ground terms

6. Applications

6.1. General considerations_._. 74

6.2. A crystal field

6.3. The fluorescence spectrum of the terbium ion_._. 78

7. Bibliography

\section{List of Tables}

1. Normalized Legendre funetions.

2. Normalized tesseral harmonics._.

3. Structure of noncubic point groups

4. Symmetry elements in tesseral harmonies

5. Potential coefficients present for various symmetries

6. Transformation of tesseral harmonics.

7. A character table for the reps $D_{J} \ldots \ldots$

8. A table of group characters

9. Type and number of perturbed levels

10. Correlation tables_.

11. Reduction of the cubic groups

12. Multipole moment transformations

13. Selection rules_._.

14. Angular dependence of multipole components

15. Wigner coefficients.

16. Reduced matrix elements

17. A representative crystal field

18. Fluorescence spectrum of $\mathrm{TbCl}_{3} \cdot 6 \mathrm{H}_{2} \mathrm{O}$ 


\title{
Atomic Energy Levels in Crystals ${ }^{1,2}$
}

\author{
John L. Prather ${ }^{3}$
}

\begin{abstract}
Discrete energy levels observed within certain crystals are treated as due to perturbations of the energy levels of the free ion by an electrostatic field arising from the crystal lattice. The analytic procedures for determining the field from the charge configuration are given, and the resulting fields are classified according to their symmetry. After a general survey of group-theoretical ideas, the applicable groups are analyzed in detail, and characters appropriate for both integral and half-integral angular momenta of the free ion are tabulated. These are applied to the determination of the number and type of levels arising from a free ion level with $J \leq 8$. The results of this analysis are tabulated, as are the selection rules for electric dipole, magnetic dipole, and electric quadrupole transitions. Calculation of the perturbation matrix elements by the use of Wigner and Racah coefficients is discussed. Examples of the application of these several techniques to specific problems are given.
\end{abstract}

\section{Introduction}

In contrast to the sharp lines or resolvable bands characteristic of the optical absorption spectra of free atomic and molecular systems, such spectra for solids consist for the most part of broad absorption regions or bands without any resolvable structure. These spectra are therefore of little used for analysis of the details of the solids for which they are observed, revealing only general information such as the presence or absence of certain molecular groupings. Nevertheless, it was early recognized in the derelopment of optical spectroscopy that certain naturally occurring minerals did in fact show a characteristic line absorption spectrum. The earliest observations were on the crystals of salts of the iron group elements and of the rare earth elements, though later observations have shown similar spectra in crystals of all elements characterized by a partially filled inner shell of electrons. In this present work we shall be concerned primarily with the rare-earth spectra, though we shall sketch the application of the development to other possible cases.

The earliest data on these spectra, covering about 50 years before 1905 are reviewed by Kayser (see bibliography). Subsequent to the discovery of the Zeeman effect in 1895, J. Becquerel initiated an extensive investigation on the effects of magnetic fields on the absorption lines of natural rare earth crystals, their polarization, and particularly their rotation of the plane of polarization of light (Faraday effect). These measurements, carried out both at room temperature and at liquid air temperature, are summarized in his paper of 1908. In this same year, Becquerel and Onnes reported on the spectra and magnetic effects at liquid hydrogen temperatures, and measurements at liquid helium temperatures were finally reported in 1926 by Becquerel, Onnes, and de Haas. du Bois and Elias $(1908,1911)$ also

1 Based on a dissertation suhmitted to the Faculty of Philosophr of The Johns Hopkins University in partial fulfillment of the requirements for the degree Doctor of Philosophy.

Supported in part hy the U.S. Air Force, through the Air Force Office of Scientific Research of the Air Research and Development Command, under Contract No. CSO and A 650-56-21.

3 Gnest worker, formerly from The George Washington University, now कith Beloit College, Beloit, Wisc. reported work on chromium, cobalt, and uranium crystals as well as on the rare earths.

The year 1929 marked the beginning of an extensive program of investigation of these spectra at various temperatures by Spedding, Freed, and collaborators. The principal experimental advance over earlier work lay in the use of synthetic crystals of more definite composition than the natural crystals, which usually consisted of a mixture of rare earths of varying composition. Hence there had been little if any reliability in intercomparison of the results obtained from various crystals, even of the same nominal type. This was a considerable advance over the previous attempts at a systematic experimental approach to these spectra, though by today's standards even these crystals were not of satisfactory purity. These measurements were also much aided by continuing developments in the theory of these spectra, which we shall trace below.

All of these spectra show the same general pattern and behavior upon reducing the temperature. The absorption lines for various elements fall in to groups in several regions of the spectrumthe general wavelength regions where the lines fall depend on the rare earth involved, while details concerning the number of lines, the exact position, intervals, Zeeman effect, etc., depend on the negative ions involved and on the details of the crystal structure. In comparison with room temperature measurements, those at reduced temperatures show generally sharper lines, together with a slight overall shift of the groups to the red and increased separation of the lines within a group (Spedding and Bear, 1932). The number of lines also changes; certain "high temperature lines" disappear, while others appear or become more intense at lower temperatures. Such lines have also been observed in solutions of the ions, though these lines are generally broader than in the crystals.

Although there was some theoretical speculation on these spectra, no theoretically satisfactory contributions save the derivation of empirical energy level diagrams could be made prior to the advent of quantum mechanics. The first major 
contribution directly related to the rare earth spectra was the calculation by Hund (1925) of the room temperature paramagnetic susceptibility of the rare earth crystals on the assumption that the crystals consisted of an independent statistical assemblage of ions, characterized by an unfilled shell of $4 f$ electrons which became increasingly filled as one proceeded up the periodic table. The ground states of these ions were calculated on the assumption that Hund's rules for free atoms were valid, i.e., that of all the terms arising from the given $4 f^{n}$ configuration, that with the greatest multiplicity had the lowest energy, and of those of this greatest multiplicity, the largest $L$ value was lowest. For a given $L-S$ term, the resulting multiplet was taken as normal in the first half of the series (the $4 f$ shell being less than half filled) and as inverted in the last half of the series. The agreement with experiment was quite good, save in the cases of europium and samarium, and these two discrepancies were corrected by the second order calculations of Van Vleck and Frank (1929), Hund having considered only the lowest level of the ground multiplet as contributing to the susceptibility. This agreement with experiment not only gave evidence for the electronic structure of the rare earth ions in the crystals, but also suggested that the effects on the magnetic properties of the ion due to inclusion in the crystal were small in comparison with $k T$ at $300^{\circ} \mathrm{K}$ and also small in comparison with the multiplet splitting of the ground $L-S$ term.

It was perhaps natural to assume that the line spectra observed were similarly due to the spectra of the free ions, modified to some slight degree by their inclusion in the crystal, but the first suggestion to this effect was made by Brunetti and Ollano (1929), who considered the crystal as a perturbing electrostatic field on the free ion spectrum. This idea was also developed by Freed and Spedding (1929) in initiating their program of experimental observations noted above. The classic paper in these early years of the theory is that by Bethe (1929). Bethe pointed out that the details of the theoretical treatment depended on the strength of this external crystal field compared to the other interactions present (principally the Coulomb interaction of the electrons and the spin-orbit interaction), and that the symmetry alone of the external field, independent of its magnitude, would determine the number and type of levels arising from a given level of the free ion. These were specifically computed by Bethe for certain symmetries, emphasizing in the process the importance of group-theoretical ideas for such calculations, and the properties of one-electron wave functions under these same crystal fields were also calculated. These ideas were later extended to treat the Zeeman effect in crystals (Bethe, 1930). At about the same time, Kramers (1929, 1930) considering principally the Faraday effect, formulated the fundamental requirement that these electrostatic fields arising from the crystal must leave every level of an odd-electron system at least twofold degenerate.

Although it seemed clear that the idea of an electrostatic perturbation acting upon the levels of the free ion was the key to the problem, it was not at all certain what transitions were responsible for the lines actually observed. The situation was complicated by utter ignorance of the actual free ion spectrum, and even as recently as 1958 only that of CeIV is known (Lang, 1936). The earliest suggestions were that the transition involved a $4 f$ electron excited to either $5 d$ or $6 s$ (Spedding, 1931; Spedding, 1933). These were based principally on analogy with the selection rules applicable to free ions. However, the sharpness of the lines indicates a freedom from interaction with other ions which would not be expected from such levels (involving $5 d$ or $6 s$ ) having a fair probability of being at appreciable distances from the nucleus. Furthermore, the general intensity of the lines relative to the high concentration of ions in a solid yields transition probabilities corresponding to transitions forbidden in the usual type of atomic spectra (Van Vleck, 1937). For this reason, it is today generally considered that the lines come from transitions between terms arising from the ground $4 f^{n}$ configuration. This view is strengthened by the simplicity of such sharp line spectra in the salts of cerium and ytterbium (Dieke and Crosswhite, 1956) both of which have only one excited level from the ground configurations $4 f^{1}$ and $4 f^{13}$, respectively. Van Vleck also made some estimates of the relative probability of transitions due to electric quadrupole radiation, magnetic dipole radiation, electric dipole radiation arising from absence of a static center of symmetry in the crystal field (which destroys the characterization of levels as odd or even), and electric dipole radiation arising from destruction of a static center of symmetry by lattice vibrations. It was concluded that there was no clear quantitative reason to reject completely any of these as being not responsible for the lines observed.

During this period (the 1930's) detailed calculations were made by Penney and Schlapp (1932, Schlapp and Penney) based on the assumption of a cubic crystal field, adjusting the parameters to fit observed magnetic susceptibility data. Although good agreement with experiment in this regard was obtained, it is in marked disagreement with the spectral data. This may be ascribed to the insensitivity of susceptibility data (a statistical average over all ions present) to details of the crystal field. The same objection also applies to attempts to derive the crystal field from specific heat data (Ahlberg et al., 1937). It is now known that the symmetry of the cubic system is too high to account for the spectroscopic observations.

Apart from theoretical considerations of the rare earth spectra alone, several general theoretical contributions have been made which are applicable in major part to the determination of the energy 
levels of rare earth ions in crystals. Since these will be referred to in detail later in this work, we shall merely mention them for general background at this point. The general applicability of group theory to problems of atomic structure has been emphasized by Weyl (1928), clarified by Eckart (1930), and treated at length by Wigner (1931). The calculations of Bethe (1929) for certain symmetry groups were extended by Wigner (1930) to other symmetries, though this was in connection with problems of molecular vibrations. The monumental work of Condon and Shortley (1935) is invaluable for the study of the free ion spectrum.

While experimental data on many different crystals were collected by many observers, using many different techniques (absorption spectra, Zeeman effect, fluorescence spectra, magnetic measurements, specific heat data) during the following years, the next major advance was the application of paramagnetic resonance techniques to the rare earth crystals. A general review of this work may be found in the paper by Bleaney and Stevens (1953), while additional data are given in the supplementary article by Bowers and Owen (1955). This method has the advantage of giving data of high accuracy and resolution-its major limitation is in the fact that with rare exceptions only data on the ground level in the crystal are obtained.

The next major advance in the general theory was the work of Racah $(1942,1942 a, b)$ on complex spectra, which provided very powerful general techniques for the solution of problems in manyelectron systems. His results have been made applicable to specific numerical calculations by the recent appearance of numerical tables by Biedenharn (1952), Simon (1954), and Simon et al. (1954). While the procedures of Racah have been used in several fields, and their applicability to the problem of rare earth spectra in crystals has been mentioned by Elliott and Stevens (1953), no widespread use of them has been made in crystal spectra problems. The general work of Bethe (1929) on the applications of group theory to the problem has subsequently been applied by various authors to special cases, but no general overall survey of the possibilities has been given. The results have been obtained by Hellwege (1948, 1948a-d) without the explicit use of group theory, but much elegance and conciseness is lost thereby, and the results are presented in a rather cumbersome form. A general survey of these and many other aspects of the problem by Fick and Joos (1957) has recently appeared.
Recent renewed interest in these spectra and the associated properties of the ions has generated a requirement for a comprehensive survey and development of the theoretical techniques required for an analysis of such spectra. While the theory in its most general terms is known, its applications in the past have either been concerned with a particular salt or ion, or have used only a portion of the theoretical equipment available, are widely scattered through the literature, and their usefulness is limited by differences in conventions of sign, phase, normalization, notation, etc. It is the purpose of this paper to provide a comprehensive theoretical approach to the analysis of these spectra in a form particularly adapted to the requirements of the experimental workers. In the interest of meeting this aim, mathematical rigor and long purely algebraic manipulations have for the most part been omitted, except where necessary for an understanding of the concepts and procedures involved. It is hoped that it will be of value to experimentalists in the field who have had only a general background in the ordinary theory of the atomic spectra of free atomic systems.

Since the general problem is that of computing the effect on the free ion of the electrostatic perturbations arising from the crystal lattice, we begin by considering in section 2 the development of this potential in a power series of tesseral harmonics, in explicit terms of the charge and position of the ions giving rise to the field. An important property of such fields is their symmetry, and this will be discussed in detail. In section 3 , we shall consider the connection between the theory of groups and quantum mechanics. We begin by sketching the group-theoretical ideas associated with the problem of the free atom. This leads naturally into those modifications caused by the inclusion of the ion in the crystal lattice. The properties of the groups involved in the present problem are then considered in detail. In section 4 , we present explicitly the results of the considerations of section 3 to the degeneracy of levels, selection rules and polarization of lines in the spectrum of the crystal. In section 5, we consider the calculation of the matrix elements of the perturbing electrostatic potential. Finally, we conclude in section 6 by a review of the various factors influencing the experimentally observed spectra, correlated with the spectra of the free ion, and some examples of the application of the techniques to specific problems.

\section{The Electrostatic Field}

\subsection{Expansion in Tesseral Harmonics}

Let us consider, in a given frame of reference, an ion with a charge $q$ located at the point described in spherical coordinates as $(R, \alpha, \beta)$. The potential at the point $(r, \theta, \phi)$ may be expressed as a series of Legendre polynomials in the variable $\cos \gamma$, where $\gamma$ is the angle between the directions given by $\alpha, \beta$, and $\theta, \phi$. The unit vectors in the two directions are $\sin \alpha \cos \beta \mathbf{i}+\sin \alpha \sin \beta \mathbf{j}+\cos \alpha \mathbf{k}$, and $\sin \phi \cos \phi \mathbf{i}+\sin \theta \sin \phi \mathbf{j}+\cos \theta \mathbf{k}$, respectively. Thus, $\cos \gamma$ is the scalar product of the two unit vectors and $\cos \gamma=\sin \theta \sin \alpha \cos \beta \cos \phi+\sin \theta \sin \alpha$ $\sin \beta \sin \phi+\cos \theta \cos \alpha=\sin \alpha \sin \theta \cos (\phi-\beta)+$ 
$\cos \theta \cos \alpha$. The potential is given, for $r \leq R$, by

$$
V=q \sum_{N=0}^{\infty} \frac{r^{N}}{R^{N+1}} P_{N}(\cos \gamma)
$$

The total potential is the sum of similar contributions from each ion of the crystal lattice. In the above expression, the contribution of each ion is described with reference to a different axis, and it is convenient to refer each contribution to the same axis, which we choose to be the $z$-axis of the given frame of reference.

This may be accomplished through the use of the addition theorem of spherical harmonics, which expresses $P_{N}(\cos \gamma)$ in terms of $\alpha, \beta, \theta$, and $\phi$ (Stratton, 1941).

$P_{N}(\cos \gamma)=P_{N}(\cos \alpha) P_{N}(\cos \theta)+2 \sum_{M=1}^{N} \frac{(N-M) !}{(N+M) !}$

$$
P_{N}^{M}(\cos \alpha) P_{N}^{M}(\cos \theta) \cos M(\phi-\beta) .
$$

The potential due to the $i$-th charge can then be be put into the form

$$
\begin{aligned}
V_{i}=q_{i} \sum_{N=0}^{\infty} \frac{r^{N}}{R_{i}^{N+1}}\left[P_{N}\left(\cos \alpha_{i}\right) P_{N}(\cos \theta)\right. \\
+2 \sum_{M=1}^{N} \frac{(N-M) !}{(N+M) !} P_{N}^{M}\left(\cos \alpha_{i}\right) P_{N}^{M}(\cos \theta) \\
\left.\quad\left(\cos M \phi \cos M \beta_{i}+\sin M \phi \sin M \beta_{i}\right)\right]
\end{aligned}
$$

The total potential may then be obtained by summing over all charges in the lattice.

As used above, the $P_{N}^{M}(\cos \theta)$ are not normalized in the quantum-mechanical sense, since

$$
\begin{aligned}
\int_{-1}^{1} P_{N}^{M}(\cos \theta) P_{K}^{L}(\cos \theta) & d(\cos \theta) \\
& =\delta_{N, K} \delta_{M, x} \frac{2}{2 N+1} \frac{(N+M) !}{(N-M) !}
\end{aligned}
$$

and it is consequently convenient to define

$$
\theta_{N}^{M}=\sqrt{\frac{2 N+1}{2} \cdot \frac{(N-M) !}{(N+M) !}} P_{N}^{M}(\cos \theta) .
$$

Tabulated functions $\theta_{N}^{M}$ for $N$ up to and including 6 are given in table 1 . Similarly, the functions $\frac{1}{\sqrt{\pi}} \sin M \phi, \frac{1}{\sqrt{\pi}} \cos M \phi$, and $\frac{1}{\sqrt{2 \pi}}$ constitute an orthonormal set of functions over the interval $[0,2 \pi]$ in the variable $\phi$. We shall define the following tesseral harmonics, which are functions of position on the surface of the unit sphere, and may be evaluated either from $\theta, \phi$, or from $x, y$,
TABLE 1. Normalized Legendre functions

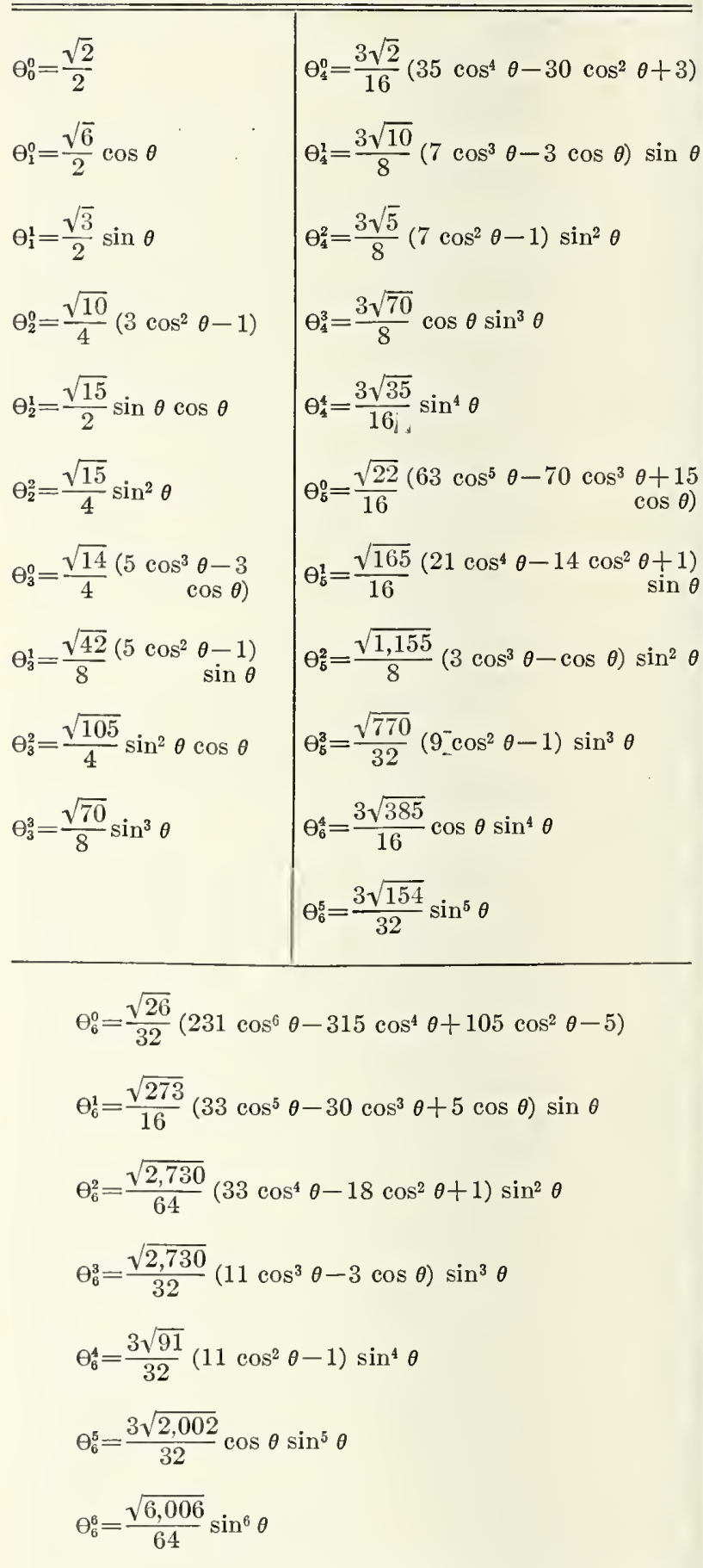

and $z$. They are given as functions of $x, y$, and $z$ in table 2:

$$
\begin{aligned}
& \mathrm{S}_{N}^{M}=\Theta_{N}^{M} \frac{\sin M \phi}{\sqrt{\pi}}, \mathrm{S}_{N}^{0}=0, \\
& \mathrm{C}_{N}^{M}=\Theta_{N}^{M} \frac{\cos M \phi}{\sqrt{\pi}}, \quad \mathrm{C}_{N}^{0}=\frac{1}{\sqrt{2 \pi}} \Theta_{N}^{0} .
\end{aligned}
$$


TABLE 2. Normalized tesseral harmonics

\begin{tabular}{l|l}
\hline \hline $\mathrm{C}_{1}^{0}=\frac{\sqrt{6}}{2} \cdot \frac{1}{\sqrt{2 \pi}} \cdot \frac{z}{r}$ & $\mathrm{C}_{3}^{0}=\frac{\sqrt{14}}{4} \cdot \frac{1}{\sqrt{2 \pi}} \cdot \frac{2 z^{3}-3 x^{2} z-3 y^{2} z}{r^{3}}$ \\
$\mathrm{C}_{1}^{1}=\frac{\sqrt{3}}{2} \cdot \frac{1}{\sqrt{\pi}} \cdot \frac{x}{r}$ & $\mathrm{C}_{3}^{1}=\frac{\sqrt{42}}{8} \cdot \frac{1}{\sqrt{\pi}} \cdot \frac{4 z^{2} x-x^{3}-x y^{2}}{r^{3}}$ \\
$\mathrm{~S}_{1}^{1}=\frac{\sqrt{3}}{2} \cdot \frac{1}{\sqrt{\pi}} \cdot \frac{y}{r}$ & $\mathrm{~S}_{3}^{1}=\frac{\sqrt{42}}{8} \cdot \frac{1}{\sqrt{\pi}} \cdot \frac{4 z^{2} y-x^{2} y-y^{3}}{r^{3}}$ \\
$\mathrm{C}_{2}^{0}=\frac{\sqrt{10}}{4} \cdot \frac{1}{\sqrt{2 \pi}} \cdot \frac{2 z^{2}-x^{2}-y^{2}}{r^{2}}$ & $\mathrm{C}_{3}^{2}=\frac{\sqrt{105}}{4} \cdot \frac{1}{\sqrt{\pi}} \cdot \frac{z x^{2}-z y^{2}}{r^{3}}$ \\
$\mathrm{C}_{2}^{1}=\frac{\sqrt{15}}{2} \cdot \frac{1}{\sqrt{\pi}} \cdot \frac{x z}{r^{2}}$ & $\mathrm{~S}_{3}^{2}=\frac{\sqrt{105}}{4} \cdot \frac{1}{\sqrt{\pi}} \cdot \frac{2 x y z}{r^{3}}$ \\
$\mathrm{~S}_{2}^{1}=\frac{\sqrt{15}}{2} \cdot \frac{1}{\sqrt{\pi}} \cdot \frac{y z}{r^{2}}$ & $\mathrm{C}_{3}^{3}=\frac{\sqrt{70}}{8} \cdot \frac{1}{\sqrt{\pi}} \cdot \frac{x^{3}-3 x y^{2}}{r^{3}}$ \\
$\mathrm{C}_{2}^{2}=\frac{\sqrt{15}}{4} \cdot \frac{1}{\sqrt{\pi}} \cdot \frac{x^{2}-y^{2}}{r^{2}}$ & $\mathrm{~S}_{3}^{3}=\frac{\sqrt{70}}{8} \cdot \frac{1}{\sqrt{\pi}} \cdot \frac{3 x^{2} y-y^{3}}{r^{3}}$ \\
$\mathrm{~S}_{2}^{2}=\frac{\sqrt{15}}{4} \cdot \frac{1}{\sqrt{\pi}} \cdot \frac{2 x y}{r^{2}}$ & \\
\hline
\end{tabular}

$\mathrm{C}_{\mathbf{8}}^{0}=\frac{3 \sqrt{2}}{16} \cdot \frac{1}{\sqrt{2 \pi}} \cdot \frac{8 z^{4}+3 x^{4}+3 y^{4}-24 x^{2} z^{2}-24 y^{2} z^{2}+6 x^{2} y^{2}}{r^{4}}$

$\mathrm{C}_{\mathrm{i}}^{1}=\frac{3 \sqrt{10}}{8} \cdot \frac{1}{\sqrt{\pi}} \cdot \frac{\left(4 z^{2}-3 x^{2}-3 y^{2}\right)(x z)}{r^{4}}$

$\mathrm{S}_{4}^{1}=\frac{3 \sqrt{10}}{8} \cdot \frac{1}{\sqrt{\pi}} \cdot \frac{\left(4 z^{2}-3 x^{2}-3 y^{2}\right)(y z)}{r^{4}}$

$\mathbf{C}_{4}^{2}=\frac{3 \sqrt{5}}{8} \cdot \frac{1}{\sqrt{\pi}} \cdot \frac{\left(6 z^{2}-x^{2}-y^{2}\right)\left(x^{2}-y^{2}\right)}{r^{4}}$

$\mathrm{S}_{4}^{2}=\frac{3 \sqrt{5}}{8} \cdot \frac{1}{\sqrt{\pi}} \cdot \frac{\left(6 z^{2}-x^{2}-y^{2}\right)(2 x y)}{r^{4}}$

$\mathrm{C}_{4}^{3}=\frac{3 \sqrt{70}}{8} \cdot \frac{1}{\sqrt{\pi}} \cdot \frac{\left(x^{2}-3 y^{2}\right)(x z)}{r^{4}}$

$\mathbf{S}_{4}^{3}=\frac{3 \sqrt{70}}{8} \cdot \frac{1}{\sqrt{\pi}} \cdot \frac{\left(3 x^{2}-y^{2}\right)(y z)}{r^{4}}$

$C_{4}^{4}=\frac{3 \sqrt{35}}{16} \cdot \frac{1}{\sqrt{\pi}} \cdot \frac{x^{4}-6 x^{2} y^{2}+y^{4}}{r^{4}}$

$\mathbf{S}_{4}^{4}=\frac{3 \sqrt{35}}{16} \cdot \frac{1}{\sqrt{\pi}} \cdot \frac{4 x y\left(x^{2}-y^{2}\right)}{1^{4}}$

$C_{5}^{0}=\frac{\sqrt{22}}{16} \cdot \frac{1}{\sqrt{2 \pi}} \cdot \frac{8 z^{5}-40 z^{3}\left(x^{2}+y^{2}\right)+15\left(x^{2}+y^{2}\right)^{2} z}{r^{5}}$

$\mathrm{C}_{5}=\frac{\sqrt{165}}{16} \cdot \frac{1}{\sqrt{\pi}} \cdot \frac{\left(8 z^{4}+x^{4}+y^{4}+2 x^{2} y^{2}-12 x^{2} z^{2}-12 y^{2} z^{2}\right) x}{r^{5}}$

$\mathrm{S}_{5}^{1}=\frac{\sqrt{165}}{16} \cdot \frac{1}{\sqrt{\pi}} \cdot \frac{\left(8 z^{4}+x^{4}+y^{4}+2 x^{2} y^{2}-12 x^{2} z^{2}-12 y^{2} z^{2}\right) y}{r^{5}}$

$551664-61-2$
$\mathrm{C}_{5}^{2}=\frac{\sqrt{1,155}}{8} \cdot \frac{1}{\sqrt{\pi}} \cdot \frac{\left(2 z^{2}-x^{2}-y^{2}\right)\left(x^{2}-y^{2}\right) z}{r^{5}}$

$\mathrm{S}_{5}^{2}=\frac{\sqrt{1,155}}{8} \cdot \frac{1}{\sqrt{\pi}} \cdot \frac{\left(2 z^{2}-x^{2}-y^{2}\right)(2 x y z)}{r^{5}}$

$\mathbf{C}_{5}^{3}=\frac{\sqrt{770}}{32} \cdot \frac{1}{\sqrt{\pi}} \cdot \frac{\left(8 z^{2}-x^{2}-y^{2}\right)\left(x^{3}-3 x y^{2}\right)}{r^{5}}$

$\mathrm{S}_{5}^{3}=\frac{\sqrt{770}}{32} \cdot \frac{1}{\sqrt{\pi}} \cdot \frac{\left(8 z^{2}-x^{2}-y^{2}\right)\left(3 x^{2} y-y^{3}\right)}{r^{5}}$

$C_{5}^{4}=\frac{3 \sqrt{385}}{16} \cdot \frac{1}{\sqrt{\pi}} \cdot \frac{\left(x^{4}-6 x^{2} y^{2}+y^{4}\right) z}{r^{5}}$

$\mathbf{S}_{5}^{4}=\frac{3 \sqrt{385}}{16} \cdot \frac{1}{\sqrt{\pi}} \cdot \frac{\left(x^{2}-y^{2}\right)(4 x y z)}{r^{5}}$

$\mathrm{C}_{5}^{5}=\frac{3 \sqrt{154}}{32} \cdot \frac{1}{\sqrt{\pi}} \cdot \frac{x^{5}-10 x^{3} y^{2}+5 x y^{4}}{r^{5}}$

$\mathrm{S}_{5}^{5}=\frac{3 \sqrt{154}}{32} \cdot \frac{1}{\sqrt{\pi}} \cdot \frac{y^{5}-10 y^{3} x^{2}+5 y x^{4}}{r^{5}}$

$C_{8}^{0}=\frac{\sqrt{26}}{32} \cdot \frac{1}{\sqrt{2 \pi}} \cdot \frac{16 z^{6}-120\left(x^{2}+y^{2}\right) z^{4}+90\left(x^{2}+y^{2}\right)^{2} z^{2}-5\left(x^{2}+y^{2}\right)^{3}}{r^{6}}$

$C_{6}^{1}=\frac{\sqrt{273}}{16} \cdot \frac{1}{\sqrt{\pi}} \cdot \frac{\left[8 z^{5}-20 z^{3}\left(x^{2}+y^{2}\right)+5 z\left(x^{2}+y^{2}\right)^{2}\right] x}{r^{6}}$

$\mathrm{S}_{6}^{1}=\frac{\sqrt{273}}{16} \cdot \frac{1}{\sqrt{\pi}} \cdot \frac{\left[8 z^{5}-20 z^{3}\left(x^{2}+y^{2}\right)+5 z\left(x^{2}+y^{2}\right)^{2}\right] y}{r^{6}}$

$\mathrm{C}_{6}^{2}=\frac{\sqrt{2,730}}{64} \cdot \frac{1}{\sqrt{\pi}} \cdot \frac{\left[16 z^{4}-16\left(x^{2}+y^{2}\right) z^{2}+\left(x^{2}+y^{2}\right)^{2}\right]\left(x^{2}-y^{2}\right)}{r^{6}}$

$\mathbf{S}_{6}^{2}=\frac{\sqrt{2,730}}{64} \cdot \frac{1}{\sqrt{\pi}} \cdot \frac{\left[16 z^{4}-16\left(x^{2}+y^{2}\right) z^{2}+\left(x^{2}+y^{2}\right)^{2}\right](2 x y)}{r^{6}}$

$\mathrm{C}_{6}^{3}=\frac{\sqrt{2,730}}{32} \cdot \frac{1}{\sqrt{\pi}} \cdot \frac{\left(8 z^{2}-3 x^{2}-3 y^{2}\right)\left(x^{2}-3 y^{2}\right)(x z)}{r^{6}}$

$\mathrm{S}_{0}^{3}=\frac{\sqrt{2,730}}{32} \cdot \frac{1}{\sqrt{\pi}} \cdot \frac{\left(8 z^{2}-3 x^{2}-3 y^{2}\right)\left(3 x^{2}-y^{2}\right)(y z)}{r^{6}}$

$\mathrm{C}_{6}^{1}=\frac{3 \sqrt{91}}{32} \cdot \frac{1}{\sqrt{\pi}} \cdot \frac{\left(10 z^{2}-x^{2}-y^{2}\right)\left(x^{4}-6 x^{2} y^{2}+y^{4}\right)}{r^{6}}$

$\mathbf{S}_{6}^{4}=\frac{3 \sqrt{91}}{32} \cdot \frac{1}{\sqrt{\pi}} \cdot \frac{\left(10 z^{2}-x^{2}-y^{2}\right)\left(4 x^{3} y-4 x y^{3}\right)}{r^{6}}$

$\mathrm{C}_{8}^{5}=\frac{3 \sqrt{2,002}}{32} \cdot \frac{1}{\sqrt{\pi}} \cdot \frac{\left(x^{5}-10 x^{3} y^{2}+5 x y^{4}\right) z}{r^{6}}$

$\mathbf{S}_{6}^{5}=\frac{3 \sqrt{2,002}}{32} \cdot \frac{1}{\sqrt{\pi}} \cdot \frac{\left(y^{5}-10 y^{3} x^{2}+5 y x^{4}\right) z}{r^{6}}$

$\mathbf{C}_{6}^{6}=\frac{\sqrt{6,006}}{64} \cdot \frac{1}{\sqrt{\pi}} \cdot \frac{x^{6}-15 x^{4} y^{2}+15 x^{2} y^{4}-y^{6}}{r^{6}}$

$\mathbf{S}_{6}^{6}=\frac{\sqrt{6,006}}{64} \cdot \frac{1}{\sqrt{\pi}} \cdot \frac{\left(3 x^{2}-y^{2}\right)\left(x^{2}-3 y^{2}\right)(2 x y)}{r^{6}}$ 
With these substitutions, the potential may now be written in the form

$$
V=\sum_{N=0}^{\infty} r^{N} \sum_{M=0}^{N}\left(C_{N}^{M} C_{N}^{M}+S_{N}^{M} S_{N}^{M}\right)
$$

where the coefficients $C_{N}^{M}$ and $S_{N}^{M}$ depend on the size and location of the neighboring ions and are given explicitly by

$$
\begin{aligned}
& C_{N}^{M}=\frac{4 \pi}{2 N+1} \sum_{i} \frac{q_{i}}{R_{i}^{N+1}} C_{N}^{M}\left(\alpha_{i}, \beta_{i}\right) \\
& S_{N}^{M}=\frac{4 \pi}{2 N+1} \sum_{i} \frac{q_{i}}{R_{i}^{N+1}} S_{N}^{M}\left(\alpha_{i}, \beta_{i}\right)
\end{aligned}
$$

We recall here that the $i$-th ion, charge $q_{i}$, is located in spherical coordinates at $\left(R_{i}, \alpha_{i}, \beta_{i}\right)$. The terms $\mathbf{C}_{N}^{M}\left(\alpha_{i}, \beta_{i}\right)$ and $\mathbf{S}_{N}^{M}\left(\alpha_{i}, \beta_{i}\right)$ represent the quantities (2.5) evaluated for the several pairs of angles involved in the summation.

We shall also have occasion to utilize the complex form of (2.5), defining

$$
\mathrm{Y}_{N}^{M}=\frac{(-1)^{M}}{\sqrt{2}}\left(\mathbf{C}_{N}^{M}+i \mathrm{~S}_{N}^{M}\right), \mathbf{Y}_{N}^{-M}=\frac{1}{\sqrt{2}}\left(\mathbf{C}_{N}^{M}-i \mathrm{~S}_{N}^{M}\right) .
$$

and

$$
\mathrm{Y}_{N}^{0}=\mathrm{C}_{N}^{0}
$$

This will similarly require, in lieu of $(2.7 \mathrm{a}, \mathrm{b})$ the coefficients

$$
Y_{N}^{M}=\frac{(-1)^{M}}{\sqrt{2}}\left(C_{N}^{M}-i S_{N}^{M A}\right), Y_{N}^{-M}=\frac{1}{\sqrt{2}}\left(C_{N}^{M}+i S_{N}^{M}\right) .
$$

This expression (2.6) for the potential diverges for $r>R$. As we shall see later, however, we shall require only terms for $N$ up to and including 6 , and we shall assume that the wave function of the free ion vanishes sufficiently rapidly for large $r$ that negligible error is made by integrating to infinite distances.

\subsection{Symmetry}

The effects of the electrostatic field on the free ion spectrum may be separated into two categories: first, those depending on the qualitative nature of the field (its shape or symmetry), which is expressed through the mere presence or absence of certain of the $C_{N}^{M}$ or $S_{N}^{M}$; and second, the quantitative details which depend on the sign and magnitude of the $C_{N}^{M}$ and $S_{N}^{M}$. It is convenient to consider separately these two characteristics of the field.

There are several standard and equivalent techniques for describing the symmetry of the electrostatic field. For example, given an arbitrary point $(x, y, z)$ of the field, one may indicate the coordinates of all points having the same value of the potential, such as $(x,-y, z),(-x, y, z)$, $(-x,-y, z)$. Another widely used scheme is to indicate by a symbolic notation the operations which convert a given initial point into one of equal potential. Thus, in the example just given, the operations are respectively a reflection in the $x-z$ plane, a reflection in the $y-z$ plane, and a rotation of $\pi$ about the $z$ axis, and may be designated as $\sigma_{x z}, \sigma_{y z}$, and $\boldsymbol{C}_{2}$. Finally, for a given point $(x, y, z)$ one may give those $3 \times 3$ matrices which transform the original point into one of equal potential. If one is thoroughly familiar with the notation and properties of the various rotations, reflections, and the inversion, there is no reason to prefer one of these to the other. If one does not have this familiarity, the use of the matrices has the advantage that the effect of two successive operations can be computed by matrix multiplication without recourse to geometric arguments. Thus, if $\mathbf{r}^{\prime}=\mathbf{A r}$ and $\mathbf{r}^{\prime \prime}=\mathbf{B r}$ for all $\mathbf{r}$, then $\mathbf{r}^{\prime \prime \prime}=\mathrm{ABr}$ is also a point of equal potential as is $\mathbf{r}^{\prime \prime \prime \prime}=\mathbf{B A r}$. For the problem to be considered here, there are only a few basic matrices which must be considered. These, together with their symbolic notation (Schönflies, 1923) are:

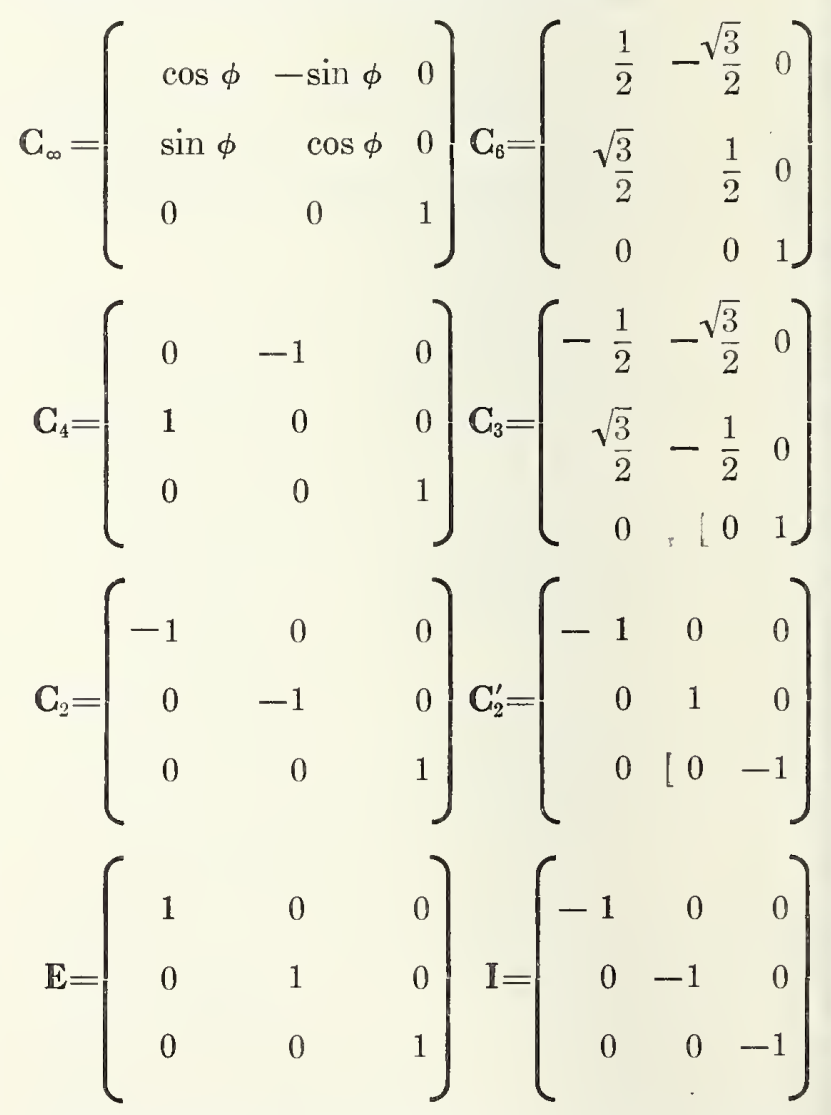

Thus, the point (or vector) $\mathbf{r}=(x, y, z)$ under the operation $\mathbf{C}_{\infty}$ becomes

$$
\mathbf{r}^{\prime}=(x \cos \phi-y \sin \phi, x \sin \phi+y \cos \phi, z) .
$$

We see that this is equivalent to a rotation of the vector about the $z$-axis by an angle $\phi$, counterclockwise (i.e., by $+\phi$ in terms of the right-hand 
screw convention) when looking toward the origin along the positive $z$-axis. This may also be interpreted as a new description of the old point $(x, y$, $z$ ) in terms of a new coordinate system where the coordinate system has been rotated by the angle $\phi$ in the opposite sense. In figure 1, we have initially (in two dimensions only) the vector $\mathbf{r}$ in the

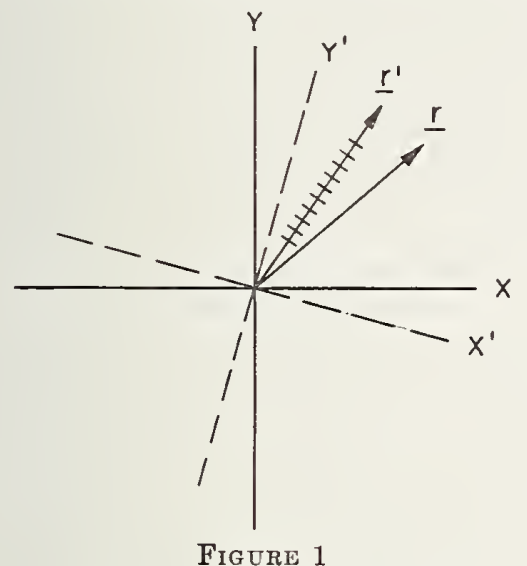

$x, y$ frame. The operation $\mathbf{C}_{\infty}$ will then give $\mathbf{r}^{\prime}$ in the $x, y$ frame. However, this is clearly the same as $\mathbf{r}$ described in the rotated frame $x^{\prime}, y^{\prime}$. We shall use both interpretations in the present work.

$$
\begin{aligned}
& \mathbf{I C}_{2}=\left(\begin{array}{rrr}
1 & 0 & 0 \\
0 & 1 & 0 \\
0 & 0 & -1
\end{array}\right)=\boldsymbol{\sigma}_{h} \\
& \mathbf{I C}_{3}^{2}=\left(\begin{array}{rrr}
\frac{1}{2} & -\frac{3}{2} & 0 \\
\frac{3}{2} & \frac{1}{2} & 0 \\
0 & 0 & -1
\end{array}\right)=\boldsymbol{\sigma}_{h} \mathbf{G}_{8}=\mathbf{S}_{6}
\end{aligned}
$$

There are only a finite number of possible combinations of these basic operations which are of interest in the present work. These are diagrammed in table 3 and are known as crystallographic point groups, since each of the combinations constitutes a mathematical group, the elements of which leave the potential of a point unchanged. In interpreting table 3 , the starting point is the column headed $\mathbf{C}_{n}$. The groups $\mathbf{C}_{n}$ for the values of $n$ for which we are interested, consist of the operation $\mathbf{G}_{n}$ and its $n$ distinct powers, so a total of $n$ symmetry operations are represented in the group $\mathrm{C}_{n}$. This set of operations may be enlarged by adding the element I (moving along the diagonal to the left), as is indicated under the heading $\mathbf{C}_{n} \times \mathbf{I}$. This nota-
The designation $\mathrm{G}_{n}$ represents a $n$-fold axis of symmetry $(n=2,3,4,6)$, since the matrix is obtained from $\mathbb{G}_{\infty}$ by restricting $\phi$ to $2 \pi / n$. Each rotation by $2 \pi / n$ yields an equivalent point in the potential field and, including the original one, there are $n$ such altogether. 'The element $\mathbb{C}_{2}^{\prime}$ is a twofold axis of symmetry about the $y$-axis - it is characteristic of the collections of these symmetry operations to be emphasized here (the noncubic point groups) that there is at most one axis having more than twofold symmetry, and hence rotations about the $y$-axis can be restricted to those of order two. The element $\mathbb{I}$, the inversion, is noteworthy in the above as being the only one with a determinant -1 , all others being +1 . Viewed as an operation on a coordinate system, the inversion represents the transformation to left-handed rather than the usual right-handed system.

In addition to the symmetry operations given above, there are operations which may be represented as the product of the element $\mathbb{I}$ with those rotations given above. If we are considering a collection of these symmetry operations containing both I and the rotation, no fundamentally new information is obtained by considering the products, but there are certain collections of symmetry operations which include some of these product operations but not the factor operations separately. These product operations, together with their symbolic notation, are as follows:

$$
\begin{aligned}
& \mathbf{I C}_{2}^{\prime}=\left(\begin{array}{rrr}
1 & 0 & 0 \\
0 & -1 & 0 \\
0 & 0 & 1
\end{array}\right)=\sigma_{v} \\
& \mathbf{I C}_{4}^{3}=\left(\begin{array}{rrr}
0 & -1 & 0 \\
1 & 0 & 0 \\
0 & 0 & -1
\end{array}\right)=\sigma_{h} \mathbf{C}_{4}=\mathbf{S}_{4}
\end{aligned}
$$

tion represents the fact that $\mathbb{I}$ commutes with all powers of $\mathrm{C}_{n}$. The group $\mathrm{C}_{n}$ may also be enlarged by adding the element $\mathbb{C}_{2}^{\prime}$ (moving along the diagonal to the right). Here, the heading $\left\{\mathbf{G}_{n} \mathbf{C}_{2}^{\prime}\right\}$ represents that the various operations do not now necessarily commute, but that all possible combinations of them and their powers are included. Both of these enlarged groups have $2 n$ elements. To the latter set, I may now be added (moving farther to the right), obtaining $4 n$ operations. From these groups containing $\mathbb{1}$, in some cases groups of lower order may be obtained by suppressing half the elements, including the element I itself but not all products containing $\mathbb{I}$. For example, let us consider the group $\mathrm{C}_{2}$, which contains the elements $\mathbf{C}_{2}$ and $\mathbf{E}$. If we add $\mathbf{I}$, we 
TABLE 3. Structure of noncubic point groups

\begin{tabular}{l|c|c|c|c|c|c}
\hline Order & $\begin{array}{c}\text { Sub- } \\
\text { group }\end{array}$ & $\mathbf{C}_{n} \times \mathbf{I}$ & $\mathbf{C}_{n}$ & $\left\{\mathbf{C}_{n} \mathbf{C}_{2}^{\prime}\right\}$ & $\left\{\mathbf{C}_{n} \mathbf{C}_{2}\right\} \times \mathbf{I}$ & $\begin{array}{c}\text { Sub- } \\
\text { group }\end{array}$ \\
\hline
\end{tabular}

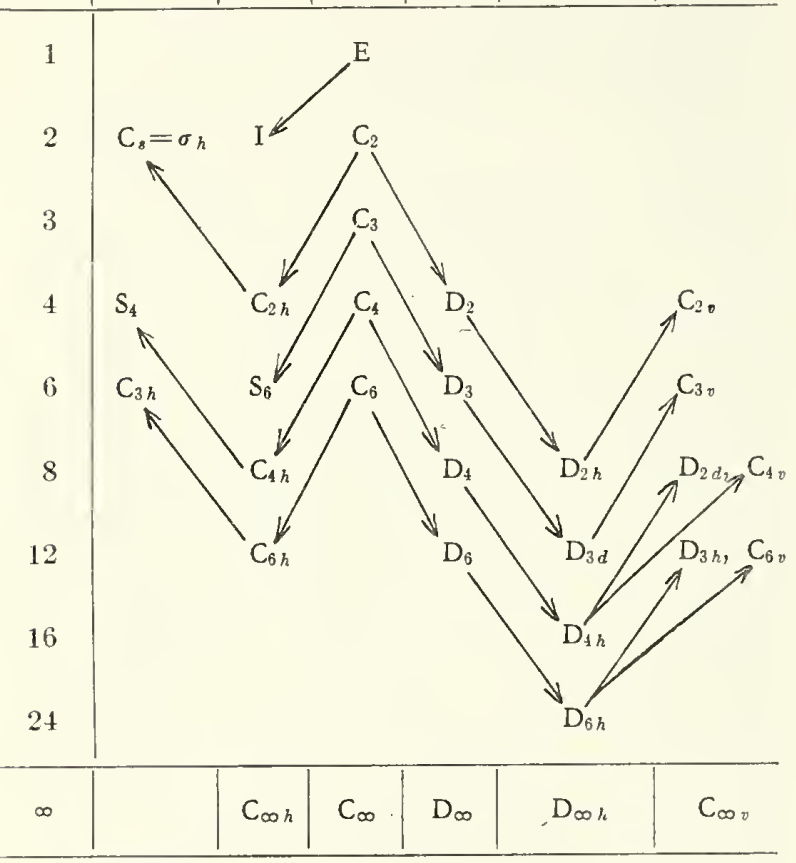

obtain $\mathrm{C}_{2 h}$, containing $\mathrm{C}_{2}, \mathrm{E}, \mathrm{C}_{2} \mathrm{I}$, and $\mathrm{I}$. From this we obtain $C_{s}$ by considering only $\mathbb{E}$ and $\mathrm{C}_{2} \mathrm{I}=\sigma_{h}$. Similarly, by adding $\mathrm{C}_{2}^{\prime}$ to $\mathrm{C}_{2}$, we obtain $\mathrm{D}_{2}$, which involves $\mathbb{E}, \mathbb{C}_{2}, \mathbb{C}_{2}^{\prime}$, and $\mathbb{C}_{2} \mathbb{C}_{2}^{\prime}$. By the further inclusion of $\mathbb{I}$, we obtain $\mathrm{D}_{2 h}$ with $\mathbb{E}, \mathbf{C}_{2}$, $\mathrm{C}_{2}^{\prime}, \mathrm{C}_{2} \mathrm{C}_{2}^{\prime}, \mathbb{I}, \mathbb{I} \mathrm{C}_{2}, \mathbb{I} \mathrm{C}_{2}^{\prime}$, and $\mathbf{I C}_{2} \mathrm{C}_{2}^{\prime}$. By suppressing $\mathbb{I}, \mathrm{IG}_{2}, \mathrm{C}_{2}^{\prime}$, and $\mathrm{C}_{2} \mathrm{C}_{2}^{\prime}$, the remaining four elements $\mathbf{E}, \mathrm{C}_{2}, \mathbb{I C}_{2}^{\prime}\left(=\sigma_{v}\right)$ and $\mathrm{C}_{2} \sigma_{v}$ constitute the group $\mathrm{C}_{2 v}$.

There are a few points which should be made clear in this connection. First, in the abstract mathematical sense, these groups are not all distinct. For example, the group $\mathrm{C}_{2}$ contains the elements $\mathbb{C}_{2}$ and $\mathbb{E}$, while the group I contains the elements $\mathbf{I}$ and $\mathbb{E}$, and they both have the same multiplication table. On the other hand, there is some degree of distinction which should be made between the $\mathbf{E}=\mathbf{C}_{2}^{2}$ (which we might call the identity in rotation space) and the $\mathbb{E}=\mathbb{I}^{2}$ (which we might call the identity in inversion space). Strictly speaking, the identity element of the groups such as $\mathrm{C}_{n} \times \mathbf{I}$ is the product of these two separate identity elements.

We shall now investigate, term by term, the symmetry possessed by the tesseral harmonics tabulated above. The overall symmetry of a given potential will then be at least that of those symmetry elements common to each term of the potential expansion. Conversely, if the symmetry is known from other considerations, we shall know what terms must be included to describe such a condition.

$\mathbf{C}_{\infty}$ : For this element to be present, the potential must be independent of $\phi$, a condition which is met only by terms with $M=0$ for all $N$.

$\mathrm{C}_{n}$ : For invariance here, $V(\phi)=V(\phi+2 \pi / n)$. Since the terms involve $\sin M \phi$ or $\cos M \phi$, it is clear that $n=M, M / 2, M / 3$, for integral values only, etc. We shall specify $n=M$, since the other cases are covered by the powers of $\mathbf{C}_{n}$ which are also present in any group.

$\mathrm{G}_{2}^{\prime}$ : A point with the coordinates $(r, \theta, \phi)$ under the transformation $\mathrm{C}_{2}^{\prime}$ becomes $(r, \pi-\theta, \pi-\phi)$. Since $\theta_{N}^{M}$ is a polynomial of degree $N-M$ in $\cos \theta$ and degree $M$ in $\sin \theta$, and $\cos (\pi-\theta)=-\cos \theta$, $\sin (\pi-\theta)=\sin \theta$, then $\theta_{N}^{M}$ changes as $(-1)^{N-M}$. However, $\sin M(\pi-\phi)=\sin M \pi \cos M \phi-\cos M_{\pi}$ $\sin M \phi=(-1)^{M+1}$ sin $M \phi$ and $\cos M(\pi-\phi)=$ $\cos M \pi \cos M \phi+\sin M \pi \sin M \phi=(-1)^{M} \cos M \phi$. Hence $C$ terms have this symmetry for $N$ even and $\mathrm{S}$ terms have it for $N$ odd.

I: Under this operation, the point $(x, y, z)$ becomes $(-x,-y,-z)$, and it is clear from inspection of table 2 that terms with even $N$ have this symmetry, while terms with odd $N$ do not.

$\sigma_{h}$ : This operation results, in rectangular coordinates, in the substitution of $-z$ for $z$, and, in spherical coordinates, in the substitution of $\pi-\theta$ for $\theta$. This is the same as the $\theta$ substitution in $\mathrm{G}_{2}^{\prime}$ and supplies a factor $(-1)^{N-M}$. Hence, $N$ and $M$ must both be even or both odd for this element.

$\boldsymbol{\sigma}_{v}:$ A vertical plane of symmetry exists at $\phi=\beta$ if $V(r, \theta, \beta+\alpha)=V(r, \theta, \beta-\alpha)$ for all values of $\alpha$. For $S$ terms, we have $\sin M(\beta+\alpha)=$ $\sin M \beta \cos M \alpha+\cos M \beta \sin M \alpha$ and $\sin M(\beta-\alpha)=$ $\sin M \beta \cos M \alpha-\cos M \beta \sin M \alpha$. This yields the requirement that $\cos M \beta \sin M \alpha=-\cos M \beta$ $\sin M \alpha=0$. For this to be independent of $\alpha$, $\cos M \beta=0, M \beta=\pi / 2,3 \pi / 2,5 \pi / 2$. . . and planes of symmetry exist at $\beta=\pi / 2 M, 3 \pi / 2 M$.

$(2 M-1) \pi / 2 M$. Similarly for $C$ terms, we obtain the requirement that $\sin M \beta \sin M \alpha=0, M \beta=0$, $\pi, 2 \pi, 3 \pi \ldots$ and planes of symmetry exist at $\beta=0, \pi / M, 2 \pi / M,(M-1) \pi / M$. We shall find it convenient to distinguish these sets of planes, describing those arising from $C$ terms as $\boldsymbol{\sigma}_{v}$ and those from $S$ terms as $\sigma_{d}$. Note that the set $\sigma_{n}$ for a given even $M$ includes both $\sigma_{v}$ and $\sigma_{d}$ for $M / 2$, and that any vertical plane is a plane of symmetry if $M=0$. Let us emphasize that the $x-z$ plane $(\beta=0)$ is a plane of symmetry for all $C$ terms, while the $y-z$ plane $\left(\beta=\frac{\pi}{2}\right)$ is a plane of symmetry for $C$ terms if $M$ is even, for $\mathrm{S}$ terms if $M$ is odd.

$\mathbf{S}_{4}$ : This operation transforms $(r, \theta, \phi)$ into $(r, \pi-\theta, \phi+(\pi / 2))$ and $\theta_{N}^{M}$ transforms as $(-1)^{N-M}$. S terms involve $\sin M(\phi+(\pi / 2))=\sin M \phi \cos M \pi / 2$ $+\cos M \phi \sin M \pi / 2$. $M$ odd yields a $\cos M \phi$ term which we reject, and $M$ even yields $(-1)^{M / 2} \sin M \phi$. Thus $S$ terms transform as $(-1)^{N-\frac{1}{2} M}$ for $M$ even. For $C$ terms, the $\theta$ contribution is the same as for $S$ terms and $\cos M(\phi+(\pi / 2))=\cos M \phi$ $\cos M \pi / 2-\sin M \phi \sin M \pi / 2$. As for $S$ terms, we reject $M$ odd and in $\phi$ obtain $(-1)^{M / 2} \cos M \phi$ for $M$ even. C terms then transform as $(-1)^{N-\frac{1}{2} M}$, 
just as $\mathbf{S}$ terms. The overall requirement for $\mathbf{S}_{4}$ is then that both $M$ and $N-\frac{1}{2} M$ must be even.

$\mathrm{S}_{6}$ : Since this element is defined as $\mathrm{C}_{3}^{2} \mathrm{I}$, it is necessary and sufficient that both $\mathrm{G}_{3}$ and $\mathrm{I}$ be present for this element to be present, and it need not be considered separately.

Based on the above analysis and discussion, the symmetry elements present in each of the tesseral harmonics of table 2 have been given in table 4 . An analysis of table 4 will indicate the potential coefficients (2.7) which must be included to represent a potential appropriate to the groups of table 3 , and the results of this analysis are given in table 5. Two points are worthy of note in the use of table 5 . In each case where, for a given $M$ and $N$ both $C$ and $S$ are shown, the first $S$ appearing is shown in parentheses. This represents the fact that, though both are consistent with the required symmetry, the $S$ term may be suppressed by a suitable rotation about the $z$-axis. This may be done, of course, for any onc $C, S$ pair, but only for one. All other indicated combinations of $C$ and $S$ must then be allowed, and this one $S$ omitted. Second, with the potential terms considered, it will be noted that the groups $D_{6}$ and $\mathrm{C}_{6 h}$ cannot be distinguished from the group $\mathrm{D}_{6 h}$ of higher symmetry, nor can $\mathrm{C}_{6}$ be distinguished from $\mathrm{C}_{60}$. Further analysis of the potential terms shows that the coefficients $S_{7}^{6}$, allowed in $D_{6}$, and $S_{8}^{6}$ in $C_{6 h}$, neither of which is allowed in $D_{6 h}$, are the lowest order terms separating these three groups. Similarly, $S_{7}^{6}$ in $C_{6}$ will separate it from $\mathrm{C}_{60}$, where this coefficient is forbidden. Finally, it will be noted that the groups $D_{\infty}, C_{\infty h}$, and $C_{\infty}$ have been omitted from the table. These symmetries do not arise from the electrostatic fields considered here.

\subsection{Preferred Coordinate Systems}

In considering the expansion of the potential (2.6) and the calculation of the coefficients (2.7), it is clear that the coefficients (2.7) will depend upon the choice of the coordinate systcm used.
On the other hand, it is also clcar that all expansions of the field (2.6) must be physically equivalent, and that a choice between two differing descriptions of the same field is merely a matter of choosing the simplest description. Following universal convention, the principal axis of symmetry in the preceding considcrations has been taken to be the $z$-axis, and in the calculation of the coefficients (2.7) this will have to be detcrmined by an inspection of the given configuration of charges, as will also the location of any $y$-axis $\left(\mathbf{C}_{2}^{\prime}\right)$. Any other choice of axis will, in general, yield a more complicatcd expansion, and the symmetry will be apparently (but not actually) lower.

It would be well to discuss further the point mentioned in the preceding subscction concerning the planes of symmetry. For a given $N$ and $M$, both $\mathrm{C}$ terms and $\mathrm{S}$ terms describe planes of symmetry, differing mercly in their oricntation with respect to the vertical coordinate plancs. Clearly, any linear combination of these two terms also represents a set of planes of symmetry, at some intermediatc angle. If only one $N$ and $M$ (other than $M=0$ ) is present, the linear combination will represent no mole information than will either one alone. The general preference arises from the fact that the actual calculation of matrix elements in section 5 will be done through the $\mathrm{Y}$ terms (2.8) rather than the $C$ and $S$ terms. It will be seen from (2.9) that the suppression of $S$ tcrms (as far as possible) will yield real coefficients for the matrix elements. A conventional preference for $C$ torms then implies the $x$ - $z$ plane as the preferred vertical planc of symmetry, and the $y$-axis for the operation $\mathbb{G}_{2}^{\prime}$, since $\mathbf{I C}_{2}^{\prime}$ is then a reflection in this preferred plane. Somctimes (e.g., $\mathrm{D}_{3 h}$ ), we shall admit $\mathrm{S}$ terms and keep $\mathbb{C}_{2}^{\prime}$ as the $y$-axis rather than adopt some other axis in the plane as $\mathrm{C}_{2}^{\prime}$.

Oncc an cxpansion has been obtained in a given coordinate system, that in any other coordinate system may in principlc be obtained from the fact that each of the tcrms (2.5) or (2.8) in the ncw coordinate system will be expressiblc as a sum of

TABLE 4. Symmetry elements in tesseral harmonics

\begin{tabular}{|c|c|c|c|c|c|c|c|}
\hline$M=$ & 0 & 1 & 2 & 3 & 4 & 5 & 6 \\
\hline $\begin{array}{r}N=1 \\
2 \\
C \quad 3 \\
\\
4 \\
5 \\
6\end{array}$ & $\begin{array}{l}\mathbf{C}_{\infty} \sigma_{\eta_{\nu}} \\
\mathbf{C}_{\infty} \mathbf{I} \mathbf{C}_{2}^{\prime} \sigma_{v} \mathbf{S}_{4} \sigma_{h} \\
\mathbf{C}_{\infty} \sigma_{\nu} \\
\mathbf{C}_{\infty} \mathbf{I} \mathbf{C}_{2}^{\prime} \sigma_{\nu} \mathbf{S}_{4} \sigma_{h} \\
\mathbf{C}_{\infty} \sigma_{\vartheta} \\
\mathbf{C}_{\infty} \mathbf{I} \mathbf{C}_{2}^{\prime} \sigma_{v} \mathbf{S}_{4} \sigma_{h}\end{array}$ & $\begin{array}{l}\boldsymbol{\sigma}_{h} \boldsymbol{\sigma}_{\eta} \\
\boldsymbol{\sigma}_{v} \mathbf{I} \mathbf{C}_{2}^{\prime} \\
\boldsymbol{\sigma}_{h} \boldsymbol{\sigma}_{v} \\
\boldsymbol{\sigma}_{v} \mathbf{I} \mathbf{C}_{2}^{\prime} \\
\boldsymbol{\sigma}_{h} \boldsymbol{\sigma}_{v} \\
\boldsymbol{\sigma}_{v} \mathbf{I} \mathbf{C}_{2}^{\prime}\end{array}$ & $\begin{array}{l}\mathrm{C}_{2} \mathbf{I} \mathbf{C}_{2}^{\prime} \sigma_{h} \sigma_{\nu} \\
\mathbf{C}_{2} \sigma_{v} \mathrm{~S}_{4} \\
\mathrm{C}_{2} \mathbf{I} \mathbf{C}_{2}^{\prime} \sigma_{h} \sigma_{0} \\
\mathrm{C}_{2} \sigma_{\nu} \mathbf{S}_{4} \\
\mathrm{C}_{2} \mathbf{I} \mathbf{C}_{2}^{\prime} \sigma_{h} \sigma_{v}\end{array}$ & $\begin{array}{l}\mathbf{C}_{3} \sigma_{h} \sigma_{v} \\
\mathbf{C}_{3} \mathbf{I} \mathbf{C}_{2}^{\prime} \sigma_{v} \mathbf{S}_{6} \\
\mathbf{C}_{3} \sigma_{h} \sigma_{v} \\
\mathbf{C}_{3} \mathbf{I} \mathbf{C}_{2}^{\prime} \sigma_{v} S_{6}\end{array}$ & $\begin{array}{l}\mathbf{C}_{4} I \mathbf{C}_{2}^{\prime} \sigma_{h} \sigma_{\nu} \mathrm{S}_{4} \\
\mathrm{C}_{4} \sigma_{\nu} \\
\mathrm{C}_{4} \mathbf{I} \mathbf{C}_{2}^{\prime} \sigma_{h} \sigma_{\nu} \mathrm{S}_{4}\end{array}$ & $\begin{array}{l}\mathbf{C}_{5} \sigma_{h} \sigma_{\vartheta} \\
\mathbf{C}_{5} \mathbf{I} \mathbf{C}_{2}^{\prime} \sigma_{\vartheta}\end{array}$ & $\mathbf{C}_{6} \mathbf{I} \mathbf{C}_{2}^{\prime} \sigma_{h} \sigma_{v} \mathbf{S}_{6}$ \\
\hline $\begin{array}{l}1 \\
2 \\
3 \\
4 \\
4 \\
5 \\
6\end{array}$ & & $\begin{array}{l}\sigma_{h} \sigma_{d} \mathbf{C}_{2}^{\prime} \\
\sigma_{d} \mathbf{I} \\
\sigma_{h} \sigma_{d} \mathbf{C}_{2}^{\prime} \\
\sigma_{d} \mathbf{I} \\
\sigma_{h} \sigma_{d} \mathbf{C}_{2}^{\prime} \\
\sigma_{d} \mathbf{I}\end{array}$ & $\begin{array}{l}\mathbf{C}_{2} \mathbf{I} \sigma_{h} \sigma_{d} \\
\mathbf{C}_{2} \mathbf{C}_{2}^{\prime} \sigma_{d} \mathbf{S}_{4} \\
\mathbf{C}_{2} I \sigma_{h} \sigma_{d} \\
\mathbf{C}_{2} \mathbf{C}_{2}^{\prime} \sigma_{d} \mathbf{S}_{4} \\
\mathbf{C}_{2} \mathbf{I} \sigma_{h} \sigma_{d}\end{array}$ & $\begin{array}{l}\mathrm{C}_{3} \mathrm{C}_{2}^{\prime} \sigma_{h} \sigma_{d} \\
\mathrm{C}_{3} \mathrm{I} \sigma_{d} \mathbf{S}_{6} \\
\mathrm{C}_{3} \mathbf{C}_{2}^{\prime} \sigma_{h} \sigma_{d} \\
\mathbf{C}_{3} \mathbf{I} \sigma_{d} S_{6}\end{array}$ & $\begin{array}{l}\mathrm{C}_{1} \mathbb{I} \sigma_{h} \sigma_{d} \mathbf{S}_{4} \\
\mathrm{C}_{4} \mathbf{C}_{2}^{\prime} \sigma_{d} \\
\mathbf{C}_{4} \mathbb{I} \sigma_{h} \sigma_{d} \mathbf{S}_{4}\end{array}$ & $\begin{array}{l}\mathrm{C}_{5} \mathbf{C}_{2}^{\prime} \sigma_{h} \sigma_{d} \\
\mathbf{C}_{5} \mathbf{I} \sigma_{d}\end{array}$ & $\mathbf{C}_{0} \mathbf{I} \sigma_{h} \sigma_{d} \mathbf{S}_{6}$ \\
\hline
\end{tabular}




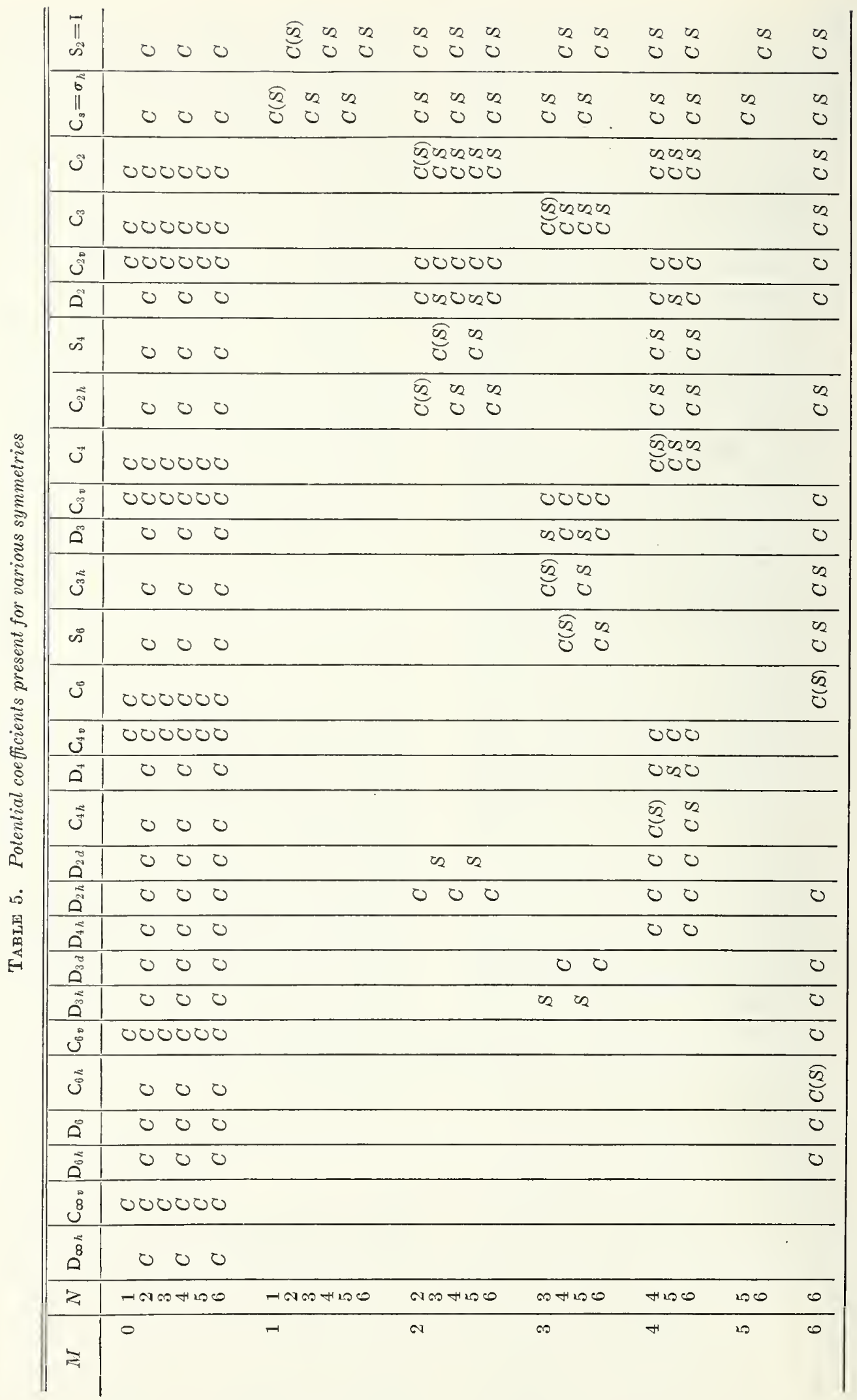


TABLE 6a. Transformation of tesseral harmonics

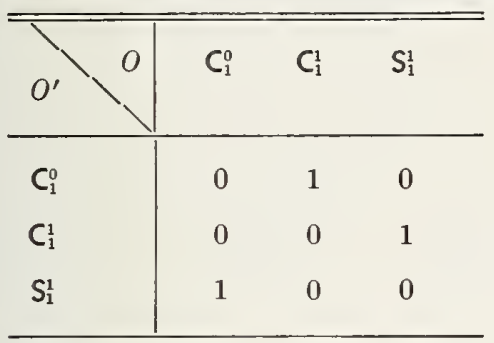

TABLE 6b. Transformation of tesseral harmonics

\begin{tabular}{l|rrrrr}
\hline \hline$O^{\prime} \backslash O$ & $\mathrm{C}_{2}^{0}$ & $\mathrm{C}_{2}^{1}$ & $\mathrm{C}_{2}^{2}$ & $\mathrm{~S}_{2}^{1}$ & $\mathrm{~S}_{2}^{3}$ \\
\hline $\mathrm{C}_{2}^{0}$ & $-\frac{1}{2}$ & 0 & $\frac{\sqrt{3}}{2}$ & 0 & 0 \\
$\mathrm{C}_{2}^{1}$ & 0 & 0 & 0 & 0 & 1 \\
$\mathrm{C}_{2}^{2}$ & $-\frac{\sqrt{3}}{2}$ & 0 & $-\frac{1}{2}$ & 0 & 0 \\
$\mathrm{~S}_{2}^{1}$ & 0 & 1 & 0 & 0 & 0 \\
$\mathrm{~S}_{2}^{2}$ & 0 & 0 & 0 & 1 & 0 \\
\hline
\end{tabular}

the $2 N+1$ terms of the same value of $N$ but of different $M$ in the old coordinate system. While general formulas have been derived for such relations (Wigner, 1931), a detailed study has been carried out for a second coordinate system $O^{\prime}$ obtained by a rotation of $O$ by $-120^{\circ}$ about the $(\mathbf{i}+\mathbf{j}+\mathbf{k})$ axis. 'This is equivalent to a cyclic permutation $x \rightarrow y \rightarrow z \rightarrow x$ of the variables; i.e., the functions $\mathbf{C}_{N}^{M}(y, z, x)$ and $\mathbf{S}_{N}^{M}(y, z, x)$ were evaluated in terms of $\mathbf{C}_{N}^{M I}(x, y, z)$ and $\mathbf{S}_{N}^{M I}(x, y, z)$. The results of this evaluation are presented as the matrices of table 6 . It should be noted that these matrices are orthogonal.

TABLE 6e. Transformation of tesseral harmonics

\begin{tabular}{l|rrrrrrr}
\hline & $C_{3}^{0}$ & $C_{3}^{1}$ & $C_{3}^{2}$ & $C_{3}^{3}$ & $S_{3}^{1}$ & $S_{3}^{2}$ & $S_{3}^{3}$ \\
\hline$C_{3}^{0}$ & 0 & $-\frac{\sqrt{6}}{4}$ & 0 & $\frac{\sqrt{10}}{4}$ & 0 & 0 & 0 \\
$C_{3}^{1}$ & 0 & 0 & 0 & 0 & $-\frac{1}{4}$ & 0 & $\frac{\sqrt{15}}{4}$ \\
$C_{3}^{2}$ & 0 & $-\frac{\sqrt{10}}{4}$ & 0 & $-\frac{\sqrt{6}}{4}$ & 0 & 0 & 0 \\
$C_{3}^{3}$ & 0 & 0 & 0 & 0 & $-\frac{\sqrt{15}}{4}$ & 0 & $-\frac{1}{4}$ \\
$S_{3}^{1}$ & $-\frac{\sqrt{6}}{4}$ & 0 & $\frac{\sqrt{10}}{4}$ & 0 & 0 & 0 & 0 \\
$\mathrm{~S}_{3}^{2}$ & 0 & 0 & 0 & 0 & 0 & 1 & 0 \\
$\mathrm{~S}_{3}^{3}$ & $-\frac{\sqrt{10}}{4}$ & 0 & $-\frac{\sqrt{6}}{4}$ & 0 & 0 & 0 & 0 \\
\hline
\end{tabular}

TABLE 6d. Transformation of tesseral harmonics

\begin{tabular}{|c|c|c|c|c|c|c|c|c|c|}
\hline$O^{\prime}$ & $\mathrm{C}_{4}^{0}$ & $C_{4}^{1}$ & $\mathrm{C}_{\mathbf{4}}^{2}$ & $C_{4}^{3}$ & $C_{4}^{4}$ & $S_{4}^{1}$ & $S_{4}^{2}$ & $\mathrm{~S}_{1}^{3}$ & $S_{4}^{4}$ \\
\hline $\mathrm{C}_{4}^{0}$ & $\frac{3}{8}$ & 0 & $-\frac{\sqrt{5}}{4}$ & 0 & $\frac{\sqrt{35}}{8}$ & 0 & 0 & 0 & 0 \\
\hline$C_{4}^{1}$ & 0 & 0 & 0 & 0 & 0 & 0 & $-\frac{\sqrt{2}}{4}$ & 0 & $\frac{\sqrt{14}}{4}$ \\
\hline$C_{4}^{2}$ & $\sqrt{\frac{5}{4}}$ & 0 & $-\frac{1}{2}$ & 0 & $-\sqrt{\overline{7}}$ & 0 & 0 & 0 & 0 \\
\hline$C_{4}^{3}$ & 0 & 0 & 0 & 0 & 0 & 0 & $-\frac{\sqrt{14}}{4}$ & 0 & $-\frac{\sqrt{2}}{4}$ \\
\hline$C_{4}^{4}$ & $\frac{\sqrt{35}}{8}$ & 0 & $\frac{\sqrt{7}}{4}$ & 0 & $\frac{1}{8}$ & 0 & 0 & 0 & 0 \\
\hline $\mathrm{S}_{4}^{1}$ & 0 & $-\frac{3}{4}$ & 0 & $\frac{\sqrt{7}}{4}$ & 0 & 0 & 0 & 0 & 0 \\
\hline $\mathrm{S}_{1}^{2}$ & 0 & 0 & 0 & 0 & 0 & $-\frac{\sqrt{2}}{4}$ & 0 & $\frac{\sqrt{14}}{4}$ & 0 \\
\hline $\mathrm{S}_{4}^{3}$ & 0 & $-\frac{\sqrt{7}}{4}$ & 0 & $-\frac{3}{4}$ & 0 & 0 & 0 & 0 & 0 \\
\hline$S_{4}^{4}$ & 0 & 0 & 0 & 0 & 0 & $-\frac{\sqrt{14}}{4}$ & 0 & $-\sqrt{\frac{2}{4}}$ & 0 \\
\hline
\end{tabular}


TABLE 6e. Transformation of tesseral harmonics

\begin{tabular}{|c|c|c|c|c|c|c|c|c|c|c|c|}
\hline$O$ & $\mathrm{C}_{5}^{0}$ & $C_{5}^{1}$ & $C_{5}^{2}$ & $C_{5}^{3}$ & $C_{5}^{4}$ & $C_{5}^{5}$ & $S_{5}^{2}$ & $\mathrm{~S}_{5}^{2}$ & $S_{5}^{3}$ & $S_{5}^{4}$ & $S_{5}^{5}$ \\
\hline $\mathrm{C}_{5}^{0}$ & 0 & $\frac{\sqrt{15}}{8}$ & 0 & $-\frac{\sqrt{70}}{16}$ & 0 & $\frac{3 \sqrt{14}}{16}$ & 0 & 0 & 0 & 0 & 0 \\
\hline $\mathrm{C}_{6}^{1}$ & 0 & 0 & 0 & 0 & 0 & 0 & $\frac{1}{8}$ & 0 & $-\frac{\sqrt{42}}{16}$ & 0 & $\frac{\sqrt{210}}{16}$ \\
\hline$C_{5}^{2}$ & 0 & $\frac{\sqrt{7}}{4}$ & 0 & $-\frac{\sqrt{6}}{8}$ & 0 & $-\frac{\sqrt{30}}{8}$ & 0 & 0 & 0 & 0 & 0 \\
\hline$C_{6}^{3}$ & 0 & 0 & 0 & 0 & 0 & 0 & $\frac{\sqrt{42}}{16}$ & 0 & $-\frac{13}{16}$ & 0 & $-\frac{3 \sqrt{5}}{16}$ \\
\hline$C_{5}^{4}$ & 0 & $\frac{\sqrt{21}}{8}$ & 0 & $\frac{9 \sqrt{2}}{16}$ & 0 & $\frac{\sqrt{10}}{16}$ & 0 & 0 & 0 & 0 & 0 \\
\hline$C_{5}^{6}$ & 0 & 0 & 0 & 0 & 0 & 0 & $\frac{\sqrt{210}}{16}$ & 0 & $\frac{3 \sqrt{5}}{16}$ & 0 & $\frac{1}{16}$ \\
\hline $\mathbf{S}_{5}^{1}$ & $\frac{\sqrt{15}}{8}$ & 0 & $-\frac{\sqrt{7}}{4}$ & 0 & $\frac{\sqrt{21}}{8}$ & 0 & 0 & 0 & 0 & 0 & 0 \\
\hline $\mathrm{S}_{5}^{2}$ & 0 & 0 & 0 & 0 & 0 & 0 & 0 & $-\frac{1}{2}$ & 0 & $\sqrt{\frac{3}{2}}$ & 0 \\
\hline $\mathrm{S}_{5}^{3}$ & $\frac{\sqrt{70}}{16}$ & 0 & $-\frac{\sqrt{6}}{8}$ & 0 & $-\frac{9 \sqrt{2}}{16}$ & 0 & 0 & 0 & 0 & 0 & 0 \\
\hline $\mathbf{S}_{5}^{4}$ & 0 & 0 & 0 & 0 & 0 & 0 & 0 & $-\frac{\sqrt{3}}{2}$ & 0 & $-\frac{1}{2}$ & 0 \\
\hline $\mathrm{S}_{5}^{5}$ & $\frac{3 \sqrt{14}}{16}$ & 0 & $\frac{\sqrt{30}}{8}$ & 0 & $\frac{\sqrt{10}}{16}$ & 0 & 0 & 0 & 0 & 0 & 0 \\
\hline
\end{tabular}

\subsection{Cubic Point Groups}

In addition to the 27 noncubic point groups of table 3 , there are 5 point groups known as the cubic groups. While these are not known to arise in natural rare earth crystals, they are important in the consideration of crystals of other types; e.g., the iron group, and some data on rare earth ions have been obtained by the inclusion of the ion in a foreign lattice of cubic symmetry (Low, 1958). We shall therefore complete the discussion by considering these groups.

The five groups are assigned the symbols $\mathrm{O}$, $\mathrm{O}_{h}=(\mathrm{O} \times \mathrm{I}), \mathrm{T}, \mathrm{T}_{h}=(\mathrm{T} \times \mathrm{I})$, and $\mathrm{T}_{d}$, but only four distinct abstract groups are involved since $\mathrm{T}_{d}$ and $O$ are isomorphic. The group $O$ has 24 elements and represents the purely rotational symmetry of the cube (or octahedron), while $\mathrm{O}_{h}, 48$ elements, represents the full symmetry of the cube. $T$ is of order 12 and represents the pure rotational symmetry of the tetrahedron, while $\mathrm{T}_{d}$, a subgroup of $\mathrm{O}_{h}$ with 24 elements, represents the full symmetry of the regular tetrahedron. $\mathrm{T}_{h}$ is also of order 24.

These groups represent a higher degree of symmetry than our previous considerations have suggested. In particular, $O$ includes fourfold axes along $x, y$, and $z$, threefold axes along the principal diagonal of each octant, and twofold axes along the diagonals of the coordinate planes. $T$ has 4 threefold axes, of which at most one can be along a coordinate axis, but has also three mutually perpendicular twofold axes. Rotations of these groups will be generally designated $\mathbf{C}_{n}$ for rotations $\frac{2 \pi}{n}$, and they may be further distinguished by primes. However, the distinctive conventions of section 2.2 which are appropriate for $\mathrm{D}_{\infty h}$ and its subgroups, cannot be adhered to for these high symmetries. These symmetries cannot arise from considering the symmetry elements common to a sum of terms as in the previous cases, but depend upon certain fixed ratios in the coefficients. In other words, these may be viewed as higher symmetries arising from "accidental" values of the coefficients in a case of lower symmetry.

As a particular example, let us consider the group $\mathrm{D}_{4}$, representing the rotational symmetry of a rectangular prism, with potential coefficients (table 5) $C_{2}^{0}, C_{4}^{0}, C_{6}^{0}, C_{4}^{4}, S_{5}^{4}$, and $C_{8}^{4}$. The $x$ and $y$ axes are equivalent, but the long dimension, the $z$ axis, will generally be different. If the prism actually becomes a cube, the $x, y$, and $z$ axes will all be equivalent. Hence we expect that any combination of these terms which is invariant under a substitution $x \rightarrow y \rightarrow z \rightarrow x$ will represent a higher symmetry, in this case $O$. This is just the substitution considered in table 6. We see, for 


\begin{tabular}{|c|c|c|c|c|c|c|c|c|c|c|c|c|c|}
\hline $0^{\prime}$ & $C_{6}^{0}$ & $C_{6}^{1}$ & $C_{6}^{2}$ & $\mathbf{C}_{6}^{3}$ & $\mathrm{C}_{6}^{4}$ & $C_{6}^{5}$ & $\mathrm{C}_{6}^{6}$ & $\mathrm{~S}_{6}^{1}$ & $\mathbf{S}_{6}^{2}$ & $\mathbf{S}_{6}^{3}$ & $\mathrm{~S}_{6}^{4}$ & $\mathrm{~S}_{6}^{5}$ & $\mathbf{S}_{6}^{0}$ \\
\hline $\mathrm{C}_{6}^{0}$ & $-\frac{5}{16}$ & 0 & $\frac{\sqrt{210}}{32}$ & 0 & $-\frac{3 \sqrt{7}}{16}$ & 0 & $\frac{\sqrt{462}}{32}$ & 0 & 0 & 0 & 0 & 0 & 0 \\
\hline$C_{6}^{1}$ & 0 & 0 & 0 & 0 & 0 & 0 & 0 & 0 & $\frac{\sqrt{10}}{16}$ & 0 & $-\frac{\sqrt{3}}{4}$ & 0 & $\frac{3 \sqrt{22}}{16}$ \\
\hline $\mathrm{C}_{\tilde{\delta}}^{2}$ & $-\frac{\sqrt{210}}{32}$ & 0 & $\frac{17}{32}$ & 0 & $-\frac{\sqrt{30}}{32}$ & 0 & $-\frac{3 \sqrt{55}}{32}$ & 0 & 0 & 0 & 0 & 0 & 0 \\
\hline $\mathrm{C}_{6}^{3}$ & 0 & 0 & 0 & 0 & 0 & 0 & 0 & 0 & $\frac{9}{16}$ & 0 & $-\frac{\sqrt{30}}{8}$ & 0 & $-\frac{\sqrt{55}}{16}$ \\
\hline$C_{6}^{4}$ & $-\frac{3 \sqrt{7}}{16}$ & 0 & $\frac{\sqrt{30}}{32}$ & 0 & $\frac{13}{16}$ & 0 & $\frac{\sqrt{66}}{32}$ & 0 & 0 & 0 & 0 & 0 & 0 \\
\hline$C_{6}^{5}$ & 0 & 0 & 0 & 0 & 0 & 0 & 0 & 0 & $\frac{\sqrt{165}}{16}$ & 0 & $\sqrt{\frac{22}{8}}$ & 0 & $\frac{\sqrt{3}}{16}$ \\
\hline $\mathrm{C}_{6}^{6}$ & $-\frac{\sqrt{462}}{32}$ & 0 & $-\frac{3 \sqrt{55}}{32}$ & 0 & $-\frac{\sqrt{66}}{32}$ & 0 & $-\frac{1}{32}$ & 0 & 0 & 0 & 0 & 0 & 0 \\
\hline$S_{6}^{1}$ & 0 & $\frac{5}{8}$ & 0 & $-\frac{3 \sqrt{10}}{16}$ & 0 & $\frac{\sqrt{66}}{16}$ & 0 & 0 & 0 & 0 & 0 & 0 & 0 \\
\hline$S_{6}^{2}$ & 0 & 0 & 0 & 0 & 0 & 0 & 0 & $\frac{\sqrt{10}}{16}$ & 0 & $-\frac{9}{16}$ & 0 & $\frac{\sqrt{165}}{16}$ & 0 \\
\hline$S_{6}^{3}$ & 0 & $\frac{3 \sqrt{10}}{16}$ & 0 & $-\frac{1}{16}$ & 0 & $-\frac{\sqrt{165}}{16}$ & 0 & 0 & 0 & 0 & 0 & 0 & 0 \\
\hline$S_{8}^{4}$ & 0 & 0 & 0 & 0 & 0 & 0 & 0 & $\frac{\sqrt{3}}{4}$ & 0 & $-\frac{\sqrt{30}}{8}$ & 0 & $-\frac{\sqrt{22}}{8}$ & 0 \\
\hline$S_{0}^{5}$ & 0 & $\frac{\sqrt{66}}{16}$ & 0 & $\frac{\sqrt{165}}{16}$ & 0 & $\frac{5}{16}$ & 0 & 0 & 0 & 0 & 0 & 0 & 0 \\
\hline$S_{0}^{\infty}$ & 0 & 0 & 0 & 0 & 0 & 0 & 0 & $\frac{3 \sqrt{22}}{16}$ & 0 & $\frac{\sqrt{55}}{16}$ & 0 & $\frac{\sqrt{3}}{16}$ & 0 \\
\hline
\end{tabular}

example, that $\mathrm{C}_{2}^{0}$ becomes a combination of $\mathrm{C}_{2}^{0}$ and $\mathrm{C}_{2}^{2}$. Since $\mathrm{C}_{2}^{2}$ is not allowed in $\mathrm{D}_{4}$, we suppress the second degree terms and proceed to those of the fourth degree. Although both $C_{4}^{0}$ and $C_{4}^{4}$ yield $\mathrm{C}_{4}^{2}$, if the coefficients are in the ratio $C_{4}^{0}: C_{4}^{4}=$ $\sqrt{7}: \sqrt{5}$, the $C_{4}^{2}$ will cancel and the combination $\mathrm{C}_{4}^{0}+\sqrt{\frac{5}{7}} \mathrm{C}_{4}^{4}$ will be invariant under the operation of table 6 . Similarly, if $C_{6}^{0}: C_{6}^{4}=-1: \sqrt{7}, C_{6}^{2}$ and $\mathrm{C}_{6}^{6}$ arising from the transformation will vanish and $C_{6}^{0}-\sqrt{7} C_{6}^{4}$ will also be invariant. $S_{5}^{4}$ will admix $S_{5}^{2}$, forbidden in $\mathrm{D}_{4}$, so $S_{5}^{4}$ must also vanish. We conclude then that under these conditions, we have but two coefficients, $C_{4}^{0}$ and $C_{6}^{0}$ left to fix the strength of the field, and the symmetry is $\mathrm{O}$ rather than $\mathrm{D}_{4}$. In fact, all of the terms aliowed also contain I as a symmetry element, and the symmetry is even higher, i.e., $\mathrm{O}_{h}$. The distinction between $\mathrm{O}$ and $\mathrm{O}_{h}$ will arise from potential terms of higher order.

On the other hand, the cube may be considered as standing on a corner with a threefold axis along the $z$ axis, arising as a special case of $D_{3}$ symmetry. The allowed coefficients will be generally $C_{2}^{0} ; S_{3}^{3}$; $C_{4}^{0}, C_{4}^{3} ; S_{5}^{3} ; C_{6}^{0}, C_{6}^{3}$, and $C_{6}^{6}$. Since we do not have data corresponding to table 6 to apply our previous method, we shall set up a charge configuration having the desired symmetry and orientation, evaluate the coefficients (2.7) and determine the necessary relations among them. This has been done in detail as an example in section 6-we report merely the result at this point. The coefficients $C_{2}^{0}, S_{3}^{3}$, and $S_{5}^{3}$ must vanish, while the ratios among the nonvanishing coefficients are $C_{4}^{0}: C_{4}^{3}=-7: 2 \sqrt{35}$ and $C_{6}^{0}: C_{6}^{3}: C_{6}^{6}=24: 2 \sqrt{105}: \sqrt{462}$. In this case as well, $C_{4}^{0}$ and $C_{6}^{0}$ are the only adjustable parameters to fix the strength of the field.

The group T may likewise be considered either as a special case of $D_{2}$, where the $x, y$, and $z$ axes all become equivalent, or as a special case of $\mathrm{C}_{3}$ with the principal threefold axis of the figure vertical. In the former case, the allowed coefficients are: $C_{2}^{0} ; C_{2}^{2} ; S_{3}^{2} ; C_{4}^{0}, C_{4}^{2}, C_{4}^{4} ; S_{5}^{2}, S_{5}^{4}$; and $C_{6}^{0}$, $C_{6}^{2}, C_{6}^{4}$, and $C_{6}^{6}$. A consideration of these terms and table 6 shows that the following conditions 
must be met:

$$
\begin{array}{r}
C_{2}^{0}=C_{2}^{2}=0 \quad S_{3}^{2} \text { is arbitrary } \quad C_{4}^{2}=0 \quad S_{5}^{2}=S_{5}^{4}=0 \\
C_{4}^{0}: C_{4}^{4}=\sqrt{7} \cdot \sqrt{5} \quad C_{6}^{0}: C_{6}^{4}=-1: \sqrt{7} \\
\quad \text { and } C_{6}^{2}: C_{6}^{6}=-11: \sqrt{55} .
\end{array}
$$

The constants $S_{3}^{2}, C_{4}^{0}, C_{6}^{0}$ and $C_{6}^{2}$ can be considered the variable coefficients describing the strength of the field. The symmetry becomes $\mathrm{T}_{h}$ if the only one of these terms lacking the element I, $S_{3}^{2}$, is suppressed. If we start from the case of $D_{2 d}$ symmetry instead of merely $\mathrm{D}_{2}$, we obtain $\mathrm{T}_{a}$ symmetry but must suppress the coefficient $C_{6}^{2}$, leaving $S_{3}^{2}, C_{4}^{0}$, and $C_{6}^{0}$.
In evaluating the coefficients for the threefold axis vertical, we have again had recourse to the establishment of a specific configuration, namely; that of the regular tetrahedron itself. This will yield terms for $T_{d}$ rather than $T$ or $T_{h}$. Configurations for these latter symmetries are considerably more complicated, and have not been evaluated for this orientation. The nonvanishing coefficients must meet the following conditions:

$$
\begin{aligned}
C_{3}^{0}: C_{3}^{3}=\sqrt{5}: 2 \quad & C_{4}^{0}: C_{4}^{3}=-\sqrt{35}: 5 \quad \text { and } \\
& C_{6}^{0}: C_{6}^{3}: C_{6}^{6}=4 \sqrt{2310}: 77 \sqrt{5}: 35 \sqrt{22} .
\end{aligned}
$$

\section{Groups, Characters, and Representations}

\subsection{Representations and Quantum Mechanics}

Let us consider a Hamiltonian $\mathbb{H}$, and $\psi_{i}(i=$ $1,2, \ldots n$ ) an $n$-fold degenerate solution of the Schroedinger equation $\boldsymbol{H} \psi_{i}=E \psi_{i}$. Let us further consider a group of transformations (generally rotations or the inversion) which may be applied to this equation. If an element of the group is $\mathbf{P}$, with inverse $\mathbf{P}^{-1}$, then $\mathbf{P H} \psi_{i}=\mathbf{P} E \psi_{i}$, which may be rewritten as

$$
\left(\mathbf{P H P} \mathbf{P}^{-1}\right)\left(\mathbf{P} \psi_{i}\right)=E\left(\mathbf{P} \psi_{i}\right) .
$$

This we interpret as yielding a transformed Hamiltonian $\left(\mathrm{PHP}^{-1}\right)$ and a transformed solution $\mathbb{P} \psi_{i}$ of the Schroedinger equation. Let us now assume that the Hamiltonian is invariant under the group of operations; i.e., that $\mathbb{P H P}^{-1}=\mathbf{H}$ or $\mathbf{P H}=\mathbf{H P}$, and that $\mathbf{Q}$ is another operation of the group. Then (3.1) becomes $\mathbb{H}\left(\mathbb{P} \psi_{i}\right)=E\left(\mathbb{P} \psi_{i}\right)$, and $\mathbf{P} \psi_{i}$ must be a solution of the original wave equation with the same energy; i.e., it must be a linear combination of the original wave functions

$$
\mathrm{P} \psi_{i}=\sum_{j} P_{i j} \psi_{j}
$$

Similarly $\mathrm{Q} \psi_{i}=\sum_{j} Q_{i j} \psi_{j}$, and $(\mathrm{QP}) \psi_{i}=\sum_{j}(Q P)_{i j} \psi_{j}$. Thus the effect of each operation of the group may be expressed as a $n \times n$ unitary matrix. The importance of the concept becomes most evident when we consider the product QP as the operation $\mathbf{P}$ followed by the operation $\mathbf{Q}$ :

$$
\begin{aligned}
\mathbf{Q}\left(\mathbb{P} \psi_{i}\right)=\mathbf{Q}\left(\sum_{j} P_{i j} \psi_{j}\right)=\sum_{k} Q_{i k} \sum_{j} P_{k j} \psi_{j} \\
=\sum_{j} \sum_{k} Q_{i k} P_{k j} \psi_{j} .
\end{aligned}
$$

Thus, the matrix for (QP) is the product of the matrix for $\mathbf{P}$ by that for $\mathbf{Q}$. These unitary matrices are called a "representation of the group" because any relation between elements of the group is also a relation between the matrices corresponding to the group elements.
Clearly, the precise form of the matrices depends on the particular choice of original wave functions, and any other set, related to the original set by a unitary transformation may be selected and will yield a transformed representation of the group. If it is possible to find a transformation such that the group operations cause only $m$ of the $n$ wave functions to transform among themselves, and the remaining $n-m$ functions among themselves, the representation is said to be (fully) reducible, otherwise irreducible. Following Melvin (1956), we shall for brevity speak of an irreducible representation as a "rep". If the representation is a rep, the degeneracy is said to be essential, since the $n$ wave functions may be transformed into each other purely by symmetry operations. If the representation can be reduced into two or more reps, the degeneracy is essential between wave functions belong to the same rep, but "accidental" when wave functions belonging to different reps are concerned, since wave functions belonging to different reps are not related to each other purely by symmetry operations. Accidental degeneracy is either due to a purely fortuitous consequence of the numerical parameters of the system under consideration, or to the presence of additional symmetry not previously considered (sometimes called "excess degeneracy"). An example of this latter will be considered in connection with Kramers' theorem (section 4.2), which states that all levels of an atomic system with an odd number of electrons will be at least twofold degenerate under the influence of external fields of purely electrostatic character. In this case, it will be seen that wave functions belonging to different reps of the rotation-reflection group may actually be related by symmetry with respect to time reversal.

\subsection{Rotations in Three Dimensions}

One of the transformations in which we shall be particularly interested is that of an arbitrary rotation of the coordinate axes in three dimensions, so we shall now examine this in some detail. Let us 
consider a fixed $(\boldsymbol{x}, \boldsymbol{y}, \boldsymbol{z})$ reference frame, and a morable frame $(\xi, \eta, \xi)$, the origin of the latter being fixed at the origin of the $(\boldsymbol{x}, \boldsymbol{y}, \boldsymbol{z})$ frame. We assume initially that the $\xi$, $\eta$, and $\zeta$ axes coincide with the $x, y$, and $z$ axes, respectively. Any rotation will move the $(\xi, \eta, \xi)$ frame to some new orientation which is uniquely determined by the rotation performed. We are interested in the rarious ways of specifving the orientation of the $(\boldsymbol{\xi}, \boldsymbol{\eta}, \boldsymbol{\zeta})$ frame and its relation to the rotation incolved.

It is clear that there will be three independent quantities to be specified, two to fix the direction of the axis of rotation and one to fix the angle of rotation about this axis. Consider a vector $\mathbf{r}$ fixed in the $(\xi, \eta, \xi)$ frame rotated through an angle $\omega$ about an axis fixed in $(\boldsymbol{x}, \boldsymbol{y}, \boldsymbol{z})$ containing the unit vector $\mathbf{u}$ with direction cosines $(\alpha, \beta, \gamma)$. Observe that $\mathbf{r}$ may be resolved into a component parallel to $\mathbf{u},(\mathbf{r} \cdot \mathbf{u}) \mathbf{u}$, and a component perpendicular to $\mathbf{u},(\mathbf{r}-(\mathbf{r} \cdot \mathbf{u}) \mathbf{u})$, and that the vector $(\mathbf{u} \times \mathbf{r})$ is perpendicular to both and has the same magnitude $r$ sin $(\mathbf{u}, \mathbf{r})$ as the latter.

The component of $\mathbf{r}$ along $\mathbf{u},(\mathbf{r} \cdot \mathbf{u}) \mathbf{u}$ will be unchanged, while the perpendicular component $\mathbf{r}-(\mathbf{r} \cdot \mathbf{u}) \mathbf{u}$ will be rotated through the angle $\omega$ into $(\mathbf{r}-(\mathbf{r} \cdot \mathbf{u}) \mathbf{u}) \cos \omega+(\mathbf{u} \times \mathbf{r}) \sin \omega$. Thus, $\mathbf{r}^{\prime}=\mathbf{r} \cos \omega+(1-\cos \omega)(\mathbf{r} \cdot \mathbf{u}) \mathbf{u}+(\mathbf{u} \times \mathbf{r}) \sin \omega$.

This may be written as the matrix equation $\mathbf{r}^{\prime}=\mathbf{D}_{1}(\mathbf{u}, \omega) \mathbf{r}$, where

$$
\mathbf{D}_{1}(\mathbf{u}, \omega)=\left(\begin{array}{lll}
\cos \omega+(1-\cos \omega) \alpha^{2} & (1-\cos \omega) \alpha \beta-\gamma \sin \omega & (1-\cos \omega) \alpha \gamma+\beta \sin \omega \\
(1-\cos \omega) \alpha \beta+\gamma \sin \omega & \cos \omega+(1-\cos \omega) \beta^{2} & (1-\cos \omega) \beta \gamma-\alpha \sin \omega \\
(1-\cos \omega) \alpha \gamma-\beta \sin \omega & (1-\cos \omega) \gamma \beta+\alpha \sin \omega & \cos \omega+(1-\cos \omega) \gamma^{2}
\end{array}\right)
$$

and $\mathbf{r}, \mathbf{r}^{\prime}$ are the column vectors $(\xi, \eta, \zeta)$ or $(x, y, z)$, and $\left(x^{\prime}, y^{\prime}, z^{\prime}\right)$, respectively.

Since $\mathbf{r}$ is fixed in the $(\xi, \eta, \zeta)$ frame, its components in that frame are the same before and after the rotation; they are the same as its coordinates $(x, y, z)$ before the rotation in the fixed frame.

The direction of the $(\xi, \boldsymbol{\eta}, \boldsymbol{\xi})$ frame may be determined then by applying this relation to the vectors $(1,0,0),(0,1,0)$, and $(0,0,1)$ in turn.

The set of all three dimensional rotations constitutes a group which we shall designate as $\Re_{3}$. The set of real matrices $D_{1}(\mathbf{u}, \omega)$ is orthogonal, hence unitary, and meets the requirements of section 3.1 for a representation of the group. (Note that $\mathrm{D}_{1}$ is a particular element of $D_{1}$.) It is important that the group of matrices $D_{1}$ be not confused with the group of abstract operations $\Re_{3}$, but this requires a clear understanding of the difference between a group and any particular representation of the group. In elementary work, a three-dimensional rotation is invariably thought of as an operation on a vector. This is adequate in these elementary cases, since this leads, as we have seen, to a particularly simple faithful representation (a unique matrix for each operation) of the group. However, we have already mentioned in section 2.3 that an element of $\Re_{3}$ will induce not only the linear substitution $\mathrm{D}_{1}$ among the three components of a vector, but also a linear substitution $\mathrm{D}_{N}$ among the $2 N+1$ components of the tesseral harmonics of degree $N$ (table 2). All these substitutions are equally well representations of $\Re_{3}$, and they are faithful for $N>0$. Geometrically, a vector may be resolved into its components, which are the tesseral harmonics of degree 1, which lie along the three coordinate axes, so that a linear substitution among the components of the tesseral harmonics of degree 1 necessarily implies a corresponding linear substitution among the components of a vector, which we interpret as a rotation of the vector. Likewise, any linear combination of the $2 N+1$ tesseral harmonics of degree $N$ (or any quantity having similar transformation properties) may be viewed as a vector in a space of $2 N+1$ dimensions, with the $2 N+1$ tesseral harmonics serving as the unit vectors. An element of $\Re_{3}$ may also be represented by a rotation matrix in this $2 N+1$ dimensional space. Our choice of properly normalized tesseral harmonics ensures that the resulting matrices are orthogonal. In terms of this concept, the matrices of table 6 are the matrices $\mathrm{D}_{N}$ for $N=1,2,3,4,5,6$ and

$$
\mathbf{u}=\frac{1}{\sqrt{3}}(\mathbf{i}+\mathbf{j}+\mathbf{k}), \omega=\frac{2 \pi}{3} .
$$

A general rotation may also be described by a point along the direction of $\mathbf{u}$ and at a distance $\omega$ from the origin. All rotations are thus represented as points within or on the surface of a sphere of radius $\pi$. More precisely, the points represent the results of the rotation, while the details of the rotation are fixed by prescribing a path from the origin (representing the original orientation) to this particular end point. It is important to notice that all points of the closed sphere represent distinct orientations, save those on the surface where diametrically opposed pairs of points represent the same orientation, being attained by a rotation $\pi$ about oppositely directed axes. Consequently, there are two essentially different types of paths by which one can go from the origin to another point within the sphere. Thus, if it is desired to reach the point $\left(0, \frac{\pi}{2}, 0\right)$ in the $(\mathbf{u} \omega)$ space, this could be accomplished by a single rotation $\frac{\pi}{2}$ about 
the $y$-axis, and the resulting path is a straight line from $(0,0,0)$ to $\left(0, \frac{\pi}{2}, 0\right)$. On the other hand, a rotation by $\pi$ about the $-y$ axis will be represented by a straight line from $(0,0,0)$ to $(0,-\pi$, $0)$. Since the latter point is equivalent to $(0, \pi, 0)$, a further rotation $\frac{\pi}{2}$ will result in a path from $(0, \pi, 0)$ to $\left(0, \frac{\pi}{2}, 0\right)$. We have thus reached $\left(0, \frac{\pi}{2}, 0\right)$ by two different paths. These are essentially different since there is no continuous deformation of the paths, keeping the end points fixed, which will cause them to coincide. Paths which can thus be made to coincide are said to be of the same homotopy class, otherwise they are of a different homotopy class. There are only two homotopy classes for rotation in three dimensions. We shall call them $P_{0}$ (for paths involving no points on the surface of the sphere, or an even number of pairs of such points) and $P_{1}$ (paths involving an odd number of pairs of points on the surface of the sphere).

It is frequently more convenient to describe directly the orientation of the $(\xi, \eta, \xi)$ frame than to describe the rotation as above; i.e., we give directly the angles between the two frames. The general technique is due to Euler, and the three angles which must be specified are known as Euler's angles. There are a great number of different choices which can be made, and indeed have been made by various authors, a freedom which complicates considerably comparisons between different authors. We shall follow here what seems to be the most frequent choice by recent American writers on the subject (Edmonds, 1957 ; Rose, 1957). The position of the $\zeta$ axis will be specified by $\theta$, the angle between $\zeta$ and $z$, and by $\phi$, the angle between $x$ and the projection of $\zeta$ in the $x-y$ plane. These are just the usual spherical coordinates of the unit vector along the $\zeta$ axis in the $(\boldsymbol{x}, \boldsymbol{y}, \boldsymbol{z})$ system. For the third angle, we shall use $\psi$, the angle between $\eta$ and the intersection of the $x-y$ and the $\xi-\eta$ planes. Experimental workers (Dieke and Crosswhite, 1956) have found convenient an alternate choice $\chi$, the angle between $\xi$ and the intersection of the $x-z$ and $\xi-\eta$ planes. These angles are related by the equation $\chi=\chi_{0}+\psi$. where $\tan \chi_{0}=\cos \theta \tan \phi$. If $\theta=90^{\circ}$, then $\chi=\psi$.

A major advantage of Euler's angles is that any given orientation of the $(\xi, \boldsymbol{\eta}, \zeta)$ frame can be obtained by three rotations in succession about the coordinate axes. For these rotations, the matrices (3.5) take on a particularly simple form, and their product, expressing the general form of (3.5) in terms of Euler's angles, may be computed directly. The three rotations are:

1. A rotation by $\phi$ about the $z, \zeta$ axes (which initially coincide). The $\xi-\eta$ plane remains in the $x-y$ plane, but the $\xi-\xi$ and the $\eta-\xi$ planes are rotated by the angle $\phi$.

2. A rotation by $\theta$ about the $\eta$ axis. This will bring the $\zeta$ axis into its final position, as specified by $\phi$ and $\theta$. The $\xi-\eta$ plane will no longer be in the $x-y$ plane, but their intersection is the $\eta$ axis.

3. A rotation by $\psi$ about the $\zeta$ axis. This will bring the $\xi$ and $\eta$ axes to their final orientation.

In performing these rotations, it will be noted that each one is carried out in the $(\xi, \eta, \xi)$ frame, which is generally in a different position each time as a result of the preceding rotation in the sequence. The same final configuration can be obtained by rotating about the $(\boldsymbol{x}, \boldsymbol{y}, \boldsymbol{z})$ frame axes, provided, that the sequence of the angles is reversed. In other words, a rotation of the $(\xi, \eta, \xi)$ frame first about the $z$-axis by the angle $\psi$, then about the $y$ axis by $\theta$, and finally about the $z$-axis again by the angle $\phi$, will yield exactly the same final orientation of the $(\xi, \boldsymbol{\eta}, \xi)$ frame. This may be established either by purely geometric considerations, or by specific multiplication of the matrices involved. Using this latter sequence of angles, we obtain from (3.5)

\section{if}

$\mathbf{u}=(0,0,1), \quad \omega=\phi$ or $\psi$ or $\mathbf{u}=(0,1,0), \quad \omega=\theta$

$$
\mathbf{R}_{\phi}=\left(\begin{array}{ccc}
\cos \phi & -\sin \phi & 0 \\
\sin \phi & \cos \phi & 0 \\
0 & 0 & 1
\end{array}\right) \quad \mathbb{R}_{\theta}=\left(\begin{array}{ccc}
\cos \theta & 0 & \sin \theta \\
0 & 1 & 0 \\
-\sin \theta & 0 & \cos \theta
\end{array}\right) \quad \mathbb{R}_{\psi}=\left(\begin{array}{ccc}
\cos \psi & -\sin \psi & 0 \\
\sin \psi & \cos \psi & 0 \\
0 & 0 & 1
\end{array}\right)
$$

and their product $\mathbf{R}_{\phi} \mathbf{R}_{\theta} \mathbf{R}_{\psi}$ yields the matrices of the representation $D_{1}$ of $\Re_{3}$, now in terms of the three Euler angles,

$$
\mathbb{D}_{1}(\phi, \theta, \psi)=\left(\begin{array}{ccc}
\cos \phi \cos \theta \cos \psi-\sin \phi \sin \psi & -\cos \phi \cos \theta \sin \psi-\sin \phi \cos \psi & \cos \phi \sin \theta \\
\sin \phi \cos \theta \cos \psi+\cos \phi \sin \psi & -\sin \phi \cos \theta \sin \psi+\cos \phi \cos \psi & \sin \phi \sin \theta \\
-\sin \theta \cos \psi & \sin \theta \sin \psi & \cos \theta
\end{array}\right)
$$


Since these matrices are orthogonal, the inverse of a given matrix is its transpose. In terms of $u, \omega$, this amounts to reversing either the vector $u$ or the sign of the angle of rotation. In terms of the Euler angles, the inverse amounts to reversing the sign of the angles and also their sequence. In general, we shall not indicate the variables for $D_{1}$ (or for $D_{N}$ ), since either set may be used.

A third extremely important description of a three-dimensional rotation arises from the CayleyKlein parameters. Let us consider the general $2 \times 2$ matrix with complex elements, which we shall write in the form

$$
\left(\begin{array}{ll}
a e^{i \alpha} & b e^{i \beta} \\
c e^{i(\beta+\gamma)} & d e^{i(\alpha+\delta)}
\end{array}\right)
$$

where $a, b, c$, and $d$ are real nonnegative numbers, and $\alpha, \beta, \gamma$, and $\delta$ are real numbers. This matrix contains eight arbitrary parameters. If we require that it be a unitary matrix $\mathrm{U}$, four conditions of restraint on these parametel's are imposed, and there remain but four independent quantities. These four conditions are: $a=d ; b=c ; a^{2}+b^{2}=1$; and $2 \alpha+\delta=2 \beta+\gamma+\pi$. The further condition that det $\mathrm{U}=+1$ requires that $2 \alpha+\delta=0$, leaving three independent quantities. We can then write

$$
\mathrm{U}=\left(\begin{array}{cc}
a e^{i \alpha} & b e^{i \beta} \\
-b e^{-i \beta} & a e^{-i \alpha}
\end{array}\right),
$$

remembering that $a^{2}+b^{2}=1$. The independent parameters are then, essentially, $a, \alpha$, and $\beta$.

Further, let

$$
\mathbf{R}=\left(\begin{array}{cc}
\zeta & \xi-i \eta \\
\xi+i \eta & -\zeta
\end{array}\right)
$$

be a Hermitian matrix with a trace of zero, $\xi, \eta$, and $\zeta$ being arbitrary real numbers. A unitary transformation

$$
\mathrm{URU}^{-1}=\mathbf{R}^{\prime}
$$

will leave invariant the Hermitian property, the trace, and the determinant, so $\mathbf{R}^{\prime}$ can be written

$$
\mathbf{R}^{\prime}=\left(\begin{array}{cc}
z & x-i y \\
x+i y & -z
\end{array}\right)
$$

Furthermore, $\operatorname{det} \mathbf{R}^{\prime}=-\left(x^{2}+y^{2}+z^{2}\right)=\operatorname{det} \quad \mathbf{R}^{\prime}=$ $-\left(\xi^{2}+\eta^{2}+\zeta^{2}\right)$. If we interpret the sets of numbers $(\xi, \eta, \xi)$ and $(x, y, z)$ as the coordinates of a point in the movable $(\boldsymbol{\xi}, \boldsymbol{\eta}, \zeta)$ system and the fixed $(\boldsymbol{x}, \boldsymbol{y}, \boldsymbol{z})$ system, respectively, we see that the transformation leaves unchanged the distance of the point from the origin, so that the transformation can be interpreted as a rotation. The matrix $\mathbf{U}$ can be explicitly calculated in terms of the Euler angles by finding the matrices $U_{\phi}, U_{\theta}$, and $U_{\psi}$, so that
$\mathbf{U}=\mathbf{U}_{\phi} \mathbf{U}_{\theta} \mathbf{U}_{\psi}$. Thus,

$$
\begin{aligned}
\mathbf{U}_{\phi} & =\left(\begin{array}{cc}
e^{-i \phi / 2} & 0 \\
0 & e^{+i \phi / 2}
\end{array}\right) \\
\mathbf{U}_{\theta} & =\left(\begin{array}{ccc}
\cos \frac{\theta}{2} & -\sin \frac{\theta}{2} \\
\sin \frac{\theta}{2} & \cos \frac{\theta}{2}
\end{array}\right) \quad \mathbf{U}_{\psi}=\left(\begin{array}{cc}
e^{-i \psi / 2} & 0 \\
0 & e^{+i \psi / 2}
\end{array}\right) \\
\mathbf{U} & =\left(\begin{array}{ccc}
e^{\frac{-i(\phi+\psi)}{2}} \cos \frac{\theta}{2} & -e^{\frac{-i(\phi-\psi)}{2}} \sin \frac{\theta}{2} \\
e^{\frac{+i(\phi-\psi)}{2}} \sin \frac{\theta}{2} & e^{\frac{+i(\phi+\psi)}{2}} \cos \frac{\theta}{2}
\end{array}\right)
\end{aligned}
$$

Just as in the previous discussion distinguishing between $\Re_{3}$ and $D_{1}$, we have here an abstract group of unitary unimodular transformations in a complex space of two dimensions, and a particular representation of the group through the matrices (3.7). The abstract group we shall designate as $\mathfrak{U}_{2}$, the particular representation by the collection of $\mathrm{U}$ matrices (3.7) as $D_{1 / 2}$. The elements of the representation $D_{1 / 2}$ may be given either in terms of the Euler angles as in (3.7), or in terms of the unit vector $\mathbb{u}$ and the angle $\omega$, though we shall not give the latter form here explicitly. Since it will not generally be necessary to indicate the variables, we shall generally write the matrices merely as $\mathrm{D}_{1 / 2}$ (instead of $\mathrm{U}$ ) to parallel our earlier distinction between $\mathrm{D}_{1}$ and $D_{1}$.

Let us consider the implication of this transformation in more detail. We first observe that the matrix $\mathbf{R}$ can be written in the form

$$
\mathbf{R}=\xi\left(\begin{array}{ll}
0 & 1 \\
1 & 0
\end{array}\right)+\eta\left(\begin{array}{rr}
0 & -i \\
i & 0
\end{array}\right)+\zeta\left(\begin{array}{rr}
1 & 0 \\
0 & -1
\end{array}\right)
$$

where the $2 \times 2$ matrices are the Pauli matrices corresponding to the $x, y$, and $z$-components, respectively, of the spin angular momentum operator. If we let $(\xi, \eta, \zeta)$ be a unit vector, the matrix $\mathbf{R}$ is the operator corresponding to the projection of the spin angular momentum in the direction given by the unit vector $(\xi, \eta, \zeta)$. Let us consider for simplicity $U$ for $\theta=\psi=0$. $\quad \mathbf{R}^{\prime}=$ URU $\mathbf{U}^{-1}$ may similarly be written

$$
\begin{aligned}
\mathbf{R}^{\prime} & =(\xi \cos \phi-\eta \sin \phi)\left(\begin{array}{ll}
0 & 1 \\
1 & 0
\end{array}\right) \\
& +(\eta \cos \phi+\xi \sin \phi)\left(\begin{array}{rr}
0 & -i \\
i & 0
\end{array}\right)+\xi\left(\begin{array}{rr}
1 & 0 \\
0 & -1
\end{array}\right) .
\end{aligned}
$$

This corresponds to our interpretation that the vector with components $(\xi, \eta, \xi)$, fixed in the $(\xi, \eta, \zeta)$ frame, is given a rotation about the $z-\zeta$ axis, positive in terms of the right hand screw 
convention. $\mathbf{R}^{\prime}$ is now the operator corresponding to the projection of spin in the new direction, still described in the movable system by $(\xi, \eta, \zeta)$, but described in the fixed $(\boldsymbol{x}, \boldsymbol{y}, \boldsymbol{z})$ system by $x=\xi \cos \phi-\eta \sin \phi \quad y=\eta \cos \phi+\xi \sin \phi \quad z=\zeta$. Note that the Pauli matrices retain their form, i.e., they are expressed in the fixed $(\boldsymbol{x}, \boldsymbol{y}, \boldsymbol{z})$ frame. However, we can also write

$$
\begin{aligned}
\mathbf{R}^{\prime}=\xi\left(\begin{array}{rr}
0 & e^{-i \phi} \\
e^{i \phi} & 0
\end{array}\right) \\
+\quad \eta\left(\begin{array}{rr}
0 & -i e^{-i \phi} \\
i e^{i \phi} &
\end{array}\right)+\xi\left(\begin{array}{rr}
1 & 0 \\
0 & -1
\end{array}\right)
\end{aligned}
$$

where $\xi, \eta$, and $\zeta$ retain their original values, and the Pauli spin matrices have been transformed to the form appropriate to the new $\xi, \eta, \zeta$ axes. Recalling that this is a rotation by $+\phi$, this should be cquivalent to a rotation of the physical system, the fixed $x, y, z$ axes, and the spin functions described therein, by $-\phi$. In other words, generally, a transformation of the spin operators $\sigma$, by $\mathbf{U}$ is equivalent to a transformation of the spin functions themsclves by $\mathbb{U}^{-1}$. More formally, consider $\phi=\mathbb{R} \psi$, where $\phi$ and $\psi$ are spin wave functions. This expression, under the transformation $\mathrm{U}$, yields

$$
\mathbf{U} \boldsymbol{\phi}=\mathbf{U R U}^{-1} \mathbf{U}_{\psi} \quad \text { or } \quad \boldsymbol{\phi}^{\prime}=\mathbb{R}^{\prime} \psi^{\prime}
$$

which represents merely the transformation of each side of the expression to a new frame of reference; in other words, essentially the same angular momentum measurement. From the standpoint of a simple rotation of $\mathbb{R}$ to measure angular momentum in the new direction fixed by $\mathbf{R}^{\prime}$ as considered in the original frame of reference, we are interested in the quantity $\mathbb{R}^{\prime} \psi=\mathbf{U} \mathbf{R U}^{-1} \psi$ $=\mathrm{X}$ which is generally different from $\phi$. This new measurement may be transformed by $\mathrm{U}^{-1}$ to give

$$
\mathrm{U}^{-1} \mathbf{X}=\mathbf{U}^{-1}\left[\mathrm{URU}^{-1}\right] \mathrm{UU}^{-1} \psi=\mathbb{R}\left[\mathrm{U}^{-1} \psi\right] .
$$

In other words, the rotation of $\mathbb{R}$ to $\mathbb{R}^{\prime}, \mathbf{U} \mathbf{U}^{-1}$ gives the same result as the inverse transformation of the spin wave functions by $\mathbf{U}^{-1}$. Since we are usually interested in transforming wave functions, we shall write $\mathbf{D}_{1 / 2}=\mathbf{U}^{-1}$ rather than $\mathbf{D}_{1 / 2}=\mathbf{U}$.

The presence of the half-angle functions in this transformation reflects the very special properties of the spin transformations. In particular, the rotation $\phi^{\prime}=\phi+2 \pi, \theta^{\prime}=\theta+2 \pi, \psi^{\prime}=\psi+2 \pi$ clearly yields an orientation of axes identical to that designated by $\phi, \theta, \psi$. On the other hand, it may be seen that $\mathbb{D}_{1 / 2}\left(\phi^{\prime}, \theta^{\prime}, \psi^{\prime}\right)=-\mathbb{D}_{1 / 2}(\phi, \theta, \psi)$. Thus, for each change of orientation in ordinary threedimensional space, thcre are two matrices corresponding to this in the two-dimensional spin space. For this reason, the set of matrices $D_{1 / 2}$ does not constitute a representation of the three-dimen- sional rotation group in the stricted mathematical sense of the term. It has become customary to refer to $D_{1 / 2}$ as a "two-valued" or "ambiguous" representation, and the abstract group $\mathfrak{U}_{2}$ as the double rotation group. If a rotation is specified by giving the initial and final configuration of axes, the matrices $\mathbb{D}_{1 / 2}$ are fixed apart from the sign. The sign may also be fixed if the detailed course of the rotation is also prescribed. Clearly, the matrix $D_{1 / 2}$ is a continuous function of the variables $\phi, \theta, \psi$ and becomes the unit matrix for no rotation at all. In other words, if the initial and final axes are the same, we know only that the appropriate $D_{1 / 2}$ matrix is either the unit $2 \times 2$ matrix or its negative, while the detailed knowledge that no rotation at all has occurred (or one involving a path in the $(\mathbf{u} \omega)$ space of homotopy class $P_{0}$ ) enables us to fix upon the unit matrix as the appropriate one. Generally, if two axes are related by the Euler angles $\phi, \theta, \psi$ we will select $\mathbf{D}_{1 / 2}(\phi, \theta, \psi)$ if the transformation is of homotopy $\operatorname{class} P_{0}$, and $-\mathbf{D}_{1 / 2}(\phi, \theta, \psi)$ if of class $P_{1}$.

\subsection{Group Theory and the Free Atom or Ion}

The Hamiltonian for a free atom contains the following types of terms:

1. Kinetic energy terms $\frac{1}{2 m} \mathbf{p}_{i}{ }^{2}$

2. Central field potential terms $-\frac{Z e^{2}}{r_{i}}+V^{\prime}\left(r_{i}\right)$

3. Electrostatic repulsion terms $\frac{e^{2}}{r_{i j}}-V^{\prime}\left(r_{\imath}\right)$

\section{Spin-orbit interaction terms $\zeta\left(r_{i}\right) \mathbf{1}_{i} \cdot \mathbf{s}_{i}$.}

In the above, the $V^{\prime}\left(r_{i}\right)$ is selected so that the overall effect of the third term is minimized. In addition to the terms of the free ion Hamiltonian, we shall also be interested in the effects of the crystal lattice potential $V$ discussed in the preceding section and of an external magnetic field $\mathbf{B}$. Consequently, we shall be interested in the operations which leave invariant these various terms in the Hamiltonian.

Let us first consider terms (1) and (2) only. In this case, each electron is considered to move independently of the detailed motion of the other electrons present, their mutual interaction appearing in an averaged form in the $V^{\prime}\left(r_{i}\right)$ term. Term (1) gives the Iaplacian operator, which may be viewed as a scalar product of two vector operators and is therefore invariant under an arbitrary rotation of coordinate axes about the force center. Since second derivatives are involved, it is also invariant under the inversion. Similarly, the second term involves only the scalar magnitude of $\mathbf{r}$ which is invariant under the same two operations. The solutions of the Schrodinger equation for one electron for these two terms only are 
characterized by the quantum numbers $n, l, m_{l}$, and $m_{s}$, and have a degeneracy equal to $2(2 l+1)$, there being $2 l+1$ values of $m_{l}$ and 2 values of $m_{s}$ all belonging to the same energy and all related to each other by the particular choice of coordinate axis. These $2 l+1$ functions are just the functions (2.8). Thus, a rotation of the coordinate axes will cause each of the $2 l+1$ functions in the new set of axes to be expressed in terms of all $2 l+1$ functions referred to the old axes, yielding a $2 l+1$ dimensional representation of the rotation as in section 3.2 . It is shown in the general theory of groups that for all nonnegative integral values of $l$ these representations are in fact reps. General formulas for the matrix elements in terms of Euler's angles have been given by Wigner (1931). Specific examples of these matrices using a real basis (2.5) rather than the complex basis (2.8) have already been given in table 6 . It is further to be noted that, for $l>0$, the representation is faithful, i.e., there is a unique matrix corresponding to each rotation. We shall designate the rep by $D_{l}$, its elements by $\mathbf{D}_{l}$.

For a single electron, as in hydrogen or in the alkali-type atoms or ions, term (3) in the Hamiltonian vanishes. So long as term (4) is neglected, the transformation of the $2 l+1$ values of $m_{l}$ and the two values of $m_{s}$ are completely independent of each other, and may eren be described with respect to different coordinate axes. The general transformation may be represented by a matrix of $2(2 l+1)$ dimensions, the direct product of $\mathbf{D}_{1 / 2}$ and $\mathrm{D}_{l}$. Upon the introduction of term (4), the independent transformation of spin and orbit no longer leaves the Hamiltonian invariant. The invariance of the scalar product of two operators (e.g., l.s) is founded upon the assumption that each is referred to the same set of axes. The group of allowed transformations is now less general, since both spin and orbit must simultaneously undergo the same rotation. The $2(2 l+1)$ dimensionsal matrix is still an element of a representation of the group of allowed transformations, but it is no longer irreducible, and it may be transformed by a new choice of wave functions into a diagonal matrix of sub-matrices of dimensionality $2\left(l+\frac{1}{2}\right)+1$ and $2\left(l-\frac{1}{2}\right)+1$. The correct choice of wave funciions is precisely that dictated by the usual transformation from a $m_{l}, m_{s}$ to $j$, $m$, representation in one-electron spectra. The wave functions are determined by the coefficients known variously as Wigner, Clebsch-Gordon, or rector addition coefficients, and yield allowed values of the total angular momentum $j=l+\frac{1}{2}$ or $i=l-\frac{1}{2}$.

A similar argument is applicable to the case of two electrons. Without terms (3) and (4) in the Hamiltonian, all transformations are independent and most generally yield a matrix of $2^{2}\left(2 l_{1}+1\right)$ : $\left(2 l_{2}+1\right)$ dimensions. The introduction of term (3) now excludes those transformations affecting differently the space parts of the two one-electron ware functions, and the space portion of the matrix can be reduced into sub-matrices $\mathrm{D}_{L}$ of dimensionality $2 L+1$, where $L$ ranges from $l_{1}+l_{2}$ to $\left|l_{1}-l_{2}\right|$. The restrictions of the Pauli principle require that the four-dimensional spin matrix be simultaneously reduced, yielding a three-dimensional submatrix $\mathbf{D}_{1}$ and the unit 1 by 1 matrix $\mathbf{D}_{0}$. This reduction of the spin transformation matrix corresponds to the wellknown existence of triplet and singlet states in two-electron spectra. For our immediate purposes, perhaps the most noteworthy point is the disappearance of the ambiguity in sign of the spin transformations. This is a general characteristic of atoms or ions with an even number of electrons. The reps of $\mathbb{H}_{2}$ are infinite in number and are of both eren and odd dimensionality. Those of even dimensionality include $D \frac{1}{2}$ and others designated as $D_{3} / 2, D_{5} / 2$, etc., the dimensionalities being $2,4,6, \ldots$. respectively. These representations are all faithful, and hence all are double-valued representations of the three-dimensional rotation group. The odd dimensional reps are the $D_{0,1,2,3}$. . previously discussed as reps of the three-dimensional rotation group, but these latter are not faithful. Aside from the identity rep $D_{0}$, any element $\mathbf{D}_{J}, J$ integral, corresponds to $\pm \mathbf{D}_{J}$, $J$ half-integral. This is a point of greatest importance when considering the representations of the point groups, as we shall see shortly.

The introduction of term (4) into the Hamiltonian now requires the reduction of the $(2 S+1)$. $(2 L+1)$ dimensional direct product into a diagonal array of sub-matrices $\mathrm{D}_{J}$, where $J=L+S$, $L+S-1, \ldots|L-S|$. Here, the integral $S$ yields integral $J$ and the spin ambiguity remains suppressed. In certain atoms, the magnitude of the various terms in the Hamiltonian requires that term (4) be introduced before (3). The reduction of the various matrices must then be accomplished in a different order, corresponding to the $j-j$ coupling scheme rather than the $L-S$ scheme discussed above. For 3 or more electrons, the details can become increasingly complicated, but the general features remain the same. In particular, integral $J$ 's without spin ambiguity appear for systems with an even number of electrons, while half-integral $J$ 's corresponding to double-valued representations of pure rotations appear for systems with an odd number of electrons.

There is another symmetry which must be considered at this point, that of the inversion. Terms (1), (2), (3) in the Hamiltonian depend only on the length of a vector or vector operator and hence are invariant under the transformation from a right-handed to a left-handed coordinate system. The angular momentum operator, orbital or spin, has the transformation properties of a cross product of two vectors, i.e., an axial vector or pseudovector. Although the two vectors will change sign on inversion, their product will not and the vector operators (pseudovectors) 1 and $\mathbf{s}$ as well as the Hamiltonian term $1 . \mathrm{s}$ are also invariant. It is 
important to note at this point the distinction between a vector and its description. Let us consider the vector $\mathbf{r}=(x, y, z)$ and $\mathbf{t}=(u, v, w)$, with $\mathbf{r} \times \mathbf{t}=(y w-z v, z u-x w, x v-y u)$. If we now switch to a left-handed system, the vector 1 remains the same, but it is now described by $(-x,-y,-z)$. Likewise, $t$ remains the same but its description becomes the negative of the original. On the other hand, the product $\mathbf{r} \times \mathbf{t}$ retains the same description, but is consequently a different vector since it is now in a left-handed system. The distinction between a quantity and its description is a fundamental one which must on occasion be made very carefully. If we consider a one-electron wave function $\psi(x, y, z, s)$, the description of this state in the inverted frame is $\psi(-x,-y,-z$, $s)$, the description of an angular momentum being invariant. $\psi$ is a homogeneous polynomial of degree $l$ in the variables $x, y, z$ and the transformation under the inversion is that of $(-1)^{l}$ and that of a $n$-electron system $(-1)^{2 l i}$. Strictly speaking, of course, $\Sigma l_{i}$ will not have a definite numerical value when terms (3) and (4) in the Hamiltonian are introduced, but only down through term (2). Nevertheless, the introduction of (3) and (4), invariant under the inversion, will admix only wave functions of the same value of $(-1)^{\Sigma l_{i}}$ which may be used to describe states of the free atom or ion. In addition to $J$, the states may be designated even or odd, ( $g$ or $u$, respectively) depending on $(-1)^{\Sigma l_{i}}$.

\subsection{Group Theory and the Perturbed Ion}

It is the primary purpose of this paper to consider in detail the effects of terms in the Hamiltonian arising from the crystal lattice perturbation $V$ and from the external magnetic field $\mathbf{B}$. There are two techniques which may be used in the solution of the problem, both of which must be used in a complementary fashion for a complete discussion. One technique is to apply further the theory of groups, reducing the representation of the state of the free ion according to the reps of the appropriate electrostatic-magnetic field symmetry group. This technique will yield the number of levels, their description in terms of their behavior under the allowed symmetry operations, and selection and polarization rules for various types of transitions. The method will not yield purely numerical details, such as the relative positions of levels, their variation with electric and magnetic field intensities, or transition probabilities (save for the vanishing forbidden transitions). The other technique is to calculate to some degree of approximation the matrix elements of the perturbing potentials in terms of the free ion wave functions, interpreting the results in the framework of the usual perturbation theory. In the final analysis, this latter procedure will yield not only the numerical details not obtained from the former technique, but can be so interpreted as to yield all of the results that the former technique will give. Nevertheless, the group-theoretical ap- proach is one of great generality and provides a very concise systematic framework for stating the results obtained. Furthermore, the principles of group theory (or essentially similar arguments) are used to determine what matrix elements of the perturbing potentials vanish if the second approach is used, and to calculate the magnitude of those which do not vanish. Hence, the two approaches are not really so divergent as might at first sight appear to be the case. In this work, the contributions to the problem which can be made with the use of group theory will be studied in detail before proceeding to the alternate considerations of calculations involving specific matrix elements. While it is not the intent of this paper to furnish a general treatise on group theory, and a general acquaintance with at least the basic definitions will be presumed, certain concepts will be examined in detail and applied as the necessity arises.

Let us consider the very simple group $\mathrm{C}_{3}$, consisting of the elements $\mathbb{C}_{3}, \mathbf{C}_{3}^{2}$, and $\mathbb{E}$. We shall describe the group in terms of the effect of its operations on the basis triangle of figure $2 \mathrm{a}$, taking $\mathrm{G}_{3}$ as a counterclockwise rotation of the triangle by $120^{\circ}$. In the present configuration, the operation $\mathbb{C}_{3}$ will cause the vertex (1) to appear at the point previously occupied by. (2), (2) at that occupied by (3), and also (3) $\rightarrow(1)$. We may shorten this to $\mathrm{G}_{3}:(1) \rightarrow(2) \rightarrow(3)$. We may also describe the operations in terms of their effect on the triangle in figure $2 \mathrm{~b}$, where we see that we also obtain $\mathrm{G}_{3}:(1) \rightarrow(2) \rightarrow(3)$. Notice that figure $2 \mathrm{~b}$ differs from figure $2 \mathrm{a}$ only in that $\mathbb{C}_{3}$ has been applied to the basis triangle before we use it to describe the group. We could also have applied $\mathrm{C}_{3}^{2}$ to figure $2 \mathrm{a}$ before describing the operation, and again would have obtained $\mathbf{C}_{3}$ : $(1) \rightarrow(2) \rightarrow(3)$. A study of the operation $\mathbf{C}_{3}^{2}$ will show that, using any of these basis triangles, we obtain $\mathbb{C}_{3}^{2}:(1) \rightarrow(3) \rightarrow(2)$ as its description. Thus, if we restrict ourselves to transformations within the group under discussion, there is no transformation which will cause $\mathbf{C}_{3}^{2}$ and $\mathbf{C}_{3}$ to have the same description in terms of their effect on the basis triangle. Let us now consider the group $\mathrm{D}_{3}$, where we have added to our original set of three elements three rotations of $180^{\circ}$ about axes in the $x-y$ plane, described in terms again of figure 2 a as $\mathbb{C}_{2}:(2) \rightarrow(3) ; \mathbb{C}_{2}^{\prime}:(1) \rightarrow(2)$; and $\mathbf{C}_{2}^{\prime \prime}:(1) \rightarrow(3)$. Still restricting ourselves to transformations within the group, let us consider the effect of $\mathrm{C}_{3}$ in terms of the basis of figure $2 \mathrm{c}$, derived from figure $2 \mathrm{a}$ by the application of $\mathbf{C}_{2}$. Here we see that we obtain $\mathrm{C}_{3}:(1) \rightarrow(3) \rightarrow(2)$, but this is the same as $\mathbf{C}_{3}^{2}$

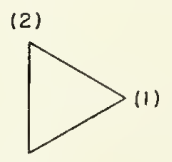

(3) a (2)

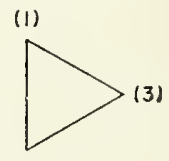

(2)

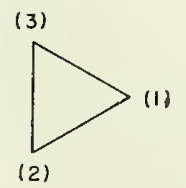

b

6
Figure 2 
described in terms of figure $2 \mathrm{a}$. We thus see that in our first case $\mathbf{C}_{3}$ and $\mathbf{C}_{3}^{2}$ were essentially different, in that none of our allowed transformations permit them to hare the same description, while in the second case at least part of this essential difference has been lost. This difference is expressed by the notion of class. In the first case $\mathrm{C}_{3}$ and $\mathrm{C}_{3}^{2}$ are said to be in a different class, while in the second case they are said to be in the same class.

More generally, if we consider the general transformation, $\mathbf{P}^{\prime}=$ TPT $^{-1}$ and limit ourselves to transformations $\mathbf{T}$ belonging to the group of $\mathbf{P}$, we see that $\mathbf{P}^{\prime}$ is also in the group of $\mathbf{P}$, and is defined as being in the class of $\mathbf{P}$. For each $\mathbf{T}$ of the group a $\mathbf{P}^{\prime}$ is obtained, not necessarily distinct, and the complete set of $\mathbf{P}^{\prime}$ constitutes the class of $\mathbf{P}$. Clearly, if $\mathbf{P}$ and $\mathbf{T}$ commute, $\mathbf{P}^{\prime}=\mathbf{P}$. In an Abelian group, where all elements commute, every element is in a class by itself. Conversely, if $\mathbf{P}^{\prime}$ is distinct from $\mathbf{P}$, then $\mathbf{P}$ and $\mathbf{T}$ must not commute. The separation of a group into classes may be accomplished either by geometric considerations such as outlined abore, or by formal computation. The formal computation is aided by the following considerations. Let $\mathrm{N}$ be the set of group elements which commute with P. It may be shown that this set of elements is in fact a subgroup (possibly the entire group) called the normalizer of P. Any element of $\mathrm{N}$ as the transforming element yields a $\mathbf{P}^{\prime}=\mathbf{P}$. Let $\mathbf{K}$ be a group element not in $\mathbf{N}$ ( $\mathbf{K}$ does not exist if the normalizer is the whole group). Then $\mathbf{K}$ as the transforming element $\mathbf{T}$ yields a $\mathbf{P}^{\prime}$ different from $\mathbf{P}$, but any element in the complex $\mathbf{K N}$ yields the same $\mathbf{P}^{\prime}$ as does $\mathbf{K}$. Similarly, if $\mathbf{L}$ is a group element not in $\mathrm{N}$ or $\mathrm{KN}$, it will yield still another $\mathbf{P}^{\prime \prime}$, but all elements in LN will yield the same $\mathbf{P}^{\prime \prime}$. Generally, if the group order is $g$ and the order of the normalizer is $n$, the ratio $g / n$, an integer, is called the index of $\mathrm{N}$, and the group may be split into $\mathrm{g} / \mathrm{n}$ complexes, one of which is the subgroup $\mathrm{N}$. All of the elements of the class of $\mathbf{P}$ may then be obtained by transforming $\mathbf{P}$ with an element from each of these $g / n$ complexes. Hence the class of $\mathbf{P}$ has $g / n$ distinct elements.

These considerations may be applied to the group $D_{3}$ discussed above as an example. The group order is 6 , and the elements which commute with $\mathrm{C}_{3}$ are $\mathrm{E}, \mathrm{C}_{3}, \mathrm{C}_{3}^{2}$. Hence the index of the normalizer is 2 , and there are two elements in the class of $\mathbf{C}_{3}$, one of which is $\mathbb{C}_{3}$ itself, the other of which may be obtained by transforming $\mathbf{C}_{3}$ with any one of $\mathbf{C}_{2}, \mathbf{C}_{2}^{\prime}$, or $\mathrm{C}_{2}^{\prime \prime}$. To accomplish this latter we need the multiplication table for the group elements, usually summarized as $\mathrm{C}_{3}^{3}=\mathrm{C}_{2}^{2}=\mathrm{E}$, $\mathrm{C}_{3} \mathrm{C}_{2}=\mathrm{C}_{2} \mathrm{C}_{3}^{2}$. The other element in the class of $\mathrm{C}_{3}$ is $\mathrm{C}_{2} \mathrm{C}_{3} \mathrm{C}_{2}^{-1}=\mathrm{C}_{2} \mathrm{C}_{3} \mathrm{C}_{2}=\mathrm{C}_{2} \mathrm{C}_{2} \mathrm{C}_{3}^{2}=\mathrm{C}_{3}^{2}$, as was geometrically shown abore. $\mathrm{C}_{2}$ commutes only with itself and $\mathbf{E}$, so the order of its normalizer is 2 , its index is 3 , and there are three elements in the class of $\mathbf{G}_{2}$. These are $\mathbf{C}_{2}$ and the products of $\mathbf{C}_{2}$ with $\mathbf{C}_{3}$ and $\mathbf{C}_{3}^{2}$.

If we have a set of $g$ unitary matrices forming a representation of a group of order $g$, the set of traces of these $g$ matrices is said to be the character of the representation. The importance of the concept lies in the invariance of the trace under a unitary transformation, $\mathbf{T} r \mathbf{P}^{\prime}=\mathbf{T} r \quad \mathbf{U P U}^{-1}=$ $\operatorname{Tr} \boldsymbol{P}$. We see immediately that if $\mathbf{U}$ is a matrix of the representation, then $\mathbf{P}^{\prime}$ is an element of the class of $\mathbf{P}$ and all elements of the same class have the same trace. If $U$ is a matrix reducing the representation into two or more reps, the character of the representation is the sum of the characters of the component reps. This latter fact will allow us to determine what reps may be present in a given representation without actually finding the particular U necessary to accomplish the reduction.

This latter procedure is precisely what we have outlined above in sketching the application of group-theoretical ideas to the description of states of free atoms or ions, and what we wish to examine in detail when considering the effect of the introduction of the crystal field $V$ and the external magnetic field $\mathbf{B}$. Before the introduction of the external fields, the Hamiltonian is invariant under arbitrary rotation (of the simple or double group) and inversion, and the wave functions are transformed by $\mathrm{D}_{J}$ and $(-1)^{\Sigma l_{i}}$, respectively. After the introduction of the external fields, the Hamiltonian is now invariant only with respect to subgroups of the original group of transformations. The original rep of the complete group will not in general be a rep of this smaller group but merely a representation, reducible into reps of the smaller group. This is the expression of the fact that the original $2 J+1$ fold degenerate level will under the influence of these external fields split into several levels of lower degeneracy. The character of the original representation is the sum of the characters of the reps into which the state splits. We shall now outline the method whereby this determination of the component reps may be accomplished.

\subsection{Group Characters and the Rep- $D_{J}$}

Let us consider a finite group of $g$ elements, with the elements separated into classes. There will, in general, be several reps for this group. Let us write the trace in the $i$-th rep and $j$-th class as $\chi_{i j}$. For a given $i, j$ will assume values from 1 to $n$, where $n$ is the number of classes, and $\chi_{i j}$ will be a set of numbers, generally complex and not necessarily all distinct. Strictly speaking, the set of numbers $\chi_{i j}$ for all $j$ and a given $i$ constitutes the character, while the number $\chi_{i}$ for a particular $i$ and $j$ is merely the trace of a matrix, and one component of the character. This may be emphasized by considering the character as a vector $\chi_{t}$ in a space of $n$ dimensions, with the $n$ components, $x_{i j}$. This is a distinction which is usually either overlooked or ignored. We shall now introduce a weighted trace, $\xi_{i j}$ defined as $\sqrt{\frac{N_{j}}{g}} \chi_{i j}$, where $N_{j}$ is the number of elements in the class $j$, and, correspondingly, a weighted character $\xi_{i}$. Fundamental to our further considera- 
tions will be the orthogonality theorem for these weighted characters, which we shall state without proof, referring to Wigner (1931) for details. This theorem states the following:

1. The weighted character $\xi_{i}$ for a given $i$ is a normalized vector in the Hermitian sense in a space of $n$ dimensions. Symbolically

$$
\xi_{i} \cdot \xi_{i}=\sum_{j=1}^{n} \xi_{i j}^{*} \xi_{i j}=1
$$

2. The weighted characters belonging to different reps are orthogonal in the Hermitian sense.

$$
\xi_{i} \cdot \xi_{k}=\sum_{j=1}^{n} \xi_{i j}^{*} \xi_{k j}=\delta_{i k}
$$

3. The set of weighted characters $\xi_{i}$ for all $i$ forms a complete set in the space of $n$ dimensions, in terms of which the weighted character of any representation may be expanded. If $\boldsymbol{\Xi}$ is such a weighted character, with components $\Xi_{j}=\sqrt{\frac{N_{j}}{g}} \mathrm{X}_{j}$,

$$
\Xi=\sum_{i=1}^{n} a_{i} \xi_{i} \text { and } a_{i}=\xi_{i} \cdot \Xi^{\circ} \cdot
$$

Two important conclusions may be immediately drawn from this theorem. We observe that, since the weighted characters form a complete set in a space of $n$ dimensions, there must be $n$ of them. In other words, the index $i$ as well as $j$ must assume values from 1 to $n$, and the number of reps is equal to the number of classes of the group. The orthogonality theorem suggests that we consider these weighted characters as the elements of a $n \times n$ matrix. The orthonormality (3.8) and (3.9) in the rows (i.e., in the index $i$ ) implies a similar relation in the columns (i.e., in the index $j$ ). In

particular, we have $\sum_{i=1}^{n} \xi_{i E}^{*} \xi_{i E}=1$

where $j=E$ refers to the class of the unit element. The unit element is always in a class by itself, so $N_{E}=1$. The matrix corresponding to the unit element in any rep is the unit matrix of the dimensionality $d_{i}$ of the rep, so $\chi_{i E}=d_{i}$ and $\xi_{i E}=\frac{d_{i}}{\sqrt{g}}$. Consequently,

$$
\sum_{i=1}^{n} \xi_{i E}^{*} \xi_{i E}=1 \quad \text { implies } \quad \sum_{i=1}^{n} d_{i}^{2}=g \cdot
$$

For the finite groups under consideration, there will be a unique solution to this relation in terms of integral values of $d_{i}$. The dimensionality of the reps (and hence the maximum allowable essential degeneracy) will be determined by the order and class structure of the group. Thus, for the group $\mathrm{D}_{3}$ discussed above, with six elements and three classes, we have $2^{2}+1+1=6$, and the maximum essential degeneracy will be 2

We shall begin our discussion of the character of specific reps by considering the reps $D_{J}$, initially limiting $J$ to 1 . The ideas of class, character, and orthogonality just discussed were with specific reference to finite groups, but are applicable to infinite groups with but minor modification, the most far-reaching being in the orthogonality relations. In this case the finite sums cited are replaced by integrals over parameters defining the elements of the group. We shall not require the theorem for infinite groups to be discussed here.

A matrix $\mathbb{D}_{1}$ expresses a transformation of a vector $(\xi, \eta, \zeta)$ into a vector $\mathbf{r}^{\prime}(x, y, z)$. The transformation is orthogonal, leaving the length of the vector unchanged, and may be viewed as a rotation about an axis along $r \times \mathbf{r}^{\prime}$ with an angle of rotation $\omega$ such that $\cos \omega=\frac{\mathbf{r} \cdot \mathbf{r}^{\prime}}{|\mathbf{r}|\left|\mathbf{r}^{\prime}\right|}$. To resolve the group elements into classes note that we can first rotate the vector $\mathbf{r}$ into the $x-y$ plane and, if necessary, perform a rotation about $r$ so that $\mathbf{r}^{\prime}$ will also lie in the $x-y$ plane. We thus transform $\mathrm{D}_{1}$ into a rotation about the $z$ axis where it will, from (3.5) for $\mathbb{U}=(0,0,1)$ assume the form

$$
\mathbb{R}_{1}(\omega)=\left(\begin{array}{ccc}
\cos \omega & -\sin \omega & 0 \\
\sin \omega & \cos \omega & 0 \\
0 & 0 & 1
\end{array}\right)
$$

We see that all rotations with the same angle of rotation are in the same class, and that the trace is $1+2 \cos \omega$. It is important to observe that the fundamental interval of $\omega$ and the complete range of the character, here a vector with an infinite number of components, may be obtained with $\omega$ between the limits 0 and $\pi$. This reflects the fact that a rotation, $-\omega$ about a given axis is equivalent to a rotation $\omega$ about an oppositely directed axis. Thus every rotation is in the same class as its inverse.

Preparatory to considering other integral values of $J$, let us transform $\mathbb{R}_{1}(\omega)$ to the purely diagonal form $\mathbb{R}_{1}^{\prime}(\omega)$ given by

$$
\mathbf{R}_{1}^{\prime}(\omega)=\left(\begin{array}{ccc}
e^{+i \omega} & 0 & 0 \\
0 & 1 & 0 \\
0 & 0 & e^{-i \omega}
\end{array}\right)
$$

This is the matrix describing the effect of a rotation about the $z$ axis on the three states $(+1,0$, -1 ) of a $p$ electron. For a $d$ electron, we know 
that such a rotation may be described by the matrix

$$
\mathbf{R}_{2}^{\prime}(\omega)=\left(\begin{array}{lllll}
e^{+2 i \omega} & 0 & 0 & 0 & 0 \\
0 & 0^{+i \omega} & 0 & 0 & 0 \\
0 & 0 & 1 & 0 & 0 \\
0 & 0 & 0 & e^{-i \omega} & 0 \\
0 & 0 & 0 & 0 & e^{-2 i \omega}
\end{array}\right) .
$$

Since there is a unique 1 to 1 correspondence between the matrices $\mathbf{D}_{1}$ and $\mathbf{D}_{2}$, it follows that any matrix $\mathrm{D}_{2}$ may be brought into the form $\mathbf{R}_{2}^{\prime}(\omega)$ by the transformation corresponding to that bringing $\mathrm{D}_{1}$ into $\mathbf{R}_{1}^{\prime}(\omega)$. Here the trace is $1+2 \cos \omega+2$ $\cos 2 \omega$. A similar argument holds for other integral $J$. A general expression $\chi_{J}(\omega)$ is then given by $\sum_{-J}^{J} e^{i n \omega}$. This is a geometrical progression in which the first term is $e^{-i J \omega}$, the common ratio is $e^{i \omega}$, and which contains $2 J+1$ terms. The general formula for the sum of such a progression gives

$$
\frac{e^{-i J \omega}}{1-e^{i \omega}}\left(1-e^{i(2 J+1) \omega}\right)=\chi_{J}(\omega)
$$

which may be simplified to

$$
\chi_{J}(\omega)=\frac{\sin (2 J+1) \frac{\omega}{2}}{\sin \frac{\omega}{2}} .
$$

We see from an inspection of this formula that the relations considered for $D_{1}$ hold generally for integral $J$, namely, $\chi(\omega)=\chi(-\omega)$, and that $\omega$ on the interval $(0, \pi)$ covers the allowed range.

Let us now consider the group for $\mathcal{J}$ halfintegral, initially limiting ourselves to $J=\frac{1}{2}$. Any unitary matrix may be diagonalized, and $\mathrm{D}_{\frac{1}{3}}$ will assume the particular form $\left(\begin{array}{ll}a & 0 \\ 0 & a^{*}\end{array}\right)$ where $|a|$ is +1 . This diagonalized form of $\mathbf{D}_{\hat{1}}$ can be written as

$$
\left(\begin{array}{ll}
e^{+i \frac{\omega}{2}} & 0 \\
0 & e^{-i \frac{\omega}{2}}
\end{array}\right) .
$$

By equating the trace of this matrix to that of the nondiagonalized matrix (3.7), we obtain

$$
2 \cos \frac{\omega}{2}=2 \cos \left(\frac{\phi+\psi}{2}\right) \cos \frac{\theta}{2},
$$

a relation between the angle of rotation $\omega$ and the Euler angles $\phi, \theta, \psi$. We see that in this group, as well as in that with integral $J$, every element and its inverse are in the same class, since the character does not depend on the sign of $\omega$ and that the classes are determined by the parameter $\omega$. The character of this rep is $2 \cos \omega / 2$. The range of the parameter $\omega / 2$ in defining the group element is $(0, \pi)$ corresponding to $\omega$ in three dimensions on the interval $(0,2 \pi)$, again confirming the double nature of this group in describing real rotations. Let $\omega_{0}$ be the angle, less than $\pi$, relating two orientations of three-dimensional axes, the corresponding diagonalized two-dimensional matrix being

$$
\mathbf{R}_{\Sigma}\left(\omega_{0}\right)=\left(\begin{array}{cc}
e^{i \frac{\omega_{0}}{2}} & 0 \\
0 & e^{-i \frac{\omega_{0}}{2}}
\end{array}\right)
$$

with character $2 \cos \omega_{0} / 2$. The other twodimensional matrix corresponding to the same orientation of three-dimensional axes is then given by

$$
\begin{array}{r}
\mathbf{R}_{\hat{2}}\left(\omega_{0}+2 \pi\right)=\left[\begin{array}{cc}
e^{i}\left(\frac{\omega_{0}}{2}+2 \pi\right) & 0 \\
0 & \left.e^{-i\left(\frac{\omega_{0}}{2}+2 \pi\right.}\right)
\end{array}\right) \\
=\left(\begin{array}{cc}
-e^{+i \frac{\omega_{0}}{2}} & 0 \\
0 & -e^{-i \frac{\omega_{0}}{2}}
\end{array}\right)=-\mathbb{R}_{\frac{1}{2}\left(\omega_{0}\right) .}
\end{array}
$$

The character of $\mathbb{R}_{3}\left(\omega_{0}+2 \pi\right)=2 \cos \left(\omega_{0} / 2+\pi\right)=$ $-2 \cos \omega_{0} / 2$. Hence these two elements of $D_{\frac{1}{2}}$ corresponding to the same three-dimensional rotation have different traces and belong to different classes, unless the trace is zero. This latter occurs for $\omega_{0}=\pi$, at which point the two matrices become

$$
\mathbf{R}_{\mathbf{2}}(\pi)=\left(\begin{array}{cc}
i & 0 \\
0 & -i
\end{array}\right) \text { and } \mathbf{R}_{\mathbf{2}}(3 \pi)=\left(\begin{array}{cc}
-i & 0 \\
0 & i
\end{array}\right) \text {. }
$$

For real rotations of $\pi$, the two elements are in the same class, because they are then reciprocal elements.

For higher half-integral values of $J$, with a unique 1 to 1 correspondence with the elements of the group $D_{\frac{1}{2}}$, a similar reduction to diagonal form must be obtainable, yielding

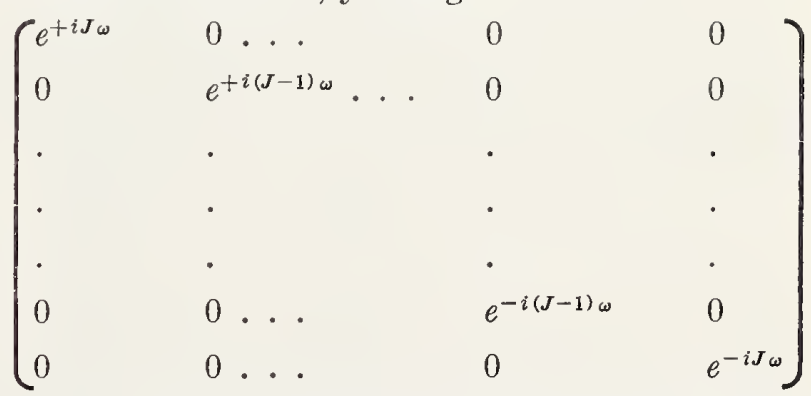

The trace of this matrix is a geometrical progression of the same form as the earlier one for integral $J$, so the general formula (3.12) will still be valid. Values of $\chi_{J}(\omega)$ have been calculated for $\omega$ and $J$ of interest and are presented in table 7 . In further confirmation of previous discussion, for integral $J, \omega / 2$ need only range from 0 to $\pi / 2$, since the numerator is an odd polynomial of the denominator, and the numerator and denominator will change sign at the same time. For half 
TABLE 7. A character table for the reps $D_{\boldsymbol{J}}$

\begin{tabular}{l|r|r|r|r|r|r|r|r|r}
\hline$J$ & $0^{\circ}$ & $\begin{array}{r}60^{\circ} \\
2 \pi / 6\end{array}$ & $\begin{array}{r}90^{\circ} \\
2 \pi / 4\end{array}$ & $\begin{array}{r}120^{\circ} \\
2 \pi / 3\end{array}$ & $\begin{array}{r}180^{\circ} \\
\pi\end{array}$ & $\begin{array}{r}240^{\circ} \\
4 \pi / 3\end{array}$ & $\begin{array}{r}270^{\circ} \\
6 \pi / 4\end{array}$ & $\begin{array}{c}300^{\circ} \\
10 \pi / 6\end{array}$ & $\begin{array}{c}360^{\circ} \\
2 \pi\end{array}$ \\
\hline 0 & 1 & 1 & 1 & 1 & 1 & 1 & 1 & 1 & 1 \\
$1 / 2$ & 2 & $\sqrt{3}$ & $\sqrt{2}$ & 1 & 0 & -1 & $-\sqrt{2}$ & $-\sqrt{3}$ & -2 \\
1 & 3 & 2 & 1 & 0 & -1 & 0 & 1 & 2 & 3 \\
$3 / 2$ & 4 & $\sqrt{3}$ & 0 & -1 & 0 & 1 & 0 & $-\sqrt{3}$ & -4 \\
2 & 5 & 1 & -1 & -1 & 1 & -1 & -1 & 1 & 5 \\
$5 / 2$ & 6 & 0 & $-\sqrt{2}$ & 0 & 0 & 0 & $\sqrt{2}$ & 0 & -6 \\
3 & 7 & -1 & -1 & 1 & -1 & 1 & -1 & -1 & 7 \\
$7 / 2$ & 8 & $-\sqrt{3}$ & 0 & 1 & 0 & -1 & 0 & $\sqrt{3}$ & -8 \\
4 & 9 & -2 & 1 & 0 & 1 & 0 & 1 & -2 & 9 \\
$9 / 2$ & 10 & $-\sqrt{3}$ & $\sqrt{2}$ & -1 & 0 & 1 & $-\sqrt{2}$ & $\sqrt{3}$ & -10 \\
5 & 11 & -1 & 1 & -1 & -1 & -1 & 1 & -1 & 11 \\
$11 / 2$ & 12 & 0 & 0 & 0 & 0 & 0 & 0 & 0 & -12 \\
6 & 13 & 1 & -1 & 1 & 1 & 1 & -1 & 1 & 13 \\
$13 / 2$ & 14 & $\sqrt{3}$ & $-\sqrt{2}$ & 1 & 0 & -1 & $\sqrt{2}$ & $-\sqrt{3}$ & -14 \\
7 & 15 & 2 & -1 & 0 & -1 & 0 & -1 & 2 & 15 \\
$15 / 2$ & 16 & $\sqrt{3}$ & 0 & -1 & 0 & 1 & 0 & $-\sqrt{3}$ & -16 \\
8 & 17 & 1 & 1 & -1 & 1 & -1 & 1 & 1 & 17 \\
\hline & & & & & & & & \\
\hline
\end{tabular}

integral $J$, the numerator will be a product of an odd function of $\sin \omega / 2$ and an odd function of $\cos \omega / 2$. No new values are obtained by going to negative values of $\omega$, since again the numerator and denominator will both change sign together at $\omega=0$. However, the odd terms in $\cos \omega / 2$ change sign at $\omega / 2=\pi / 2$, so that the range $(0,2 \pi)$ in $\omega$ will give distinct traces in this latter case. This is due, of course, to the fact that the division between the two homotopy classes of $D_{1}$ occurs at $\omega=\pi$ rather than at $\omega=0$.

\subsection{Noncubic Grystallographic Groups}

We shall now consider the details of the subgroups of $D_{J}$ in which we shall be interested. Upon the introduction of the crystal field and/or the external magnetic field the general rotationinversion invariance of the free ion Hamiltonian is destroyed and only those transformations which leave invariant terms representing these external fields are admissible. 'These were defined in section 2 in terms of the elements $\mathbb{D}_{1} \times \mathbb{I}$. Because of the unique 1 to 1 correspondence between $\mathbb{D}_{1} \times \mathbb{I}$ and $\mathbb{D}_{J} \times \mathbb{I}$ for integral $\mathscr{J}>0$, the structure as a group of the allowed transformations for all integral $J>0$ is the same as those of the perturbing fields. This correspondence does not hold for $J$ half-integral. In this case we again select out of $\mathbf{D}_{J} \times \mathbb{I}$ those transformations which correspond in terms of $\mathbf{D}_{1} \times \mathbb{I}$ to invariance of the perturbing fields. However, there are two elements of $\mathrm{D}_{J} \times \mathbb{I}, J$ half-integral, corresponding to the same transformation in terms of $\mathbb{D}_{\mathbf{1}} \times \mathbb{1}$, so there will be twice as many elements in the corresponding subgroups. For this reason the structure of the subgroups for half-integral $J$ will be different from those for integral $J$ and must be considered separately. We shall initially limit ourselves to those subgroups appropriate for integral $J$.

We shall examine more closely the detailed structure of those groups listed in table 3 , excluding temporarily the infinite groups listed at the bottom of the table and the cubic point groups. These we divide into four general types:

1. The eyclic groups $\mathrm{C}_{n}$

2. The dihedral groups $\mathrm{D}_{n}$

3. The holohedric groups $\mathrm{C}_{n} \times \mathrm{I}, \mathrm{D}_{n} \times \mathrm{I}$

4. The hemihedric groups, which are the subgroups of the holohedric groups which do not contain $\mathrm{I}$ alone but only in products with rotations.

The cyclic groups $\mathrm{C}_{n}$ are Abelian, of order $n$. The elements of the group are the various powers of $\mathrm{G}_{n}$ with $\mathrm{C}_{n}^{n}=\mathbf{E}$. Every element is in a class by itself; consequently, there are $n$ reps for the group.

The dihedral groups $\mathrm{D}_{n}, n>1$, are of order $2 n$, and include the $n$ powers of $\mathbf{G}_{n}$ and products of these $n$ elements with the elements $\mathrm{C}_{2}{ }^{\prime}$. Products of $\mathrm{C}_{n}$ and $\mathrm{G}_{2}^{\prime}$ are related by $\mathbf{C}_{2}^{\prime} \mathrm{C}_{n}=\mathbf{G}_{n}^{-1} \mathrm{C}_{2}^{\prime}$. Hence the group in non-Abelian for $n>2$. If $n=2$, every element is in a class by itself, otherwise the class structure is more complicated. For $n>2$, the element $\mathbb{C}_{n}^{k}$ and its inverse $\mathbb{C}_{n}^{n-k}$ are in the same class. If $n$ is odd, there are $n-1$ powers of $\mathbf{C}_{n}$ (eliminating the unit element in a class by itself) which are grouped by pairs into $\frac{n-1}{2}$ classes. 
The $n$ products of $\mathbf{C}_{2}^{\prime}$ with the powers of $\mathrm{C}_{n}$ are all in the same class, so we have a total of

$$
2+\frac{n-1}{2}=\frac{n+3}{2}
$$

classes. For $n>2$ and even, the element $\mathrm{C}_{n}^{n / 2}$ is its own inverse and like $\mathbf{E}$ is in a class by itself. The other $n-2$ powers of $\mathbf{C}_{n}$ fall into $\frac{n-2}{2}$ classes, each with two elements. The $n$ products of the form $\mathrm{C}_{n}^{k} \mathrm{C}_{2}^{\prime}$ will in this case fall into two classes, one with $k$ even, the other with $k$ odd. Here the total number of classes is given by $2+\frac{n-2}{2}+2=3+\frac{n}{2}$.

The case for $n=3$ has been discussed above (section 3.4) in detail in connection with the definition of class. In the present work, this is the only case we shall be concerned with, though a little geometric consideration on the symmetry of the pentagon, heptagon, etc, will confirm the above discussion of odd $n$. Since $n$ is odd, an axis of the form $\mathrm{C}_{2}^{\prime}$ will pass through a vertex and the opposite side of a $n$-gon. For $n$ even, such axes will join either opposite pairs of vertices or sides, and no operation of the group either represents a rotation about a line joining a vertex and a side, or will transform a rotation about one type of axis into one about the other.

The holohedric groups $\mathrm{D}_{n} \times \mathrm{I}, \mathrm{C}_{n} \times \mathrm{I}$ contain twice as many elements as $\mathrm{D}_{n}, \mathrm{C}_{n}$ and hence are of order $4 n$ or $2 n$, respectively. Since I commutes with all elements, the group $\mathrm{C}_{n} \times \mathrm{I}$ is Abelian. It is interesting to note that for odd $n$ it is also cyclic since the group can be written in terms of the powers of $\mathbf{C}_{n} \mathbf{I}$, with $\left(\mathbf{I C}_{n}\right)^{n}=\mathbf{I}$. In the case of $\mathrm{D}_{n} \times \mathrm{I}$, if $\mathbf{A}, \mathbf{B}$, and $\mathrm{X}$ are elements of $\mathrm{D}_{n}$ such that $\mathbf{A}=\mathrm{XBX}^{-1}$, the element IX will similarly transform $\mathbf{B}$ into $\mathbf{A}$. Thus the addition of $\tilde{\mathbf{I}}$ to the group elements does not affect the class structure of those elements not containing $\mathbf{I}$. On the other band $\mathbf{I A}=\mathbf{X}(\mathbf{I B}) \mathbf{X}^{-1}=(\mathbf{I X}) \quad(\mathbf{I B}) \quad(\mathbf{I X})^{-1}$ so the class structure of those elements containing I exactly reproduces that of those elements not containing $\mathbf{I}$.

Let us consider the relations between $D_{3}, D_{3} \times I$ $\left(=\mathrm{D}_{3 d}\right)$ and the hemihedric subgroup $\mathrm{C}_{3 v} . \mathrm{D}_{3}$ contains six elements in three classes as follows: $\mathrm{E} ; \mathrm{C}_{3}, \mathbf{C}_{3}^{2} ; \mathbf{C}_{2}^{\prime}, \mathbf{C}_{3} \mathbf{C}_{2}^{\prime}, \mathbf{C}_{3}^{2} \mathrm{C}_{2}^{\prime}$. $\quad D_{3 d}$ contains these three classes and threemore: $\mathbf{I} ; \mathbf{I C}_{3}, \mathbf{I C}_{3}^{2} ; \mathbf{I C}_{2}^{\prime}, \mathbf{I C}_{3} \mathbf{C}_{2}^{\prime}, \mathbf{I C}_{3}^{2} \mathbf{C}_{2}^{\prime}$. We will now consider which elements must be suppressed to eliminate $\mathbf{I}$ as an element of the group without reverting to $\mathrm{D}_{3}$. Clearly, I must itself be eliminated, as well as the class of $\mathbf{C}_{3} \mathbf{I}$, since $\left(\mathbf{C}_{3} \mathbf{I}\right)^{3}=\mathbf{I}$. If the class of $\mathbf{I C}_{2}^{\prime}$ is eliminated we are back to $D_{3}$, so we retain these three elements, noting that we cannot obtain I from powers of these elements as was done with $\mathbf{I C}_{3}$ since the square of any of them is the unit element. In looking at the classes not containing $\mathbf{I}$, we must retain $\mathbf{E}$ to have a group, and the class of $\mathbf{C}_{3}$ must be retained, since $\mathbf{C}_{3}^{2}=\left(\mathbf{I C}_{2}^{\prime}\right)\left(\mathbf{I C}_{3}^{2} \mathbf{C}_{2}^{\prime}\right)$ and both of the latter are retained. On the other hand, $\mathbf{C}_{2}^{\prime}$ $\left(\mathbf{I C}_{2}^{\prime}\right)=\mathbf{I}$, so we conclude that the class of $\mathbf{C}_{2}^{\prime}$ must also be eliminated, so we obtain for the class structure of $\mathrm{C}_{30}: \mathrm{E} ; \mathrm{C}_{3}, \mathrm{C}_{3}^{2} ; \mathrm{IC}_{2}^{\prime}, \mathbf{I C}_{3} \mathrm{C}_{2}^{\prime}, \mathrm{IC}_{3}^{2} \mathrm{C}_{2}^{\prime}$ which is the same as that of $\mathrm{D}_{3}$ (i.e., the unit element, two elements of order three, and three elements of order two). Furthermore, the multiplicative relations between the elements of $C_{30}$ are the same as those between corresponding elements of $D_{3}$. The product of two elements of $\mathrm{C}_{3}$, neither containing I or both containing I clearly follows the parallel relations in $D_{3}$. If only one contains $\mathbb{I}$, say $\mathbf{I C}_{3} \mathbf{C}_{2}^{\prime}$, its products with the elements $\mathbb{E}, \mathbf{C}_{3}, \mathbf{C}_{3}^{2}$ yield $\mathbb{I}$ times the products of $\mathbf{E}, \mathbf{C}_{3}, \mathbf{C}_{3}^{2}$ with the element $\mathrm{C}_{3} \mathrm{C}_{2}^{\prime}$ of $\mathrm{D}_{3}$. We shall see later the importance of the fact that not only is the class structure the same, but that the corresponding multiplication table is the same (though the latter implies the former) for the two groups.

We may generalize these considerations, and assert that the structure of any hemihedric group is the same as that of the pure rotation group from which it is derived through the intermediary holohedric group of double order. Such groups are said to be isomorphic. The only difference, mathematically superficial, is in the geometric designation of the operations involved. The primary significance of this for the present is that the number of actually independent groups to be considered is considerably less than the 27 finite groups enumerated in table 3 , and that only the structures of the pure rotation groups and the holohedric groups have to be considered.

\subsection{Noncubic Double Groups}

We now turn our attention to the double groups, which we shall designate as ${ }_{1 \mathrm{I}} \mathrm{C}_{30},{ }_{\mathrm{II}} \mathrm{D}_{6 h}$, etc., depending on the corresponding subgroup of $D_{1} \times \mathrm{I}$. This notation will be used when it is desired to particularly emphasize the double group. Usually we shall merely imply these groups through the use of the symbols $\mathrm{C}_{32}, \mathrm{D}_{6 n}$, etc., in connection with half-integral $J$. Some of these groups were first considered by Bethe (1929). The problem was again considered by Opechowski (1940), who clarified many of the ideas involved. We shall begin with the groups ${ }_{11} C_{n}$. The element of $D_{1 / 2}$ for $\phi=2 \pi / n, \theta=\psi=0$ is, from (3.7), $\left[\mathbb{D}_{1 / 2}=\mathrm{U}^{-1}\right]$ the matrix

$$
\left(\begin{array}{ll}
e^{\frac{+i \pi}{n}} & 0 \\
0 & e^{\frac{-i \pi}{n}}
\end{array}\right) .
$$

This is obviously the element of a cyclic group of order $2 n$. We shall call this ${ }_{n 1} \mathrm{C}_{n}$, observing that ${ }_{\mathrm{II}} \mathrm{C}_{n}^{n}$ is given by the matrix $\left(\begin{array}{rr}-1 & 0 \\ 0 & -1\end{array}\right)$ which we usually designate by $\mathbf{R}$. The unit element is then $\mathbf{R}^{2}$. As in the case of $C_{n}$, each element of ${ }_{\mathrm{II}} \mathrm{C}_{n}$ is in a class by itself. The groups $\mathrm{C}_{2 n}$ and ${ }_{\mathrm{II}} \mathrm{C}_{n}$ are isomorphic. The inverse of ${ }_{11} \mathrm{C}_{n}^{k}$ is ${ }_{\mathrm{Ii}} \mathrm{C}_{n}^{2 n-k}=\mathbf{R}_{\mathrm{II}} \mathbf{C}_{n}^{n-k}$.

The double dihedral groups consist of the powers 
of ${ }_{I I} \mathrm{C}_{n}$, and the powers and products of the matrix $\mathrm{D}_{3 / 2}(0, \pi, 0)={ }_{\mathrm{II}} \mathrm{C}_{2}^{\prime}=\left(\begin{array}{rr}0 & +1 \\ -1 & 0\end{array}\right)$ with ${ }_{\mathrm{II}} \mathrm{C}_{n}$ and its powers. The element ${ }_{\text {II }} \mathrm{C}_{2}^{\prime}$ is of order 4 ; its square is $\mathbb{R}$. We note from direct multiplication of the corresponding matrices that $\left({ }_{\mathrm{II}} \mathbb{G}_{2}^{\prime}\right)\left({ }_{\mathrm{II}} \mathrm{C}_{n}\right)=$ $\left({ }_{\mathrm{II}} \mathrm{C}_{n}^{-1}\right)\left({ }_{\mathrm{II}} \mathrm{C}_{2}^{\prime}\right)$. Groups such as this are called dicyclic groups, and have the defining relations as abstract groups $\mathbf{A}^{n}=\mathbb{B}^{2}=\mathbf{R}, \mathbb{R}^{2}=\mathbb{E}, \mathbf{A}^{-1} \mathbf{B}=\mathbf{B A}$, a group of order $4 n$. The class structure may be determined from the matrices themselves, or from the defining relations of the abstract group. A pictorial argument is here inapplicable ab initio, but we shall investigate the relations between the structure of $\mathrm{D}_{n}$ and ${ }_{\mathrm{II}} \mathrm{D}_{n}$. We shall use the defining relations.

The elements which commute with A clearly include the $2 n$ powers of $\mathbf{A}$, but no element involving $\mathbb{B}$ will commute with $\mathbf{A}$ (unless $\mathbf{A}^{-1}=\mathbf{A}$, which implies that $\mathbf{A}=\mathbb{R}$ and that $n=1$, which is excluded, since $n \geq 2$ ). Thus the order of the normalizer of $\mathbf{A}$ is $2 n$, its index is two, and there are two elements in the class of $\mathbf{A}$, which we see immediately from the defining relations are $\mathbf{A}$ and $\mathbf{A}^{-1}$. Generally, $\mathbf{A}^{k}$ and $\AA^{-k}$ will be in the same class, except when $k=n$, since $\mathbf{A}^{n}=\mathbf{R}$ commutes with all elements and is in a class by itself.

The normalizer of $B$ consists of $\mathbb{B}, \mathbf{R}, \mathbb{B}^{3}$, and $\mathrm{E}$, of order 4 and index $n$, so there are $n$ elements in the class of $\mathbb{B}$, obtained by transforming $\mathbb{B}$ with the first $n$ powers of $\mathrm{A}$. We have $\mathrm{ABA}^{-1}=$ $\mathbb{A}^{2} \mathbf{B}$ :, $\mathbf{A}^{2} \mathbf{B A}^{-2}=\mathbf{A}^{4} \mathbf{B}$; etc., or generally the even powers of $\mathbf{A}$, times $\mathbf{B}$. We have left over the class of $\mathrm{AB}$, with normalizer $\mathrm{AB}, \mathbb{R},(\mathrm{AB})^{3}, \mathbb{E}$. The $n$ elements in the class of $A B$ may likewise be obtained by transforming by the first $n$ powers of $\mathbb{A}$, yielding generally the odd powers of $\mathbf{A}$ times $\mathbb{B}$. Summarizing, there is the class of $\mathbf{E}$ with one element, the class of $\mathbb{R}$ with one element, the $(n-1)$ classes of $\mathbf{A}^{k}$ with two elements, the class of $\mathbb{B}$ with $n$ elements, and the class of $\mathbb{A B}$ with $n$ elements. Altogether there are $n+3$ classes. We notice that the class structure is the same for both odd and even $n$.

It will now be interesting to compare the class structure of ${ }_{\text {II }} \mathrm{D}_{n}$ with that of the group $\mathrm{D}_{n}$ from which it is derived. Let us write the defining relations of $D_{n}$ in terms of the abstract elements $A$ and $B$ as $A^{n}=B^{2}=\mathbb{E} ; A B=B A^{-1}$, so that we distinguish corresponding elements by italics. Let us further, in ${ }_{{ }_{1}} \mathrm{D}_{n}$, write powers of $\mathbb{A}$ greater than $n$ in terms of products of powers of A less than $n$ and the element $\mathbf{R}$. Thus, $\mathbf{A}^{n+k}=$ $\mathbb{R A}^{k}$, and $\mathbf{A}^{-k}=\mathbf{A}^{2 n-k}=\mathbb{R A}^{n-k}$. Let us consider the class of $A$, which is $\left(A, A^{-1}\right)$. There are four elements in ${ }_{\mathrm{II}} \mathrm{D}_{n}$ corresponding to these two, $\mathbf{A}$ and $\mathbf{R A}$ to the first, $\mathbf{A}^{-1}=\mathbf{R A}{ }^{n-1}$ and $\mathbf{A}^{n-1}$ to the second. These four elements are in separate classes by pairs $\left(\mathbf{A}, \mathbb{R A}^{n-1}\right)$ and $\left(\mathbb{R A}, \mathbf{A}^{n-1}\right)$. If $n$ is even, these are both classes of order $2 n$. If $n$ is odd, A is of order $2 n$, but $\mathbf{R A}$ is of order $n$. Similarly, the class of $A^{k}, A^{n-k}$ will generally split into $\left(\mathbf{A}^{k}, \mathbf{R} \mathbf{A}^{n-k}\right)$ and $\left(\mathbf{A}^{n-k}, \mathbf{R} \mathbf{A}^{k}\right)$. If $n$ is even, these are of the same order; if $n$ is odd, these are of the same order for even $k$, and of different order for odd $k$. The sole exception to this will occur if $k=n / 2$ (which will require $n$ to be even), where there is but one element in the class $\left(A^{n / 2}\right)$ and the two elements $\mathbf{A}^{n / 2}, \mathbf{R A}^{n / 2}$ are in the same class.

Let us now consider the classes of $\boldsymbol{B}$ and $\mathbb{B}$. If $n$ is odd, the class of $\mathbf{B}$ contains $\mathbf{A}^{2} \mathbf{B}, \mathbf{A}^{\mathbf{A}} \mathbf{B}$, $\ldots \mathbf{A}^{n-1} \mathbf{B}, \mathbb{R A B}, \mathbb{R A}^{3} \mathbf{B} \ldots \mathbb{R A}^{n-2} \mathbf{B}$, but it does not contain $\mathbb{R B}$, nor does it contain $\mathbf{A B}$. The class of $\mathbf{A B}$ also contains $\mathbf{A}^{3} \mathbf{B}, \ldots \mathbf{A}^{n-2} \mathbf{B}$, $\mathbb{R B}, \mathbb{R A}^{2} \mathbf{B} \ldots \mathbb{R A}^{n-1} \mathbf{B}$. On the other hand, the class of $B$ contains $B, A B, A^{2} B, \ldots A^{n-1} B$, so the class of $B$ will split into two classes, one containing $\mathbf{B}$ and $\mathbf{R A B}$, the other containing $\mathbf{A B}$ and RB (and other elements as indicated above). If $n$ is even the class of $\mathbf{B}$ will still include only the even powers of $\mathbf{A}$ times $\mathbf{B}$, but $\mathbf{A}^{n}=\mathbf{R}$ will now be included, so $\mathbf{B}$ and $\mathbb{R B}$ are now in the same class. The class of $\boldsymbol{B}$ now involves only $\boldsymbol{B}$ and the even powers of $\boldsymbol{A}$ times $\boldsymbol{B}$, so for $n$ even the class of $\boldsymbol{B}$ will not split, but will merely contain twice as many elements.

We may summarize this behavior by the following diagram, where we give in one column the typical classes of $\mathrm{D}_{n}$, in the next column the corresponding classes of ${ }_{\text {II }} \mathrm{D}_{n}$, for both odd and even $n$.

\begin{tabular}{|c|c|}
\hline \multicolumn{2}{|r|}{$n$ odd } \\
\hline $\mathrm{D}_{n}$ & ${ }_{\mathrm{II}} \mathrm{D}_{n}$ \\
\hline$E$ & $\begin{array}{l}(\boldsymbol{E}) \\
(\boldsymbol{R})\end{array}$ \\
\hline$\left(A^{k}, A^{n-k}\right)$ & $\begin{array}{l}\left(\mathbf{A}^{k}, \mathbf{R} \mathbf{A}^{n-k}\right) \\
\left(\mathbf{A}^{n-k}, \mathbf{R} \mathbf{A}^{k}\right)\end{array}$ \\
\hline$\left(B, A B, A^{2} B \ldots\right)$ & $\begin{array}{l}\left(\mathbf{B}, \mathbf{R A B}, \mathbf{A}^{2} \mathbf{B} \ldots\right) \\
\left(\mathbf{R B}, \mathbf{A B}, \mathbf{R A}^{2} \mathbf{B} .\right)\end{array}$ \\
\hline \multicolumn{2}{|r|}{$n$ even } \\
\hline$(\boldsymbol{E})$ & $\begin{array}{l}(\mathbf{E}) \\
(\mathbf{R})\end{array}$ \\
\hline$\left(A^{k}, A^{n-k}\right)$ & $\begin{array}{l}\left(\mathbf{A}^{k}, \mathbb{R A}^{n-k}\right) \\
\left(\mathbf{A}^{n-k_{0}}, \mathbf{R A}^{k}\right)\end{array}$ \\
\hline$\left(A^{n / 2}\right)$ & $\left(\mathbf{A}^{n / 2}, \mathbf{R} \mathbf{A}^{n / 2}\right)$ \\
\hline$\left(B, A^{2} B, A^{4} B \ldots\right)$ & $\left(\mathbf{B}, \mathbb{R B}, \mathbf{A}^{2} \mathbb{B}, \mathbb{R} \mathbf{A}^{2} \mathbb{B} \ldots\right)$ \\
\hline$\left(A B, A^{3} B, A^{5} B.\right)$ & $\left(\mathbf{A B}, \mathbf{R A B}, \mathbf{A}^{3} \mathbf{B}, \mathbf{R} \mathbf{A}^{3} \mathbf{B}, \ldots\right)$ \\
\hline
\end{tabular}

We see that the class structure of ${ }_{\text {II }} D_{n}$ follows the same pattern for both odd and even $n$; the pattern varies in the case of $\mathrm{D}_{n}$, in particular, for classes of elements of order 2. Results of the comparison may be summarized in to the following rules, first given by Opechowski:

1. For each class of $D_{n}$ of order other than 2, there are two classes of ${ }_{I I} D_{n}$ each having the same number of elements as the class of $\mathrm{D}_{n}$.

2. If there is but one class in $D_{n}$ of order 2 ( $n$ is odd), this will in ${ }_{\mathrm{II}} \mathrm{D}_{n}$ follow rule (1).

3 . If there are two or more classes in $D_{n}$ of order $2,(n$ is even) i.e., there are two or more mutually perpendicular two-fold axes, these classes 
will not split in ${ }_{\text {II }} \mathrm{D}_{n}$ but will contain twice as many elements. These rules also hold for the cubic groups, essentially special cases of $D_{2}$ or $D_{4}$. Finally, we may observe that we deduced the existence of $n+3$ classes in ${ }_{\text {II }} \mathrm{D}_{n}$. 'This should be considered in the light of the above rules with the $(n+3) / 2$ classes of $D_{n}(n$ odd), all of which split in ${ }_{\text {II }} \mathrm{D}_{n}$ or $3+n / 2$ classes for $n$ even, where $n / 2$ classes split in ${ }_{\text {II }} \mathrm{D}_{n}$.

The relations between the double pure rotation groups and the associated holohedric and hemihedric groups is the same as between the single rotation groups and their associated holohedric and hemihedric groups, and therefore need not be discussed separately.

\subsection{Reps and Characters}

We shall now examine briefly the notions of an invariant subgroup and of a factor group. We shall see that these, together with the orthogonality relation for group characters, will permit us to derive the characters for most of the finite groups in which we shall be interested. Thile these group characters have been derived by very general considerations, a detailed study will prove to be very instructive. Let us consider a subgroup $\mathrm{S}$ of $\mathrm{G}$, of order $s$ and index $n(n s=g)$. If $\mathbf{X}$ is any element of $\mathrm{G}, \mathrm{S}$ is an inrariant subgroup if $\mathrm{XSX}^{-1}=\mathrm{S}$, or $\mathrm{XS}=\mathrm{SX}$. This does not imply that every element $S_{i}$ of $S$ commutes with $\mathbf{X}$, but only that $\mathbf{X} \mathbf{S}_{i}=\mathbf{S}_{j} \mathbf{X}$, or that the set of elements $\mathbf{X S}$ is the same as the set SX, differing only in the order if at all. If $n=2$, we may expand $G$ into the sum $S+S T$, where $T$ is an element of $G$ not in $\mathrm{S}$, or into the sum $\mathrm{S}+\mathrm{TS}$. We see that $\mathrm{ST}=\mathrm{TS}$, or that any subgroup of index 2 is an invariant subgroup. It is clear that $S$ is either a class of $G$ or a sum of classes.

Let us associate with each element $\mathbf{X}$ of $\mathrm{G}$ the complex SX. While there are $g \mathbf{X}$ 's, there are only $n$ distinct complexes SX. Let $\mathbf{P}$ and $\mathbf{Q}$ be elements of $\mathrm{G}$ with product $\mathbf{P Q}=\mathbf{R}$. The associated complexes, similarly multiplied, gire (SP) $(\mathrm{SQ})=\mathrm{SSPQ}=\mathrm{S}(\mathrm{PQ})=(\mathrm{SR})$, since $\mathrm{S}$ commutes with any group element, and $S S=S$. Te thus see that a relation $\mathrm{PQ}=\mathrm{R}$ holds either for the group elements themselves or for the associated complexes SP, SQ, and SR. These $n$ complexes constitute a factor group of order $n$, the unit element being $S$. The importance of the factor group for our purposes lies in the fact that any rep of the factor group will likewise be a rep of the original group. Since the factor groups are of lower order, their reps may frequently be found by inspection.

As an illustration of these ideas, let us consider the group $\mathrm{D}_{2}$ with elements $\mathrm{E}, \mathbf{C}_{2}, \mathrm{C}_{2}^{\prime}, \mathbf{C}_{2} \mathbf{C}_{2}^{\prime}$. Since $\mathrm{C}_{2}, \mathrm{C}_{2}^{\prime}$, and $\mathrm{C}_{2} \mathrm{C}_{2}^{\prime}$ are all of order $2, \mathrm{E}$ and any one of them will constitute a subgroup of index 2 , and hence an invariant subgroup. There are thus three "proper" factor groups (of order $>1$ and $<4)$ :
$1:\left(\mathrm{E}, \mathrm{C}_{2}\right)\left(\mathrm{C}_{2}^{\prime}, \mathrm{C}_{2} \mathrm{C}_{2}^{\prime}\right)$
2: $\left(\mathrm{E}, \mathrm{C}_{2}^{\prime}\right)\left(\mathrm{C}_{2}, \mathrm{C}_{2} \mathrm{C}_{2}^{\prime}\right)$ 3: $\left(\mathrm{E}, \mathrm{C}_{2} \mathrm{C}_{2}^{\prime}\right)\left(\mathrm{C}_{2}, \mathrm{C}_{2}^{\prime}\right)$.

We observe that the set of number's 1,1 and $1,-1$ constitute reps for the factor groups. We may then obtain four reps for the whole group by associating with each of the three factor groups the two reps. This would generally yield six reps, but those arising from the rep 1,1 are the same for all three factor groups. These reps are tabulated below. Those numbered 1-3 are obtained from the corresponding factor group by the association 1, -1 of the factor group, while (4) arises from 1,1 .

$\begin{array}{lrrrr} & \mathrm{E} & \mathrm{C}_{2} & \mathrm{C}_{2}^{\prime} & \mathrm{C}_{2} \mathrm{C}_{2}^{\prime} \\ (1) & 1 & 1 & -1 & -1 \\ (2) & 1 & -1 & 1 & -1 \\ (3) & 1 & -1 & -1 & 1 \\ (4) & 1 & 1 & 1 & 1\end{array}$

We are now prepared to discuss the characters of the reps of specific groups, to tabulate their numerical values, and to systematize the description of states in terms of their behavior under the group operations, just as the quantum numbers $L, S$, and $J$, and $M_{L}, M_{S}$, and $M_{J}$ describe the behavior of states under general rotations. In the original work of Bethe (1929), the various reps were designated generally by $\Gamma$, and distinguished by numerical subscripts such as $\Gamma_{3}, \Gamma_{5}$, etc. This notation has persisted in this field, though it has no systematic meaning and does not suggest the actual behavior of the state under consideration. An alternate notation has been highly developed in connection with the theory of groups as applied to molecular vibrations (Herzberg, 1945; Mulliken, 1955 ), and it is a modification of this that we shall employ. While we shall discuss the notation applicable to each group as it is discussed in detail below, it seems desirable to cover the notation generally at this time, particularly to point out variations from the notation appropriate to molecular' spectra.

We shall consider first the single groups, i.e. those appropriate to integral $J$. We shall find that the reps are of dimensionality three, two, or one. Those of dimensionality three, which arise only in the cubic groups, are designated by the symbol $T$. If the inversion is a group element, the symbol will carry the subscript " $g$ " or " $u$ ", as appropriate, and, if necessary, they may be further distinguished, essentially by convention, by subscripts " 1 " or " 2 ". "Two-dimensional reps will be designated as $E$, again with a " $g$ " or " $u$ " if appropriate. The two-dimensional reps of $\mathrm{D}_{\infty}$ and $\mathrm{C}_{\infty}$, infinite in number, will be found to correspond to the pair of states $\pm M_{J}$ of the free ion; they will thus be distinguished by $M_{J}$ appended as a subscript. For these two groups, molecular spectroscopists use the symbols $\Pi, \Delta$, $\Phi$, etc. It is believed that the notation of the present work is more adaptable to crystal spectra, since $D_{\infty} h$ and $C_{\infty v}$ are only useful approximations to a case of lower symmetry, where the " $E$ " notation is also used in molecular spectroscopy.

One-dimensional reps have as elements numbers, real or complex, of magnitude 1 . If all elements 
are real, i.e., +1 or -1 , they are designated as " $A$ " if the element for rotation about the z-axis is +1 , " $B$ " if the element is -1 . 'They may be further distinguished by subscripts 1,2 according as the element for rotation about the $y$-axis is +1 or $-1 ; g$ or $u$ for the inversion; or $\left({ }^{\prime}\right)$ or $\left({ }^{\prime \prime}\right)$ for $+1,-1$, respectively, for reflection in the $x-y$ plane. While this is the general policy to which the molecular notation conforms, it is usually abandoned in the case of the group $\mathrm{D}_{2}$ or $\mathrm{D}_{2 h}$. This is because the $x, y$, and $z$ axes are distinguishable only by convention, and the reps are called $B_{1}, \mathcal{B}_{2}, \mathcal{B}_{3}$. This does not seem to be warranted; even the assignment of subscripts in the $B$ 's must be done by convention, so one may as well conform to the general rule. If the elements are not all real, we use the symbol $C_{k}$, according to the quantity $(\sqrt[n]{1})^{k}$. The general procedures of molecular spectroscopy merge a complex rep with its conjugate, which must also appear, as a two-dimensional rep designated as " $E$ ". We are interested in distinguishing these reps because of their separation when a magnetic field is applied.

The double groups do not appear in problems of molecular vibrations. Their reps are of dimensionality four, two, and one. That one of dimensionality four is the appropriate collection of the elements of the rep $D_{3 / 2}$ of $\mathfrak{l}_{2}$, so we designate it as $D_{3 / 2}$ here also. Some of those of dimensionality two which appear are similarly collections of elements of $D_{1 / 2}$, which we continue to designate as $D_{1 / 2}$; those two dimensional reps not so designated are assigned the symbol ${ }_{2} S_{k}$, where "S", represents the idea of "spin" or "specific", the anterior subscript for a two-dimensional rep, and the posterior one (if necessary) by convention. Similarly, the one-dimensional reps are designated " $S$ ", with a distinguishing subscript according to the root of 1 involved in the rep.

The reps of the cyclic groups $C_{n}$ are all onedimensional. It is clear that just as the group consists of the $n$ powers of $\mathrm{C}_{n}$ so also will the $n$ powers of $\sqrt[n]{1}$ serve as a rep. It is similarly clear that the $n$ powers of $(\sqrt[n]{1})^{k}$ will also serve as a rep, where $k$ may be $2,3,4, \ldots n$. If $k=n$, we designate it by $A$, a general notation for a rep where every rotation about the $z$-axis is the unit one-dimensional matrix. If $n$ is even, for $k=n / 2$ we use $B$, similarly a general notation for a rep where the element of the rep corresponding to the basic group element $\mathrm{C}_{n}$ is the $1 \times 1$ matrix $(-1)$. In other words, $A$ is a symmetric rep, $B$ an antisymmetric rep. Note that $C_{n-k}$ is the complex conjugate rep to $C_{k}$.

The double cyclic groups ${ }_{\mathrm{II}} \mathrm{C}_{n}$ are isomorphous with the group $\mathrm{C}_{2 n}$, and hence the reps are the various powers of $(\sqrt[2 n]{1})^{k}$. For $k$ even, we designate the rep by $C_{k / 2}$ or $A$ or $B$ since it will then be also the corresponding rep of $\mathrm{C}_{n}$. For $k$ odd, we shall call the rep $S_{k}$ ( $S$ for spin or specific) to emphasize that it is peculiar to the double group. $S_{k}$ and $S_{2 n-k}$ are complex conjugate reps. We note that, if $\sqrt[3]{1}=\omega, \omega^{2}, 1$ then $\sqrt[6]{1}=-\omega^{2}, \sqrt[12]{1}=-i \omega$.
The dihedral groups are less easily analyzed. The reps (and characters) for $\mathrm{D}_{2}$ were obtained above in connection with the illustration of the application of the factor group concept. In terms of numbering there used, we shall describe (4) as $A_{1}$, (1) as $A_{2}$, (2) as $B_{1}$, and (3) as $B_{2}$. Here again, $A$ is used for reps which are totally symmetric for rotations about the $z$-axis, being distinguished by subscripts according to the behavior for rotations about the secondary $y$-axis. $B$ is used where the basic rotation about the $z$-axis is antisymmetric, and again distinguishing various behavior for rotations about the $y$-axis.

The group $\mathrm{D}_{3}$ contains the invariant subgroup $\left(\mathrm{E}, \mathrm{C}_{3}, \mathrm{C}_{3}^{2}\right)$, so two reps are obtained as the rep $(1,1)$ and $(1,-1)$ of the factor group. Since (3.11) admits of the solution $2^{2}+1^{2}+1^{2}=6$, the other rep is two-dimensional and its character may be deduced from the orthogonality relation for group characters. The one dimensional reps are designated as $A_{1}$ and $A_{2}$, while two-dimensional reps generally are designated as $E$.

For $\mathrm{D}_{4}, 8$ elements in 5 classes, eq (3.11) admits the solution $2^{2}+1^{2}+1^{2}+1^{2}+1^{2}=8$, so there are four 1-dimensional reps and one 2-dimensional rep. Since $\left(\mathbb{E}, \mathbb{C}_{4}, \mathbf{C}_{4}^{2}, \mathbb{C}_{4}^{3}\right)$ is an invariant subgroup, two reps are again the reps of the factor group $(1,1)$ and $(1,-1)$, designated $A_{1}$ and $A_{2}$. We may also verify that $\left(\mathbb{E}, \mathrm{C}_{4}^{2}\right)$ is an invariant subgroup, whose factor group is isomorphous with $\mathrm{D}_{2}$, having the structure $\left(\mathrm{E}, \mathrm{C}_{4}^{2}\right)\left(\mathrm{C}_{4}, \mathrm{C}_{4}^{3}\right)\left(\mathrm{C}_{2}^{\prime}, \mathrm{C}_{4}^{2} \mathrm{C}_{2}^{\prime}\right)$ $\left(\mathrm{C}_{4} \mathrm{G}_{2}^{\prime}, \mathbf{C}_{4}^{3} \mathrm{C}_{2}^{\prime}\right)$. $A_{1}$ and $A_{2}$ of $\mathrm{D}_{2}$ give no new reps, but $B_{1}$ and $B_{2}$ of $\mathrm{D}_{2}$ will give us new reps, which we shall designate as $B_{1}$ and $B_{2}$ in this case also. The two-dimensional rep $E$ may now be found from the orthogonality condition.

In the case of $D_{6}, 12$ elements in 6 classes, $2^{2}+2^{2}+1^{2}+1^{2}+1^{2}+1^{2}=12$. The group $C_{6}$ is an invariant subgroup, and the $(1,1)$ and $(1,-1)$ of its factor group $\mathrm{C}_{2}$ are $A_{1}$ and $A_{2}$ respectively of $\mathrm{D}_{6}$. The group $\mathrm{C}_{3}$ is also an invariant subgroup, with factor group $\mathrm{D}_{2}$, whose reps $B_{1}$ and $B_{2}$ give $B_{1}$ and $B_{2}$ of $\mathrm{D}_{6}$. Finally, the group $\left(\mathbf{E}, \mathrm{C}_{2}\right)$ is an invariant subgroup with factor group $\mathrm{D}_{3}$, whose rep $E$ will yield a new rep $E_{2}$ of $D_{6}$. We find from the orthogonality condition the rep $E_{1}$. The reps are numbered according to the reps of $\mathrm{D}_{\infty} h$ with which they may be correlated (table 10).

For the double groups generally, cyclic or dicyclic, $(\mathbb{E}, \mathbb{R})$ is an invariant subgroup whose factor group is the corresponding single group, so the reps of the single group are also reps of the double group, as we have already seen in the case of the cyclic gioups. For any half-integral $J$, the traces of $\mathbf{D}_{J}(\mathbb{E})$ and $\mathbf{D}_{J}(\mathbf{R})$ are $(2 J+1)$ and $-(2 J+1)$, respectively. Hence we see that we cannot resolve a faithful representation for half-integral $J$ into a sum of reps, any one of which has the same trace for $\mathbf{E}$ and $\mathbf{R}$. This latter is clearly the case for those reps derived from the invariant subgroup $(\mathbb{E}, \mathbf{R})$, namely those which are also reps of the single group, so we conclude that they will not be useful for describing 
states arising from an odd number of electrons. Those reps which are peculiar to the double groups we shall call specific reps (the basis for the " $S$ " notation in the cyclic groups used earlier); we include the non-specific reps for the completeness required by the orthogonality theorem and for the treatment of selection rules.

${ }_{\mathrm{II}} \mathrm{D}_{2}$ contains 8 elements in 5 classes, so $2^{2}+1^{2}+1^{2}+1^{2}+1^{2}=8$. The four 1-dimensional reps are the reps of $D_{2}$, so the only specific rep is two-dimensional. It is obvious that this must be equivalent to the appropriate elements of $D_{1 / 2}$, or in other words, the representation is its own rep. Te shall emphasize this by designating the rep as $D_{1 / 2}$.

${ }_{\text {II }} \mathrm{D}_{3}$ has 12 elements in 6 classes, whence $2^{2}+2^{2}+1^{2}+1^{2}+1^{2}+1^{2}=12$. Half of these are the reps of $D_{3}$, so there are two specific one dimensional reps and one specific two-dimensional rep. We observe that $\left(\mathrm{E},{ }_{\mathrm{II}} \mathbf{C}_{3}^{2}, \mathbf{R}_{\mathrm{II}} \mathbf{C}_{3}\right)$ is an inrariant subgroup. Its associated complexes (cosets) are $\left({ }_{\mathrm{II}} \mathrm{C}_{2}^{\prime},{ }_{\mathrm{II}} \mathrm{C}_{3 \mathrm{II}}^{2} \mathrm{C}_{2}^{\prime}, \mathbf{R}_{\mathrm{II}} \mathrm{C}_{3 \mathrm{II}} \mathrm{C}_{2}^{\prime}\right),\left({ }_{\mathrm{II}} \mathrm{C}_{3}, \mathbf{R}\right.$, $\mathbf{R}_{\mathrm{II}} \mathbf{G}_{3}^{2}$ ) and ( ${ }_{\mathrm{II}} \mathrm{C}_{3 \mathrm{II}} \mathbf{C}_{2}^{\prime}, \mathbf{R}_{\mathrm{II}} \mathbf{C}_{2}^{\prime}, \mathbf{R}_{\mathrm{II}} \mathbf{C}_{3 \mathrm{II}}^{2} \mathrm{C}_{2}^{\prime}$ ) yielding a factor group isomorphous with $\mathrm{C}_{4}$. The reps $A$ and $B$ of $C_{4}$ yield no new reps, but $C_{1}$ and $C_{3}$ will yield specific reps which we shall distinguish as $S_{1}$ and $S_{3}$. Because of these two 1-dimensional reps into which the representation from $D_{1 / 2}$ could perhaps split, we cannot conclude that the representation from $D_{1 / 2}$ is also a rep here, but this proves to be actually the case if the two-dimensional character is calculated from the orthogonality relations. As before, we call this rep $D_{1 / 2}$.

${ }_{\mathrm{I} 1} \mathrm{D}_{4}$ has 16 elements in 7 classes. Since $2^{2}+2^{2}+2^{2}+1^{2}+1^{2}+1^{2}+1^{2}=16$, we find on subtracting the reps of $\mathrm{D}_{4}$ that there are but two specific reps, both two-dimensional. One of these must be $D_{1 / 2}$; the other, derived from the orthogonality relations, we shall call ${ }_{2} S$.

${ }_{\text {II }} \mathrm{D}_{6}$ has 24 elements in 9 classes. Equation (3.11) gives us $2^{2}+2^{2}+2^{2}+2^{2}+2^{2}+1^{2}+1^{2}+1^{2}+$ $1^{2}=24$. Eliminating the reps of $D_{6}$, we have three specific reps, all two-dimensional. One of these must be $D_{1 / 2}$. Unfortunately, we cannot obtain either of the other two characters by the methods used so far, and an alternate procedure must be used. We obtain from table 7 the character of the representation arising from $D_{3 / 2}$, which is four-dimensional and hence reducible. By application of the expansion theorem (3.10) we see that $D_{1 / 3}$ is included once in the representation. The other rep involved we shall call ${ }_{2} S_{1}$. The final rep may now be obtained from the orthogonality theorem as ${ }_{2} S_{2}$.

The holohedric groups, single or double, are defined as the direct product of the group $(\mathbf{E}, \mathbf{I})=\mathrm{I}$ with a pure rotation group. The reps may be obtained as products of the reps $(1,1)$ and $(1,-1)$ of I with those of the pure rotation group. The reps are designated like those of the pure rotation group, with the additional subscript " $g$ " if $(1,1)$ is used and " $u$ " if $(1,-1)$ is used.

It has already been pointed out that the hemi- hedric groups are isomorphous with the corresponding (in the sense of table 3 ) pure rotation group and hence have the same reps. Most of these hemihedric reps are described just like the corresponding pure rotation reps. The exceptions are the groups $\mathrm{D}_{3 h}, \mathrm{C}_{3 h}, \mathrm{C}_{s}$, and $\mathrm{S}_{4}$. For the first three of these, the reps are classified according to behavior under the operation $\mathbf{I C}_{2}=\boldsymbol{\sigma}_{h}$, symmetric reps carrying a (') while antisymmetric reps have ("). $\quad \mathrm{D}_{3 h}$ is further subclassified according to $\mathrm{G}_{2}^{\prime}$. This difference in notation is peculiar to the single groups-the specific reps of all hemihedric double groups will be designated like those of the corresponding pure rotation group. In the case of $\mathrm{S}_{4}$, isomorphic with $\mathrm{C}_{4}$, the generating element is taken as the operation $\mathbf{I C}_{4}^{3}=\mathbf{C}_{4} \mathbf{I C}_{2}=\mathbf{C}_{4} \boldsymbol{\sigma}_{h}$, which is usually considered as the fundamental rotation-reflection operation. This is why the rep $C_{1}$ of $\mathrm{S}_{4}$ corresponds to $C_{3}$ of $\mathrm{C}_{4}$ rather than to $C_{1}$ of $\mathrm{C}_{4}$.

\subsection{Infinite and Cubic Groups}

We have finally to discuss the infinite groups involving infinitesimal rotations about the $z$-axis, namely $\mathrm{C}_{\infty}, \mathrm{C}_{\infty h}, \mathrm{D}_{\infty}, \mathrm{D}_{\infty h}, \mathrm{C}_{\infty t}$, and the cubic groups. In $\mathrm{C}_{\infty}$, the group operation consists of arbitrary rotations $\mathrm{G}(\phi)$ such that $\mathrm{G}\left(\phi_{1}\right) \mathrm{G}\left(\phi_{2}\right)=$ $\mathrm{C}\left(\phi_{2}\right) \quad \mathrm{G}\left(\phi_{1}\right)=\mathrm{G}\left(\phi_{1}+\phi_{2}\right)$, and that $\mathrm{C}(2 \pi)=\mathrm{C}(0)$. If $k$ is any number, $e^{i k_{\phi}}$ will meet the first requirement, but the second limits $k$ to a real integer, positive or negative, including zero. If $k=0$, we shall call the rep $A$, otherwise $C_{k}$. For the group ${ }_{\text {II }} \mathrm{C}_{\infty}$, the first of the above conditions holds, but the second is replaced by $\mathbf{G}(2 \pi)=-\mathbf{C}(0)$. Here $k$ must be half-integral, positive or negative, and we designate the reps by $S_{2 k}$ ( $2 k$ is an odd integer). $\mathrm{C}_{\infty h}$ is $\mathrm{C}_{\infty} \times \mathrm{I}$, so the reps for this group will carry the subscript " $g$ " or " $u$ ".

In $\mathrm{D}_{\infty}$, the group elements consist of the rotations $\mathrm{C}(\phi)$ and the rotation $\mathrm{C}_{2}^{\prime}$, such that $\mathrm{C}_{2}^{\prime} \mathrm{G}(\phi)=$ $\mathrm{G}(-\phi) \mathbf{C}_{2}^{\prime}$. Considering $\mathrm{C}_{\infty}$ as an invariant subgroup, the reps $(1,1)$ and $(1,-1)$ of the factor group $\mathrm{C}_{2}$ yield reps $A_{1}$ and $A_{2}$ of $\mathrm{D}_{\infty}$ : As discussed under $\mathrm{C}_{\infty}, e^{i k \phi}$ will serve as a rep insofar as $\mathrm{C}(\phi)$ is concerned, but the operation $\mathrm{C}_{2}^{\prime}$ makes $e^{i k \phi}$ and $e^{i k(-\phi)}$ equivalent. Except when $k=0$ (which gives $A_{1}$ and $A_{2}$ ) our rep for $\mathrm{C}(\phi)$ must have the twodimensional form

$$
\left(\begin{array}{ll}
e^{i k \phi} & 0 \\
0 & e^{-i k \phi}
\end{array}\right)
$$

and $\mathrm{C}_{2}^{\prime}$ will assume the form

$$
\left(\begin{array}{rr}
0 & -1 \\
-1 & 0
\end{array}\right)
$$

which will transform $\mathrm{C}(\phi)$ into $\mathrm{C}(-\phi)$. It may be shown that there are no other reps. These reps we shall call $E_{k}$, where $k$ is a positive integer. In the case of ${ }_{\text {II }} \mathrm{D}_{\infty}$, the reps become

$$
{ }_{\mathrm{II}} \mathrm{C}(\phi)=\left(\begin{array}{ll}
e^{i k \phi / 2} & 0 \\
0 & e^{-i k \phi / 2}
\end{array}\right), \quad{ }_{\mathrm{II}} \mathrm{G}_{2}^{\prime}=\left(\begin{array}{cc}
0 & 1 \\
-1 & 0
\end{array}\right)
$$


and we shall designate them as ${ }_{2} S_{k}(k$ odd). Reps of $\mathrm{D}_{\infty h}$ will have the additional subscripts " $g$ " or " $u$ ", while reps of the hemihedric group $C_{\infty}$ will be designated like those of $\mathrm{D}_{\infty}$.

In the group $\mathrm{O}$, there are 24 elements in 5 classes. These are the class of $\mathbf{E}, 1$ element; the class of $\mathbf{C}_{4}, 6$ elements; the class of $\mathbf{C}_{2}, 3$ elements; the class of $\mathbf{C}_{3}^{\prime}$, the body diagonal axes, 8 elements; and the class of $\mathbf{C}_{2}^{\prime}$ (here, $\mathbf{C}_{2}^{\prime}$ is not along the $y$-axis), 6 elements. (3.11) gives $3^{2}+3^{2}+2^{2}+1^{2}+1^{2}=24$. The set of elements $\left(\mathbb{E}, 3 \mathbf{C}_{2}, 8 \mathbf{C}_{3}^{\prime}\right)$ is an invariant subgroup with factor group of order 2 . Its reps $(1,1)$ and $(1,-1)$ are the reps $A_{1}$ and $A_{2}$ of $\mathrm{O}$. If we consider $D_{J}$ for $J=1$, we find that neither $A_{1}$ nor $A_{2}$ is in the representation. Since it cannot be reduced to $A_{1}+E$ or $A_{2}+E$ ( $E$ being the twodimensional rep), it must itself be one of the threedimensional reps. These are generally designated as $T$-this particular rep we shall call $T_{1} . D_{J}$, $J=2$, contains neither $A_{1}, A_{2}$, or $T_{1}$, so it must reduce to $E+T_{2}$, but we cannot separate them. On going to $J=3$, we find from this seven-dimensional representation, $A_{1}$ and $T_{1}$ accounting for four. The remaining three must then be $T_{2}$ which, subtracted from the $J=2$ case leaves $E$. The groups $\mathrm{O}_{h}$ and $\mathrm{T}_{d}$ follow our previous considerations on pure rotation, holohedric, and hemihedric groups. The reps of $T_{z}$ parallel those of $\mathrm{O}$.

The double group ${ }_{\text {II }} \mathrm{O}$ has 48 elements. The splitting into classes, as compared with the group $O$, follows the same rules as for the dihedral groups given in section 3.7 , so the class structure is: $\mathbb{E}(1) ; \mathbb{R}(1) ;{ }_{1 I} \mathbf{C}_{4}(6) ; \mathbb{R}_{\mathrm{II}} \mathbf{C}_{4}(6) ;{ }_{\mathrm{II}} \mathrm{C}_{2}(6) ;{ }_{\mathrm{II}} \mathrm{G}_{3}^{\prime}$ (8); $\mathbb{R}_{I I} \mathbf{G}_{3}^{\prime}$ (8); ${ }_{\text {II }} \mathbf{C}_{4 I I} \mathbf{C}_{2}^{\prime}$ (12). (3.11), after eliminating the reps of $\mathrm{O}$, indicates three reps for ${ }_{\mathrm{II}} \mathrm{O}$, two 2-dimensional and one 4-dimensional. As usual, $D_{3 / 2}$ is one of these reps. $D_{3 / 2}$ in this case is also a rep, the four-dimensional one. The remaining rep obtained from orthogonality we call ${ }_{2} S$.

The group $\mathrm{T}, 12$ elements, may be separated into the class $\mathbb{E}(1) ; \mathbf{C}_{2}(3) ; \mathbf{C}_{3}(4)$; and $\mathbb{C}_{3}^{2}(4)$. It is interesting to note that, in spite of the relatively high symmetry, a rotation $\mathbb{C}_{3}$ and its inverse $\mathbb{C}_{3}^{-1}=\mathbb{C}_{3}^{2}$ are in different classes. (3.11) yields $3^{2}+1^{2}+1^{2}+1^{2}=12$. The elements $\left(\mathbb{E}, 3 \mathbf{C}_{2}\right)$ constitute an invariant subgroup, with a factor group of order $3, C_{3}$. The reps $A, C_{1}$ and $C_{2}$ of $\mathrm{C}_{3}$ are thus the one-dimensional reps $A, C_{1}$, and $C_{2}$ of $\mathrm{T}$. The rep $T$ may then be obtained from orthogonality and completeness considerations. The group ${ }_{\text {II }} \mathrm{T}, 24$ elements divides (section 3.7) into $\mathrm{E}(1) ; \mathbf{R}(1) ;{ }_{\mathrm{II}} \mathbf{C}_{2}(6) ;{ }_{\mathrm{II}} \mathrm{G}_{3}$ (4); $\mathbf{R}_{\mathrm{II}} \mathbf{C}_{3}$ (4); ${ }_{\text {II }} \mathrm{G}_{3}^{2}$ (4); and $\mathrm{R}_{\mathrm{II}} \mathrm{C}_{3}^{2}$ (4). Subtracting off the reps of $\mathrm{T}$, we have $2^{2}+2^{2}+2^{2}=12$, indicating reps we shall call $D_{3 / 2},{ }_{2} S_{1}$, and ${ }_{2} S_{2}$. As the notation suggests, $D_{3 / 2}$ is its own rep. Unfortunately, our previous techniques for finding reps fail in this case, since the reps ${ }_{2} S_{1}$ and ${ }_{2} S_{2}$ always appear together and cannot be separated. We must therefore proceed to a more general method.

The product of any two classes of a group $\left[\mathrm{C}_{a}\right]$ and $\left[\mathrm{C}_{b}\right]$ with $N_{a}$ and $N_{b}$ elements, respectively, will be a total of $N_{a} N_{b}$ elements, not necessarily all distinct, which may themselves be written as a sum of classes $\left[\mathbf{G}_{q}\right]$, or $\left[\mathbf{C}_{a}\right]\left[\mathbf{C}_{b}\right]=\sum_{q} N_{a, b_{i} q}\left[\mathbf{C}_{q}\right]$, with $N_{a, b ; q}$ a small nonnegative integer. A particular $q$ may not appear at all, or may appear more than once. It may be shown (Murnaghan, 1938) that this expansion implies the following relation between the traces of the $i$-th rep:

$$
N_{a} N_{b} \chi_{i a} \chi_{i b}=\chi_{i E} \sum_{q} N_{a, b ; q} N_{q} \chi_{i q}
$$

where $\chi_{i E}$ is the trace of the unit element, i.e., the dimension of the rep. By developing in detail the multiplication table of a group, sufficient relations may be found to calculate the complete character table of any group. In the present case, we already have all but two of the seven possibilities for ${ }_{\text {II }} \mathrm{T}$. A solution of these equations for $\chi_{i E}=2$ will then give the desired characters.

\subsection{Table of Group Characters}

The foregoing considerations have been summarized in table 8, a table of group characters for both the single and double groups in which we shall be interested. Each portion of the table is constructed around one of the single holohedric groups. Typical elements of the various classes of the group, together with an indication of the total number of elements in the class, are shown to the right of the group designation, while the designations of the several reps are shown under the group designation. For convenience, we also give the notation of Bethe (1929). The characters for the various classes and reps are the entries in the table. The classes, reps, and characters for the corresponding pure rotation group may be obtained by dropping all classes containing $I$ and the subscripts " $g$ " and " $u$ " on the reps.

Above and to the left of each basic character table are shown the classes and reps of the corresponding hemihedric groups. The appropriate group designations are given upward and to the left of that of the basic holohedric group, while the class structure is given along the same row and the several reps in the same column, so that both classes and reps correspond to those of the holohedric group. A careful study of these will indicate very clearly the relations between a bolohedric group and its hemihedric derivatives.

The characters and reps for the specific reps of the double groups are given in table 8 immediately below the reps of the single groups. Because of the frequent splitting of the classes of the single group into two classes discussed above, there will in such cases be two characters for each class of the single group. If $\mathbf{A}$ is a class element of the single group, the first double group character will be that for the class of ${ }_{\text {II }} \mathbf{A}$, the second for that of $\mathbf{R}_{\text {II }} \mathbf{A}$. Upon inspection, it will be seen that such pairs of characters have opposite signs, while those arising from classes of the single group which do not split are all zero. 


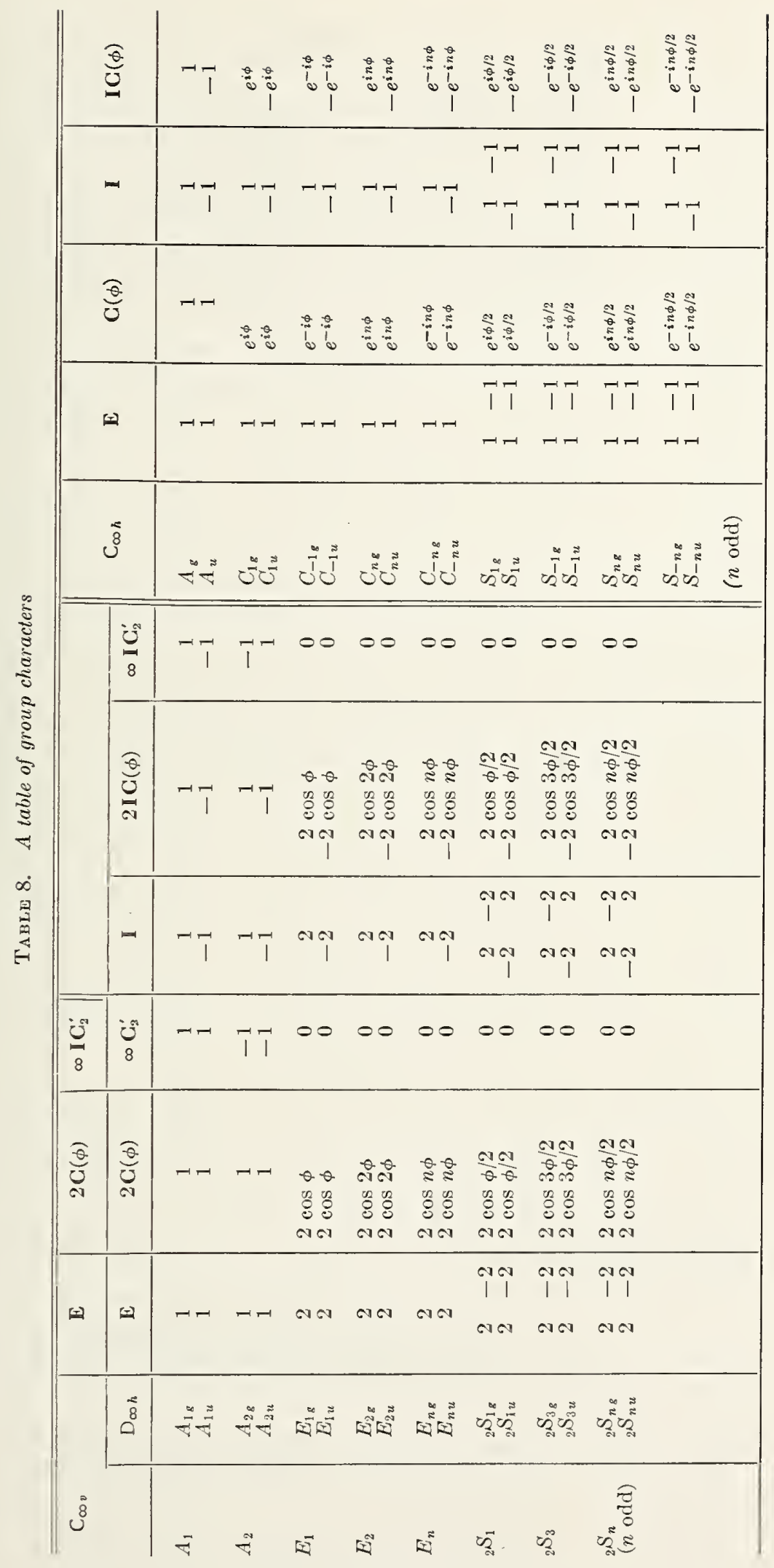




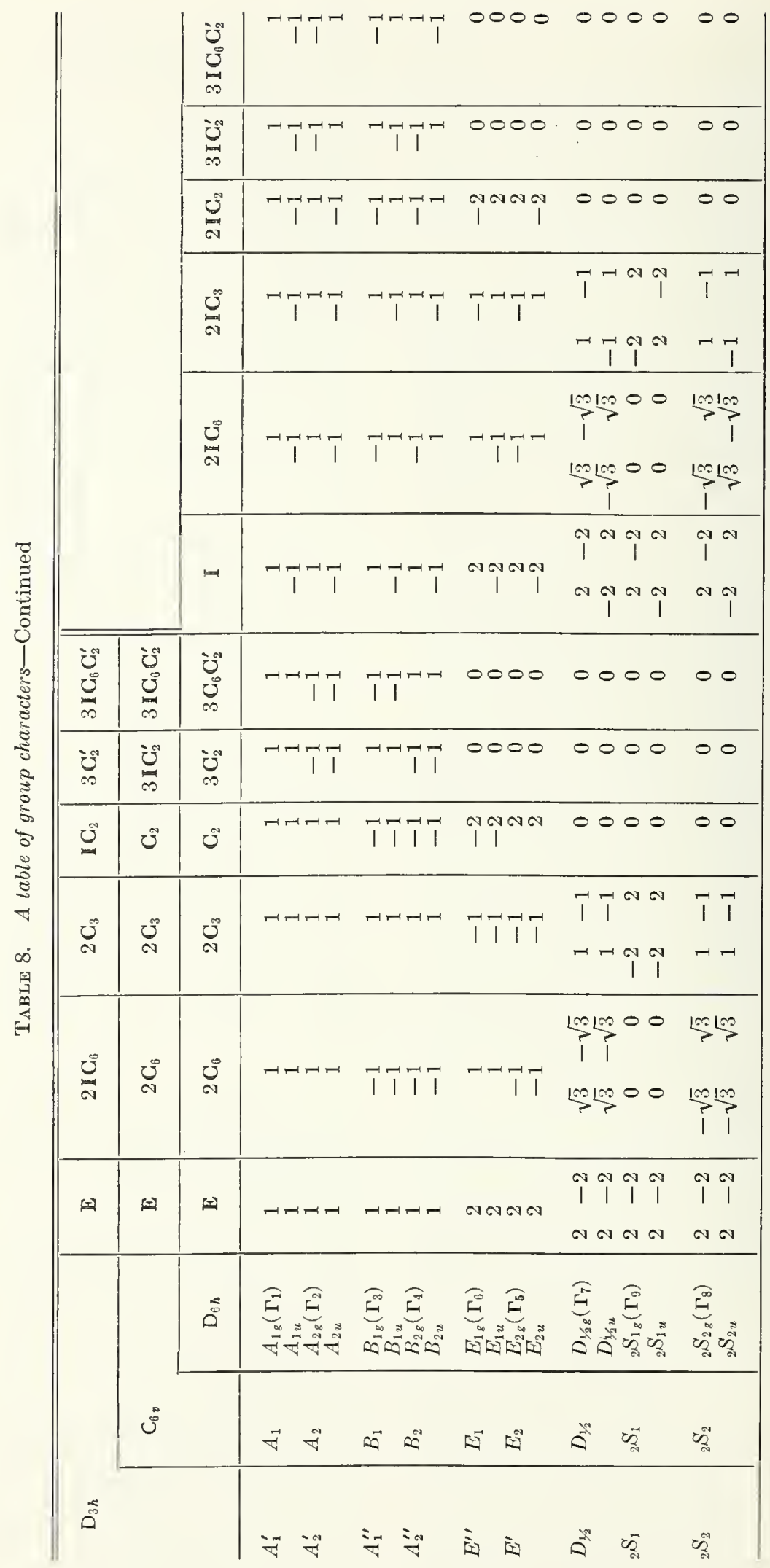




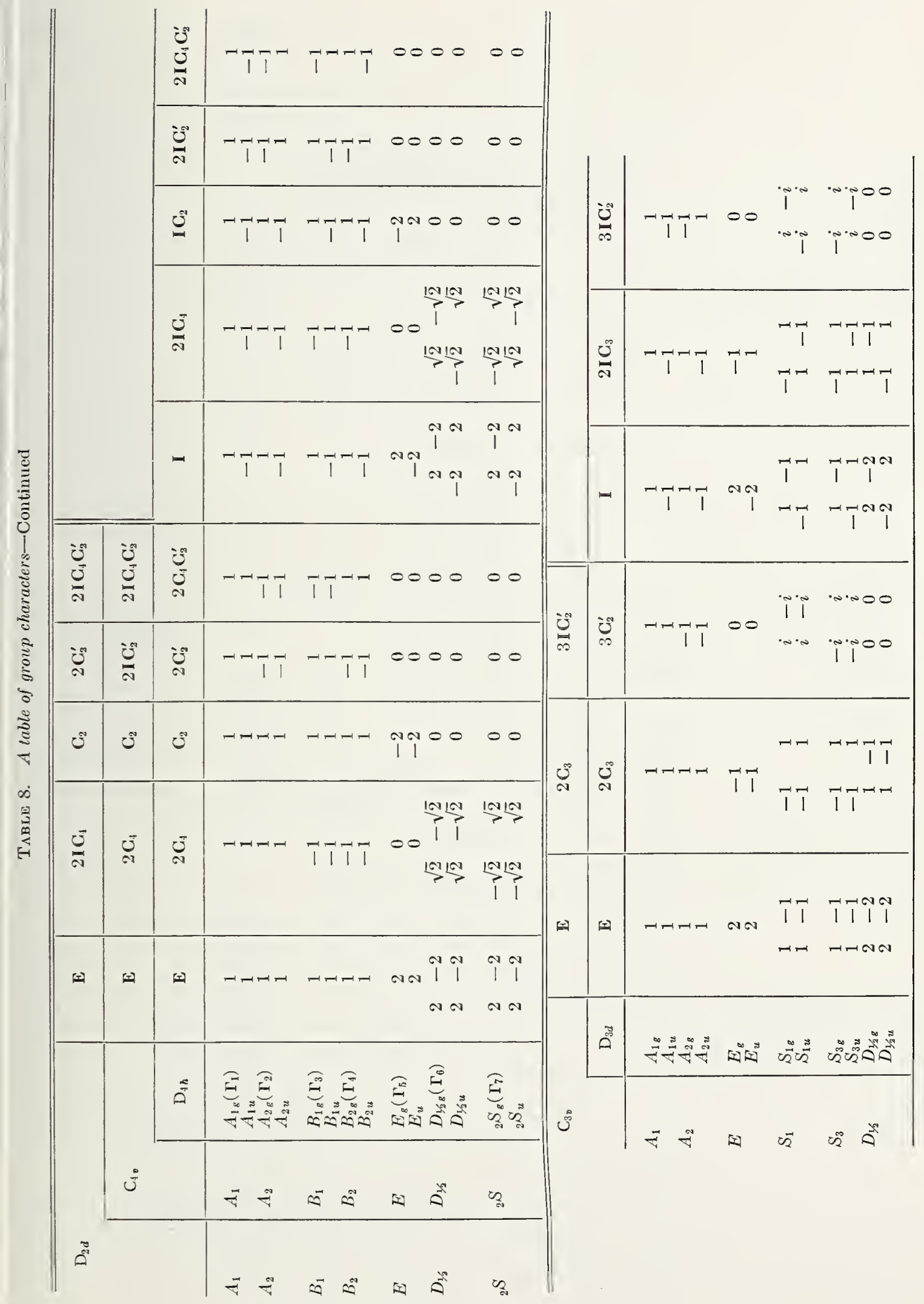




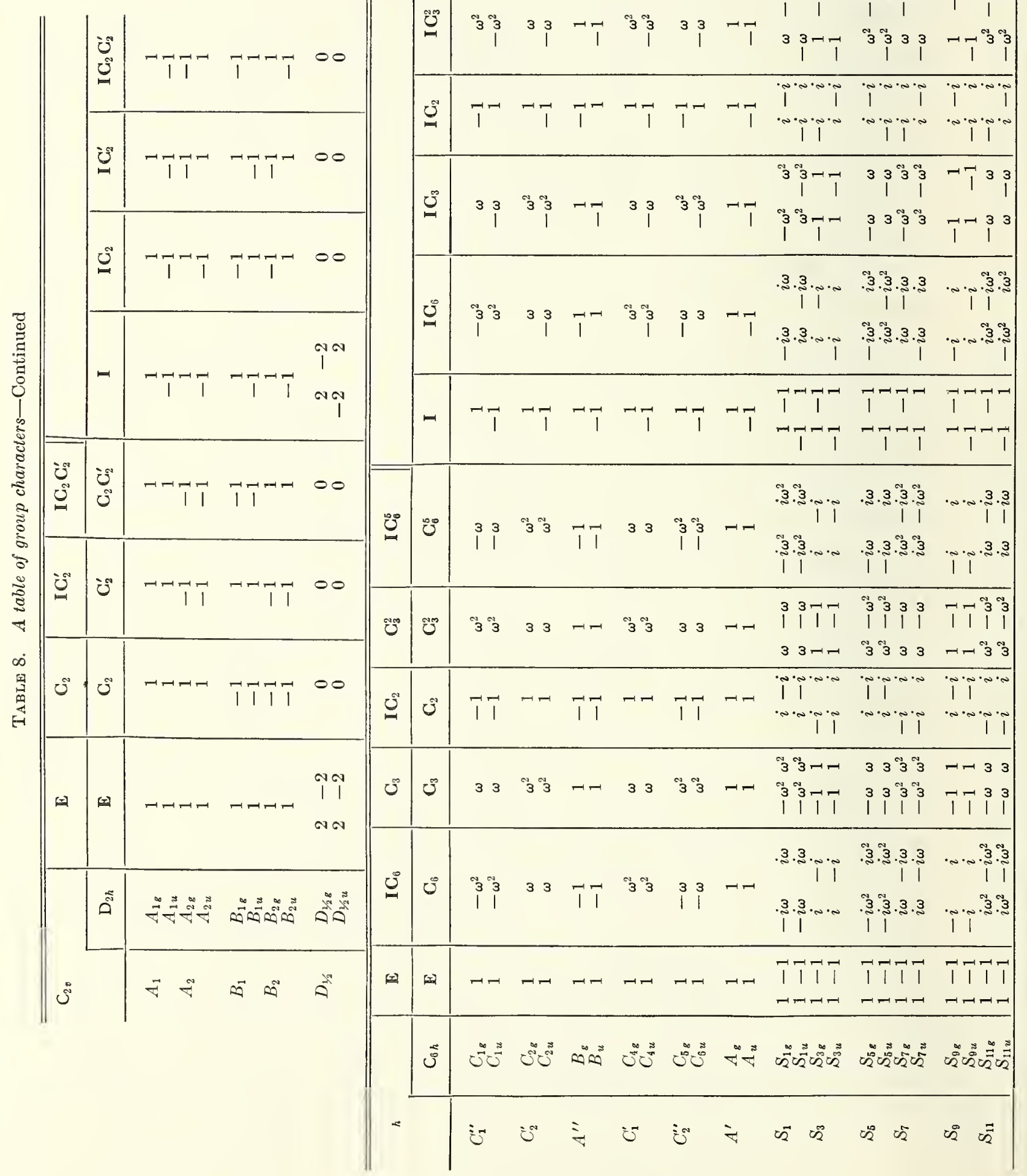




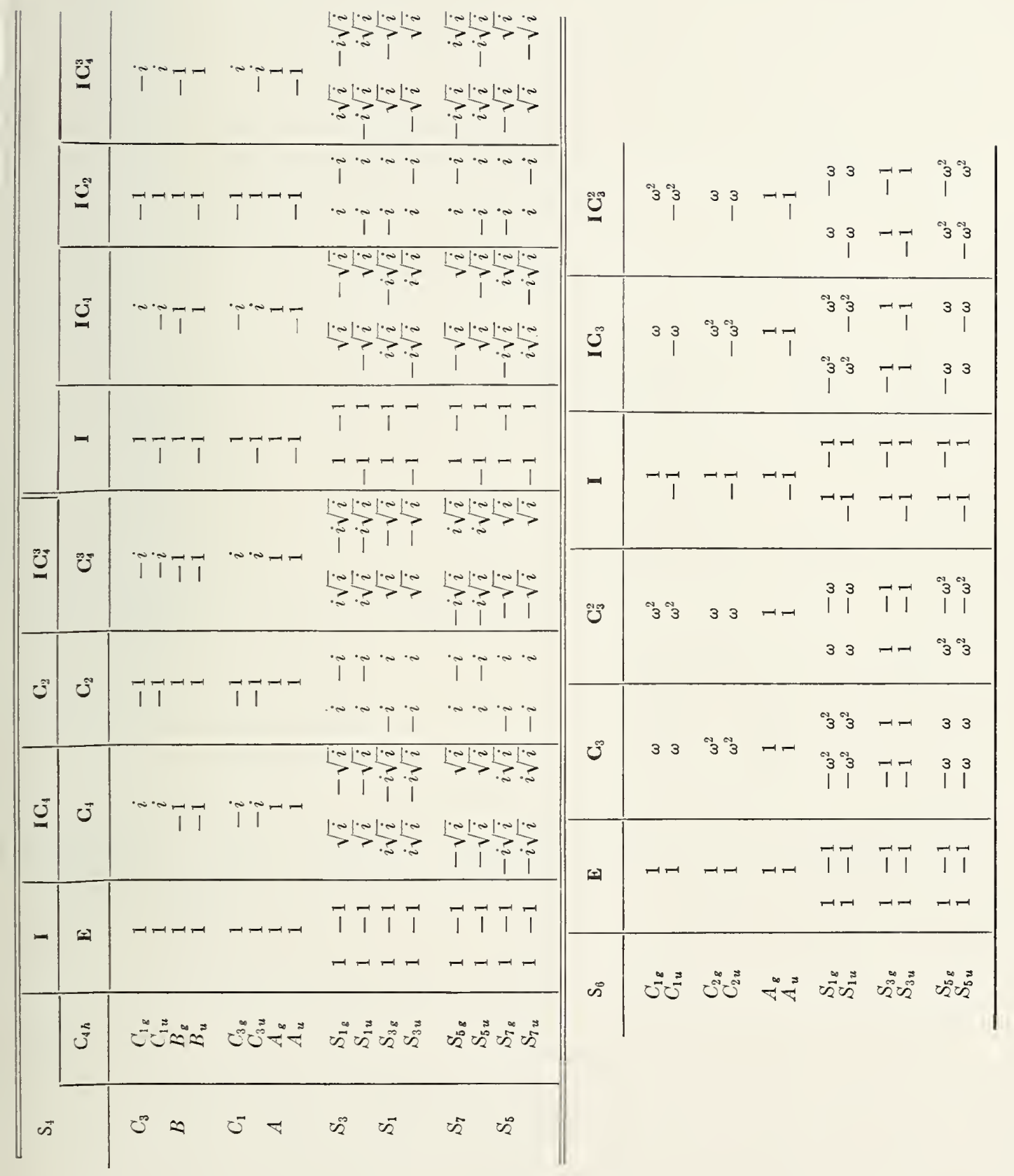


TABLE 8. A table of group characters-Continued

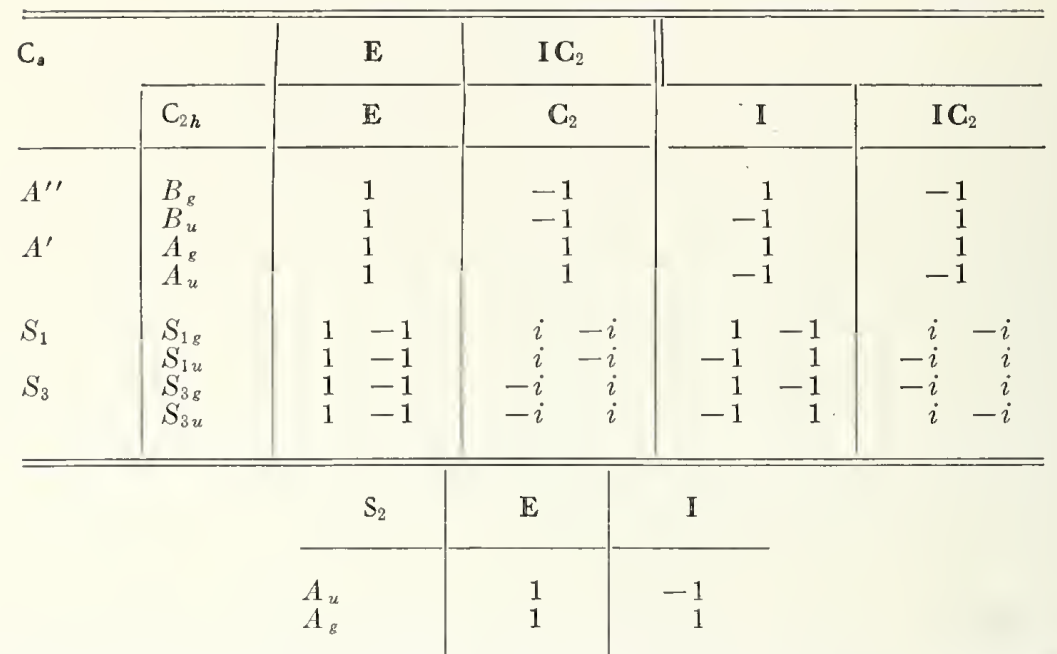

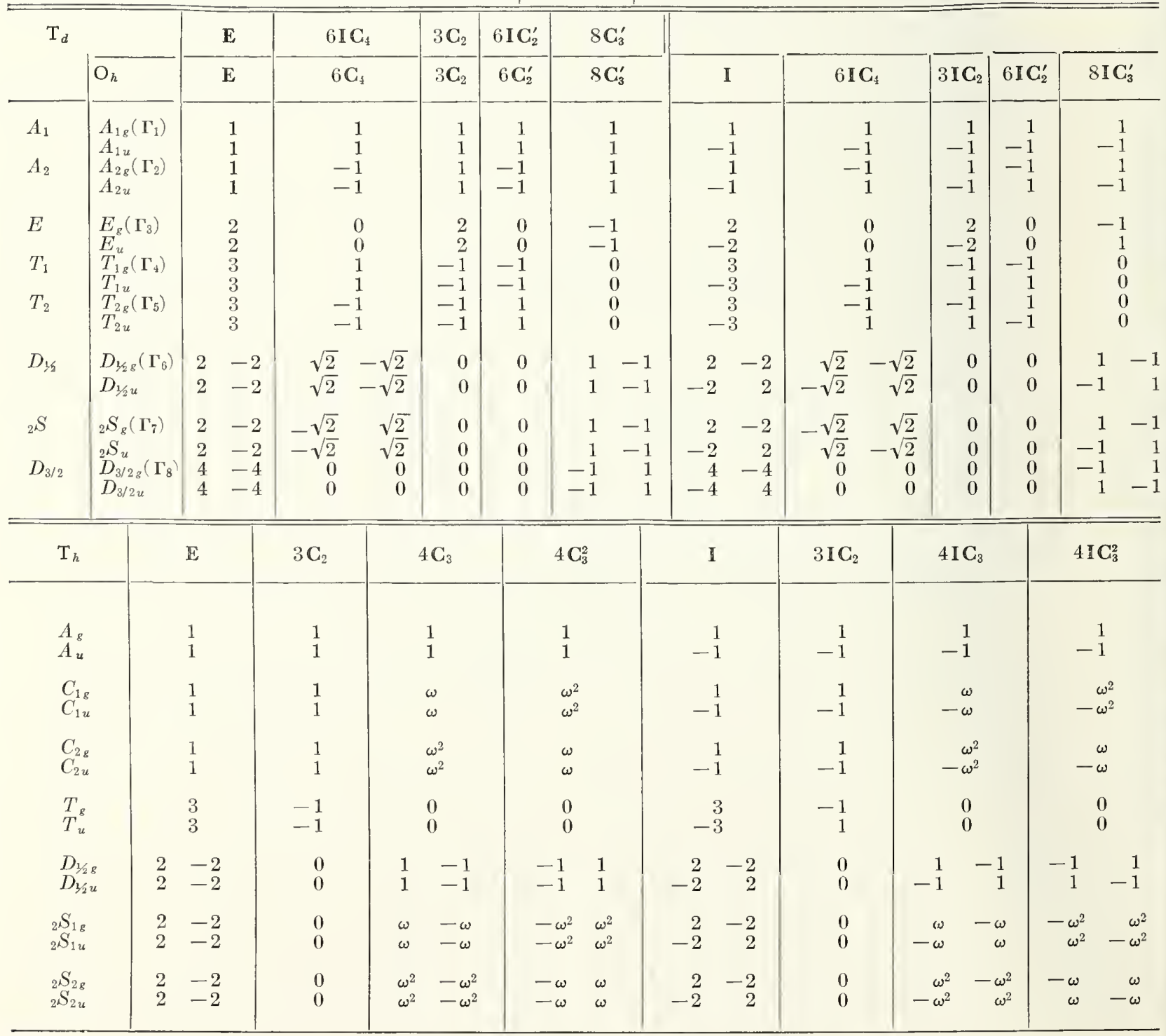




\section{Degeneracy, Selection Rules, and Polarization}

\subsection{Splitting of Free Ion Levels}

We are now in a position to apply the results of our previous considerations to the problem of degeneracy in the presence of a perturbing external field. The general procedure has been covered in the previous section; we consider here the specific example of a free ion level described as ${ }_{4 u}\left(J=4,(-1)^{\left.\Sigma l_{i}=-1\right)}\right.$ in a field of $\mathrm{C}_{6 v}$ symmetry. From table 8 we obtain the class structure of $\mathrm{C}_{60}$ as $\mathrm{E}, 2 \mathrm{C}_{6}, 2 \mathrm{C}_{3}, \mathrm{C}_{2}, 3 \mathbf{I C}_{2}^{\prime}, 3 \mathbf{I C}_{3} \mathbf{C}_{2}^{\prime}$. The traces of the matrices of the rotation group $J=4$ corresponding to the first four of these classes may be obtained directly from table 7 . Those for the last two classes will be -1 times the traces of the corresponding pure rotation. We obtain the following for the character table of a reducible representation of $\mathrm{C}_{60}$. Te also include for immediate convenience the reps $A_{1}$ and $A_{2}$.

\begin{tabular}{r|r|r|r|r|r|r}
\hline \hline & $\mathrm{E}$ & $2 \mathrm{C}_{6}$ & $2 \mathrm{C}_{3}$ & $\mathrm{C}_{2}$ & $3 \mathrm{I} \mathrm{C}_{2}^{\prime}$ & $3 \mathrm{IC}_{3} \mathrm{C}_{2}^{\prime}$ \\
\hline & 9 & -2 & 0 & 1 & -1 & -1 \\
\hline $4 a$ & 1 & 1 & 1 & 1 & 1 & 1 \\
$A_{1}$ & 1 & 1 & 1 & 1 & -1 & -1 \\
\hline$A_{2}$ & & & & & \\
\hline
\end{tabular}

The expansion theorem for group characters (3.10) was given in terms of the weighted traces

and

$$
\xi_{i j}=\sqrt{\frac{N_{j}}{g}} \chi_{i j}
$$

$$
\Xi_{j}=\sqrt{\frac{N_{j}}{g}} \mathrm{X}_{j}
$$

but it is usually more convenient to use the traces directly. Thus $\boldsymbol{a}_{i}=\boldsymbol{\xi}_{i} \cdot \boldsymbol{\Xi}(3.10)$ becomes $g a_{i}$ $=\sum_{j} N_{j} \chi_{i}^{*} \mathrm{X}_{j}$. Using this latter form, we obtain for $A_{1}: 12 a_{A_{1}}=1(1)(9)+2(1)(-2)+2(1)(0)+1(1)(1)$ $+3(1)(-1)+3(1)(-1)=0$, so $A_{1}$ will not appear in the reduction. Likewise, $12 a_{A_{2}}=1(1)(9)$ $+2(1)(-2)+2(1)(0)+1(1)(1)+3(-1)(-1)$ $+3(-1)(-1)=12$, so $A_{2}$ will appear in the reduction once.

The results of this and similar calculation for all the subgroups of $D_{J}$ of present interest are given in table 9 . As in table 8 , the results for the pure rotation groups are not given separately, and may be obtained from the associated holohedric group by omitting the " $g$ "- " $u$ " distinction. Only the group I has been omitted; this has no symmetry apart from the inversion, and states are either $A_{g}$ or $A_{u}$. The integral range of $J$ is from 0 to 8 , while half-integral $J$ ranges from $1 / 2$ to $15 / 2$. If only electric fields constitute the perturbation on the free ion, the appropriate group may be obtained from table 5 or from crystallographic considera- tions. For a pure magnetic field, we shall consider only a uniform field with symmetry $C_{\infty h}$. In case both fields are present, the appropriate group is the group of those symmetry operations common to the electric and magnetic fields. Unless the magnetic field is parallel to one of the symmetry axes of the crystal field, there will in general be no symmetry common to both perturbations.

In accordance with our previous discussions, the physical implication of this reduction is that all one dimensional reps appearing in the reduction represent states arising from the free ion level $J$, which are not related by symmetry to any of the other states so arising. 'These states, then, are either nondegenerate or "accidentally" degenerate, the latter being due to numerical values of the parameters hence very improbable in any actual case. Any two-dimensional rep represents a pair of states from the original $2 J+1$ which are essentially degenerate, being related by symmetry operations, but not degenerate with the other $2 J-1$ states. Similarly for any reps of higher dimension. If a particular rep appears only once in the reduction the state or states it represents may be uniquely associated with a particular combination of the original $2 J+1$ states. If the rep appears more than once there will be two or more states of the original $2 J+1$ having the same symmetry properties, and the appropriate states in the crystal may be a linear combination of these. This can only be determined from the numerical parameters involved. These considerations are modified by Kramers degeneracy to be considered in detail in section 4.2. Certain pairs of reps are noted in table 9 as being Kramers conjugate states. These states, even though belonging to different reps, are degenerate if the perturbing field is purely electrostatic.

It is often of considerable interest to follow the behavior of a given level during the introduction of perturbing fields of increasingly lower symmetry. For example, one may wish to see the effect on the levels under $\mathrm{D}_{3 d}$ symmetry of a reduction of symmetry to $\mathrm{C}_{3 v}$. This may be accomplished by the correlation table, table 10 . Since most of the groups with which we are presently concerned are subgroups of $\mathrm{D}_{\infty h}$, we start with the reps of $\mathrm{D}_{\infty h}$. For each group of lower symmetry, the designation of the level or levels is given in the appropriate column. Again, the pure rotation groups are not given separately, requiring only the suppression of " $g$ " " $u$ " in the associated holohedric group. The procedure involved here is the application to the characters of the reps of $\mathrm{D}_{\infty}$ precisely the same procedures as were applied to the rotationinversion group characters in constructing Table 9. Notice that here, in many cases, the rep of $\mathrm{D}_{\infty n}$ is still a rep of the subgroup, since the levels frequently remain twofold degenerate.

For an example, let us consider the state $\mathscr{2}_{u}$ under the influence of a crystal field of symmetry 
TABLE 9. Type and number of perturbed levels

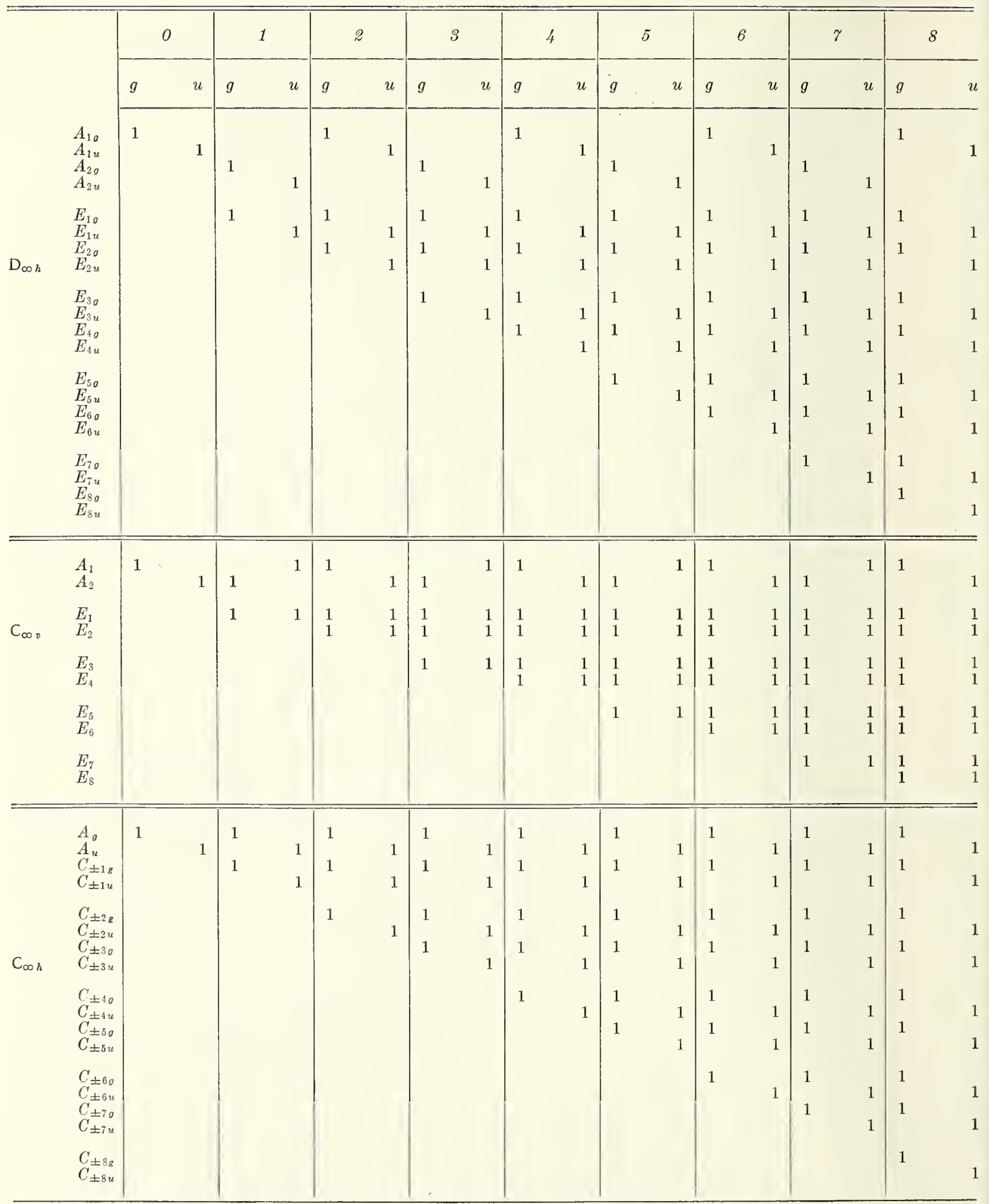

Note: The states $C_{n g}, C_{-n g}$, and $C_{n u}, C_{-n u}$, are Kramers conjugate states. 


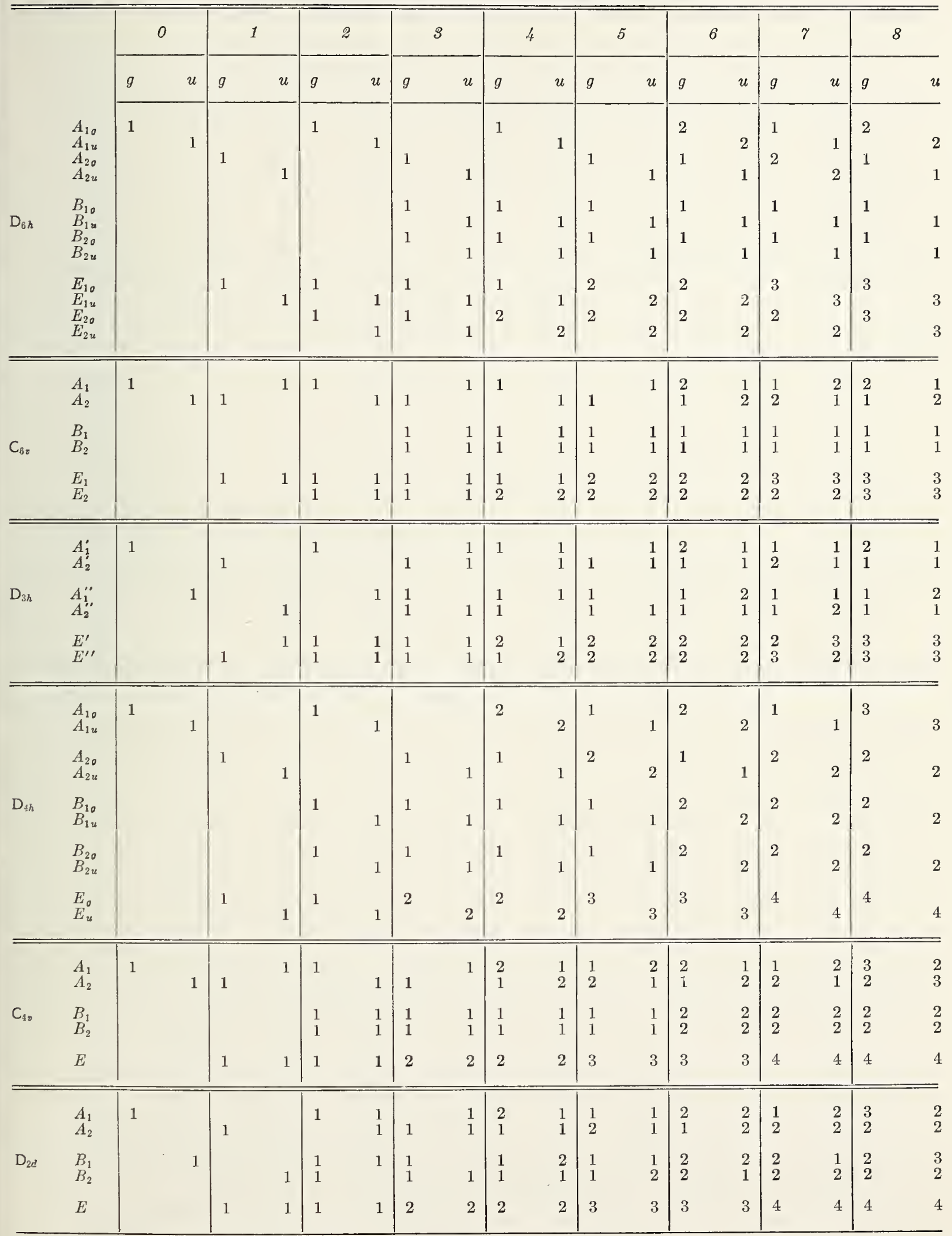


TABLE 9. Type and number of perturbed levels-Continued

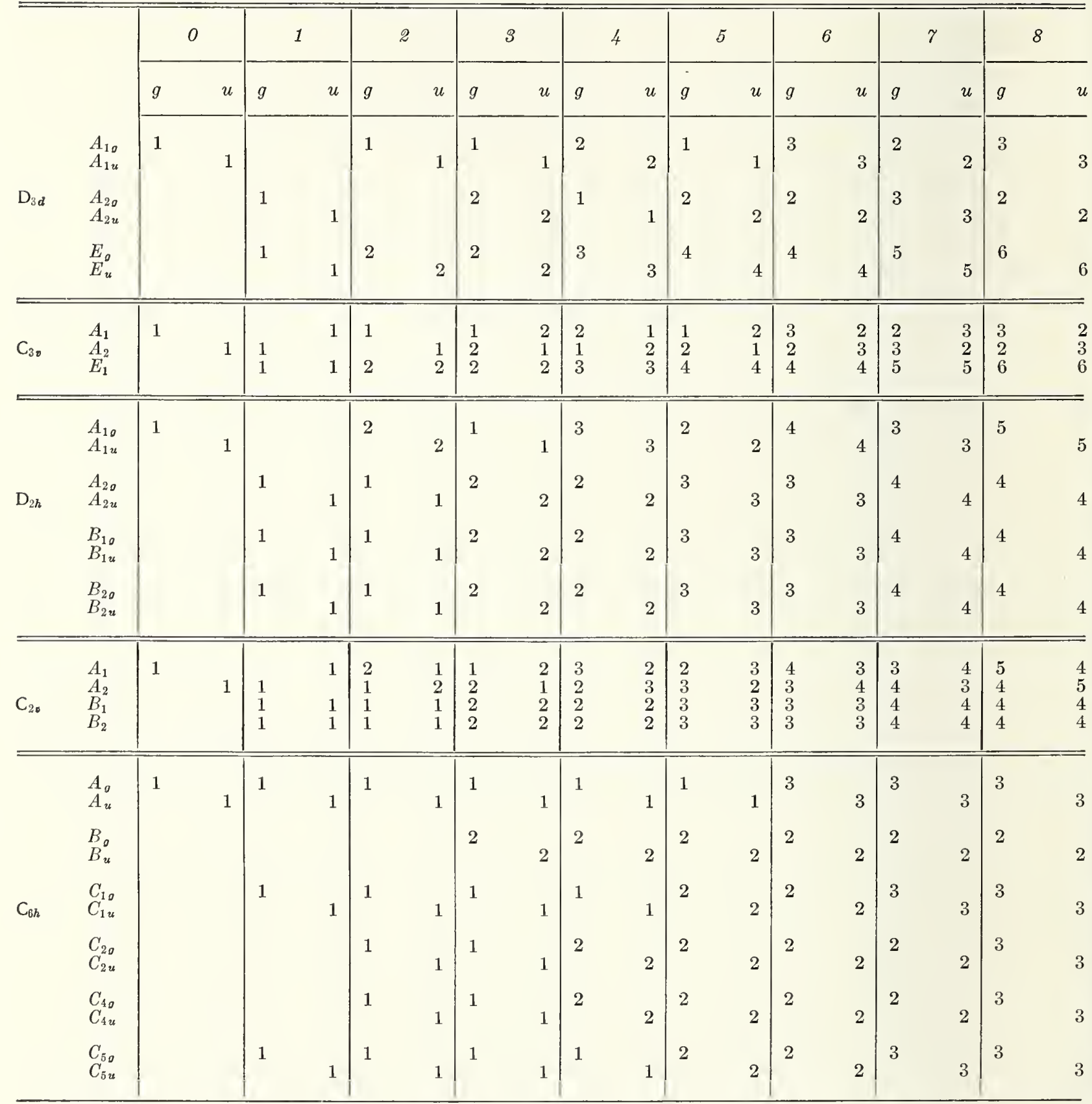

Note: The states $\left(C_{1 \sigma}, C_{5 q}\right),\left(C_{1 u}, C_{5 u}\right),\left(C_{2 \sigma}, C_{4 \vartheta}\right),\left(C_{2 u}, C_{4 u}\right)$ are Kramers conjugate states.

\begin{tabular}{|c|c|c|c|c|c|c|c|c|c|c|c|c|c|c|c|c|c|c|}
\hline \multirow{3}{*}{$\mathrm{C}_{3 h}$} & $\begin{array}{l}A^{\prime} \\
A^{\prime \prime}\end{array}$ & $\begin{array}{ll}1 & 1\end{array}$ & 1 & 1 & 1 & 1 & $\begin{array}{l}1 \\
2\end{array}$ & $\begin{array}{l}2 \\
1\end{array}$ & $\begin{array}{l}1 \\
2\end{array}$ & $\begin{array}{l}2 \\
1\end{array}$ & $\begin{array}{l}1 \\
2\end{array}$ & $\begin{array}{l}2 \\
1\end{array}$ & $\begin{array}{l}3 \\
2\end{array}$ & $\begin{array}{l}2 \\
3\end{array}$ & $\begin{array}{l}3 \\
2\end{array}$ & $\begin{array}{l}2 \\
3\end{array}$ & $\begin{array}{l}3 \\
2\end{array}$ & $\begin{array}{l}2 \\
3\end{array}$ \\
\hline & $\begin{array}{l}C_{1}^{\prime} \\
C_{1}^{\prime \prime}\end{array}$ & & 1 & 1 & $\begin{array}{l}1 \\
1\end{array}$ & $\begin{array}{l}1 \\
1\end{array}$ & $\begin{array}{l}1 \\
1\end{array}$ & $\begin{array}{l}1 \\
1\end{array}$ & $\begin{array}{l}2 \\
1\end{array}$ & $\begin{array}{l}1 \\
2\end{array}$ & $\begin{array}{l}2 \\
2\end{array}$ & $\begin{array}{l}2 \\
2\end{array}$ & $\begin{array}{l}2 \\
2\end{array}$ & $\begin{array}{l}2 \\
2\end{array}$ & $\begin{array}{l}2 \\
3\end{array}$ & $\begin{array}{l}3 \\
2\end{array}$ & $\begin{array}{l}3 \\
3\end{array}$ & $\begin{array}{l}3 \\
3\end{array}$ \\
\hline & $\begin{array}{l}C_{2}^{\prime} \\
C_{2}^{\prime \prime}\end{array}$ & & 1 & 1 & $\begin{array}{l}1 \\
1\end{array}$ & $\begin{array}{l}1 \\
1\end{array}$ & $\begin{array}{l}1 \\
1\end{array}$ & $\begin{array}{l}1 \\
1\end{array}$ & $\begin{array}{l}2 \\
1\end{array}$ & $\begin{array}{l}1 \\
2\end{array}$ & $\begin{array}{l}2 \\
2\end{array}$ & $\begin{array}{l}2 \\
2\end{array}$ & $\begin{array}{l}2 \\
2\end{array}$ & $\begin{array}{l}2 \\
2\end{array}$ & $\begin{array}{l}2 \\
3\end{array}$ & $\begin{array}{l}3 \\
2\end{array}$ & $\begin{array}{l}3 \\
3\end{array}$ & 3 \\
\hline
\end{tabular}

Note: The states $\left(C_{1}^{\prime}, C_{2}^{\prime}\right)$ and $\left(C_{1}^{\prime \prime}, C_{2}^{\prime \prime}\right)$ are Kramers conjugate states. 
TABLE 9. Type and number of perturbed levels-Continued

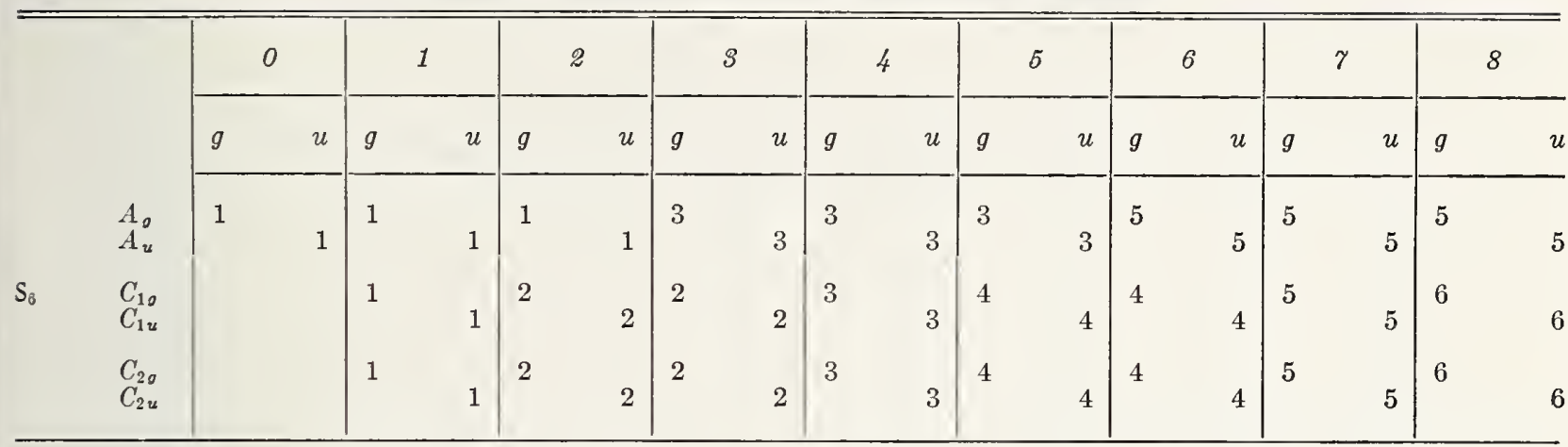

Note: The states $\left(C_{1 \sigma}, C_{2 \sigma}\right)$ and $\left(C_{1 u}, C_{2 u}\right)$ are Kramers conjugate states.

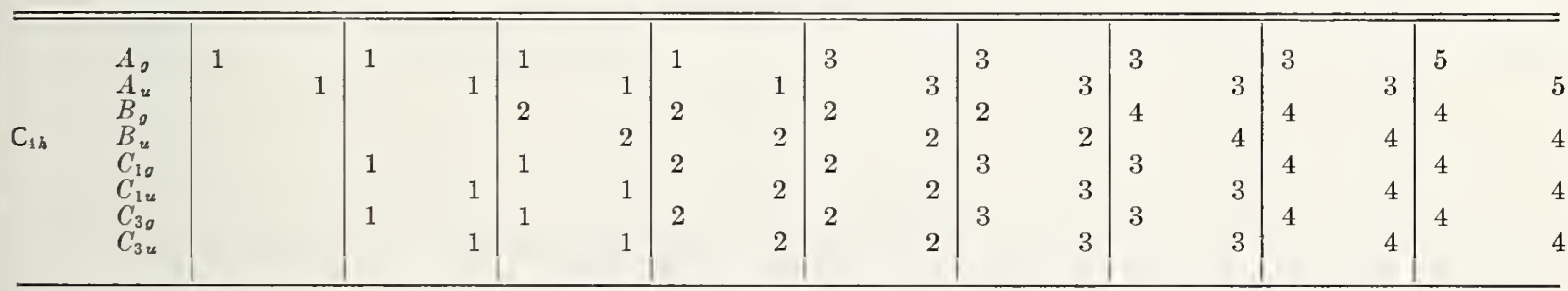

Note: The states $\left(C_{1 \vartheta}, C_{3 \vartheta}\right)$ and $\left(C_{1 u}, C_{3 u}\right)$ are Kramers conjugate states.

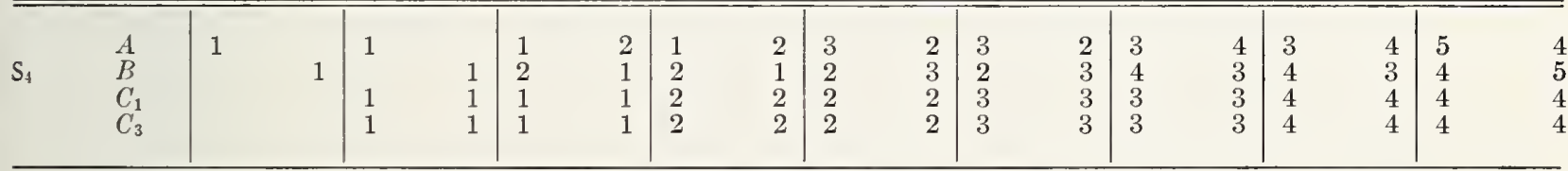

Note: The states $C_{1}$ and $C_{3}$ are Kramers conjugate states.

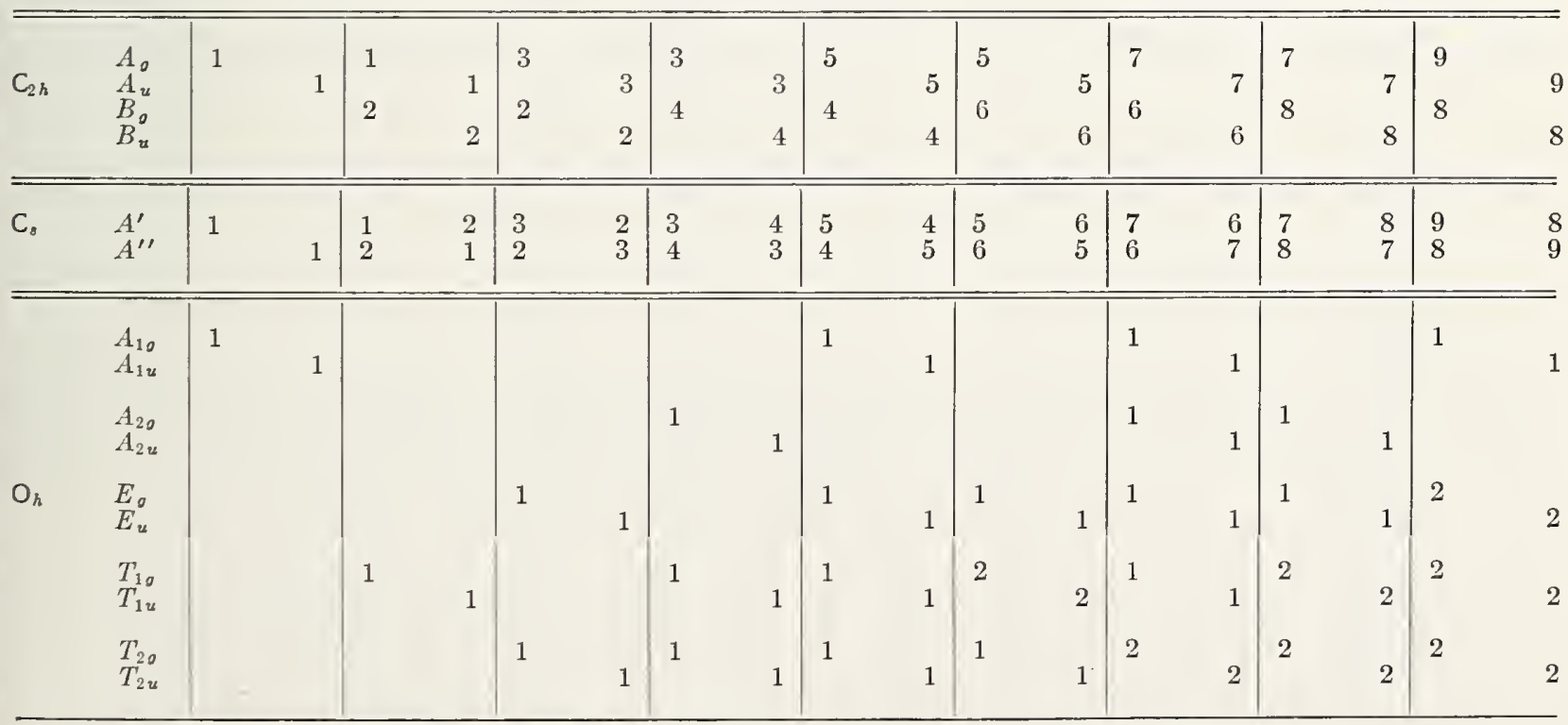


TABLE 9. Type and number of perturbed levels-Continued

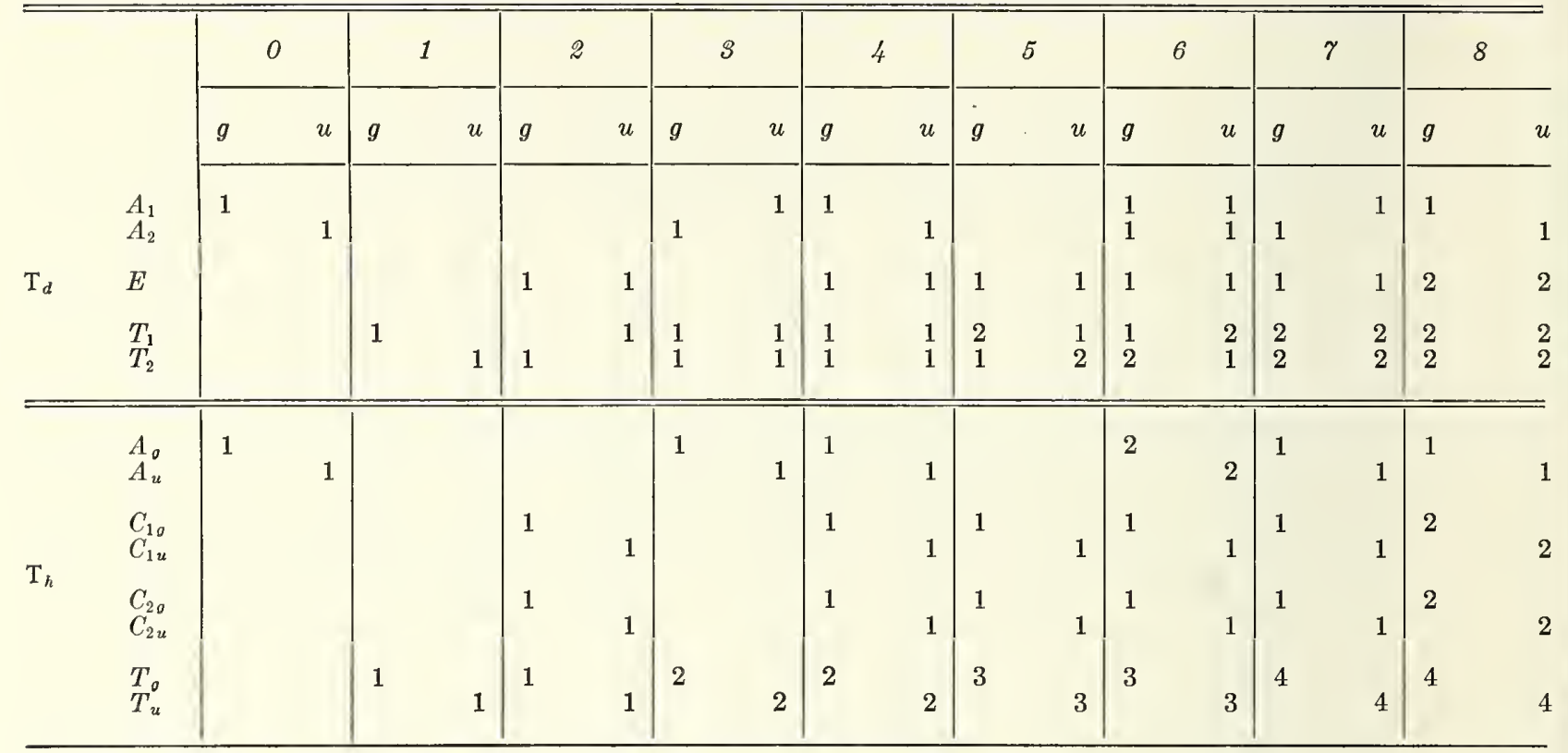

Note: The states $\left(C_{1 \vartheta}, C_{2 \vartheta}\right)$ and $\left(C_{1 u}, C_{2 u}\right)$ are Kramers conjugate states.

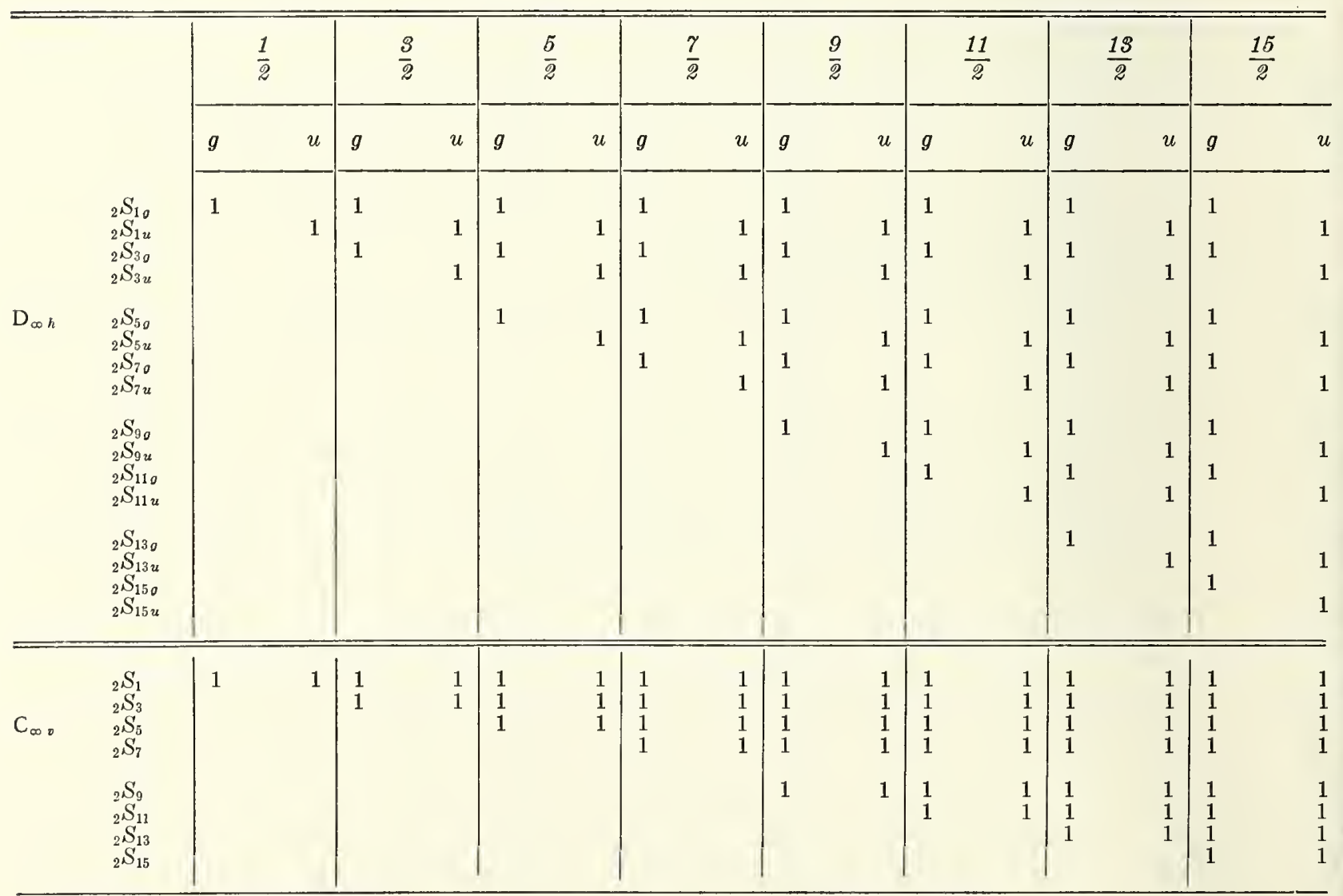


TABLE 9. Type and number of perturbed levels-Continued

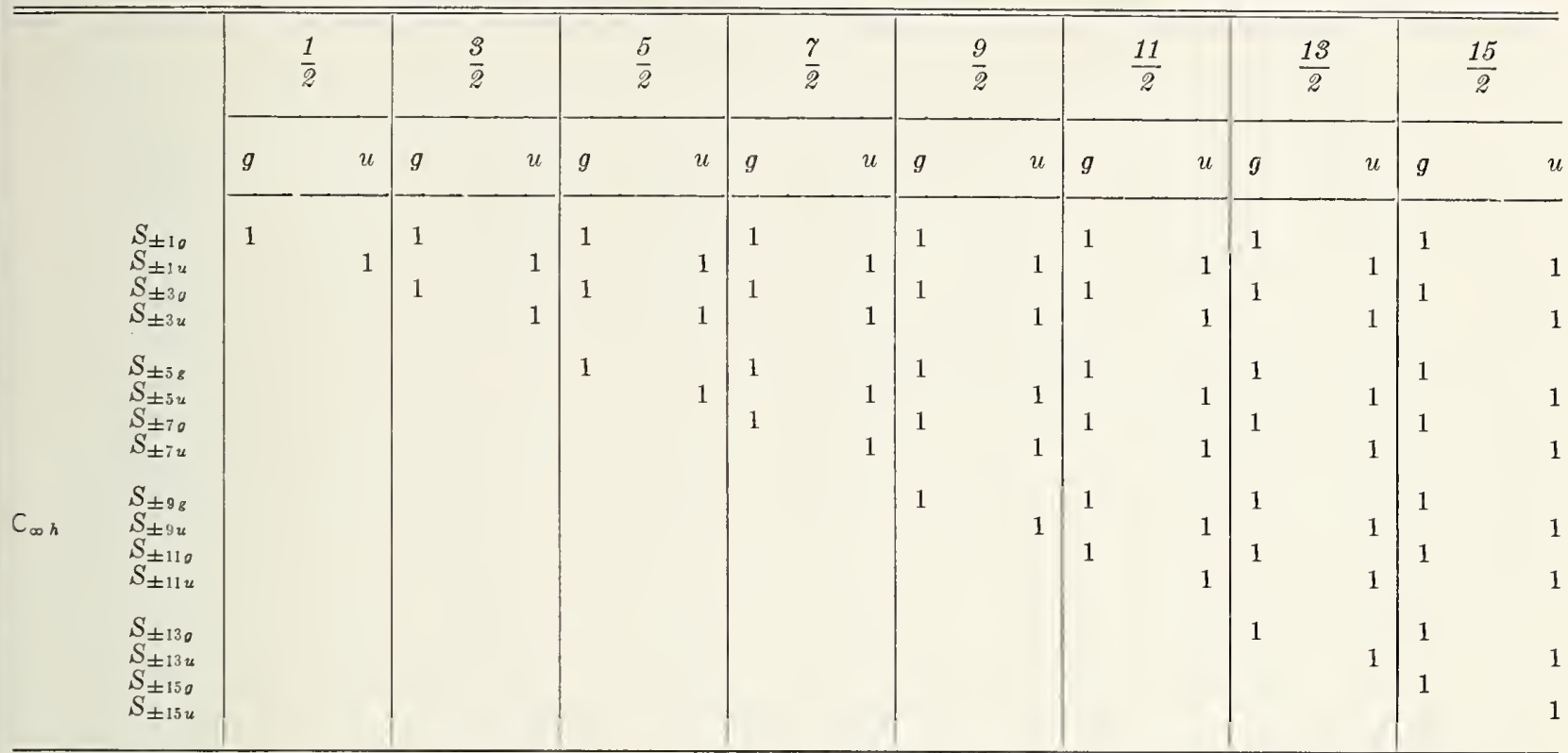

Note: The states $\left(S_{n_{0}}, S_{-n_{0}}\right)$ and $\left(S_{n u}, S_{-n u}\right)$ are Kramers conjugate states.

\begin{tabular}{|c|c|c|c|c|c|c|c|c|c|c|c|c|c|c|c|c|c|}
\hline $\mathrm{D}_{6 h}$ & $\begin{array}{l}\mathrm{D}_{1 / 19} \\
D_{1 / 2 u} \\
{ }_{2} S_{1 u} \\
{ }_{2} S_{1 u} \\
{ }_{2} S_{u g} \\
{ }_{2} S_{2 u}\end{array}$ & 1 & 1 & 1 & 1 & $\begin{array}{l}1 \\
1\end{array}$ & $\begin{array}{l}1 \\
1 \\
1\end{array}$ & $\begin{array}{l}1 \\
1 \\
2\end{array}$ & $\begin{array}{l}1 \\
1 \\
2\end{array}$ & $\begin{array}{l}1 \\
2 \\
2\end{array}$ & $\begin{array}{l}1 \\
2 \\
2\end{array}$ & $\begin{array}{l}2 \\
2 \\
2\end{array}$ & $\begin{array}{l}2 \\
2 \\
2\end{array}$ & $\begin{array}{l}3 \\
2 \\
2\end{array}$ & $\begin{array}{l}3 \\
2 \\
2\end{array}$ & $\begin{array}{l}3 \\
3 \\
2\end{array}$ & 2 \\
\hline $\mathrm{C}_{6 z}$ & $\begin{array}{l}D_{3 / 3} \\
{ }_{2} S_{i} \\
{ }_{2} S_{2}\end{array}$ & 1 & 1 & $\begin{array}{l}1 \\
1\end{array}$ & $\begin{array}{l}1 \\
1\end{array}$ & $\begin{array}{l}1 \\
1 \\
1\end{array}$ & $\begin{array}{l}1 \\
1 \\
1\end{array}$ & $\begin{array}{l}1 \\
1 \\
2\end{array}$ & $\begin{array}{l}1 \\
1 \\
2\end{array}$ & $\begin{array}{l}1 \\
2 \\
2\end{array}$ & $\begin{array}{l}1 \\
2 \\
2\end{array}$ & $\begin{array}{l}2 \\
2 \\
2\end{array}$ & $\begin{array}{l}2 \\
2 \\
2\end{array}$ & $\begin{array}{l}3 \\
2 \\
2\end{array}$ & $\begin{array}{l}3 \\
2 \\
2\end{array}$ & $\begin{array}{l}3 \\
3 \\
2\end{array}$ & $\begin{array}{l}3 \\
3 \\
2\end{array}$ \\
\hline $\mathrm{D}_{3 h}$ & $\begin{array}{l}D_{1 / 2} \\
{ }_{2} S_{1} \\
{ }_{2} S_{2}\end{array}$ & 1 & 1 & $\begin{array}{l}1 \\
1\end{array}$ & $\begin{array}{l}1 \\
1\end{array}$ & $\begin{array}{l}1 \\
1 \\
1\end{array}$ & $\begin{array}{l}1 \\
1 \\
1\end{array}$ & $\begin{array}{l}1 \\
1 \\
2\end{array}$ & $\begin{array}{l}2 \\
1 \\
1\end{array}$ & $\begin{array}{l}1 \\
2 \\
2\end{array}$ & $\begin{array}{l}2 \\
2 \\
1\end{array}$ & $\begin{array}{l}2 \\
2 \\
2\end{array}$ & $\begin{array}{l}2 \\
2 \\
2\end{array}$ & $\begin{array}{l}3 \\
2 \\
2\end{array}$ & $\begin{array}{l}2 \\
2 \\
3\end{array}$ & $\begin{array}{l}3 \\
3 \\
2\end{array}$ & $\begin{array}{l}2 \\
3 \\
3\end{array}$ \\
\hline$D_{i k}$ & $\begin{array}{l}D_{3 / 0} \\
D_{3 / 2 u} \\
{ }_{2} S_{0} \\
{ }_{2} S_{u}\end{array}$ & 1 & 1 & $\begin{array}{l}1 \\
1\end{array}$ & $\begin{array}{l}1 \\
1\end{array}$ & $\begin{array}{l}1 \\
2\end{array}$ & $\begin{array}{l}1 \\
2\end{array}$ & $\begin{array}{l}2 \\
2\end{array}$ & $\begin{array}{l}2 \\
2\end{array}$ & $\begin{array}{l}3 \\
2\end{array}$ & $\begin{array}{l}3 \\
2\end{array}$ & $\begin{array}{l}3 \\
3\end{array}$ & $\begin{array}{l}3 \\
3\end{array}$ & $\begin{array}{l}3 \\
4\end{array}$ & $\begin{array}{l}3 \\
4\end{array}$ & $\begin{array}{l}4 \\
4\end{array}$ & $\begin{array}{l}4 \\
4\end{array}$ \\
\hline$C_{40}$ & ${ }_{2 S}^{D_{3 / 2}}$ & 1 & 1 & $\begin{array}{l}1 \\
1\end{array}$ & $\begin{array}{l}1 \\
1\end{array}$ & $\begin{array}{l}1 \\
2\end{array}$ & $\begin{array}{l}1 \\
2\end{array}$ & $\begin{array}{l}2 \\
2\end{array}$ & $\begin{array}{l}2 \\
2\end{array}$ & $\begin{array}{l}3 \\
2\end{array}$ & $\begin{array}{l}3 \\
2\end{array}$ & $\begin{array}{l}3 \\
3\end{array}$ & $\begin{array}{l}3 \\
3\end{array}$ & $\begin{array}{l}3 \\
4\end{array}$ & $\begin{array}{l}3 \\
4\end{array}$ & $\begin{array}{l}4 \\
4\end{array}$ & $\begin{array}{l}4 \\
4\end{array}$ \\
\hline $\mathrm{D}_{2 d}$ & $\begin{array}{l}D_{1 / 2} \\
{ }_{2} S\end{array}$ & 1 & 1 & 1 & $\begin{array}{l}1 \\
1\end{array}$ & $\begin{array}{l}1 \\
2\end{array}$ & $\begin{array}{l}2 \\
1\end{array}$ & $\begin{array}{l}2 \\
2\end{array}$ & $\begin{array}{l}2 \\
2\end{array}$ & $\begin{array}{l}3 \\
2\end{array}$ & $\begin{array}{l}2 \\
3\end{array}$ & $\begin{array}{l}3 \\
3\end{array}$ & $\begin{array}{l}3 \\
3\end{array}$ & $\begin{array}{l}3 \\
4\end{array}$ & $\begin{array}{l}4 \\
3\end{array}$ & $\begin{array}{l}4 \\
4\end{array}$ & $\begin{array}{l}4 \\
4\end{array}$ \\
\hline $\mathrm{D}_{3 d}$ & $\begin{array}{l}D_{1 / 2 u} \\
D_{1 / 2 u} \\
S_{10} \\
S_{1 u} \\
S_{3 o} \\
S_{3 u}\end{array}$ & 1 & 1 & 1 & $\begin{array}{l}1 \\
1 \\
1\end{array}$ & $\begin{array}{l}2 \\
1 \\
1\end{array}$ & $\begin{array}{l}2 \\
1 \\
1\end{array}$ & $\begin{array}{l}3 \\
1 \\
1\end{array}$ & $\begin{array}{l}3 \\
1 \\
1\end{array}$ & $\begin{array}{l}3 \\
2 \\
2\end{array}$ & $\begin{array}{l}3 \\
2 \\
2\end{array}$ & $\begin{array}{l}4 \\
2 \\
2\end{array}$ & $\begin{array}{l}4 \\
2 \\
2\end{array}$ & $\begin{array}{l}5 \\
2 \\
2\end{array}$ & $\begin{array}{l}5 \\
2 \\
2\end{array}$ & $\begin{array}{l}5 \\
3 \\
3\end{array}$ & $\begin{array}{l}5 \\
3 \\
3\end{array}$ \\
\hline
\end{tabular}

Note: The states $\left(S_{1 \vartheta}, S_{30}\right)$ and $\left(S_{1 u}, S_{3 u}\right)$ are Kramers conjugate states. 
TABLE 9. Type and number of perturbed levels-Continued

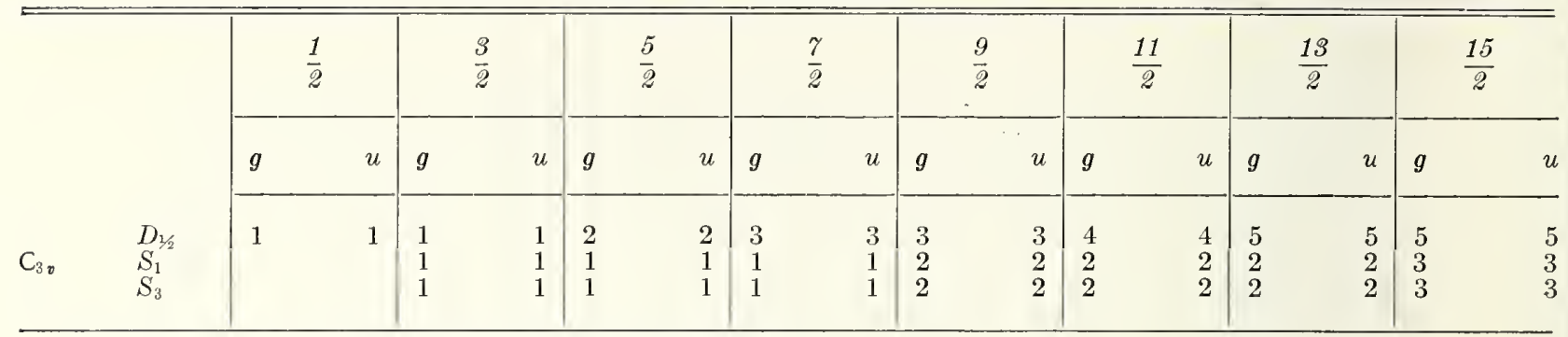

Note: The states $\left(S_{1}, S_{3}\right)$ are Kramers conjugate states.

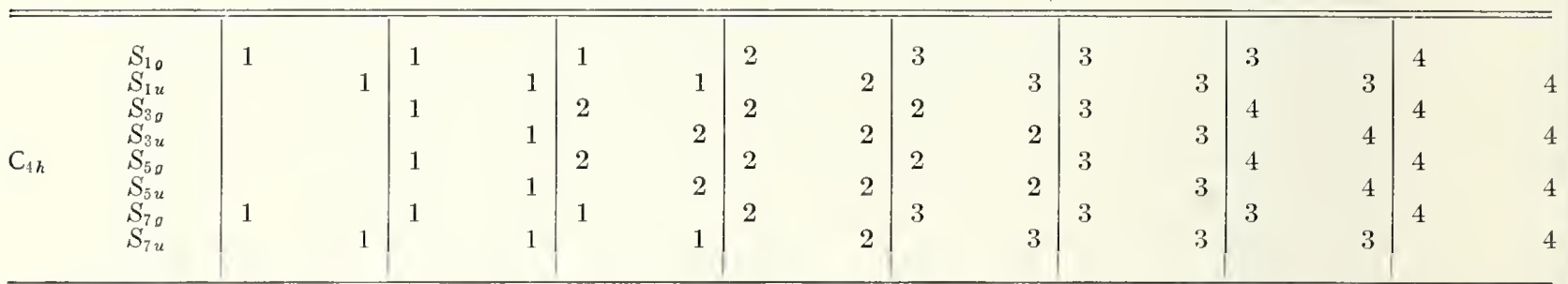

Note: The states $\left(S_{1 g}, S_{7 g}\right),\left(S_{1 u}, S_{7 u}\right),\left(S_{3 g}, S_{5 q}\right),\left(S_{3 u}, S_{5 u}\right)$ are Kramers conjugate states.

\begin{tabular}{|c|c|c|c|c|c|c|c|c|c|c|c|c|c|c|c|c|c|}
\hline$S_{t}$ & $\begin{array}{l}S_{1} \\
S_{3} \\
S_{5} \\
S_{7}\end{array}$ & $\begin{array}{l}1 \\
1\end{array}$ & 1 & $\begin{array}{l}1 \\
1 \\
1 \\
1\end{array}$ & $\begin{array}{l}1 \\
1 \\
1 \\
1\end{array}$ & $\begin{array}{l}2 \\
1 \\
1 \\
2\end{array}$ & $\begin{array}{l}1 \\
2 \\
2 \\
1\end{array}$ & $\begin{array}{l}2 \\
2 \\
2 \\
2\end{array}$ & $\begin{array}{l}2 \\
2 \\
2 \\
2\end{array}$ & $\begin{array}{l}2 \\
3 \\
3 \\
2\end{array}$ & $\begin{array}{l}3 \\
2 \\
2 \\
3\end{array}$ & $\begin{array}{l}3 \\
3 \\
3 \\
3\end{array}$ & $\begin{array}{l}3 \\
3 \\
3 \\
3\end{array}$ & $\begin{array}{l}4 \\
3 \\
3 \\
4\end{array}$ & $\begin{array}{l}3 \\
4 \\
4 \\
3\end{array}$ & $\begin{array}{l}4 \\
4 \\
4 \\
4\end{array}$ & $\begin{array}{l}4 \\
4 \\
4\end{array}$ \\
\hline
\end{tabular}

Note: The states $\left(S_{1}, S_{7}\right)$ and $\left(S_{2}, S_{5}\right)$ are Kramers conjugate states.

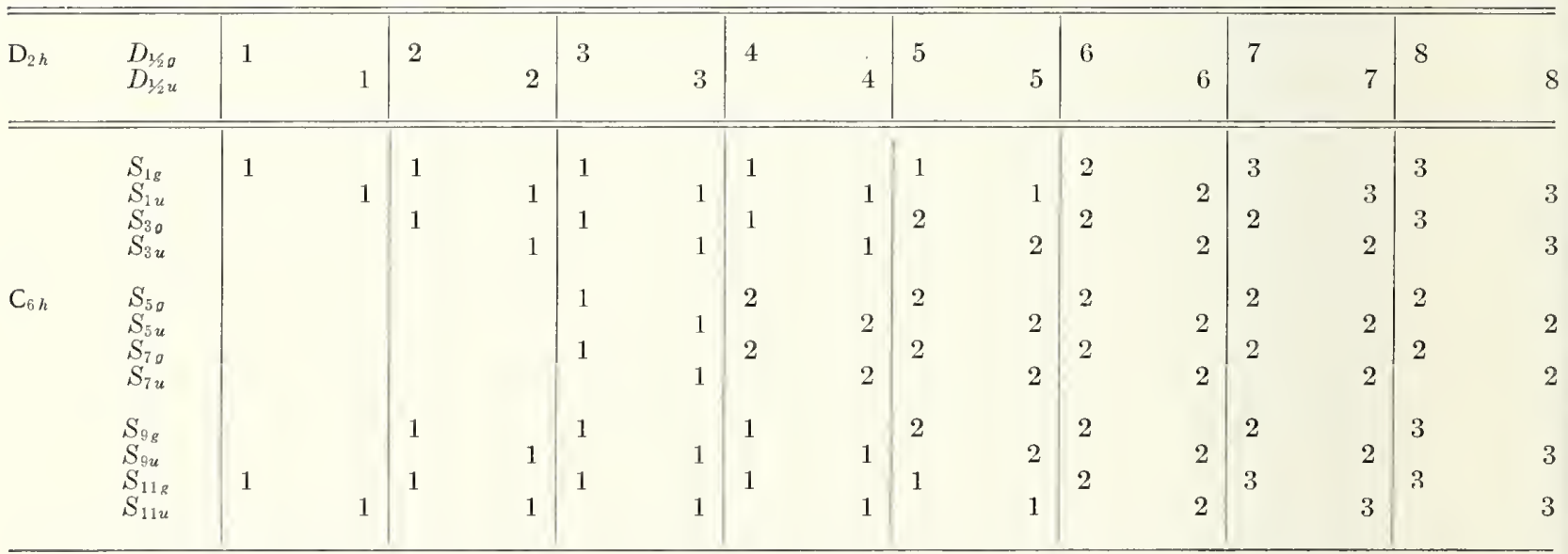

Note: The states $\left(S_{1 g}, S_{11 g}\right),\left(S_{1 u}, S_{11 u}\right),\left(S_{3 q}, S_{9 g}\right),\left(S_{3 u}, S_{9 u}\right),\left(S_{5 g}, S_{7 g}\right),\left(S_{5 u}, S_{7 u}\right)$ are Kramers conjugate states.

\begin{tabular}{|c|c|c|c|c|c|c|c|c|c|c|c|c|c|c|c|c|c|}
\hline \multirow{3}{*}{$C_{3 h}$} & $\begin{array}{l}S_{1} \\
S_{3}\end{array}$ & 1 & & $\begin{array}{l}1 \\
1\end{array}$ & 1 & $\begin{array}{l}1 \\
1\end{array}$ & $\begin{array}{l}1 \\
1\end{array}$ & $\begin{array}{l}1 \\
1\end{array}$ & $\begin{array}{l}2 \\
1\end{array}$ & $\begin{array}{l}1 \\
2\end{array}$ & $\begin{array}{l}2 \\
2\end{array}$ & $\begin{array}{l}2 \\
2\end{array}$ & $\begin{array}{l}2 \\
2\end{array}$ & $\begin{array}{l}3 \\
2\end{array}$ & $\begin{array}{l}2 \\
2\end{array}$ & $\begin{array}{l}3 \\
3\end{array}$ & $\begin{array}{l}2 \\
3\end{array}$ \\
\hline & $\begin{array}{l}S_{5} \\
S_{7}\end{array}$ & & $\begin{array}{l}1 \\
1\end{array}$ & & $\begin{array}{l}1 \\
1\end{array}$ & $\begin{array}{l}1 \\
1\end{array}$ & $\begin{array}{l}1 \\
1\end{array}$ & $\begin{array}{l}2 \\
2\end{array}$ & $\begin{array}{l}1 \\
1\end{array}$ & $\begin{array}{l}2 \\
2\end{array}$ & $\begin{array}{l}1 \\
1\end{array}$ & $\begin{array}{l}2 \\
2\end{array}$ & $\begin{array}{l}2 \\
2\end{array}$ & $\begin{array}{l}2 \\
2\end{array}$ & $\begin{array}{l}3 \\
3\end{array}$ & $\begin{array}{l}2 \\
2\end{array}$ & $\begin{array}{l}3 \\
3\end{array}$ \\
\hline & $\begin{array}{l}S_{9} \\
S_{11}\end{array}$ & 1 & & $\begin{array}{l}1 \\
1\end{array}$ & 1 & $\begin{array}{l}1 \\
1\end{array}$ & $\begin{array}{l}1 \\
1\end{array}$ & $\begin{array}{l}1 \\
1\end{array}$ & $\begin{array}{l}1 \\
2\end{array}$ & $\begin{array}{l}2 \\
1\end{array}$ & $\begin{array}{l}2 \\
2\end{array}$ & $\begin{array}{l}2 \\
2\end{array}$ & $\begin{array}{l}2 \\
2\end{array}$ & $\begin{array}{l}2 \\
3\end{array}$ & $\begin{array}{l}2 \\
2\end{array}$ & $\begin{array}{l}3 \\
3\end{array}$ & $\begin{array}{l}3 \\
2\end{array}$ \\
\hline
\end{tabular}

Note: The states $\left(S_{1}, S_{11}\right),\left(S_{3}, S_{9}\right),\left(S_{5}, S_{7}\right)$ are Kramers conjugate states. 
TABLE 9. Type and number of perturbed levels-Continued

\begin{tabular}{|c|c|c|c|c|c|c|c|c|c|c|c|c|c|c|c|c|c|}
\hline & & & & & & & & & & & & & & & & & \\
\hline & & $g$ & $u$ & $g$ & $u$ & $g$ & $u$ & $g$ & $u$ & $g$ & $u$ & $g$ & $u$ & $g$ & $u$ & $g$ & $u$ \\
\hline & $\begin{array}{l}S_{1 g} \\
S_{1 u}\end{array}$ & 1 & 1 & 1 & 1 & 2 & 2 & 3 & 3 & 3 & 3 & 4 & 4 & 5 & 5 & 5 & 5 \\
\hline $\mathrm{S}_{6}$ & $\begin{array}{l}S_{3 \sigma} \\
S_{3 u}\end{array}$ & & & 2 & 2 & 2 & 2 & 2 & 2 & 4 & 4 & 4 & 4 & 4 & 4 & 6 & 6 \\
\hline & $\begin{array}{l}S_{50} \\
S_{5 u}\end{array}$ & 1 & 1 & 1 & 1 & 2 & 2 & 3 & 3 & 3 & 3 & 4 & 4 & 5 & 5 & 5 & 5 \\
\hline
\end{tabular}

Note: The states $\left(S_{10}, S_{5 \sigma}\right),\left(S_{1 u}, S_{5 u}\right)$ are Kramers conjugate states. The states $\left(S_{3 o}, S_{3 u}\right)$ appear an even number of times as Kramers conjugate pairs.

\begin{tabular}{|c|c|c|c|c|c|c|c|c|c|c|c|c|c|c|c|c|c|}
\hline$C_{2}$, & $D_{3 / 2}$ & 1 & 1 & 2 & 2 & 3 & 3 & 4 & 4 & 5 & 5 & 6 & 6 & 7 & 7 & 8 & 8 \\
\hline $\mathrm{C}_{2 \hbar}$ & $\begin{array}{l}S_{10} \\
S_{1 u} \\
S_{30} \\
S_{3 u}\end{array}$ & $\begin{array}{l}1 \\
1\end{array}$ & $\begin{array}{l}1 \\
1\end{array}$ & $\begin{array}{l}2 \\
2\end{array}$ & $\begin{array}{l}2 \\
2\end{array}$ & $\begin{array}{l}3 \\
3\end{array}$ & $\begin{array}{l}3 \\
3\end{array}$ & $\begin{array}{l}4 \\
4\end{array}$ & $\begin{array}{l}4 \\
4\end{array}$ & $\begin{array}{l}5 \\
5\end{array}$ & $\begin{array}{l}5 \\
5\end{array}$ & $\begin{array}{l}6 \\
6\end{array}$ & $\begin{array}{l}6 \\
6\end{array}$ & $\begin{array}{l}7 \\
7\end{array}$ & $\begin{array}{l}7 \\
7\end{array}$ & $\begin{array}{l}8 \\
8\end{array}$ & $\begin{array}{l}8 \\
8\end{array}$ \\
\hline
\end{tabular}

Note: The states $\left(S_{1 \sigma}, S_{3 ø}\right)$ and $\left(S_{1 u}, S_{3 u}\right)$ are Kramers conjugate states.

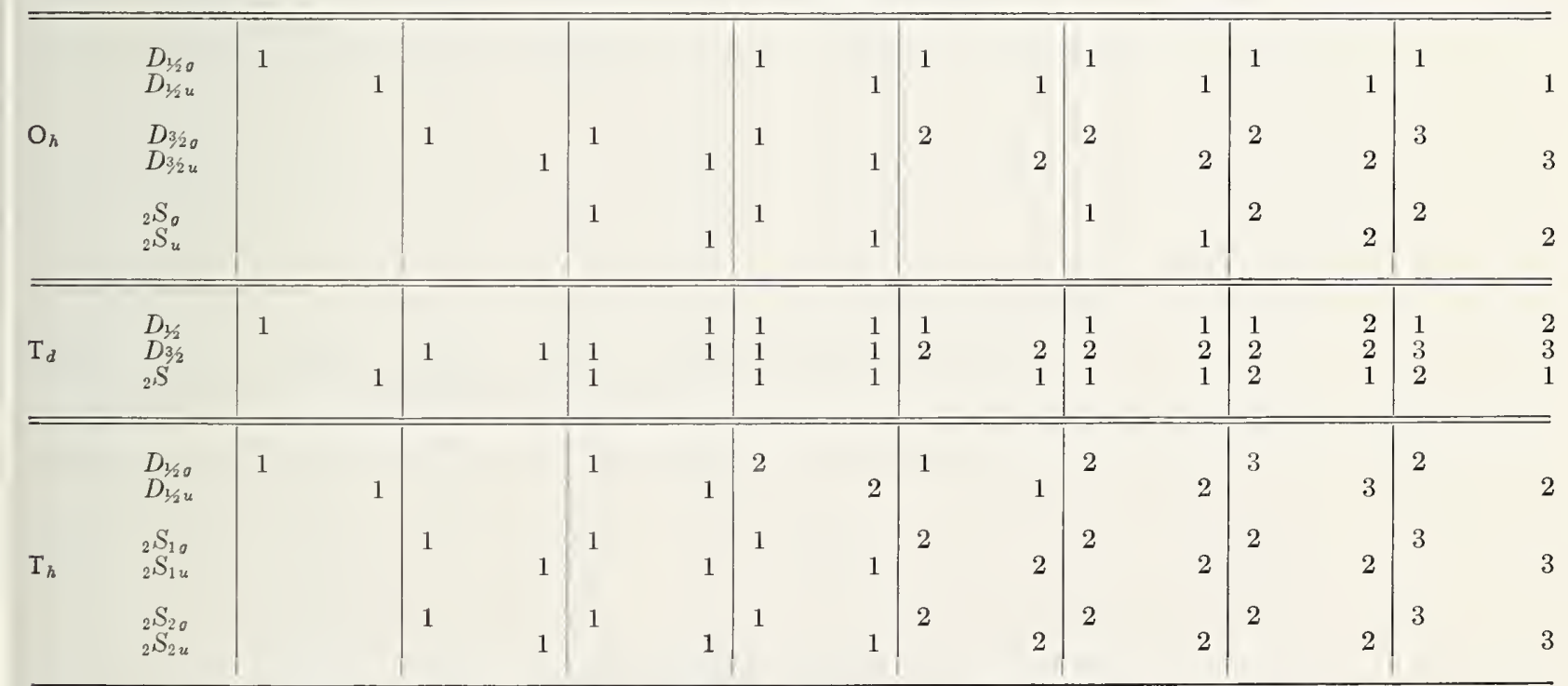

Note: The states $\left({ }_{2} S_{1 g},{ }_{2} S_{2 g}\right)$ and $\left({ }_{2} S_{1 u},{ }_{2} S_{2 u}\right)$ are Kramers conjugate pairs of states. 


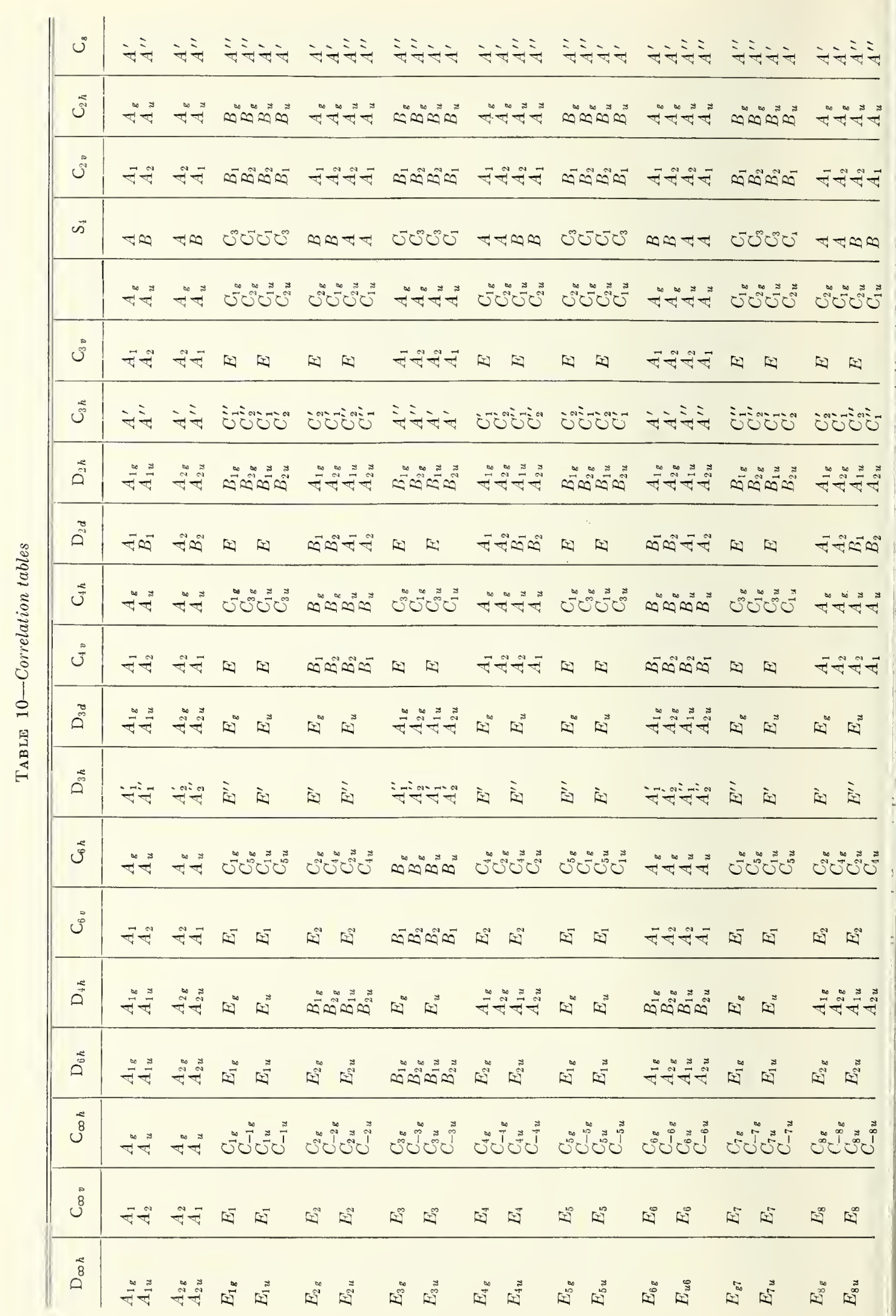


$\mathrm{D}_{2 h}$ where table 5 shows that potential coefficients $C_{2}^{0} C_{4}^{0} C_{6}^{0} ; C_{2}^{2} C_{4}^{2} C_{6}^{2} ; C_{4}^{4} C_{6}^{4}$ and $C_{6}^{6}$ are present, and table 9 shows that there are two states $A_{1 u}$ and one each of $A_{2 u}, B_{1 u}$, and $B_{2 u}$, indicating that the original fivefold degeneracy of the state $2_{u}$ has been destroyed. If the terms $C_{2}^{0}, C_{4}^{0}$, and $C_{6}^{0}$ are more significant than the rest, an initial approximation using these terms only leads to $\mathrm{D}_{\infty h}$ symmetry, with states $A_{1 u}, E_{1 u}$, and $E_{2 u}$. The inclusion of $C_{6}^{6}$ will reduce the symmetry to $D_{6 h}$, but the states retain their designation. On the other hand, the inclusion of $C_{4}^{4}$ will reduce the symmetry to $\mathrm{D}_{4 h}$, leave the $A_{1 u}$ level unaffected, leave the $E_{1 u}$ level unaffected save for a redesignation to $E_{u}$, and cause the $E_{2 u}$ level to split to $B_{1 u}$ and $B_{2 u}$. If we now include all terms, we see that $E_{u}$ of $\mathrm{D}_{4 h}$ splits to $B_{1 u}$ and $B_{2 u}$ of $\mathrm{D}_{2 h}$, and that the states $B_{1 u}$ and $B_{2 u}$ of $\mathrm{D}_{4 h}$ become $A_{1 u}$ and $A_{2 u}$, respectively, in $\mathrm{D}_{2 h}$. This $A_{1 u}$ state can interact with the $A_{1 u}$ state from the original $\mathrm{D}_{\infty} h$ approximation, so that a rigid correlation with the states of $\mathrm{D}_{\infty h}$ has been lost.

Table 10 has been so constructed that the same process may be followed for still lower order groups. For every column in the table, its subgroups are found further to the right in the table (or in the same column without the " $g$ " - " $u$ "), and the designation of any level under increasingly lower symmetry may be followed across the table. Entries of the same line have significance only with respect to the allowed subgroups of any given group. Thus, there is no correlation between parallel entries in $D_{6 h}$ and $D_{4 h}$, though either may be correlated with $\mathrm{D}_{2 h}$. All splittings of doubledegenerate reps have been correlated to ensure consistency. Since the basic entries of the table are the reps of $\mathrm{D}_{\infty}$, any desired rep of some other group must be found from the table by inspection but its behavior under lower symmetry does not depend upon the rep of $\mathrm{D}_{\infty h}$ from which it arose. In a manner of speaking, the columns of table 10 are first differences of the entries of table 9 .

Table 11 shows the reduction of the reps of the cubic group into the reps of their own cubic subgroups, and into reps of the noncubic groups. Here, we have considered the two possibilities which may arise in destroying cubic symmetry. Thus, the group $\mathrm{O}$ may be reduced to $\mathrm{D}_{4}$ by destroying the threefold axes, or to $\mathrm{D}_{3}$ by destroying the fourfold axes. In either case, the principal axis remaining is considered to be the principal axis of symmetry.

While our discussion has been that appropriate to the weak-field case (sections 3.3, 3.4), the results are immediately applicable to both the intermediate field case and the strong field case. In the former, the external perturbation is stronger than the spin-orbit interaction. Consequently, instead of reducing the representation $D_{L} \times D_{S}$ into those reps of the group leaving invariant the spin-orbit term in the Hamiltonian, i.e., $D_{J}$, we reduce the representation into those reps of the group leaving invariant the crystal field potential $V$. Since the spin is not included in the Hamiltonian at this stage of the approximation, we reduce $D_{L}$ only into reps $X_{i}$. This may be done by using table 9 for $L$ instead of $J$ (here, the double groups will not appear, since $L$ must be integral). The rep for the wave function, including spin, will then be $X_{i} \times D_{S}$. We shall parallel spectroscopic notation for such a state by using the appropriate symbol from table 8 for the rep $X_{i}$, with an anterior superscript $2 S+1$ for $D_{S}$, the whole being placed in parentheses. If the spin-orbit interaction is now intro-

TABLE 11. Reduction of the cubic groups

\begin{tabular}{|c|c|c|c|c|c|c|c|c|c|c|c|}
\hline$\widehat{\mathrm{O}_{h}}$ & $\mathrm{~T}_{h}$ & $\mathrm{~T}_{d}$ & $\mathrm{D}_{4 h}$ & $\overrightarrow{\mathrm{D}}_{3 d}$ & $\overline{\mathrm{T}_{h}}$ & $\mathrm{D}_{2 h}$ & $\vec{S}_{6}$ & $\overline{\mathrm{T}_{d}}$ & $\mathrm{~T}$ & $\mathrm{D}_{2 d}$ & $\overrightarrow{\mathrm{C}}_{3 \text { च }}$ \\
\hline $\begin{array}{l}A_{18} \\
A_{1 u}\end{array}$ & $\begin{array}{l}A_{g} \\
A_{u}\end{array}$ & $\begin{array}{l}A_{1} \\
A_{2}\end{array}$ & $\begin{array}{l}A_{1 g} \\
A_{1 u}\end{array}$ & $\begin{array}{l}A_{18} \\
A_{1 u}\end{array}$ & $\begin{array}{l}A_{g} \\
A_{u}\end{array}$ & $\begin{array}{l}A_{1 g} \\
A_{1 u}\end{array}$ & $\begin{array}{l}A_{g} \\
A_{u}\end{array}$ & $\begin{array}{l}A_{1} \\
A_{2}\end{array}$ & $\begin{array}{l}A \\
A\end{array}$ & $\begin{array}{l}A_{1} \\
B_{1}\end{array}$ & $\begin{array}{l}A_{1} \\
A_{2}\end{array}$ \\
\hline $\begin{array}{l}A_{2 z} \\
A_{2 u}\end{array}$ & $\begin{array}{l}A_{g} \\
A_{u}\end{array}$ & $\begin{array}{l}A_{2} \\
A_{1}\end{array}$ & $\begin{array}{l}B_{1 g} \\
B_{1 u}\end{array}$ & $\begin{array}{l}A_{2 g} \\
A_{2 u}\end{array}$ & $\begin{array}{l}C_{1 g} \\
C_{1 u}\end{array}$ & $\begin{array}{l}A_{1 g} \\
A_{1 u}\end{array}$ & $\begin{array}{l}C_{1 g} \\
C_{1 u}\end{array}$ & $\begin{array}{l}E \\
T_{1}\end{array}$ & $\begin{array}{l}C_{1} C_{2} \\
T\end{array}$ & $\begin{array}{l}A_{1} B_{1-} \\
A_{2} E\end{array}$ & $\begin{array}{l}E \\
A_{2} E\end{array}$ \\
\hline $\begin{array}{l}E_{g} \\
E_{u}\end{array}$ & $\begin{array}{l}C_{1 g} C_{2 g} \\
C_{1 u} C_{2 u}\end{array}$ & $\begin{array}{l}E \\
E\end{array}$ & $\begin{array}{l}A_{1 g} B_{1 g} \\
A_{1 u} B_{1 u}\end{array}$ & $\begin{array}{l}E_{g} \\
E_{u}\end{array}$ & $\begin{array}{l}C_{2 g} \\
C_{2 u}\end{array}$ & $\begin{array}{l}A_{1 g} \\
A_{1 u}\end{array}$ & $\begin{array}{l}C_{2 g} \\
C_{2 u}\end{array}$ & $T_{2}$ & & & $A_{1} E$ \\
\hline $\begin{array}{l}T_{1 g} \\
T_{1 u}\end{array}$ & $\begin{array}{l}T_{u} \mathrm{C}^{u} \\
T_{g} \\
T_{u}\end{array}$ & $\begin{array}{l}T_{1} \\
T_{2}\end{array}$ & $\begin{array}{l}E_{g} A_{2 g} \\
E_{u} A_{2 u}\end{array}$ & $\begin{array}{l}E_{g} A_{2 g} \\
E_{u} A_{2 u}\end{array}$ & $\begin{array}{l}C_{2 u} \\
T_{g} \\
T_{u}\end{array}$ & $\begin{array}{l}A_{1 u} \\
A_{2 g} B_{1 g} B_{2 g} \\
A_{2 u} B_{1 u} B_{2 u}\end{array}$ & $\begin{array}{l}A_{g} C_{1 g} C_{2 g} \\
A_{u} C_{1 u} C_{2 u}\end{array}$ & $\begin{array}{l}D_{1 / 2} \\
2 S^{2} \\
D_{3 / 2}\end{array}$ & $\begin{array}{l}D_{3 / 2} \\
D_{1 / 2} \\
{ }_{2} S_{12} S_{2}\end{array}$ & $\begin{array}{l}D_{3 / 2} \\
{ }_{2} S^{2} \\
D_{3 / 2} S\end{array}$ & $\begin{array}{l}D_{3 / 2} \\
D_{1 / 2} \\
D_{3 / 2} S_{1} S_{3}\end{array}$ \\
\hline $\begin{array}{l}T_{2 g} \\
T_{2 u}\end{array}$ & $\begin{array}{l}T_{g} \\
T_{u}\end{array}$ & $\begin{array}{l}T_{2} \\
T_{1}\end{array}$ & $\begin{array}{l}E_{g} B_{2 g} \\
E_{u} B_{2 u}\end{array}$ & $\begin{array}{l}E_{g} A_{1 g} \\
E_{u} A_{1 u}\end{array}$ & $\begin{array}{l}D_{1 / 28} \\
D_{3 / 2 u}\end{array}$ & $\begin{array}{l}D_{1 / 2 g} g \\
D_{1 / 2 u}^{1 / 2}\end{array}$ & $\begin{array}{l}S_{1 g} S_{5 g} \\
S_{1 u} S_{5 u}\end{array}$ & & & & \\
\hline $\begin{array}{l}D_{1 / 2 g} \\
D_{1 / 2 u}\end{array}$ & $\begin{array}{l}D_{1 / 28} \\
D_{1 / 2 u}\end{array}$ & $\begin{array}{l}D_{1 / 2} \\
{ }_{2} S^{2}\end{array}$ & $\begin{array}{l}D_{1 / 2 g} \\
D_{1 / 2 u}^{1 / 2}\end{array}$ & $\begin{array}{l}D_{1 / 2 g} \\
D_{3 / 2 u}\end{array}$ & $\begin{array}{l}{ }_{2} S_{1 g} \\
{ }_{2} S_{1 u}\end{array}$ & $\begin{array}{l}D_{1 / 28} \\
D_{1 / 2 u}\end{array}$ & $\begin{array}{l}S_{1 g} S_{3 g} \\
S_{1 u} S_{3 u}\end{array}$ & & & & \\
\hline${ }_{2} S_{g} S_{u}$ & $\begin{array}{l}D_{1 / 2 g} \\
D_{1 / 2 u}\end{array}$ & $\stackrel{2 S}{D_{1 / 2}}$ & ${ }_{2} S_{S_{g}}$ & $\begin{array}{l}D_{3 / 2 g} \\
D_{3 / 2 u}\end{array}$ & $\begin{array}{l}{ }_{2} S_{2 g} \\
{ }_{2} S_{2 u}\end{array}$ & $\begin{array}{l}D_{1 / 28} \\
D_{1 / 2 u}\end{array}$ & $\begin{array}{l}S_{3 g} S_{5 g} \\
S_{3 u} S_{5 u}\end{array}$ & & & & \\
\hline $\begin{array}{l}D_{3 / 2 g} \\
D_{3 / 2 u}\end{array}$ & $\begin{array}{ll}{ }_{2} S_{1 g} S_{2} S_{2 g} \\
{ }_{2} S_{1 u}{ }_{2} S_{2 u}\end{array}$ & $\begin{array}{l}D_{3 / 2} \\
D_{3 / 2}\end{array}$ & $\begin{array}{ll}D_{3 / 2 g} & { }_{2} S_{g} \\
D_{3 / 2 u} & S_{2} S_{u}\end{array} \mid$ & 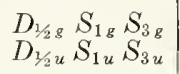 & & & & & & & \\
\hline
\end{tabular}


duced, the rep $X_{i} \times D_{S}$ is now merely a representation, so that it must be reduced according to the overall symmetry of the problem, which is still that of the crystal field $V$. If $X_{i}$ is one-dimensional, the splitting which arises is that of the spin states only through $D_{S}$, and the angular momentum is said to be quenched; if $X_{i}$ is of dimensionality greater than 1 but less than $2 L+1$, the orbital angular momentum is partially quenched. This verbally depicts the effect on the orbital angular momentum of the strong crystal potential field $V$, which destroys ( $X_{i}$ one-dimensional) or limits $\left(1<X_{i E}<2 L+1\right)$ any further influence on the angular momentum either by spin-orbit interaction or by a moderate external magnetic field. Still another intuitive view is that the crystal potential partially or completely fixes the orientation of the angular momentum in the lattice, so that these other influences have no further effect.

As an example, let us consider the ${ }^{7} F_{2}$ level of the europium trivalent ion, the symbol indicating a reduction of $D_{3} \times D_{3}$ to $D_{2}$ by spin-orbit interaction. A weak perturbing field $D_{4 h}$ will (table 9) split this $2_{g}$ level into states described as $\left({ }^{7} F^{7}\right) A_{1 g} ;\left({ }^{7} F^{7}\right) B_{1 g} ;\left({ }^{7} F^{7}\right) B_{2 g} ;$ and $\left({ }^{7} F^{7}\right) E_{g}$. An intermediate case field $\mathrm{D}_{4 h}$ will split the ${ }^{7} F^{\top}$ term (table 9 for $\left.B_{g}\right)$ into $\left({ }^{7} A_{2 g}\right) ;\left({ }^{7} B_{1 g}\right) ;\left({ }^{7} B_{2 g}\right)$; and $2\left({ }^{7} E_{g}\right)$, a total of $(2 S+1)(2 L+1)=49$ states. The introduction of the spin-orbit coupling will now cause each of these to be split further. Consider the level $\left({ }^{7} A_{2 g}\right)$. The rep $A_{2 g}$ in $D_{4 h}$ has the character $(1,1,1,-1,-1,1,1,1,-1,-1)$, while the representation from $D_{3}$ has the character (parity even, since it is a spin representation) $(7,-1,-1$, $-1,-1,7,-1,-1,-1,-1)$, so the representation $A_{23} \times D_{3}$ has the character $(7,-1,-1,1,1$, $7,-1,-1,1,1)$ and is reducible in to $A_{1 g}: B_{1 g}$; $B_{2 g}$; and $2 E_{g}$. The final states may be designated $\left({ }^{7} A_{2 g}\right) A_{1 g} ; \quad\left({ }^{7} A_{2 g}\right) B_{1 g} ; \quad\left({ }^{7} A_{2 g}\right) B_{2 g} ;$ and $2\left({ }^{7} A_{2 g}\right) E_{g}$. This notation parallels that of the usual $L-S$ coupling scheme. The labels inside the parentheses indicate the approximate transformation properties of the state for transformations of spin and orbit separately which leave invariant the Hamiltonian, while the final designation is the accurate specification of the behavior of the state under the simultaneous transformation of spin and orbit.

In the strong field case, we introduce the crystal field in our sequence of approximations (section 3.3) before considering the details of the electrostatic interaction of the electrons, retaining only the initial central field average $V^{\prime}\left(r_{i}\right)$. We now specify our initial energy by giving only the configuration, and the reduction of the representations begins at this point. As a matter of notation, following Mulliken (1955), we shall designate the reps and characters for a single electron by lower case letters (e.g., $a_{2 g}$ ). If there is but one electron, there is no distinction between the intermediate field and strong field cases, and we proceed as outlined for that case.

We shall illustrate the procedure for the case of
2 nowequivalent electrons, and then consider the restrictions imposed on equivalent electrons by the operation of the Pauli principle. We shall take the configuration $p p$ in a field of $\mathrm{C}_{2 v}$ symmetry. The threefold degeneracy of a $p$ state (apart from spin) is completely lifted, and we obtain (table 9 for $1_{u}$ ) $a_{1} ; b_{1}$; and $b_{2}$ for each electron, so the configuration breaks up into $\left(a_{1}\right)\left(a_{1}\right) ;\left(a_{1}\right)\left(b_{2}\right) ;\left(b_{2}\right)$ $\left(a_{1}\right)$; etc., a total of 9 possibilities corresponding to the original 9 orbital possibilities in $p p$. The spin transformation will be $D_{1 / 2} \times D_{1 / 2}$ : The introduction of the details of electrostatic interaction cannot cause any further splitting of the orbital wave function, since all the states are one dimensional, though a relabeling may occur. The requirement for totally antisymmetric wave functions will require that the spin transformation $D_{1 / 2} \times D_{1 / 2}$ be reduced to $D_{0}$ and $D_{1}$, giving the usual singlet and triplet states, respectively. Thus, $\left(a_{1}\right)$ $\left(a_{1}\right)$ becomes $\left(a_{1}\right)\left(a_{1}\right)\left({ }^{1} A_{1}\right)$ and $\left(a_{1}\right)\left(a_{1}\right)\left({ }^{3} A_{1}\right) ;\left(b_{1}\right)$ $\left(b_{2}\right)$ becomes $\left(b_{1}\right)\left(b_{2}\right)\left({ }^{1} A_{2}\right)$ and $\left(b_{1}\right)\left(b_{2}\right)\left({ }^{3} A_{2}\right)$; etc. Note that this latter state is distinct from $\left(b_{2}\right)$ $\left(b_{1}\right)\left({ }^{3} A_{2}\right)$, which is also an allowed state in the reduction. The further introduction of spin-orbit interaction now requires the reduction of $X_{i} \times D_{0}$ for the singlet states, $X_{i} \times D_{1}$ for the triplet states, in accordance with the overall symmetry of the problem, which is still that of the external crystal field potential $V$. Consider $\left(b_{1}\right)\left(b_{2}\right)\left({ }^{3} A_{2}\right)$. The character of $A_{2}$ is $(1,1,-1,-1)$, while the character of $D_{1}$ in $C_{2 v}$ (even parity for a spin transformation) is (from table 7) $(3,-1,-1,-1)$, so the character to be reduced is $(3,-1,1,1)$, giving $A_{1}, B_{1}$, and $B_{2}$. We may write the final states, then, as $p p\left(b_{1}\right)\left(b_{2}\right)\left({ }^{3} A_{2}\right) A_{1}$, or $p p\left(b_{1}\right)\left(b_{2}\right)\left({ }^{3} A_{2}\right) B_{1}$, for example.

If the electrons are equivalent, i.e., $p^{2}$, we write $\left(a_{1}\right)^{2}$ instead of $\left(a_{1}\right)\left(a_{1}\right)$, and we cannot distinguish $\left(a_{1}\right)\left(b_{2}\right)$ from $\left(b_{2}\right)\left(a_{1}\right)$. Furthermore, the Pauli principle requires the spin state of opposing spins for the orbital states $\left(a_{1}\right)^{2} ;\left(b_{1}\right)^{2} ;$ and $\left(b_{2}\right)^{2}$, so that these appear as singlets only. The remainder of the orbital states may appear either as singlets or as triplets. Apart from these considerations, the development follows that of the nonequivalent electrons. A possible final state would be written $p^{2}\left(b_{1}\right)^{2}\left(A_{1}\right) A_{1}$.

We have selected here a particularly simple case in that there is no orbital degeneracy present in the reduction of $D_{1}$ for a $p$ electron. The requirements of the Pauli principle are then particularly easy to satisfy. If the reduction yields a twodimensional rep, or remains three dimensional, the development must be followed more carefully. We introduce the procedure by considering, in the concepts of the present work, the familiar operation of the Pauli principle in labeling the levels of the configuration $p^{2}$ itself. We imagine a uniform magnetic field such that the resulting perturbation in the Hamiltonian, - M. $\mathbf{B}$, has the symmetry $C_{\infty h}$. The degeneracy of each $p$ electron is lifted, and we have (table 9) the states $a_{u}, c_{1 u}$ and $c_{-1 u}$ for each electron. Because of the Pauli 
principle, the state $\left(c_{1 u}\right)^{2} C_{2 \sigma}$ occurs with antiparallel spins only; i.e., a singlet state. We see that no state $C_{n \sigma}$ for $n$ greater than 2 arises. We then infer from table 9 that there exists a singlet level $L=2$ in the configuration when the magnetic field is removed. Similarly, we deduce a triplet $L=1$ and a singlet $L=0$.

A similar process can be used if the crystal field leaves degeneracy in the 1-electron wave functions. The differences are two: first, it is generally more convenient to remove the degeneracy by the imaginary application of an electrostatic potential of a symmetry which is a subgroup of that actually present; second, the correlation of states in this additionally perturbed problem with those of the primary perturbation is not unique, and several alternatives must be considered.

Let us further consider $p^{2}$, this time under the symmetry $\mathrm{C}_{4 v}$, where $1_{u}$ reduces to $a_{1}$ and $e$. Our problem really lies in the study of $(e)^{2}$, since $\left(a_{1}\right)(e)$ can yield either triplet or singlet, and $\left(a_{1}\right)^{2}$ is necessarily a singlet. Without the considerations of the Pauli principle, $(e)^{2}$ yields $A_{1}$, $A_{2}, B_{1}$, and $B_{2}$, both triplet and singlet, and the Pauli principle will suppress certain of these terms. Since (e), including spin, represents 4 states, we expect $\frac{4 \cdot 3}{2}=6$ states altogether. Let us reduce the symmetry to $\mathrm{C}_{2 v} ;(e)$ becomes $b_{1}+b_{2}$, and the allowed orbital states are $\left(b_{1}\right)^{2} ;\left(b_{2}\right)^{2}$; and $\left(b_{1}\right)\left(b_{2}\right)$. The first two are both $A_{1}$ and necessarily singlet, while the third is $A_{2}$, either singlet or triplet, so we have our total of six states. If we examine table 10 for the relations between these states of $\mathrm{C}_{2 v}$ and states of $\mathrm{C}_{4 v}$, we see that $A_{1}$ in the former implies either $A_{1}$ or $B_{1}$ in the latter. Since we have $A_{1}$ twice in $C_{20}$ and cannot have either $A_{1}$ or $B_{1}$ more than once from $(e)^{2}$ in $\mathrm{C}_{4 v}$, we conclude that both are present in $\mathrm{C}_{40}$ as singlet terms. However, the singlet, or the triplet, $A_{2}$ implies either $A_{2}$ or $B_{2}$, and we cannot decide which is the correct choice. If we reduce the symmetry to $\mathrm{C}_{4}$ instead of $\mathrm{C}_{2 v},(e)$ reduces to $\left(c_{1}\right)$ and $\left(c_{3}\right)$, yielding singlets $\left(c_{1}\right)^{2}$ and $\left(c_{3}\right)^{2}$, and singlet and triplet $\left(c_{1}\right)\left(c_{3}\right)$. The first two give $B$ states, while the third gives $A$ states. Examination of table 10 shows that $B$ in $C_{4}$ implies either $B_{1}$ or $B_{2}$ in $C_{40}$. Again, since we have two of the former, and are allowed at most one of each of the latter, we conclude the presence of singlet $B_{1}$ and singlet $B_{2}$, together with the singlet $A_{1}$ previously deduced. The triplet state in $C_{20}$ implies either $A_{2}$ or $B_{2}$ in $\mathrm{C}_{40}$, while the triplet state in $\mathrm{C}_{4}$ implies either $A_{1}$ or $A_{2}$. Obviously, the only choice consistent with both is the triplet $A_{2}$. We have, thus, ${ }^{1} A_{1} ;{ }^{3} A_{2}$; ${ }^{1} B_{1}$; and ${ }^{1} B_{2}$. The balance of the problem then follows previous discussion. In general, each problem must be considered as an individual case.

\subsection{Kramers Degeneracy}

There is one very important symmetry operation, in addition to spatial rotations and the inversion, which should be considered at this time. This is the symmetry of "time-reversal". This was initially considered by Kramers (1929, 1930) in connection with the rotation of the plane of polarization of polarized light. The matter has been thoroughly considered by Wigner (1932) in the nonrelativistic case in which we are here interested, and more recently by Klein (1952). We shall outline here the principal concepts involved, referring the reader to the original papers for additional details.

Classically, the reversal of time in a mechanical system is accomplished by the reversal of the direction of all velocities (or momenta) by the substitution $\mathbf{r}^{\prime}=\mathbf{r}, \mathbf{p}^{\prime}=-\mathbf{p}$. Quantum mechanically, we assume that this is also time reversal, if a similar substitution $s^{\prime}=-s$ is made for any spin angular momentum involved. In the case of a free atom, the Hamiltonian $\mathbf{H}(\mathbf{r}, \mathbf{p}, \mathbf{s})$ involves $\mathbf{p}$ and $\mathbf{s}$ in kinetic energy terms with $\mathbf{p}^{2}$ and spin-orbit interaction terms with $\mathbf{p} \cdot \mathbf{s}$, both of which are invariant under the time-reversal operation. This invariance of the Hamiltonian remains under the influence of external perturbations of a purely electrostatic character, in which case the vector potential A may be set equal to zero and the scalar potential $V(\mathbf{r})$ introduced. This invariance is destroyed by the application of magnetic fieldsa term $\mathbf{p} \cdot \mathbf{A}$ arises in the Hamiltonian, which is linear in $\mathbf{p}$ and changes sign on time reversal.

In general, then, we shall seek an operator $\mathbf{K}$ such that $\mathbb{K} \mathbb{H}(\mathbf{r}, \mathbf{p}, \mathbf{s}) \mathbf{K}^{-1}=\mathbf{H}(\mathbf{r},-\mathbf{p},-\mathbf{s})$, and $\mathbb{K} \Psi$ $(\mathbf{r}, s, t)=\Psi(\mathbb{r},-\varepsilon,-t)$. Since the operator $\mathbb{K}$ represents a transformation of our wave functions, we shall require it to be a unitary operator. If we consider the spin-free theory, with $\Psi(\mathbf{r}, t)=\psi(\mathbf{r}) e^{-\frac{i E t}{\hbar}}$, we see that the operation $t \rightarrow-t$ is accomplished merely by taking the complex conjugate, an operation we shall call $\mathbb{K}_{0}$. Insofar as the spin-free theory is concerned, this is sufficient; the p operator $-i \hbar \nabla$ becomes $-\mathbf{p}=+i \hbar \nabla$ upon taking the complex conjugate. Let us now consider the Pauli spin theory for one electron. The Pauli spin matrices

$\mathbf{s}_{x}=\left(\begin{array}{ll}0 & 1 \\ 1 & 0\end{array}\right) \quad \mathbf{s}_{y}=\left(\begin{array}{rr}0 & -i \\ i & 0\end{array}\right) \quad \mathbf{s}_{2}=\left(\begin{array}{rr}1 & 0 \\ 0 & -1\end{array}\right)$

are both unitary and Hermitian. The transformation $\mathbf{K}_{0} \mathbf{s}_{y} \mathbf{K}_{0}{ }^{-1}=-\mathbf{s}_{y}$ since $\mathbf{s}_{y}$ is purely imaginary, but $\mathbf{s}_{x}$ and $\mathbf{s}_{2}$, both real, are invariant under $\mathbf{K}_{0}$. Hence, in order to effect the reversal of $\mathbf{s}_{x}$ and $\mathbf{s}_{z}$, we seek yet another operator, which must also be a unitary operator $\mathbb{U}$, such that it commutes with $\mathbf{r}, \mathbf{p}, \mathbf{s}_{y}$, and $\mathbf{K}_{0}$, but anticommutes with $\mathbf{s}_{x}$ and $\mathbf{s}_{2}$ so that $\mathbf{U} \mathbf{s}_{x} \mathbf{U}^{-1}=-\mathbf{s}_{x}$ and similarly for $\mathbf{s}_{z}$. This latter requirement is met by $\mathbf{s}_{y}$ itself, or by some product of $\mathbf{s}_{y}$ with a complex number $a$ of modulus 1 . To ensure commutation with $\mathbf{K}_{0}$, the product $a \mathbf{s}_{y}$ must be real, which follows if $a=i$, so that $\mathbf{U}=i \mathbf{s}_{y}$ and $\mathbf{K}=\mathbf{K}_{0} i \mathbf{s}_{y}$. Note that the operator $\mathbf{U}$ is still unitary but no 
longer Hermitian. For $n$ electrons,

$$
\mathrm{U}=(i)^{n}\left(\mathbf{s}_{y}\right)_{1}\left(\mathbf{s}_{y}\right)_{2}\left(\mathbf{s}_{y}\right)_{3} \ldots
$$

Physically, it is clear that time reversal and any rotation-inversion operation commute. Mathematically, the inclusion of the operator $\mathbf{K}$ in an extended group of the form $D_{J} \times I \times K$ cannot be accomplished since $\mathbf{K}$ is not a linear operator. The basic rules for the operator $\mathbf{K}$ were given by Wigner as:

1. $\mathbf{K}^{2} \psi=\mathbf{U K}_{0} \mathbf{U K}_{0} \psi=\mathbf{U}^{2} \psi=(-1)^{n} \psi$, since $\mathbf{U}$ is real, $\mathbf{K}_{0}^{2}=1$, and $\mathbf{s}_{y}^{2}=\mathbf{1}$.

2. $\mathbf{K}(a \psi+b \phi)=a^{*} \mathbf{K} \psi+b * \mathbf{K} \phi$. This shows the nonlinearity of $\mathbf{K}$.

3. $(\phi, \psi)=\left(\mathbf{K}_{0} \psi, \mathbf{K}_{0} \phi\right)=\left(\mathbf{U K}_{0} \psi, \mathbf{U K}_{0} \phi\right)=(\mathbf{K} \psi, \mathbf{K} \phi)$. Applying (3) and (1), we have $(\mathbf{K} \psi, \psi)=(\mathbf{K} \psi$, $\mathbf{K} \mathbf{K} \psi)=\left(\mathbf{K} \psi, \mathbf{K}^{2} \psi\right)=(-1)^{n}(\mathbf{K} \psi, \psi)$. We see that the Hermitian scalar product must be zero if $n$ is odd, or that $\psi$ and $\mathbf{K} \psi$ are then orthogonal and hence linearly independent, yet have the same energy if $\mathbf{K H}=\mathbf{H K}$. This is the content of Kramers Theorem as usually stated, namely, that every level for an odd-electron system is at least twofold degenerate under the influence of external perturbations of a purely electrostatic character. We must examine further the inclusion of the operator $\mathbf{K}$ into our previous considerations on group representations.

Tie shall consider the following three cases (Frobenius and Schur, 1906; Wigner 1932):

1. The rep $R$ is real, or may be put in to some equivalent form which is wholly real. This refers to the elements $\mathbf{R}$ of the rep not merely the traces.

2. $R$ cannot be made real, and $R^{*}$ is not equivalent to it.

3. $R$ cannot be made real, but $R^{*}$ is equivalent to $R$.

For the finite groups a calculation of $\frac{1}{g} \sum_{\mathbf{R}} \chi\left(\mathbf{R}^{2}\right)$ will yield 1,0 , or -1 in cases (1), (2), or (3), respectively. Case (1) occurs for integral $J$ in $D_{J}$ and in all its subgroups save those one-dimensional reps where complex numbers appear explicitly in the character table (table 8). The only such specific reps of the double groups are $S_{3 g}, S_{3 u}$ of II $S_{6}$ and $S_{3}$ of ${ }_{1 I} C_{3}$. Case (2) holds in those onedimensional reps where there are obviously complex characters. The only two-dimensional reps in this category are the pairs $\left({ }_{2} S_{1 g, 2} S_{2 g}\right),\left({ }_{2} S_{1 u}\right.$, $\left.{ }_{2} S_{2 u}\right)$, and $\left({ }_{2} S_{1,2} S_{2}\right)$ in the groups ${ }_{\text {II }} \mathrm{T}_{h}$ and ${ }_{\text {II }} \mathrm{T}$. Case (3) does not hold for any reps of the single groups, but holds for all those of the double groups not in (1) or (2).

In case (1) let us consider a group operation $\mathbf{Q}$ such that $\mathbf{Q} \psi_{i}=\Sigma Q_{i j} \psi_{j}$ and $\mathrm{KQ} \psi_{i}=\mathbf{Q} \mathbf{K} \psi_{i}=\Sigma Q_{i j} \mathbf{K} \psi_{j}$, so the function $\mathbf{K} \psi$ will transform like $\psi$ under the operations of the group. Let us further consider $\phi_{i}=\psi_{i}+\mathbf{K} \psi_{i}$ and $\Omega_{i}=i\left(\psi_{i}-\mathbf{K} \psi_{i}\right)$. Clearly $\phi_{i}$ and $\Omega_{i}$ transform only among themselves under the group operations. Furthermore, if there is an even number of electrons, $\mathbf{K} \phi_{i}=\mathbf{K} \psi_{i}+\mathbf{K}^{2} \psi_{i}=\mathbf{K} \psi_{i}$ $+\psi_{i}=\phi_{i}$ and $\mathbf{K} \Omega_{i}=\mathbf{K} i \psi_{i}-\mathbf{K} i \mathbf{K} \psi_{i}=-i \mathbf{K} \psi_{i}+i \psi_{i}$ $=\Omega_{i}$ so the two sets of functions $\phi_{i}$ and $\Omega_{i}$ are not related by time reversal or otherwise, and any degeneracy present is presumably only an accidental degeneracy. Practically speaking, this means that our set of wave functions may be chosen to be real, and that in such a case time reversal yields no connections among the set of real functions that are not already present as a result of the rotation-inversion symmetry. If there is an odd number of electrons, $\mathbb{K} \phi_{i}=\mathbf{K} \psi_{i}-$ $\psi_{i} \neq \phi_{i}$ and we cannot so separate the $\phi$ and $\Omega$ sets of wave functions as we did above. Hence the rep must appear twice, once for $\psi$ and once for $\mathbf{K} \psi$ orthogonal to it. The only actual such cases are the reps $S_{3 g}, S_{3 u}$ of $\mathrm{S}_{6}$ and $S_{3}$ of $\mathrm{C}_{3}$ and it may be verified from table 9 for $S_{6}$ that they do in fact appear only an even number of times in any reduction of a group of half-integral $J$.

In case (2), $\mathbf{Q} \psi_{i}=\Sigma Q_{i j} \psi_{\text {, yields }} \mathbf{K} \mathbf{Q} \psi_{i}=\mathbf{Q} \mathbf{K} \psi_{i}$ $=\Sigma Q_{i}^{*} \mathbf{K} \psi_{j}$. Since the rep $R^{*}$ is not equivalent to $R$, $\psi$ and $\mathbf{K} \psi$ belong to different reps of the group and hence are orthogonal (section 4.3), but have the same energy. Hence a separation of a rep such as $E$ of $C_{3 v}$ into $C_{1}$ and $C_{2}$ of $\mathrm{C}_{3}$ by an electrostatic field does not imply a removal of the degeneracy. It is complex conjugate reps such as these which are usually grouped together into a single twodimensional rep by workers in the field of molecular spectroscopy.

The equivalence of $R$ and $R *$ in case (3) requires the existence of a unitary matrix $S$ such that $\mathrm{SRS}^{-1}=\mathbf{R}^{*}$ or $\mathbf{S}^{*} \mathbf{R}^{*}\left(\mathbf{S}^{-1}\right)^{*}=\mathbf{R}$, so $\mathbf{S}^{*} \mathbf{S R S}^{-1}\left(\mathbf{S}^{-1}\right)^{*}$ $=\mathbf{R}$ or $\mathbf{S} * \mathbf{S R}=\mathbf{R} \mathbf{S}^{*} \mathbf{S}$. We see that $\mathbf{S}^{*} \mathbf{S}$ commutes with every $\mathbf{R}$ and hence must be a multiple of the unit matrix, say $c \mathbb{E}$, with $c$ of absolute value 1 . With $\mathbf{S} * \mathbf{S}=c \mathbf{E}$, we have $\mathbf{S}^{*}=$ $c \mathbf{S}^{-1}=c(\tilde{\mathbf{S}})^{*}$, where $\tilde{\mathbf{S}}$ is the transposed matrix of S. This is a consequence of the unitary property of $\mathbf{S}$. The conjugate of this relation is $\mathbf{S}=c^{*} \tilde{\mathbf{S}}$, and the transpose of this gives $\widetilde{\mathbf{S}}=c^{*} \mathbf{S}$, finally giving $\mathbf{S}=c^{*}\left(c^{*}\right) \mathbf{S}$, so $\left(c^{*}\right)^{2}=1$ or $c= \pm 1$. If $c=+1$, we have $S$ with +1 along the minor diagonal and zero elsewhere - in this case $\mathbf{R}=\mathbb{R}^{*}$ and case (1) applies, so we take $c=-1$. Then $\mathbf{S}$ will consist of alternate +1 and -1 along the minor (nonprincipal) diagonal. Incidentally, this requires that the dimensionality of $\mathbf{S}$ be even.

Let us put $\mathbf{S}$ in the form such that +1 is in the upper right corner, and let us label the rows and columns of $\mathbf{S}$ and the $\mathbf{R}$ matrices by the numbers $j, j-1, j-2, \ldots-j$, where $j$ is half-integral.

Then

$S_{p q}=(-1)^{j-p} \delta_{p(-q)}$ and $\left(S^{-1}\right)_{a b}=(-1)^{j-a+1} \delta_{a(-b)}$.

The transformation $\mathrm{SRS}^{-1}$ is then as follows:

$$
\begin{aligned}
\left(R S^{-1}\right)_{a b}=\sum_{k} R_{a k}\left(S^{-1}\right)_{k b}=\sum_{k} R_{a k} & (-1)^{j-k+1} \delta_{k(-b)} \\
& =(-1)^{j+b+1} R_{a(-b)}
\end{aligned}
$$


and

$$
\begin{aligned}
\left(\operatorname{SRS}^{-1}\right)_{c d} & =\sum_{k}(-1)^{j-c} \delta_{c(-k)}(-1)^{j+d+1} R_{k(-d)} \\
& =(-1)^{2 j+1+d-c} R_{(-c)(-d)}=(-1)^{d-c} R_{(-c)(-d)} .
\end{aligned}
$$

This latter term must then also be $\left(R^{*}\right)_{c d}$, since $\mathbf{R}$ and $\mathbf{R}^{*}$ are equivalent by the transformation $\mathbf{S}$.

We now consider

and

$$
Q \psi_{a}=\sum_{b} Q_{a b} \psi_{b}
$$

$\mathrm{KQ} \psi_{a}=\mathrm{QK} \psi_{a}$

$$
=\sum_{b} Q^{*}{ }_{a b} \mathbf{K} \psi_{b}=\sum_{b}(-1)^{b-a} Q_{(-a)(-b)} \mathbf{K} \psi_{b}
$$

or

$$
\mathbf{Q}(-1)^{a} \mathbf{K} \psi_{a}=\sum_{b} Q_{(-a)(-b)}(-1)^{b} \mathbf{K} \psi_{b}
$$

If we compare this with the transformation on $\psi_{-a}$ obtaining $\mathbf{Q} \psi_{-a}=\sum_{-b} Q_{(-a)(-b)} \psi_{-b}$ we see that $(-1)^{a} \mathbf{K} \psi_{a}$ will transform like $\psi_{-a}$ under the group operations, and that $(-1)^{-a} \mathbf{K} \psi_{-a}$ will transform like $\psi_{a}$. The linear combination $\phi_{a}=\psi_{a}+(-1)^{-a} \mathbf{K} \psi_{-a}$ will transform in a similar fashion under the group opera-tions. Further, we observe that

$$
\begin{aligned}
\mathbf{K} \phi_{a}=\mathbf{K} \psi_{a}+(-1)^{a} \mathbf{K}^{2} \psi_{-a}=\mathbf{K} \psi_{a}-(-1)^{a} \psi_{-a} \\
=-(-1)^{a}\left[\psi_{-a}+(-1)^{a} \mathbf{K} \psi_{a}\right]=-(-1)^{a} \phi_{-a},
\end{aligned}
$$

since our convention has " $a$ " half-integral, $(-1)^{a}$ is purely imaginary and $\mathbb{K}_{0}(-1)^{a}=(-1)^{-a}=$ $-(-1)^{a}$, and $\mathbb{K}^{2} \psi_{a}=-\psi_{a}$. The important point is that the set of functions $\phi_{a}(a=j, j-1, \ldots .-j)$ transform among themselves under the group operations, and that the operator $\mathbb{K}$ merely yields a wave function which, apart from a phase factor, is already in the set. Similarly, if $\Omega_{a}=i\left(\psi_{a}-(-1)^{a} \mathbf{K} \psi_{-a}\right)$ the $\Omega_{a}$ will also transform among themselves under the group operations and time reversal, but will not involve the $\phi_{a}$. Hence we conclude that the introduction of time reversal does not require any additional essential degeneracy in case (3).

For emphasis, we shall summarize the cases where we find that the symmetry of time reversal (with external electrostatic fields only) gives us degeneracy beyond that expected from previous considerations.

1. States belonging to complex conjugate reps are degenerate. This is the case for both an odd or even number of electrons.

2. The reps $S_{3 g}, S_{3 u}$ of $S_{6}$ and $S_{3}$ of $C_{3}$, arising from odd-electron systems will always occur twice, once for $\psi$ and once for its orthogonal but degenerate conjugate $\mathbf{K} \psi$.

Finally, let us consider

$$
\mathbf{K}=(i)^{n}\left(\mathbf{S}_{y}\right)_{1}\left(\mathbf{S}_{y}\right)_{2} \ldots .\left(\mathbf{S}_{y}\right)_{n} \mathbf{K}_{0}
$$

operating on a wave function consisting of $2^{n}$ terms of the form

$$
\psi=f(\mathbf{r}) \zeta_{1}\left(S_{1}\right) \zeta_{2}\left(S_{2}\right) \cdot . \quad \zeta_{n}\left(S_{n}\right)
$$

Since

$\mathbf{S}_{y} \zeta(S)=(-1)^{1 / 2-S} i \zeta(-S), i \mathbf{S}_{y} \zeta(S)=(-1)^{1 / 2+S} \zeta(-S)$

and

$$
\mathbf{K} \psi=f^{*}(\mathbf{r})(-1)^{\frac{n}{2}+\Sigma S_{i}} \zeta_{1}\left(-S_{1}\right) \zeta_{2}\left(-S_{2}\right) \ldots \zeta_{n}\left(-S_{n}\right)
$$

It will be noted that $\mathbf{K}$ will commute with $\mathbf{L}^{2}, \mathbf{S}^{2}$ and $\mathbf{J}^{2}$, but not with $\mathbf{L}_{z}, \mathbf{S}_{z}$ or $\mathbf{J}_{z}$. If $f(\mathbf{r})$ belongs to the eigenvalue $L$ and $M_{L}, f^{*}(\mathbf{r})$ will belong to $L,-M_{L}$ if the usual phase factor $(-1)^{M_{L}}$ is supplied. Similarly, the original spin terms, belonging to $S, M_{S}$ will belong to $S,-M_{S}$ with the phase factor $(-1)^{\frac{n}{2}+M_{S}}$. Thus

$$
\begin{aligned}
& \mathbf{K} \psi\left(L, M_{L}, S, M_{S}\right) \\
& \quad=(-1)^{M_{L}(-1)^{M_{S}}(-1)^{\frac{n}{2}} \psi\left(L,-M_{L}, S,-M_{S}\right) .}
\end{aligned}
$$

The transformation from $L, M_{L}, S, M_{S}$ to $L, S, J$, $M_{J}$ is accomplished by the Clebsch-Gordan coefficients (section 5.2), which have phase factors such that

$$
C_{M_{L}}^{L} \stackrel{S}{M_{S}}{ }^{J}=(-1)^{L+S-J} C_{-M_{L} M_{S}}^{L}{ }^{S}
$$

In a state described by $\mathcal{J}, M_{J}$, we must also introduce this factor, and we obtain finally

$$
\begin{aligned}
& \mathbf{K} \psi\left(L, S, J, M_{J}\right)
\end{aligned}
$$

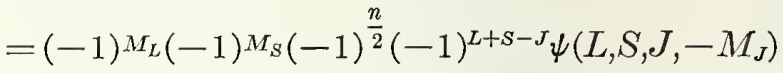

$$
\begin{aligned}
& =(-1)^{J-M_{J}(-1)^{L+S+\frac{n}{2}}} \psi\left(L, S, J,-M_{J}\right) \text {. }
\end{aligned}
$$

The factor $(-1)^{L+S+\frac{n}{2}}$ we shall neglect, but the

term $(-1)^{J-M_{J}}$ is of some importance, since we shall be considering states which are a linear combination of states with the same $J$ and different $M_{J}$. There will be a physically significant shift of relative phases among the components of such states when passing to the time-reversed state.

\subsection{Orthogonal States and Vanishing Matrix Elements}

Before proceeding to the consideration of selection rules and polarization, we shall digress here briefly to consider certain aspects of the calculation of matrix elements of the form $\int \psi_{b}^{*} X \psi_{a} d \tau$ which will be required at this time, deferring to the next section additional details. Let us assume initially that $X=1$. Let $\psi_{a}$ and $\psi_{b}$ belong to reps (1) and (2) respectively of the appropriate symmetry group, so that any group 
operation $\mathrm{Q}$ will yield $\mathrm{Q} \psi_{a}=\sum_{k} Q_{a k}^{(1)} \psi_{k}^{(1)}$ and $\mathrm{Q} \psi_{b}=\sum_{j} Q_{b i}^{(2)} \psi_{i}^{(2)}$, where $Q_{a k}^{(1)}$ and $Q_{b j}^{(2)}$ are the same if the two reps (1) and (2) are the same, otherwise they are distinct. The operation $\mathbf{Q}$ on $\psi_{b}^{*} \psi_{a}$ then gives $\sum_{j, k} Q_{b j}^{(2) *} Q_{a k}^{(1)} \psi_{j}^{(2) *} \psi_{k}^{(1)}=\sum_{j k} Q_{b a, j k} \psi_{j}^{(2) *} \psi_{k}^{(1)} \cdot$ The matrix with elements $Q_{b a, j k}$ is called the direct product of $\mathbf{Q}^{(2) *}$ and $\mathbf{Q}^{(1)}$, written $\mathbf{Q}^{(2) * \times(1)}$. If these latter are of dimensionality $n_{1}$ and $n_{2}$, then the direct product is a $n_{1} n_{2} \times n_{1} n_{2}$ matrix. Obviously, $Q^{(2) * \times(1)}$ is an element of a representation of the group-its trace is $\sum_{a b} Q_{b a, b a}=\left(\sum_{b} Q_{b b}^{(2) *}\right)\left(\sum_{a} Q_{a a}^{(1)}\right)$,

the product of the traces of the two "factor" matrices.

In general, $Q^{(2) * \times(1)}$ will be either a rep or a sum of reps, in which latter case it may be reduced by the methods of the preceding section. In either case, we obtain $\sum_{j, k} Q_{b a, j k} \psi_{i}^{(2) *} \psi_{k}^{(1)}=\sum_{d} Q_{c d}^{(3)} \psi_{d}^{(3)}+$ $\sum_{f} Q_{e f}^{(4)} \psi_{f}^{(4)} \ldots$ where $\psi_{d}^{(3)}, \psi_{f}^{(4)}$, etc., are the combinations of $\psi_{j}^{(2)} \psi_{k}^{(1)}$ appropriate to the reduced representation. Our original integral then becomes $\sum_{d} Q_{c d}^{(3)} \int \psi_{d}^{(3)} d \tau+\sum_{f} Q_{e f}^{(4)} \int \psi_{f}^{(4)} d \tau \ldots$

The basic argument we shall use here is that our integral, i.e., the matrix element, represents a physically significant quantity and hence cannot depend on the choice of any symmetrically equivalent reference frame. Consequently, the terms of the form $\sum Q_{c d} \int \psi_{d}^{(3)} d \tau$ must either actually be independent of $\mathbf{Q}$ or must vanish. If the rep (3) is actually independent of $\mathbf{Q}$, it is then in fact the completely symmetric or identity rep of the group, and we must ascertain the conditions under which the rep is present in the representation $Q^{(2) * \times(1)}$.

The identity rep has the character +1 for every group operation, so the orthogonality theorem for group characters tells us that $\frac{1}{g} \sum_{\mathbf{Q}} \sum_{a b} Q_{b a, b a}$ is the number of times the symmetric rep occurs in the reduction. But we recall that $\sum_{\mathbf{Q}} \sum_{a b} Q_{b a, b a}=$ $\sum_{Q}\left(\sum_{b} Q_{b b}^{(2) *}\right)\left(\sum_{a} Q_{a a}^{(1)}\right)$, and this latter expression is merely the orthogonality theorem applicd to the characters of the reps (1) and (2), which is zero unless $(1)=(2)$. Hence we conclude the very important result, that states belonging to different reps of the appropriate symmetry group are orthogonal.

The example just considered took $X=1$. If $X$ itself, either a function or operator, transforms as a rep or sum of reps of the symmetry group, the product $X \psi_{a}^{(1)}$ must first be reduced. If this reduction does not contain the rep (2), then the integral must vanish.

\subsection{Selection Rules and Polarization}

Selection rules and polarization rules are intimately connected, and we shall consider them together. We shall consider in detail the effect of an incident perturbing electromagnetic radiation superimposed upon the static electric and/or magnetic field already present. In a region of space not including the sources of the fieid, the perturbing radiation is usually described by its associated vector potential $\mathbf{A}$, with the auxiliary requirement $\boldsymbol{\nabla} \cdot \mathbf{A}=0$, which will allow us to set the scalar potential $V=0$. The fields $\mathbb{E}$ and $\mathbf{B}$ are then given by $\mathbf{E}=-\frac{1}{c} \frac{\partial \mathbf{A}}{\partial t}$ and $\mathbf{B}=\nabla \times \mathbf{A}$. This vector potential modifies the classical Hamiltonian term for one electron, $\frac{1}{2 m} \mathbf{p}^{2}$, to

$$
\frac{1}{2 m}\left(\mathbf{p}-\frac{e}{c} \mathbf{A}\right)^{2}=\frac{1}{2 m}\left(\mathbf{p}^{2}-\frac{e}{c} \mathbf{p} \cdot \mathbf{A}-\frac{e}{c} \mathbf{A} \cdot \mathbf{p}+\frac{e^{2}}{c^{2}} \mathbf{A}^{2}\right)
$$

Neglecting the term in $\mathrm{A}^{2}$, we obtain as the perturbation term

$$
\begin{aligned}
& \mathbf{H}^{\prime}=-\frac{e}{2 m c}(\mathbf{p} \cdot \mathbf{A}+\mathbf{A} \cdot \mathbf{p}) \\
&=-\frac{e}{2 m c}[\mathbf{A} \cdot \mathbf{p}+\mathbf{A} \cdot \mathbf{p}+(\mathbf{p} \cdot \mathbf{A})] .
\end{aligned}
$$

Since $\mathrm{p}$ is, apart from a constant factor, the vector operator $\nabla$, the term (p.A) vanishes and our final $\boldsymbol{H}^{\prime}=-\frac{e}{m c} \mathbf{A} \cdot \mathbf{p}$. This acts on each electron individually, and the total perturbation requires that this be added for all electrons in the atom. If a system is initially in a state $\Psi_{a}=\psi_{a} e^{-\frac{i E_{a} l}{\hbar}}$ the probability $C_{b} C_{b}^{*}$ that the system under the influence of the time-dependent perturbation $\mathbf{H}^{\prime}$ will at a later time be in the state $\Psi_{b}$ is determined by

$$
\frac{d C_{b}}{d t}=-\frac{i}{\hbar} \int \Psi_{b}^{*} \mathbf{H}^{\prime} \Psi_{a} d \tau
$$

so we shall investigate this integral.

The components of $\mathrm{A}$ are functions of $(x, y, z, t)$. We shall assume that the variation of $\mathrm{A}$ with $t$ is harmonic. We shall not require explicitly the harmonic factor $e^{i \omega t}$ in the following development and shall generally omit further reference to it; i.e., $\mathbf{A}(x, y, z, t)=\mathbf{A}(x, y, z) e^{i \omega t}$. For the wavelengths of light in which we arc interested, $\lambda$ is of the order of $10^{-4} \mathrm{~cm}$. In comparison with the order of size of the atom, $10^{-8} \mathrm{~cm}$, to a first approximation the variation of the components of $\mathbf{A}$ with $(x, y, z)$ may frequently be neglected. We shall see that this approximation may be inadequate for our purposes, and we will improve this approximation by developing the components of the vector po- 
tential in a Taylor's series about the origin. This will yield

$$
A_{x}(x, y, z)=A_{x}^{o}+x\left(\frac{\partial A_{x}}{\partial x}\right)_{0}+y\left(\frac{\partial A_{x}}{\partial y}\right)_{0}+z\left(\frac{\partial A_{x}}{\partial z}\right)_{0},
$$

where the derivatives are evaluated at the origin. Similar relations hold for $A_{y}$ and $A_{z}$. To this approximation, $\mathrm{H}^{\prime}$ becomes

$$
\begin{array}{r}
H^{\prime}=-\frac{e}{m c} \sum\left[\left(A_{x}^{0} p_{x}+A_{y}^{0} p_{y}+A_{z}^{0} p_{z}\right)+\left(\frac{\partial A_{x}}{\partial x}\right) x p_{x}\right. \\
+\left(\frac{\partial A_{x}}{\partial y}\right) y p_{x}+\left(\frac{\partial A_{x}}{\partial z}\right) z p_{x}+\left(\frac{\partial A_{y}}{\partial x}\right) x p_{y} \\
+\left(\frac{\partial A_{y}}{\partial y}\right) y p_{y}+\left(\frac{\partial A_{y}}{\partial z}\right) z p_{y}+\left(\frac{\partial A_{z}}{\partial x}\right) x p_{z} \\
\left.+\left(\frac{\partial A_{z}}{\partial y}\right) y p_{z}+\left(\frac{\partial A_{z}}{\partial z}\right) z p_{z}\right] .
\end{array}
$$

The first three of these terms will involve matrix elements of the components of $\mathbf{p}$ only, since $\mathbf{A}^{0}$ is a constant. The general quantum-mechanical equations of motion allow $\int \Psi_{b}^{*} \mathrm{p} \Psi_{a} d \tau$ to be written as $\frac{i m}{\hbar}\left(E_{b}-E_{a}\right) \int \psi_{b}^{*} \mathbf{r} \psi_{a} d \tau$. Note that our integral now involves the time-independent wave functions. Thus, these three terms can be written in terms of the components of the vector $r$ rather than of p. Since $e r$ is the classical electric dipole, transitions induced by these three terms are called electric dipole transitions. Let us assume temporarily that there is no external perturbation apart from the radiation, so that $J$ and parity remain good quantum numbers. Let us consider an initial state transforming as $J_{\sigma}, J \neq 0$. Each of the vector components $x, y$, and $z$ will transform as $1_{u}$ (i.e., as the components of an odd state $J=1$ ). Applying our previous considerations, the character of the rep $J_{g}$ is $\sum_{-J}^{J} e^{i M \phi}$ for both proper and improper rotations, while that for $1_{u}$ is $\left(e^{-i \phi}+1+e^{i \phi}\right)$ for proper rotations and $(-1)$ times this for improper rotations. The product of the characters for pure rotation yields

$$
\begin{aligned}
\left(e^{-i \phi}+1+e^{i \phi}\right)\left(\sum_{-J}^{J} e^{i M \phi}\right)= & \sum_{-(J+1)}^{J+1} e^{i M \phi} \\
& +\sum_{-J}^{J} e^{i M \phi}+\sum_{-(J-1)}^{J-1} e^{i M \phi},
\end{aligned}
$$

and $(-1)$ times this for the improper rotations. It is immediately clear that the direct product $1_{u} \times J_{g}$ yields $(J-1)_{u}+J_{u}+(J+1)_{u}$, and (from section 4.3) our final state arising from the transition must be one of these. This is equivalent to the well-known selection rule $\Delta J=0, \pm 1$, and a particular example of Laporte's rules, where even must combine with odd terms. If $J=0$, $1_{u} \times 0_{g}=1_{u}$, and we obtain the restriction that $J=0$ does not combine with $J=0$.

The other set of 9 terms in $\mathbf{H}^{\prime}$ does not itself transform under a single rep of the rotation group, and must be separated into terms, each one of which does so transform. The 9 terms consist of the general products of the 3 components of the vector $\mathbf{r}$ with those of the vector $\mathbf{p}$. It is well known that three linearly independent terms arise from the vector product $\mathbf{r} \times \mathbf{p}$, and a fourth arises from the scalar product $\mathbf{r} \cdot \mathbf{p}$, leaving five terms, constituting the components of an irreducible tensor of rank 2 (in contrast to the original set of 9 components, which is also a tensor but not irreducible). In the terminology of the preceding paragraph, we have $1_{u} \times 1_{u}=2_{g}+1_{g}+0_{g}$. In order to exhibit these more explicitly, let us consider $\quad\left(\frac{\partial A_{x}}{\partial y}\right) y p_{x}+\left(\frac{\partial A_{y}}{\partial x}\right) x p_{y^{*}} \quad$ By $\quad$ adding and subtracting $\left(\frac{\partial A_{x}}{\partial y}\right) x p_{y}+\left(\frac{\partial A_{y}}{\partial x}\right) y p_{x}$ the expression may be brought in to the form

$$
\begin{aligned}
\frac{1}{2}\left(\frac{\partial A_{x}}{\partial y}+\frac{\partial A_{y}}{\partial x}\right)\left(y p_{x}+\right. & \left.x p_{y}\right) \\
+ & \frac{1}{2}\left(\frac{\partial A_{y}}{\partial x}-\frac{\partial A_{x}}{\partial y}\right)\left(x p_{y}-y p_{x}\right)
\end{aligned}
$$

and a similar relation may be derived for the other 2 pairs of similar form. This process amounts to finding the antisymmetric portion of the original 9 components, viewed as a $3 \times 3$ matrix. We observe that the first factor of the second term is the $z$ component of $\nabla \times \mathbf{A}=\mathbf{B}$ and that the second factor is the $z$ component of $\mathbf{r} \times \mathbf{p}=\mathbf{L}$, so, including the other 2 pairs of terms, we have a contribution $-\frac{e}{2 m c} \mathbf{B} \cdot \mathbf{L}$ to $\mathbf{H}^{\prime}$. Since the quantity $-\frac{e}{2 m c} \mathbf{L}$ is the magnetic moment operator, transitions arising from these terms are called magnetic dipole transitions. The components of $\mathbf{L}$ (a pseudovector) transform as $1_{\theta}$ in contrast to the electric dipole's $1_{u}$, so here even states combine with even, or odd with odd. We may note at this point that we have not yet included any terms in our radiative perturbation representing the interaction of the radiation with the spin of the electron. This may be accomplished at this point (in a more or less ad hoc manner) by using $-\frac{e}{2 m c}(\mathbf{L}+2 \mathrm{~S})$ as the interaction with the magnetic part of the incident radiation, though this will not change the transformation properties of the term, since spin is also an angular momentum operator with the transformation $1_{g}$. It will be recalled from section 3.2 that the transformation (3.6) is equivalent to a rotation among the components of the spin angular momentum 
operator (then called $\mathbf{R}$ ) and hence equivalent to a transformation like the three components of a state $J=1$.

In considering the other six linearly independent terms, we again have recourse to the general quantum mechanical equations of motion. In particular, we obtain

$$
\int \Psi_{b}^{*}\left(x p_{x}\right) \Psi_{a} d \tau=\frac{i m}{\hbar}\left(E_{b}-E_{a}\right) \int \psi_{b}\left(\frac{1}{2} x^{2}\right) \psi_{a} d \tau
$$

and

$$
\int \Psi_{b}^{*}\left(x p_{y}+y p_{x}\right) \Psi_{a} d \tau=\frac{i m}{\hbar}\left(E_{b}-E_{a}\right) \int \psi_{b}(x y) \psi_{a} d \tau .
$$

If we further simplify the notation by writing $A_{x x}$ for $\left(\frac{\partial A_{x}}{\partial x}\right)$ and $A_{x y}$ for $\left(\frac{\partial A_{x}}{\partial y}+\frac{\partial A_{y}}{\partial x}\right)$ our remaining perturbation can be written in terms of the matrix elements of

$$
\left(A_{x x} x^{2}+A_{y y} y^{2}+A_{z z} z^{2}+A_{x y} x y+A_{x z} x z+A_{y z} y z\right) .
$$

In lieu of $x^{2}, y^{2}$, and $z^{2}$, let us consider the linearly independent combinations

$$
\begin{aligned}
& r^{2}=x^{2}+y^{2}+z^{2} \\
& Z^{2}=2 z^{2}-x^{2}-y^{2} \\
& \rho^{2}=x^{2}-y^{2}
\end{aligned}
$$

with the inverse relations

$$
\begin{aligned}
& x^{2}=\frac{1}{2} \rho^{2}+\frac{1}{3} r^{2}-\frac{1}{6} Z^{2} \\
& y^{2}=\frac{1}{3} r^{2}-\frac{1}{6} Z^{2}-\frac{1}{2} \rho^{2} \\
& z^{2}=\frac{1}{3} Z^{2}+\frac{1}{3} r^{2} .
\end{aligned}
$$

Substituting, we have as our perturbation the matrix elements of

$$
\begin{aligned}
r^{2}\left(\frac{1}{3} A_{x x}+\right. & \left.\frac{1}{3} A_{y y}+\frac{1}{3} A_{z z}\right)+Z^{2}\left(\frac{1}{3} A_{z z}-\frac{1}{6} A_{x x}-\frac{1}{6} A_{y y}\right) \\
& +\rho^{2}\left(\frac{1}{2} A_{x x}-\frac{1}{2} A_{y y}\right)+A_{x y} x y+A_{x z} x z+A_{y z} y z .
\end{aligned}
$$

Of these six terms, that in $r^{2}$ is the $O_{g}$ term, invariant under rotation and inversion, and here actually zero, since $A_{x x}+A_{y y}+A_{z z}=\operatorname{div} \quad \mathbf{A}=0$. Notice that the terms $Z^{2}, x y$, etc., are, apart from normalization factors, the tesseral harmonics of degree 2 (table 2).

The remaining five terms are the components of the quadrupole moment, and transitions arising from them are called electric quadrupole transitions. The general selection rule is here given by a consideration of the $\mathscr{Q}_{g}$ transformation properties of the quadrupole moment. If $J \geq 2$, $\mathscr{2}_{g} \times J_{g}=(J+2)_{g}+(J+1)_{g}+J_{g}+(J-1)_{g}+(J-2)_{g}$, so the general selection rule is $\Delta J= \pm 2, \pm 1,0$. If $J=1,2_{g} \times 1_{o}=3_{g}+2_{o}+1_{\theta}$, so $J=1$ does not combine with $J=0$. Likewise, $\mathscr{2}_{g} \times 0_{g}=\mathscr{2}_{g}$, so $J=0$ does not combine with $J=0$ or $J=1$.

We have up to this point considered that the symmetry present was that of the full three-dimensional rotation-inversion group. If this is not the case, the 3 electric dipole components, the 3 magnetic dipole components, and the 5 electric quadrupole components will belong to different reps of the symmetry group. These may be determined from table 9 for $1_{u}, 1_{g}$, and $\mathscr{2}_{g}$, respectively, and the detailed correlation may in most cases be obtained from table 10. The use of table 10 after an initial application of table 9 for the group $\mathrm{D}_{\infty h}$ (for noncubic fields) is perhaps most convenient. The reduction to $\mathrm{D}_{\infty h}$ will separate both $1_{u}$ and $1_{g}$ into one 1-dimensional rep and one 2 -dimensional rep, while $\mathscr{2}_{\theta}$ yields one 1-dimensional rep and two 2-dimensional reps. These may be followed to lower symmetries with table 10 . Details may be obtained from table 8 . For example, $\mathrm{L}$, belonging to $1_{\theta}$, yields, in $\mathrm{D}_{\infty h}, L_{2}$ belonging to $A_{2 g}$, while $L_{x}$ and $L_{y}$ both belong to $E_{1 g}$. Upon reducing the symmetry to $\mathrm{C}_{4 v}, L_{z}$ now becomes $A_{2}$ while $L_{x}$ and $L_{y}$ now both belong to $E$. If we consider the reduction from $\mathrm{D}_{\infty h}$ to $C_{\infty h}$, we see that $E_{1 g}$ splits into $C_{1 g}$ and $C_{-1 g}$. However, these do not represent the transformation of either $L_{x}$ or $L_{y}$, but of $L_{x}+i L_{y}$ and $L_{x}-i L_{y}$, respectively. In this case, and also for the subgroups of $\mathrm{C}_{\infty}$, we shall consider these latter, along with $L_{z}$, as the three linearly independent components, each of which involves only one rep of the symmetry group. Similarly, we shall resolve the electric dipole term $\mathrm{r}$ into $x, y$, and $z$ for most groups, using $x+i y, x-i y$, and $z$ for the subgroups of $\mathrm{C}_{\infty h}$, and the electric quadrupole components into $Z^{2}, x z, y z, \rho^{2}, x y$, or, alternatively, $Z^{2}, z(x+i y)$, $z(x-i y),(x+i y)^{2}$, and $(x-i y)^{2}$. These resolutions are presented in table 12 .

As usual, there are two possibilities for the cubic groups, one in which the $z$-axis is an even-fold axis and one in which it is an odd-fold axis. This makes a difference only for the quadrupole components, since the electric and magnetic dipole components remain indistinguishable under these symmetries. If we consider the $z$-axis to be an even-fold axis, i.e., $\mathrm{C}_{4}$ for $\mathrm{O}$ and $\mathrm{O}_{h}, \mathrm{~S}_{4}$ for $\mathrm{T}_{d}, \mathrm{C}_{2}$ for $T_{h}$ and $T$, the five real quadrupole components remain a satisfactory basis for the resolution in all cases except for two components in $T_{h}$ and $T$, where we require the normalized components

$$
\frac{1}{\sqrt{2}}\left[C_{2}^{0}+i C_{2}^{2}\right] \quad \text { and } \quad \frac{1}{\sqrt{2}}\left[C_{2}^{0}-i C_{2}^{2}\right]
$$

transforming as $C_{1 g}$ and $C_{2 g}$ respectively.

If we take the $z$-axis to be the $\mathrm{C}_{3}$ axis in these cases, we find that these quadrupole components, the tesseral harmonics of degree 2, do not serve as a basis for the reduction of the $\mathscr{2}_{g}$ representation 
TABLE 12. Multipole moment transformations

\begin{tabular}{|c|c|c|c|c|c|c|c|c|c|c|c|}
\hline & $x$ & $y$ & $z$ & $L_{x}$ & $L_{y}$ & $L_{z}$ & $Z^{2}$ & $x z$ & $y z$ & $\rho^{2}$ & $x y$ \\
\hline $\begin{array}{l}\mathrm{D}_{\infty h} \\
\mathrm{C}_{\infty \downarrow}\end{array}$ & $\begin{array}{l}E_{1 u} \\
E_{1}\end{array}$ & $\begin{array}{l}E_{1 u} \\
E_{1}\end{array}$ & $\begin{array}{l}A_{2 u} \\
A_{1}\end{array}$ & $\begin{array}{l}E_{1 g} \\
E_{1}\end{array}$ & $\begin{array}{l}E_{1 \mathrm{~g}} \\
E_{1}\end{array}$ & $\begin{array}{l}A_{2 g} \\
A_{2}\end{array}$ & $\begin{array}{l}A_{1 g} \\
A_{1}\end{array}$ & $\begin{array}{l}E_{1 g} \\
E_{1}\end{array}$ & $\begin{array}{l}E_{1 g} \\
E_{1}\end{array}$ & $\begin{array}{l}E_{2 g} \\
E_{2}\end{array}$ & $\begin{array}{l}E_{2 g} \\
E_{2}\end{array}$ \\
\hline $\begin{array}{l}\mathrm{O}_{h} \\
\mathrm{~T}_{d}\end{array}$ & $\begin{array}{l}T_{1 u} \\
T_{2}\end{array}$ & $\begin{array}{l}T_{1 u} \\
T_{2}\end{array}$ & $\begin{array}{l}T_{1 u} \\
T_{2}\end{array}$ & $\begin{array}{l}T_{1 \mathrm{~g}} \\
T_{1}\end{array}$ & $\begin{array}{l}T_{1 g} \\
T_{1}\end{array}$ & $\begin{array}{l}T_{1 g} \\
T_{1}\end{array}$ & $\begin{array}{l}E_{g} \\
E\end{array}$ & $\begin{array}{l}T_{2 g} \\
T_{2}\end{array}$ & $\begin{array}{l}T_{2 g} \\
T_{2}\end{array}$ & $\stackrel{E}{E}_{E}$ & $\begin{array}{l}T_{2 g} \\
T_{2}\end{array}$ \\
\hline $\begin{array}{l}\mathrm{T}_{h} \\
\mathrm{D}_{6 h}\end{array}$ & $\begin{array}{l}T_{u} \\
E_{1 u}\end{array}$ & $\begin{array}{l}T_{u} \\
E_{1 u}\end{array}$ & $\begin{array}{l}T_{u} \\
A_{2 u}\end{array}$ & $\begin{array}{l}T_{g} \\
E_{1 \mathrm{~g}}\end{array}$ & $\begin{array}{c}T_{g} \\
E_{1 g}\end{array}$ & $\begin{array}{l}T_{g} \\
A_{2 g}\end{array}$ & $A_{1 g}^{(\mathrm{a})}$ & $\begin{array}{c}T_{g} \\
E_{1 g}\end{array}$ & $\begin{array}{c}T_{g} \\
E_{1 g}\end{array}$ & $E_{2 g}^{(\mathrm{a})}$ & $\begin{array}{l}T_{g} \\
E_{2 g}\end{array}$ \\
\hline $\begin{array}{l}\mathrm{D}_{4 h} \\
\mathrm{C}_{60}\end{array}$ & $\begin{array}{l}E_{u} \\
E_{1}\end{array}$ & $\begin{array}{l}E_{u} \\
E_{1}\end{array}$ & $\begin{array}{l}A_{2 u} \\
A_{1}\end{array}$ & $\begin{array}{l}E_{8} \\
E_{1}\end{array}$ & $\begin{array}{l}E_{g} \\
E_{1}\end{array}$ & $\begin{array}{l}A_{2 g} \\
A_{2}\end{array}$ & $\begin{array}{l}A_{1 g} \\
A_{1}\end{array}$ & $\begin{array}{l}E_{g} \\
E_{1}\end{array}$ & $\begin{array}{l}E_{g} \\
E_{1}\end{array}$ & $\begin{array}{l}B_{1 \mathrm{~g}} \\
E_{2}\end{array}$ & $\begin{array}{l}B_{2 g} \\
E_{2}\end{array}$ \\
\hline $\begin{array}{l}\mathrm{D}_{3 \hbar} \\
\mathrm{D}_{3 d}\end{array}$ & $\begin{array}{l}E^{\prime} \\
E_{u}\end{array}$ & $\begin{array}{l}E^{\prime} \\
E_{u}\end{array}$ & $\begin{array}{l}A_{2}^{\prime \prime} \\
A_{2 u}\end{array}$ & $\begin{array}{l}E^{\prime \prime} \\
E_{g}\end{array}$ & $\begin{array}{l}E^{\prime \prime} \\
E_{g}\end{array}$ & $\begin{array}{l}A_{2}^{\prime} \\
A_{2 g}\end{array}$ & $\begin{array}{l}A_{1}^{\prime} \\
A_{1 g}\end{array}$ & $\begin{array}{l}E^{\prime \prime} \\
E_{g}\end{array}$ & $\begin{array}{l}E^{\prime \prime} \\
E_{g}\end{array}$ & $\begin{array}{l}E^{\prime} \\
E_{g}\end{array}$ & $\begin{array}{l}E^{\prime} \\
E_{g}\end{array}$ \\
\hline $\begin{array}{l}\mathrm{D}_{2 d} \\
\mathrm{C}_{40}\end{array}$ & $\begin{array}{l}E \\
E\end{array}$ & $\begin{array}{l}E \\
E\end{array}$ & $\begin{array}{l}B_{2} \\
A_{1}\end{array}$ & $\begin{array}{l}E \\
E\end{array}$ & $\begin{array}{l}E \\
E\end{array}$ & $\begin{array}{l}A_{2} \\
A_{2}\end{array}$ & $\begin{array}{l}A_{1} \\
A_{1}\end{array}$ & $\begin{array}{l}E \\
E\end{array}$ & $\begin{array}{l}E \\
E\end{array}$ & $\begin{array}{l}B_{1} \\
B_{1}\end{array}$ & $\begin{array}{l}B_{2} \\
B_{2}\end{array}$ \\
\hline $\begin{array}{l}\mathrm{D}_{2 \hbar} \\
\mathrm{C}_{3 v}\end{array}$ & $\begin{array}{l}B_{2 u} \\
E\end{array}$ & $\begin{array}{l}B_{1 u} \\
E\end{array}$ & $\begin{array}{l}A_{2 u} \\
A_{1}\end{array}$ & $\begin{array}{l}B_{2 g} \\
E\end{array}$ & $\begin{array}{l}B_{1 g} \\
E\end{array}$ & $\begin{array}{l}A_{2 g} \\
A_{2}\end{array}$ & $\begin{array}{l}A_{1 g} \\
A_{1}\end{array}$ & $\begin{array}{l}B_{1 g} \\
E\end{array}$ & $\begin{array}{l}B_{2 g} \\
E\end{array}$ & ${ }_{E}^{A_{1 g}}$ & $\frac{A_{2 g}}{E}$ \\
\hline $\begin{array}{l}\mathrm{C}_{20} \\
\mathrm{C}_{2 h}\end{array}$ & $\begin{array}{l}B_{1} \\
B_{u}\end{array}$ & $\begin{array}{l}B_{2} \\
B_{u}\end{array}$ & $\begin{array}{l}A_{1} \\
A_{u}\end{array}$ & $\begin{array}{l}B_{2} \\
B_{g}\end{array}$ & $\begin{array}{l}B_{1} \\
B_{g}\end{array}$ & $\begin{array}{l}A_{2} \\
A_{g}\end{array}$ & $\begin{array}{l}A_{1} \\
A_{g}\end{array}$ & $\begin{array}{l}B_{1} \\
B_{g}\end{array}$ & $\begin{array}{l}B_{2} \\
B_{g}\end{array}$ & $\begin{array}{l}A_{1} \\
A_{g}\end{array}$ & $\begin{array}{l}A_{2} \\
A_{g}\end{array}$ \\
\hline \multirow[t]{2}{*}{$\mathrm{C}_{s}$} & $A^{\prime}$ & $A^{\prime}$ & $A^{\prime \prime}$ & $A^{\prime \prime}$ & $A^{\prime \prime}$ & $A^{\prime}$ & $A^{\prime}$ & $A^{\prime \prime}$ & $A^{\prime \prime}$ & $A^{\prime}$ & $A^{\prime}$ \\
\hline & $x+i y$ & $x-i y$ & $z$ & $L_{x}+i L_{y}$ & $L_{x}-i L_{y}$ & $L_{z}$ & $Z^{2}$ & $(x+i y) z$ & $(x-i y) z$ & $(x+i y)^{2}$ & $(x-i y)^{2}$ \\
\hline $\mathrm{C}_{\infty h}$ & $C_{1 u}$ & $C_{-1 u}$ & $A_{u}$ & $C_{1 g}$ & $C_{-1 g}$ & $A_{g}$ & $A_{g}$ & $C_{1 g}$ & $C_{-1 g}$ & $C_{2 g}$ & $C_{-2 g}$ \\
\hline $\begin{array}{l}\mathrm{C}_{6 h} \\
\mathrm{C}_{4 h}\end{array}$ & $\begin{array}{l}C_{1 u} \\
C_{1 u}\end{array}$ & $\begin{array}{l}C_{t u} \\
C_{3 u}\end{array}$ & $\begin{array}{l}A_{u} \\
A_{u}\end{array}$ & $\begin{array}{l}C_{1 g} \\
C_{1 g}\end{array}$ & $\begin{array}{l}C_{5 g} \\
C_{3 g}\end{array}$ & $\begin{array}{l}A_{g} \\
A_{g}\end{array}$ & $\begin{array}{l}A_{g} \\
A_{g}\end{array}$ & $\begin{array}{l}C_{1 g} \\
C_{1 g}\end{array}$ & $\begin{array}{l}C_{5 g} \\
C_{3 g}\end{array}$ & $\begin{array}{l}C_{2 g} \\
B_{g}\end{array}$ & $\begin{array}{l}C_{4 g} \\
B_{g}\end{array}$ \\
\hline $\begin{array}{l}\mathrm{C}_{3 h} \\
\mathrm{~S}_{6}\end{array}$ & $\begin{array}{l}C_{1}^{\prime} \\
C_{1 u}\end{array}$ & $\begin{array}{l}C_{2}^{\prime} \\
C_{2 u}\end{array}$ & $\begin{array}{l}A^{\prime \prime} \\
A_{u}\end{array}$ & $\begin{array}{l}C_{1}^{\prime \prime} \\
C_{18}^{\prime}\end{array}$ & $\begin{array}{l}C_{2}^{\prime} \\
C_{28}\end{array}$ & $\begin{array}{l}A^{\prime} \\
A_{g}\end{array}$ & $\begin{array}{l}A^{\prime} \\
A_{g}\end{array}$ & $\begin{array}{l}C_{1}^{\prime \prime} \\
C_{1 g}\end{array}$ & $\begin{array}{l}C_{2}^{\prime \prime} \\
C_{2 g}^{\prime}\end{array}$ & $\begin{array}{l}C_{2}^{\prime} \\
C_{2 g}\end{array}$ & $\begin{array}{l}C_{1}^{\prime} \\
C_{1 g}^{\prime}\end{array}$ \\
\hline$S_{4}$ & $C_{1}$ & $C_{3}$ & $B$ & $C_{3}$ & $C_{1}$ & $A$ & $A$ & $C_{3}$ & $C_{1}$ & $B$ & $B$ \\
\hline
\end{tabular}

a See text.

of the quadrupole interaction. For example, the reduction of $\mathscr{2}_{g}$ under $\mathrm{O}_{h}$ yields the following as a basis:

$$
\begin{gathered}
E:\left[-\frac{\sqrt{6}}{3} \mathrm{C}_{2}^{1}+\frac{\sqrt{3}}{3} \mathrm{C}_{2}^{2}\right] ;\left[-\frac{\sqrt{6}}{3} \mathrm{~S}_{2}^{1}+\frac{\sqrt{3}}{3} \mathrm{~S}_{2}^{2}\right] \\
T_{2}:\left[\frac{\sqrt{3}}{3} \mathrm{C}_{2}^{1}+\frac{\sqrt{6}}{3} \mathrm{C}_{2}^{2}\right] ;\left[\frac{\sqrt{3}}{3} \mathrm{~S}_{2}^{1}+\frac{\sqrt{6}}{3} \mathrm{~S}_{2}^{2}\right] ;\left[\mathrm{C}_{2}^{0}\right]
\end{gathered}
$$

While this, of course, yields the same polarization results in the physical sense, the trigonal aspect will not be considered further due to its formal mathematical complexities.

Because of the different transformation properties of these components, they will most generally permit transitions between different states of the perturbed ion. The nonvanishing matrix elements may be deduced by the procedures outlined above (section 4.3). These are the selection rules which are applicable in a case of definite symmetry. Furthermore, the relative amount of any particular component of the multipole interaction present will depend upon the vector potential, and in particular, upon the direction of propagation and state of polarization of the incident radiation. A detailed examination of this latter feature leads to the polarization rules for absorption of radiation.

The selection rules for most of the groups in which we shall be interested (omitting only the very simplest) are given in table 13 . For each rep in the left column, the states to which a particular component of the multipole interaction will permit transitions is shown in the column under that multipole component. Generally, the table parallels table 8 , in that closely related groups are shown with but minor modifications on the same table. Results for pure rotation may be obtained by dropping the " $g$ " - " $u$ " distinction in the tables. In a few cases, the changes in notation and convention are sufficiently farreaching to require a separate table. It is particularly interesting to note the table for $\mathrm{C}_{\infty h}$, appropriate to a uniform magnetic field. If we denote the general rep by $C_{M p}\left(C_{0}=A\right)$, we see that electric dipole transitions have $\Delta M=0$, 
TABLE 13. Selection rules

\begin{tabular}{|c|c|c|c|c|c|c|}
\hline $\mathrm{D}_{\infty h}$ & $z$ & $x, y$ & $L_{z}$ & $L_{x}, L_{y}, x \boldsymbol{z}, y \boldsymbol{z}$ & $Z^{2}$ & $\rho^{2}, x y$ \\
\hline $\begin{array}{l}A_{1 g} \\
A_{1 z}\end{array}$ & $\begin{array}{l}A_{2 s} \\
A_{2 g}\end{array}$ & $\begin{array}{l}E_{1 u} \\
E_{1 g}\end{array}$ & $\begin{array}{l}A_{2 g} \\
A_{2 u}\end{array}$ & $\begin{array}{l}E_{1 g} \\
E_{1 u}\end{array}$ & $\begin{array}{l}A_{1 g} \\
A_{1 u}\end{array}$ & $\begin{array}{l}E_{2 g} \\
E_{2 u}\end{array}$ \\
\hline $\begin{array}{l}A_{2 g} \\
A_{2 u}\end{array}$ & $\begin{array}{l}A_{1 u} \\
A_{1 \mathrm{z}}\end{array}$ & $\begin{array}{l}E_{1 u} \\
E_{1 g}\end{array}$ & $\begin{array}{l}A_{18} \\
A_{14}\end{array}$ & $\begin{array}{l}E_{1 g} \\
E_{1 u}\end{array}$ & $\begin{array}{l}A_{2 g} \\
A_{2 u}\end{array}$ & $\begin{array}{l}E_{2 g} \\
E_{2 u}\end{array}$ \\
\hline $\begin{array}{l}E_{1 g} \\
E_{1 u}\end{array}$ & $\begin{array}{l}E_{1 u} \\
E_{1 g}\end{array}$ & $\begin{array}{l}E_{2 u} A_{1 u} A_{2 u} \\
E_{2 g} A_{1 g} A_{2 g}\end{array}$ & $\begin{array}{l}E_{1 z} \\
E_{1 u}\end{array}$ & $\begin{array}{l}E_{2 g} A_{1 g} A_{2 g} \\
E_{2 u} A_{1 u} A_{2 u}\end{array}$ & $\begin{array}{l}E_{18} \\
E_{1 u}\end{array}$ & $\begin{array}{l}E_{18} E_{38} \\
E_{1 u} E_{3 u}\end{array}$ \\
\hline $\begin{array}{l}E_{2 g} \\
E_{2 \text { tg }}\end{array}$ & $\begin{array}{l}E_{22} \\
E_{23}\end{array}$ & $\begin{array}{l}E_{3 u} E_{1 u} \\
E_{3 g} E_{1 g}\end{array}$ & $\begin{array}{l}E_{2 g} \\
E_{2 u}\end{array}$ & $\begin{array}{l}E_{3 g} E_{1 g} \\
E_{3 u} E_{1 u}\end{array}$ & $\begin{array}{l}E_{2 g} \\
E_{2 u}\end{array}$ & $\begin{array}{l}E_{4 g} A_{1 g} A_{2 g} \\
E_{4 u} A_{1 u} A_{2 u}\end{array}$ \\
\hline $\begin{array}{l}E_{3 g} \\
E_{3 u}\end{array}$ & $\begin{array}{l}E_{3 u} \\
E_{3 g}\end{array}$ & $\begin{array}{l}E_{4 u} E_{2 u} \\
E_{4 g} E_{2 g}^{2}\end{array}$ & $\begin{array}{l}E_{3 g} \\
E_{3 u}\end{array}$ & $\begin{array}{l}E_{4 g} E_{2 g} \\
E_{4 u} E_{2 u}^{2}\end{array}$ & $\begin{array}{l}E_{3 g} \\
E_{3 u}\end{array}$ & $\begin{array}{l}E_{5 g} E_{1 g} \\
E_{5 u} E_{1 u}\end{array}$ \\
\hline
\end{tabular}

(Pattern repeats for higher states)

\begin{tabular}{|c|c|c|c|c|c|c|}
\hline $\begin{array}{l}{ }_{2} S_{18} \\
{ }_{2} S_{1 u}\end{array}$ & $\begin{array}{l}{ }_{2}^{2} S_{1 u} \\
{ }_{2} S_{1 g}\end{array}$ & $\begin{array}{l}{ }_{2} S_{1 u}{ }_{2} S_{3 u s} \\
{ }_{2} S_{1 g} S_{2 g} S_{3 g}\end{array}$ & $\begin{array}{l}{ }_{2} S_{1 g} S_{2} \\
{ }_{2} S_{1 u}\end{array}$ & $\begin{array}{l}{ }_{2} S_{1 g}{ }_{2} S_{3 g} \\
{ }_{2} S_{1 u} S_{2} S_{3 u}\end{array}$ & $\begin{array}{l}{ }_{2} S_{1 g} \\
{ }_{2} S_{1 u}\end{array}$ & $\begin{array}{ll}{ }_{2} S_{3 g} & { }_{2} S_{5 g} \\
{ }_{2} S_{3 u} & { }_{2} S_{5 u}\end{array}$ \\
\hline $\begin{array}{l}{ }_{2} S_{3 z} \\
{ }_{2} S_{3 u}\end{array}$ & $\begin{array}{l}{ }_{2} S_{3 u} \\
{ }_{2} S_{3 g}\end{array}$ & $\begin{array}{ll}{ }_{2}^{2} S_{1 u} & { }_{2} S_{5 u} \\
{ }_{2} S_{1 g} & S_{2} S_{5 g}\end{array}$ & $\begin{array}{l}{ }_{2} S_{3 g} \\
{ }_{2} S_{3 u}\end{array}$ & $\begin{array}{ll}{ }_{2}^{2} S_{1 g} & { }_{2} S_{5 g} \\
{ }_{2} S_{1 u} & { }_{2} S_{5 u}\end{array}$ & $\begin{array}{l}{ }_{2} S_{3 g} \\
{ }_{2} S_{3 u}\end{array}$ & $\begin{array}{ll}{ }_{2} S_{1 g} S_{2} S_{7 g} \\
{ }_{2} S_{1 u} S_{2} S_{7 u}\end{array}$ \\
\hline $\begin{array}{l}{ }_{2} S_{5 g} \\
{ }_{2} S_{5 u}\end{array}$ & $\begin{array}{l}{ }_{2} S_{5 u} \\
{ }_{2} S_{\bar{s}_{z}}\end{array}$ & $\begin{array}{ll}{ }_{2} S_{3 u} & { }_{2} S_{7 u} \\
{ }_{2} S_{3 g} & { }_{2} S_{7 g}\end{array}$ & $\begin{array}{l}{ }_{2} S_{5 g} \\
{ }_{2} S_{5 u}\end{array}$ & $\begin{array}{ll}{ }_{2} S_{3 g} & { }_{2} S_{7 g} \\
{ }_{2} S_{3 u} & { }_{2} S_{7 u}\end{array}$ & $\begin{array}{l}2 S_{5 g} \\
2 S_{5 u}\end{array}$ & $\begin{array}{ll}{ }_{2} S_{1 g} & { }_{2} S_{9 g} \\
{ }_{2} S_{1 u} & { }_{2} S_{9 u}\end{array}$ \\
\hline
\end{tabular}

(Pattern repeats for higher states)

For $C_{\infty v}$, delete " $g$ " and " $u$ " and read the upper left corner as:

\begin{tabular}{c|c}
$C_{\infty \text { v }}$ & $\boldsymbol{z}$ \\
\hline$A_{1}$ & $A_{1}$ \\
$A_{2}$ & $A_{2}$
\end{tabular}

\begin{tabular}{|c|c|c|c|c|c|c|}
\hline $\mathrm{D}_{6 h}$ & $z$ & $x, y$ & $L_{z}$ & $L_{x}, L_{y}, x \boldsymbol{z}, y \boldsymbol{z}$ & $Z^{2}$ & $\rho^{2}, x y$ \\
\hline $\begin{array}{l}A_{1 z} \\
A_{1 u}\end{array}$ & $\begin{array}{l}A_{2 u} \\
A_{2 g}\end{array}$ & $\begin{array}{l}E_{1 u} \\
E_{1 g}\end{array}$ & $\begin{array}{l}A_{2 g} \\
A_{2 u}\end{array}$ & $\begin{array}{l}E_{1 g} \\
E_{1 u}\end{array}$ & $\begin{array}{l}A_{1 g} \\
A_{1 u}\end{array}$ & $\begin{array}{l}E_{2 g} \\
E_{2 u}\end{array}$ \\
\hline $\begin{array}{l}A_{2 g} \\
A_{2 z}\end{array}$ & $\begin{array}{l}A_{1 u} \\
A_{1 g}\end{array}$ & $\begin{array}{l}E_{1 u} \\
E_{1 g}\end{array}$ & $\begin{array}{l}A_{1 g} \\
A_{1 u}\end{array}$ & $\begin{array}{l}E_{18} \\
E_{1 u}\end{array}$ & $\begin{array}{l}A_{2 g} \\
A_{2 u}\end{array}$ & $\begin{array}{l}E_{2 g} \\
E_{2 u}\end{array}$ \\
\hline $\begin{array}{l}B_{18} \\
B_{1 z}\end{array}$ & $\begin{array}{l}B_{2 u} \\
B_{2 g}\end{array}$ & $\begin{array}{l}E_{2 u} \\
E_{2 g}\end{array}$ & $\begin{array}{l}B_{2 g} \\
B_{2 u}\end{array}$ & $\begin{array}{l}E_{2 g} \\
E_{2 u}\end{array}$ & $\begin{array}{l}B_{1 g} \\
B_{1 u}\end{array}$ & $\begin{array}{l}E_{1 g} \\
E_{1 u}\end{array}$ \\
\hline $\begin{array}{l}B_{2 g} \\
B_{2 u}\end{array}$ & $\begin{array}{l}B_{1 u} \\
B_{1 g}\end{array}$ & $\begin{array}{l}E_{2 u} \\
E_{2 z}\end{array}$ & $\begin{array}{l}B_{18} \\
B_{1 u}\end{array}$ & $\begin{array}{l}E_{2 g} \\
E_{2 u}\end{array}$ & $\begin{array}{l}B_{2 g} \\
B_{2 u}\end{array}$ & $\begin{array}{l}E_{1 g} \\
E_{1 u}\end{array}$ \\
\hline $\begin{array}{l}E_{1 g} \\
E_{1 u}\end{array}$ & $\begin{array}{l}E_{1 u} \\
E_{1 g}\end{array}$ & $\begin{array}{l}A_{1 u} A_{2 u} E_{2 u} \\
A_{1 g} A_{2 g} E_{2 g}\end{array}$ & $\begin{array}{l}E_{1 g} \\
E_{1 u}\end{array}$ & $\begin{array}{l}A_{1 g} A_{2 g} E_{2 g} \\
A_{1 u} A_{2 u} E_{2 u}\end{array}$ & $\begin{array}{l}E_{1 g} \\
E_{1 u}\end{array}$ & $\begin{array}{l}B_{1 g} B_{2 g} E_{1 g} \\
B_{1 u} B_{2 u} E_{1 u}\end{array}$ \\
\hline $\begin{array}{l}E_{2 g} \\
E_{2 g}\end{array}$ & $\begin{array}{l}E_{2 u} \\
E_{2 g}\end{array}$ & $\begin{array}{l}B_{1 u} B_{2 u} E_{1 u} \\
B_{1 g} B_{2 g} E_{1 g}\end{array}$ & $\begin{array}{l}E_{2 g} \\
E_{2 u}\end{array}$ & $\begin{array}{l}B_{1 g} B_{2 g} E_{1 g} \\
B_{1 u} B_{2 u} E_{1 u}\end{array}$ & $\begin{array}{l}E_{2 g} \\
E_{2 u}\end{array}$ & $\begin{array}{l}A_{1 g} A_{2 g} E_{2 g} \\
A_{1 u} A_{2 u} E_{2 u}\end{array}$ \\
\hline $\begin{array}{l}D_{1 / 2 g} \\
D_{1 / 24}\end{array}$ & $\begin{array}{l}D_{1 / 24} \\
D_{1 / 28}\end{array}$ & $\begin{array}{l}{ }_{2} S_{1 u} D_{1 / 2 u} \\
{ }_{2} S_{1 g} D_{1 / 2 g}\end{array}$ & $\begin{array}{l}D_{1 / 2 g} \\
D_{1 / 2 u}\end{array}$ & $\begin{array}{l}{ }_{2} S_{1 g} D_{1 / 2 g} \\
{ }_{2} S_{1 u} D_{1 / 2 u}\end{array}$ & $\begin{array}{l}D_{1 / 2 g} \\
D_{1 / 2 u}\end{array}$ & $\begin{array}{ll}{ }_{2} S_{1 g} S_{2} S_{2 g} \\
{ }_{2} S_{1 u}{ }_{2} S_{2 u}\end{array}$ \\
\hline $\begin{array}{l}{ }_{2} S_{1 g} \\
{ }_{2} S_{1 u}\end{array}$ & ${ }_{2}^{2} S_{1 u}$ & $\begin{array}{l}{ }_{2} S_{2 u} D_{1 / 2 u} \\
{ }_{2} S_{2 g} D_{1 / 2 g}\end{array}$ & $\begin{array}{l}{ }_{2} S_{1 g} \\
{ }_{2} S_{1 u}\end{array}$ & $\begin{array}{l}{ }_{2} S_{2 g} D_{1 / 2 g} \\
{ }_{2} S_{2 u} D_{1 / 2 u}\end{array}$ & $\begin{array}{l}{ }_{2} S_{1 g} \\
{ }_{2} S_{1 u}\end{array}$ & $\begin{array}{l}{ }_{2} S_{2 g} D_{1 / 2 g} \\
{ }_{2} S_{2 u} D_{1 / 2 u}\end{array}$ \\
\hline $\begin{array}{l}{ }_{2} S_{2 g} \\
{ }_{2} S_{2 u}\end{array}$ & ${ }_{2}^{2} S_{2 u}$ & $\begin{array}{l}{ }_{2} S_{1 u 2} S_{2 u} \\
{ }_{2} S_{1 g} S_{2 g} S_{2 g}\end{array}$ & ${ }_{2}^{2} S_{2 g} S_{2 u}$ & $\begin{array}{ll}{ }_{2} S_{1 g}{ }_{2} S_{2 g} \\
{ }_{2} S_{1 u} & 2 S_{2 u}\end{array}$ & $\begin{array}{l}{ }_{2} S_{2 g} \\
{ }_{2} S_{2 u}\end{array}$ & $\begin{array}{l}{ }_{2} S_{1 g} D_{1 / 2 g} \\
{ }_{2} S_{1 u} D_{1 / 2 u}\end{array}$ \\
\hline
\end{tabular}

For $C_{6 v}$, drop " $g$ "- " $u$ " and amend upper left corner to read:

\begin{tabular}{c|c}
$C_{60}$ & $z$ \\
\hline & \\
\hline$A_{1}$ & $A_{1}$ \\
$A_{2}$ & $A_{2}$ \\
$B_{1}$ & $B_{1}$ \\
$B_{2}$ & $B_{2}$
\end{tabular}


TABLE 13. Selection rules-Continued

\begin{tabular}{|c|c|c|c|c|c|c|c|}
\hline $\mathrm{D}_{\sharp h}$ & $z$ & $x, y$ & $L_{z}$ & $L_{x}, L_{y}, x \boldsymbol{z}, y \boldsymbol{z}$ & $Z^{2}$ & $\rho^{2}$ & $x y$ \\
\hline $\begin{array}{l}A_{1 g} \\
A_{1 u}\end{array}$ & $\begin{array}{l}A_{2 u} \\
A_{2 g}\end{array}$ & $\begin{array}{l}E_{u} \\
E_{\mathrm{g}}\end{array}$ & $\begin{array}{l}A_{2 g} \\
A_{2 u}\end{array}$ & $\begin{array}{l}E_{g} \\
E_{u}\end{array}$ & $\begin{array}{l}A_{1 g} \\
A_{1 u}\end{array}$ & $\begin{array}{l}B_{1 g} \\
B_{1 u}\end{array}$ & $\begin{array}{l}B_{2 g} \\
B_{2 u}\end{array}$ \\
\hline $\begin{array}{l}A_{2 g} \\
A_{2 u}\end{array}$ & $\begin{array}{l}A_{1 u} \\
A_{1 g}\end{array}$ & $\begin{array}{l}E_{u} \\
E_{g}\end{array}$ & $\begin{array}{l}A_{18} \\
A_{1 u}\end{array}$ & $\begin{array}{l}E_{g} \\
E_{u}\end{array}$ & $\begin{array}{l}A_{2 g} \\
A_{2 u}\end{array}$ & $\begin{array}{l}B_{2 g} \\
B_{2 u}\end{array}$ & $\begin{array}{l}B_{1 g} \\
B_{1 u}\end{array}$ \\
\hline $\begin{array}{l}E_{1 g} \\
B_{1 u}\end{array}$ & $\begin{array}{l}B_{2 u} \\
B_{2 g}\end{array}$ & $\begin{array}{l}E_{u} \\
E_{g}\end{array}$ & $\begin{array}{l}B_{2 g} \\
B_{2 u}\end{array}$ & $\begin{array}{l}E_{g} \\
E_{u}\end{array}$ & $\begin{array}{l}B_{1 g} \\
B_{1 u}\end{array}$ & $\begin{array}{l}A_{1 \varepsilon} \\
A_{1 u}\end{array}$ & $\begin{array}{l}A_{2 g} \\
A_{2 u}\end{array}$ \\
\hline $\begin{array}{l}B_{2 g} \\
B_{2 u}\end{array}$ & $\begin{array}{l}B_{1 u} \\
B_{1 g}\end{array}$ & $\begin{array}{l}E_{u} \\
E_{g}\end{array}$ & $\begin{array}{l}B_{1 g} \\
B_{1 u}\end{array}$ & $\begin{array}{l}E_{g} \\
E_{u}\end{array}$ & $\begin{array}{l}B_{2 g} \\
B_{2 u}\end{array}$ & $\begin{array}{l}A_{2 g} \\
A_{2 u}\end{array}$ & $\begin{array}{l}A_{1 g} \\
A_{1 u}\end{array}$ \\
\hline $\begin{array}{l}E_{g} \\
E_{u}\end{array}$ & $\begin{array}{l}E_{u} \\
E_{g}\end{array}$ & $\begin{array}{l}A_{1 u} A_{2 u} B_{1 u} B_{2 u} \\
A_{1 g} A_{2 g} B_{1 g} B_{2 g}\end{array}$ & $\begin{array}{l}E_{g} \\
E_{u}\end{array}$ & $\begin{array}{l}A_{1 g} A_{2 g} B_{18} B_{2 g} \\
A_{1 u} A_{2 u} B_{1 u} B_{2 u}\end{array}$ & $\begin{array}{l}E_{g} \\
E_{u}\end{array}$ & $\begin{array}{l}E_{g} \\
E_{u}\end{array}$ & $\begin{array}{l}E_{g} \\
E_{u}\end{array}$ \\
\hline $\begin{array}{l}D_{1 / 28} \\
D_{3 / 2 u}\end{array}$ & $\begin{array}{l}D_{1 / 2 u} \\
D_{1 / 28}\end{array}$ & $\begin{array}{l}{ }_{2} S_{u} D_{1 / 2 u} \\
{ }_{2} S_{g} D_{1 / 28}\end{array}$ & $\begin{array}{l}D_{1 / 2 g} \\
D_{1 / 2 u}\end{array}$ & $\begin{array}{l}{ }_{2} S_{g} D_{3 / 28} \\
{ }_{2} S_{u} D_{3 / 2 u}\end{array}$ & $\begin{array}{l}D_{1 / 28} \\
D_{1 / 2 u}\end{array}$ & ${ }_{2}^{2} S_{g} S_{u}$ & ${ }_{2}^{2} S_{\varepsilon}$ \\
\hline${ }_{2}^{2} S_{g}$ & ${ }_{2}^{2} S_{u}$ & $\begin{array}{l}{ }_{2} S_{u} D_{1 / 2 u} \\
{ }_{2} S_{8} D_{3 / 28}\end{array}$ & ${ }_{2} S_{{ }_{u}} S_{u}$ & $\begin{array}{l}{ }_{2} S_{\varepsilon} D_{3 / 2 g} \\
{ }_{2} S_{u} D_{3 / 2 u}\end{array}$ & ${ }_{2}^{2} S_{s}$ & $\begin{array}{l}D_{1 / 2 g} \\
D_{1 / 2 u}\end{array}$ & $\begin{array}{l}D_{3 / 28} \\
D_{3 / 2 u}\end{array}$ \\
\hline
\end{tabular}

For $\mathrm{C}_{4_{v}}$ : Drop " $g$ " and " $u$ " and read $z$ in $Z^{2}$ column.

For $\mathrm{D}_{2 d}$ : Drop " $g$ " and " $u$ " and read $z$ in $x y$ column.

\begin{tabular}{|c|c|c|c|c|c|}
\hline $\mathrm{D}_{3 h}$ & $z$ & $x, y, \rho^{2}, x y$ & $L_{z}$ & $L_{x}, L_{y}, x z, y z$ & $Z^{2}$ \\
\hline $\begin{array}{l}A_{1}^{\prime} \\
A_{2}^{\prime}\end{array}$ & $\begin{array}{l}A_{2, \prime}^{\prime \prime} \\
A_{1}^{\prime \prime}\end{array}$ & $\begin{array}{l}E^{\prime} \\
E^{\prime}\end{array}$ & $\begin{array}{l}A_{2}^{\prime} \\
A_{1}^{\prime}\end{array}$ & $\begin{array}{l}E^{\prime \prime} \\
E^{\prime \prime}\end{array}$ & $\begin{array}{l}A_{1}^{\prime} \\
A_{2}^{\prime}\end{array}$ \\
\hline $\begin{array}{l}A_{1}^{\prime \prime} \\
A_{2}^{\prime \prime}\end{array}$ & $\begin{array}{l}A_{2}^{\prime} \\
A_{1}^{\prime}\end{array}$ & $\begin{array}{l}E^{\prime \prime} \\
E^{\prime \prime}\end{array}$ & $\begin{array}{l}A_{2}^{\prime \prime} \\
A_{1}^{\prime \prime}\end{array}$ & $\begin{array}{l}E^{\prime} \\
E^{\prime}\end{array}$ & $\begin{array}{l}A_{1,}^{\prime \prime} \\
A_{2}^{\prime \prime}\end{array}$ \\
\hline $\begin{array}{l}E^{\prime} \\
E^{\prime \prime}\end{array}$ & $\begin{array}{l}E^{\prime \prime} \\
E^{\prime}\end{array}$ & $\begin{array}{l}A_{1}^{\prime} A_{2}^{\prime} E^{\prime} \\
A_{1}^{\prime \prime} A_{2}^{\prime \prime} E^{\prime \prime}\end{array}$ & $\begin{array}{l}E^{\prime} \\
E^{\prime \prime}\end{array}$ & $\begin{array}{l}A_{1}^{\prime \prime} A_{2}^{\prime \prime} E^{\prime \prime} \\
A_{1}^{\prime} A_{2}^{\prime} E^{\prime}\end{array}$ & $\begin{array}{l}E^{\prime} \\
E^{\prime \prime}\end{array}$ \\
\hline $\begin{array}{l}D_{3 / 2} \\
2 S_{1} \\
{ }_{2} S_{2}\end{array}$ & $\begin{array}{l}{ }_{2} S_{2} \\
{ }_{2} S_{1} \\
D_{1 / 2}\end{array}$ & $\begin{array}{l}2 S_{1} S_{2} S_{2} \\
D_{1 / 2} \\
D_{1 / 2}{ }_{2} S_{2} \\
D_{1 / 2} S_{1}\end{array}$ & $\begin{array}{l}D_{1 / 2} \\
{ }_{2} S_{1} \\
{ }_{2} S_{2}\end{array}$ & $\begin{array}{l}D_{3 / 2}{ }_{2} S_{1} \\
{ }_{2} S_{2} D_{3 / 3} \\
{ }_{2} S_{1} \\
{ }_{2} S_{2} S_{2}\end{array}$ & $\begin{array}{l}D_{3 / 2} \\
{ }_{2} S_{1} \\
{ }_{2} S_{2}\end{array}$ \\
\hline
\end{tabular}

\pm 1 , with change in parity, magnetic dipole transitions have $\Delta M=0, \pm 1$, with no change in parity, while electric quadrupole transitions have $\Delta M=0, \pm 1, \pm 2$, with no change in parity.

In order to examine in detail the polarization effects, let us consider a set of orthogonal unit vectors in spherical coordinates. $\mathbf{r}_{0}=\sin \theta \cos \phi \mathbf{i}+\sin \theta \sin \phi \mathbf{j}+\cos \theta \mathbf{k}$

$\boldsymbol{\theta}_{0}=\frac{\partial \mathbf{r}_{0}}{\partial \theta}=\cos \theta \cos \phi \mathbf{i}+\cos \theta \sin \phi \mathbf{j}-\sin \theta \mathbf{k}$

$\phi_{0}=\frac{1}{\sin \theta} \frac{\partial \mathbf{r}_{0}}{\partial \phi}=-\sin \phi \mathbf{i}+\cos \phi \mathbf{j}$ 
TABLE 13. Selection rules-Continued

\begin{tabular}{|c|c|c|c|c|c|}
\hline $\mathrm{D}_{3 d}$ & $z$ & $x, y$ & $L_{z}$ & $\begin{array}{l}\rho^{2}, x y \\
L_{x}, L_{y} \\
x z, y z\end{array}$ & $Z^{2}$ \\
\hline $\begin{array}{l}A_{1 g} \\
A_{1 u}\end{array}$ & $\begin{array}{l}A_{2 u} \\
A_{2 g}\end{array}$ & $\begin{array}{l}E_{u} \\
E_{g}\end{array}$ & $\begin{array}{l}A_{2 g} \\
A_{2 u}\end{array}$ & $\begin{array}{l}E_{g} \\
E_{u}\end{array}$ & $\begin{array}{l}A_{1 g} \\
A_{1 u}\end{array}$ \\
\hline $\begin{array}{l}A_{2 g} \\
A_{2 u}\end{array}$ & $\begin{array}{l}A_{1 u} \\
A_{1 g}\end{array}$ & $\begin{array}{l}E_{u} \\
E_{g}\end{array}$ & $\begin{array}{l}A_{1 g} \\
A_{1 u}\end{array}$ & $\underset{E_{g}}{E_{u}}$ & $\begin{array}{l}A_{2 g} \\
A_{2 u}\end{array}$ \\
\hline $\begin{array}{l}E_{g} \\
E_{u}\end{array}$ & $\begin{array}{l}E_{u} \\
E_{g}\end{array}$ & $\begin{array}{lll}A_{1 u} & A_{2 u} & E_{u} \\
A_{1 g} & A_{2 g} & E_{g}\end{array}$ & $\begin{array}{l}E_{g} \\
E_{u}\end{array}$ & $\begin{array}{l}A_{1 g} A_{2 g} \\
A_{1 u} \\
A_{1 u} \\
A_{2 u} \\
E_{u}\end{array}$ & $\begin{array}{l}E_{g} \\
E_{u}\end{array}$ \\
\hline $\begin{array}{l}S_{1 g} \\
S_{1 u}\end{array}$ & $\begin{array}{l}S_{3 u} \\
S_{3 g}\end{array}$ & $\begin{array}{l}D_{1 / 2 u} \\
D_{1 / 2 g}\end{array}$ & $\begin{array}{l}S_{3 g} \\
S_{3 u}\end{array}$ & $\begin{array}{l}D_{1 / 2 \mathrm{~g}} \\
D_{3 / 2 u}\end{array}$ & $\begin{array}{l}S_{1 g} \\
S_{1 u}\end{array}$ \\
\hline $\begin{array}{l}S_{3 g} \\
S_{3 g}\end{array}$ & $\begin{array}{l}S_{1 u} \\
S_{1 \mathrm{~g}}\end{array}$ & $\begin{array}{l}D_{1 / 2 u} \\
D_{1 / 2 g}\end{array}$ & $\begin{array}{l}S_{1 g} \\
S_{1 u}\end{array}$ & $\begin{array}{l}D_{1 / 2 g} \\
D_{1 / 2 u}\end{array}$ & $\begin{array}{l}S_{3 g} \\
S_{3 u}\end{array}$ \\
\hline $\begin{array}{l}D_{1 / 28} \\
D_{1 / 2 u}\end{array}$ & $\begin{array}{l}D_{1 / 2 u} \\
D_{1 / 28}\end{array}$ & $\begin{array}{ll}S_{1 u} & S_{3 u} \\
S_{1 g} & D_{1 / 1 u} \\
S_{3 g} & D_{3 / 4 g}\end{array}$ & $\begin{array}{l}D_{3 / 2 g} \\
D_{3 / 2 u}\end{array}$ & 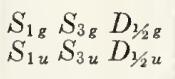 & $\begin{array}{l}D_{3 / 2 g} \\
D_{3 / 2 u}\end{array}$ \\
\hline
\end{tabular}

For $\mathrm{C}_{3}$ : Drop " $g$ " and " $u$ " and read $z$ in $Z^{2}$ column.

\begin{tabular}{|c|c|c|c|c|c|c|c|}
\hline $\mathrm{D}_{2 h}$ & $z$ & $x$ & $y$ & $L_{2}, x y$ & $L_{x}, y z$ & $L_{y}, x z$ & $Z^{2}, \rho^{2}$ \\
\hline $\begin{array}{l}A_{1 g} \\
A_{1 u}\end{array}$ & $\begin{array}{l}A_{2 u} \\
A_{2 g}\end{array}$ & $\begin{array}{l}B_{2 u} \\
B_{2 g}\end{array}$ & $\begin{array}{l}B_{1 u} \\
B_{1 \mathrm{~g}}\end{array}$ & $\begin{array}{l}A_{2 z} \\
A_{2 u}\end{array}$ & $\begin{array}{l}B_{2 g} \\
B_{2 u}\end{array}$ & $\begin{array}{l}B_{1 g} \\
B_{1 u}\end{array}$ & $\begin{array}{l}A_{1 g} \\
A_{1 u}\end{array}$ \\
\hline $\begin{array}{l}A_{2 g} \\
A_{2 u}\end{array}$ & $\begin{array}{l}A_{1 u} \\
A_{1 g}\end{array}$ & $\begin{array}{l}B_{1 u} \\
B_{1 g}\end{array}$ & $\begin{array}{l}B_{2 u} \\
B_{2 g}\end{array}$ & $\begin{array}{l}A_{1 g} \\
A_{1 u}\end{array}$ & $\begin{array}{l}B_{1 g} \\
B_{1 u}\end{array}$ & $\begin{array}{l}B_{2 g} \\
B_{2 u}\end{array}$ & $\begin{array}{l}A_{2 g} \\
A_{2 u}\end{array}$ \\
\hline $\begin{array}{l}B_{1 g} \\
B_{1 u}\end{array}$ & $\begin{array}{l}B_{2 u} \\
B_{2 g}\end{array}$ & $\begin{array}{l}A_{2 u} \\
A_{2 g}\end{array}$ & $\begin{array}{l}A_{1 u} \\
A_{1 g}\end{array}$ & $\begin{array}{l}B_{2 g} \\
B_{2 u}\end{array}$ & $\begin{array}{l}A_{2 g} \\
A_{2 u}\end{array}$ & $\begin{array}{l}A_{1 g} \\
A_{1 u}\end{array}$ & $\begin{array}{l}B_{1 g} \\
B_{1 u}\end{array}$ \\
\hline $\begin{array}{l}B_{2 g} \\
B_{2 u}\end{array}$ & $\begin{array}{l}B_{1 u} \\
B_{1 g}\end{array}$ & $\begin{array}{l}A_{1 u} \\
A_{1 g}\end{array}$ & $\begin{array}{l}A_{2 u} \\
A_{2 z}\end{array}$ & $\begin{array}{l}B_{1 g} \\
B_{1 u}\end{array}$ & $\begin{array}{l}A_{1 g} \\
A_{1 u}\end{array}$ & $\begin{array}{l}A_{2 g} \\
A_{2 u}\end{array}$ & $\begin{array}{l}B_{2 g} \\
B_{2 u}\end{array}$ \\
\hline \multirow[t]{2}{*}{$\begin{array}{l}D_{3 / 28} \\
D_{3 / 2 u}\end{array}$} & $\begin{array}{l}D_{1 / 2 u} \\
D_{1 / 25}\end{array}$ & $\begin{array}{l}D_{3 / 2 u} \\
D_{3 / 2 \mathrm{~g}}\end{array}$ & $\begin{array}{l}D_{1 / 2 u} \\
D_{1 / 2 g}\end{array}$ & $\begin{array}{l}D_{1 / 2 g} \\
D_{3 / 2 u}\end{array}$ & $\begin{array}{l}D_{1 / 2 g} \\
D_{3 / 2 u}\end{array}$ & $\begin{array}{l}D_{1 / 2 g} \\
D_{1 / 2 u}\end{array}$ & $\begin{array}{l}D_{3 / 2 g} \\
D_{1 / 2 u}\end{array}$ \\
\hline & & $\begin{array}{l}y, L_{x} \\
y z\end{array}$ & $\begin{array}{l}x, L_{y} \\
x z\end{array}$ & $L_{z}, x y$ & & & $z, Z^{2}, \rho^{2}$ \\
\hline
\end{tabular}

$\mathrm{C}_{2 v}$ : Omit " $g$ "- " $u$ " and read bottom of eolumn.

\begin{tabular}{l|l|l|l|l|l|l}
\hline \hline \multicolumn{1}{c|}{$S_{6}$} & $x+i y$ & $x-i y$ & $z$ & $\begin{array}{l}(x+i y) z \\
L_{x}+i L_{y} \\
(x-i y)^{2}\end{array}$ & $\begin{array}{l}(x-i y) z \\
L_{x}-i L_{y} \\
(x+i y)^{2}\end{array}$ & $L_{z} Z^{2}$ \\
\hline$A_{g}$ & $C_{1 u}$ & $C_{2 u}$ & $A_{u}$ & $C_{1 g}$ & $C_{2 g}$ & $A_{g}$ \\
$A_{u}$ & $C_{1 g}$ & $C_{2 g}$ & $A_{g}$ & $C_{1 u}$ & $C_{2 u}$ & $A_{u}$ \\
$C_{1 g}$ & $C_{2 u}$ & $A_{u}$ & $C_{1 u}$ & $C_{2 g}$ & $A_{g}$ & $C_{1 g}$ \\
$C_{1 u}$ & $C_{2 g}$ & $A_{g}$ & $C_{1 g}$ & $C_{2 u}$ & $A_{u}$ & $C_{1 u}$ \\
$C_{2 g}$ & $A_{u}$ & $C_{1 u}$ & $C_{2 u}$ & $A_{g}$ & $C_{1 g}$ & $C_{2 g}$ \\
$C_{2 u}$ & $A_{g}$ & $C_{1 g}$ & $C_{2 g}$ & $A_{u}$ & $C_{1 u}$ & $C_{2 u}$ \\
$S_{1 g}$ & $S_{3 u}$ & $S_{5 u}$ & $S_{1 u}$ & $S_{3 g}$ & $S_{5 g}$ & $S_{1 g}$ \\
$S_{1 u}$ & $S_{3 g}$ & $S_{5 g}$ & $S_{1 g}$ & $S_{3 u}$ & $S_{5 u}$ & $S_{1 u}$ \\
$S_{3 g}$ & $S_{5 u}$ & $S_{1 u}$ & $S_{3 u}$ & $S_{5 g}$ & $S_{1 g}$ & $S_{3 g}$ \\
$S_{3 u}$ & $S_{5 g}$ & $S_{1 g}$ & $S_{3 g}$ & $S_{5 u}$ & $S_{1 u}$ & $S_{3 u}$ \\
$S_{5 g}$ & $S_{1 u}$ & $S_{3 u}$ & $S_{5 u}$ & $S_{1 g}$ & $S_{3 g}$ & $S_{5 g}$ \\
$S_{5 u}$ & $S_{1 g}$ & $S_{3 g}$ & $S_{5 g}$ & $S_{1 u}$ & $S_{3 u}$ & $S_{5 u}$ \\
\hline
\end{tabular}


TABLE 13. Selection rules-Continued

\begin{tabular}{|c|c|c|c|c|c|c|c|c|}
\hline $\mathrm{C}_{\infty h h}$ & $x+i y$ & $x-i y$ & $z$ & $\begin{array}{l}L_{x}+i L_{y} \\
(x+i y) z\end{array}$ & $\begin{array}{l}L_{x}-i L_{y} \\
(x-i y) z\end{array}$ & $L_{2}, Z^{2}$ & $(x+i y)^{2}$ & $(x-i y)^{2}$ \\
\hline $\begin{array}{l}A_{g} \\
A_{u}\end{array}$ & $\begin{array}{l}C_{1 u} \\
C_{1 g}\end{array}$ & $\begin{array}{l}C_{-1 u} \\
C_{-1 g}\end{array}$ & $\begin{array}{l}A_{u} \\
A_{g}\end{array}$ & $\begin{array}{l}C_{1 g} \\
C_{1 u}\end{array}$ & $\begin{array}{l}C_{-1 g} \\
C_{-1 u}\end{array}$ & $\begin{array}{l}A_{g} \\
A_{u}\end{array}$ & $\begin{array}{l}C_{2 g} \\
C_{2 u}\end{array}$ & $\begin{array}{l}C_{-2 g} \\
C_{-2 u}\end{array}$ \\
\hline $\begin{array}{l}C_{1 g} \\
C_{1 u} \\
C_{-1 g} \\
C_{-1 u}\end{array}$ & $\begin{array}{l}C_{2 u} \\
C_{2 g} \\
A_{u} \\
A_{g}\end{array}$ & $\begin{array}{l}A_{u} \\
A_{g} \\
C_{-2 u} \\
C_{-2 g}\end{array}$ & $\begin{array}{l}C_{1 u} \\
C_{1 g} \\
C_{-1 u} \\
C_{-1 g}^{-1}\end{array}$ & $\begin{array}{l}C_{2 g} \\
C_{2 u} \\
A_{g} \\
A_{u}\end{array}$ & $\begin{array}{l}A_{g} \\
A_{u} \\
C_{-2 g} \\
C_{-2 u}\end{array}$ & $\begin{array}{l}C_{1 g} \\
C_{1 u} \\
C_{-1 g} \\
C_{-1 u}\end{array}$ & $\begin{array}{l}C_{3 g} \\
C_{3 u} \\
C_{1 g} \\
C_{1 u}\end{array}$ & $\begin{array}{l}C_{-1 g} \\
C_{-1 u} \\
C_{-3 g} \\
C_{-3 u}\end{array}$ \\
\hline $\begin{array}{l}C_{2 g} \\
C_{2 u} \\
C_{-2 g} \\
C_{-2 u}\end{array}$ & $\begin{array}{l}C_{3 u} \\
C_{3 g} \\
C_{-1 u} \\
C_{-1 g}\end{array}$ & $\begin{array}{l}C_{1 u} \\
C_{1 g} \\
C_{-3 u} \\
C_{-3 g}\end{array}$ & $\begin{array}{l}C_{2 u} \\
C_{2 g} \\
C_{-2 u} \\
C_{-2 g}^{-2}\end{array}$ & $\begin{array}{l}C_{3 g} \\
C_{3 u} \\
C_{-1 g} \\
C_{-1 u}\end{array}$ & $\begin{array}{l}C_{1 g} \\
C_{1 u} \\
C_{-3 g} \\
C_{-3 u}\end{array}$ & $\begin{array}{l}C_{2 g} \\
C_{2 u} \\
C_{-2 g} \\
C_{-2 u}\end{array}$ & $\begin{array}{l}C_{4 g} \\
C_{4 u} \\
A_{g} \\
A_{u}\end{array}$ & $\begin{array}{l}A_{g} \\
A_{u} \\
C_{-48} \\
C_{-4 u}\end{array}$ \\
\hline $\begin{array}{l}C_{3 g} \\
C_{3 u} \\
C_{-3 g} \\
C_{-3 u}\end{array}$ & $\begin{array}{l}C_{4 u} \\
C_{4 g} \\
C_{-2, z} \\
C_{-2 g}\end{array}$ & $\begin{array}{l}C_{2 u} \\
C_{? g} \\
C_{-4 \Perp} \\
C_{-4 \underline{ }}\end{array}$ & $\begin{array}{l}C_{3 u} \\
C_{3 g} \\
C_{-3 u} \\
C_{-3 g}\end{array}$ & $\begin{array}{l}C_{4 g} \\
C_{4 u} \\
C_{-2 g} \\
C_{-2 g}\end{array}$ & $\begin{array}{l}C_{2 g} \\
C_{2 u} \\
C_{-4 g} \\
C_{-4 g}\end{array}$ & $\begin{array}{l}C_{3 g} \\
C_{3 u} \\
C_{-3 g} \\
C_{-3 u}\end{array}$ & $\begin{array}{l}C_{5 g} \\
C_{5 u} \\
C_{-1 g} \\
C_{-1 u}\end{array}$ & $\begin{array}{l}C_{1 s} \\
C_{1 u} \\
C_{-5 g} \\
C_{-5 u}\end{array}$ \\
\hline
\end{tabular}

(Pattern for higher $C_{ \pm n g, u}$ repeats)

\begin{tabular}{|c|c|c|c|c|c|c|c|c|}
\hline $\begin{array}{l}S_{1 g} \\
S_{1 u} \\
S_{-1 g} \\
S_{-1 u}\end{array}$ & $\begin{array}{l}S_{3 u} \\
S_{3 g} \\
S_{1 u} \\
S_{1 g}\end{array}$ & $\begin{array}{l}S_{-1 u} \\
S_{-1 g} \\
S_{-3 u} \\
S_{-3 g}\end{array}$ & $\begin{array}{l}S_{1 u} \\
S_{1 g} \\
S_{-1 u} \\
S_{-1 g}\end{array}$ & $\begin{array}{l}S_{3 g} \\
S_{3 u} \\
S_{1 g} \\
S_{1 u}\end{array}$ & $\begin{array}{l}S_{-1 g} \\
S_{-1 u} \\
S_{-3 g} \\
S_{-3 u}\end{array}$ & $\begin{array}{l}S_{1 g} \\
S_{1 u} \\
S_{-1 s} \\
S_{-1 u}\end{array}$ & $\begin{array}{l}S_{5 g} \\
S_{5 u} \\
S_{3 g} \\
S_{3 u}\end{array}$ & $\begin{array}{l}S_{-3 g} \\
S_{-3 u} \\
S_{-5 \xi} \\
S_{-5 u}\end{array}$ \\
\hline $\begin{array}{l}S_{3 g} \\
S_{3 u} \\
S_{-3 g} \\
S_{-3 u}\end{array}$ & $\begin{array}{l}S_{5 u} \\
S_{5 g} \\
S_{-1 u} \\
S_{-1 g}\end{array}$ & $\begin{array}{l}S_{1 u} \\
S_{1 g} \\
S_{-5 u} \\
S_{-5 g}\end{array}$ & $\begin{array}{l}S_{3 u} \\
S_{3 g} \\
S_{-3 u} \\
S_{-3 g}\end{array}$ & $\begin{array}{l}S_{5 g} \\
S_{5 u} \\
S_{-1 g} \\
S_{-1 u}\end{array}$ & $\begin{array}{l}S_{1 g} \\
S_{1 u} \\
S_{-5 g} \\
S_{-5 u}\end{array}$ & $\begin{array}{l}S_{3 g} \\
S_{3 u} \\
S_{-3 g} \\
S_{-3 u}\end{array}$ & $\begin{array}{l}S_{7 g} \\
S_{7 u} \\
S_{1 g} \\
S_{1 u}\end{array}$ & $\begin{array}{l}S_{-1 g} \\
S_{-1 u} \\
S_{-7 g} \\
S_{-7 u}\end{array}$ \\
\hline $\begin{array}{l}S_{5 g} \\
S_{5 u} \\
S_{-5 g} \\
S_{-5 u}\end{array}$ & $\begin{array}{l}S_{7 u} \\
S_{7 g} \\
S_{-3 u} \\
S_{-3 g}\end{array}$ & $\begin{array}{l}S_{3 u} \\
S_{3 g} \\
S_{-7 u} \\
S_{-7 g}\end{array}$ & $\begin{array}{l}S_{5 u} \\
S_{5 g} \\
S_{-5 u} \\
S_{-5 g}\end{array}$ & $\begin{array}{l}S_{7 g} \\
S_{7 u} \\
S_{-3 g} \\
S_{-3 u}\end{array}$ & $\begin{array}{l}S_{3 g} \\
S_{3 u} \\
S_{-7 g} \\
S_{-7 u}\end{array}$ & $\begin{array}{l}S_{58} \\
S_{5 u} \\
S_{-58} \\
S_{-5 u}\end{array}$ & $\begin{array}{l}S_{9 g} \\
S_{9 u} \\
S_{-1 g} \\
S_{-1 u}\end{array}$ & $\begin{array}{l}S_{1 g} \\
S_{1 u} \\
S_{-9 g} \\
S_{-9 u}\end{array}$ \\
\hline
\end{tabular}

(Pattern for higher $S_{ \pm n g, u}$ repeats)

\begin{tabular}{|c|c|c|c|c|c|c|c|c|}
\hline $\mathrm{C}_{6 h}$ & $z$ & $x+i y$ & $x-i y$ & $L_{z}, Z^{2}$ & $\begin{array}{l}L_{x}+i L_{y} \\
(x+i y) z\end{array}$ & $\begin{array}{l}L_{x}-i L_{y} \\
(x-i y) z\end{array}$ & $(x+i y)^{2}$ & $(x-i y)^{2}$ \\
\hline $\begin{array}{l}A_{g} \\
A_{u}\end{array}$ & $\begin{array}{l}A_{u} \\
A_{g}\end{array}$ & $\begin{array}{l}C_{1 u} \\
C_{1 g}\end{array}$ & $\begin{array}{l}C_{5 u} \\
C_{5 g}\end{array}$ & $\begin{array}{l}A_{g} \\
A_{u}\end{array}$ & $\begin{array}{l}C_{1 g} \\
C_{1 u}\end{array}$ & $\begin{array}{l}C_{5 g} \\
C_{5 u}\end{array}$ & $\begin{array}{l}C_{2 z} \\
C_{2 u}\end{array}$ & $\begin{array}{l}C_{4 g} \\
C_{4 u}\end{array}$ \\
\hline $\begin{array}{l}C_{1 g} \\
C_{1 u} \\
C_{2 g} \\
C_{2 u}\end{array}$ & $\begin{array}{l}C_{1 u} \\
C_{1 g} \\
C_{2 u} \\
C_{2 g}\end{array}$ & $\begin{array}{l}C_{2 u} \\
C_{2 g} \\
B_{u} \\
B_{g}\end{array}$ & $\begin{array}{l}A_{u} \\
A_{g} \\
C_{1 u} \\
C_{1 g}\end{array}$ & $\begin{array}{l}C_{1 g} \\
C_{1 u} \\
C_{2 g} \\
C_{2 u}\end{array}$ & $\begin{array}{l}C_{2 g} \\
C_{2 u} \\
B_{g} \\
B_{u}\end{array}$ & $\begin{array}{l}A_{g} \\
A_{u} \\
C_{1 g} \\
C_{1 u}\end{array}$ & $\begin{array}{l}B_{g} \\
B_{u} \\
C_{4 g} \\
C_{4 u}\end{array}$ & $\begin{array}{l}C_{5 g} \\
C_{5 u} \\
A_{g} \\
A_{u}\end{array}$ \\
\hline $\begin{array}{l}B_{s} \\
B_{u}\end{array}$ & $\begin{array}{l}B_{u} \\
B_{g}\end{array}$ & $\begin{array}{l}C_{4 u} \\
C_{4 g}\end{array}$ & $\begin{array}{l}C_{2 u} \\
C_{2 g}\end{array}$ & $\begin{array}{l}B_{g} \\
B_{u}\end{array}$ & $\begin{array}{l}C_{4 g} \\
C_{4 u}\end{array}$ & $\begin{array}{l}C_{2 g} \\
C_{2 u}\end{array}$ & $\begin{array}{l}C_{5 g} \\
C_{5 u}\end{array}$ & $\begin{array}{l}C_{1 g} \\
C_{1 u}\end{array}$ \\
\hline $\begin{array}{l}C_{4 g} \\
C_{4 u} \\
C_{5 g} \\
C_{5 u}\end{array}$ & $\begin{array}{l}C_{4 u} \\
C_{4 g} \\
C_{5 u} \\
C_{5 g}\end{array}$ & $\begin{array}{l}C_{5 u} \\
C_{5 g} \\
A_{u} \\
A_{g}\end{array}$ & $\begin{array}{l}B_{u} \\
B_{g} \\
C_{4 u} \\
C_{4 g}\end{array}$ & $\begin{array}{l}C_{4 g} \\
C_{4 u} \\
C_{5 g} \\
C_{5 u}\end{array}$ & $\begin{array}{l}C_{5 g} \\
C_{5 u} \\
A_{g} \\
A_{u}\end{array}$ & $\begin{array}{l}B_{g} \\
B_{u} \\
C_{4 g} \\
C_{4 u}\end{array}$ & $\begin{array}{l}A_{g} \\
A_{u} \\
C_{1 g} \\
C_{1 u}\end{array}$ & $\begin{array}{l}C_{2 g} \\
C_{2 u} \\
B_{g} \\
B_{u}\end{array}$ \\
\hline $\begin{array}{l}S_{1 g} \\
S_{1 u} \\
S_{3 g} \\
S_{3 u}\end{array}$ & $\begin{array}{l}S_{1 u} \\
S_{1 g} \\
S_{3 u} \\
S_{3 g}\end{array}$ & $\begin{array}{l}S_{3 u} \\
S_{3 g} \\
S_{5 u} \\
S_{5 g}\end{array}$ & $\begin{array}{l}S_{11 u} \\
S_{11 g} \\
S_{1 u} \\
S_{1 g}\end{array}$ & $\begin{array}{l}S_{1 g} \\
S_{1 u} \\
S_{3 g} \\
S_{3 u}\end{array}$ & $\begin{array}{l}S_{3 g} \\
S_{3 u} \\
S_{5 g} \\
S_{5 u}\end{array}$ & $\begin{array}{l}S_{11 g} \\
S_{11 u} \\
S_{1 g} \\
S_{1 u}\end{array}$ & $\begin{array}{l}S_{5 g} \\
S_{5 u} \\
S_{7 g} \\
S_{7 u}\end{array}$ & $\begin{array}{l}S_{9 g} \\
S_{9 u} \\
S_{11 g} \\
S_{11 u}\end{array}$ \\
\hline $\begin{array}{l}S_{5 g} \\
S_{5 u} \\
S_{7 g} \\
S_{7 u}\end{array}$ & $\begin{array}{l}S_{5 u} \\
S_{5 g} \\
S_{7 u} \\
S_{78}\end{array}$ & $\begin{array}{l}S_{7 u} \\
S_{7 g} \\
S_{9 u} \\
S_{9 g}\end{array}$ & $\begin{array}{l}S_{3 u} \\
S_{3 g} \\
S_{5 u} \\
S_{5 g}\end{array}$ & $\begin{array}{l}S_{5 g} \\
S_{5 u} \\
S_{7 g} \\
S_{7 u}\end{array}$ & $\begin{array}{l}S_{7 g} \\
S_{7 u} \\
S_{9 g} \\
S_{9 u}\end{array}$ & $\begin{array}{l}S_{3 g} \\
S_{3 u} \\
S_{5 g} \\
S_{5 u}\end{array}$ & $\begin{array}{l}S_{9 g} \\
S_{9 u} \\
S_{11 g} \\
S_{11 u}\end{array}$ & $\begin{array}{l}S_{1 g} \\
S_{1 u} \\
S_{3 g} \\
S_{3 u}\end{array}$ \\
\hline $\begin{array}{l}S_{9 g} \\
S_{9 u} \\
S_{11 g} \\
S_{11 u}\end{array}$ & $\begin{array}{l}S_{9 u} \\
S_{9 g} \\
S_{11 u} \\
S_{11 g}\end{array}$ & $\begin{array}{l}S_{11 u} \\
S_{11 g} \\
S_{1 u} \\
S_{1 g}\end{array}$ & $\begin{array}{l}S_{7 u} \\
S_{7 g} \\
S_{9 u} \\
S_{9 g}\end{array}$ & $\begin{array}{l}S_{9 g} \\
S_{9 u} \\
S_{11 g} \\
S_{11 u}\end{array}$ & $\begin{array}{l}S_{11 g} \\
S_{11 u} \\
S_{1 g} \\
S_{1 u}\end{array}$ & $\begin{array}{l}S_{7 g} \\
S_{7 u} \\
S_{9 g} \\
S_{9 u}\end{array}$ & $\begin{array}{l}S_{1 g} \\
S_{1 u} \\
S_{3 g} \\
S_{3 u}\end{array}$ & $\begin{array}{l}S_{5 g} \\
S_{5 u} \\
S_{7 g} \\
S_{7 u}\end{array}$ \\
\hline
\end{tabular}


TABLE 13. Selection rules-Continued

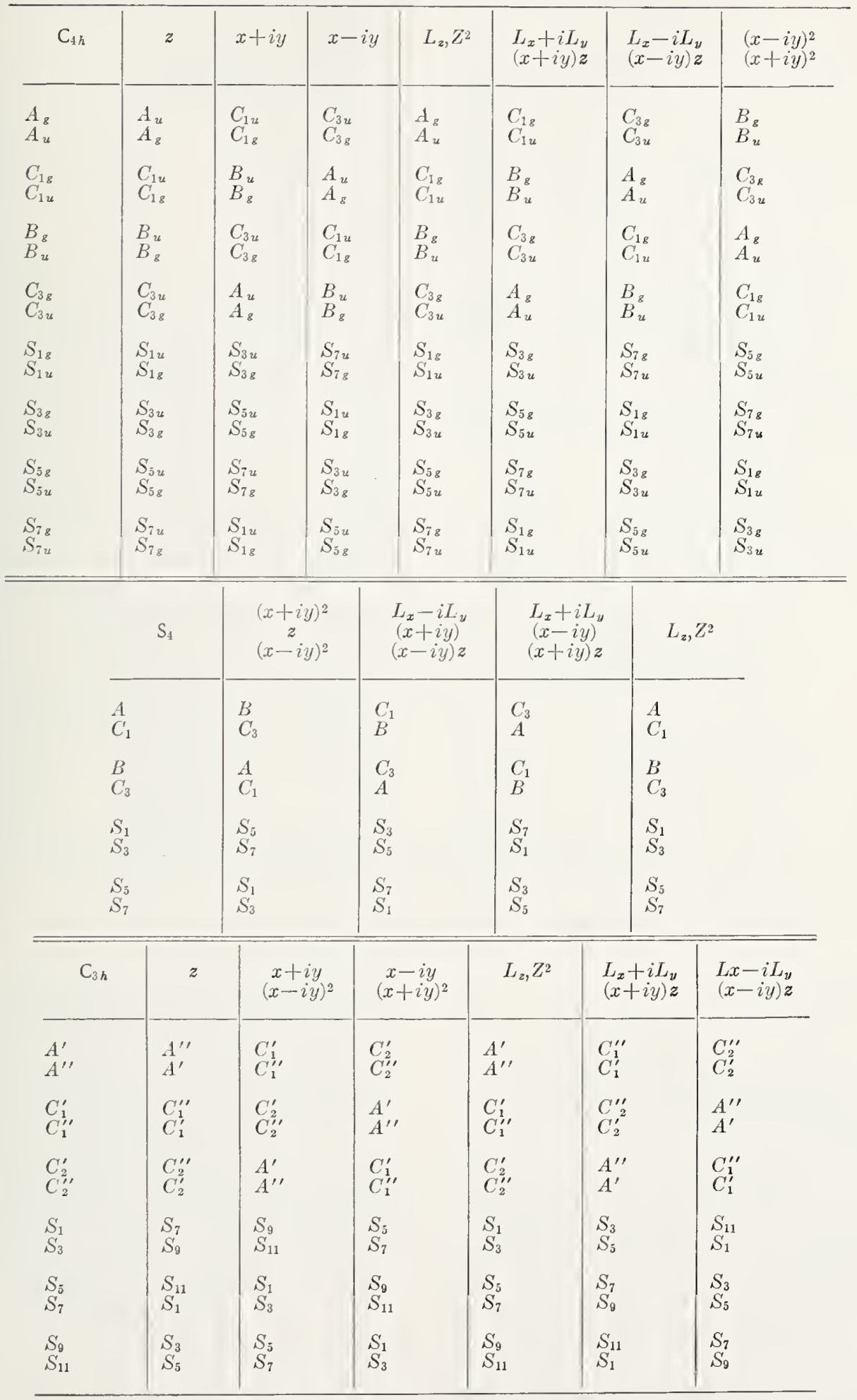


TABLE 13. Selection rules-Continued

\begin{tabular}{|c|c|c|c|c|}
\hline $\mathrm{C}_{2 h}$ & $x, y$ & $z$ & $\begin{array}{l}L_{x}, L_{y} \\
x \boldsymbol{z}, y z\end{array}$ & $\begin{array}{l}L_{z}, Z^{2} \\
x y, \rho^{2}\end{array}$ \\
\hline $\begin{array}{l}A_{g} \\
A_{u}\end{array}$ & $\begin{array}{l}B_{u} \\
B_{g}\end{array}$ & $\begin{array}{l}A_{u} \\
A_{g}\end{array}$ & $\begin{array}{l}B_{g} \\
B_{u}\end{array}$ & $\begin{array}{l}A_{g} \\
A_{u}\end{array}$ \\
\hline $\begin{array}{l}B_{g} \\
B_{u}\end{array}$ & $\begin{array}{l}A_{u} \\
A_{g}\end{array}$ & $\begin{array}{l}B_{u} \\
B_{g}\end{array}$ & $\begin{array}{l}A_{g} \\
A_{u}\end{array}$ & $\begin{array}{l}B_{g} \\
B_{u}\end{array}$ \\
\hline $\begin{array}{l}S_{1 g} \\
S_{1 u}\end{array}$ & $\begin{array}{l}S_{3 u} \\
S_{3 g}\end{array}$ & $\begin{array}{l}S_{1 u} \\
S_{1 g}\end{array}$ & $\begin{array}{l}S_{3 g} \\
S_{3 u}\end{array}$ & $\begin{array}{l}S_{1 s} \\
S_{1 u}\end{array}$ \\
\hline $\begin{array}{l}S_{3 g} \\
S_{3 u}\end{array}$ & $\begin{array}{l}S_{1 u} \\
S_{1 g}\end{array}$ & $\begin{array}{l}S_{3 u} \\
S_{3 R}\end{array}$ & $\begin{array}{l}S_{1 g} \\
S_{1 u}\end{array}$ & $\begin{array}{l}S_{38} \\
S_{3 u}\end{array}$ \\
\hline \multicolumn{2}{|c|}{$r$} & $L$ & $Z^{2}, \rho^{2}$ & $x \boldsymbol{z}, y \boldsymbol{z}, x y$ \\
\hline \multicolumn{2}{|l|}{$\begin{array}{l}T_{1 u} \\
T_{1 g}\end{array}$} & $\begin{array}{l}T_{1 g} \\
T_{1 u}\end{array}$ & $\begin{array}{l}E_{g} \\
E_{u}\end{array}$ & $\begin{array}{l}T_{2 g} \\
T_{2 u}\end{array}$ \\
\hline \multicolumn{2}{|l|}{$\begin{array}{l}T_{2 u} \\
T_{2 g}\end{array}$} & $\begin{array}{l}T_{2 g} \\
T_{2 u}\end{array}$ & $\begin{array}{l}E_{g} \\
E_{u}\end{array}$ & $\begin{array}{l}T_{1 g} \\
T_{1 u}\end{array}$ \\
\hline \multicolumn{2}{|c|}{$\begin{array}{l}T_{1 u} T_{2 u} \\
T_{1 g} T_{2 g}\end{array}$} & $\begin{array}{ll}T_{1 g} & T_{2 g} \\
T_{1 u} & T_{2 u}\end{array}$ & $\begin{array}{l}A_{1 g} A_{2 g} E_{g} \\
A_{1 u} A_{2 u} E_{u}\end{array}$ & $\begin{array}{ll}T_{18} & T_{28} \\
T_{1 u} & T_{2 u}\end{array}$ \\
\hline \multicolumn{2}{|c|}{$\begin{array}{l}A_{1 u} E_{u} T_{1 u} T_{2 u} \\
A_{1 g} E_{g} T_{1 g} T_{2 \xi}\end{array}$} & $\begin{array}{l}A_{1 g} E_{g} T_{1 g} T_{2 g} \\
A_{1 u} E_{u} T_{1 u} T_{2 u}\end{array}$ & $\begin{array}{ll}T_{1 g} & T_{2 g} \\
T_{1 u} & T_{2 u}\end{array}$ & $\begin{array}{llll}A_{2 g} & E_{g} & T_{1 g} & T_{2 g} \\
A_{2 u} & E_{u} & T_{1 u} & T_{2 u}\end{array}$ \\
\hline \multicolumn{2}{|c|}{ 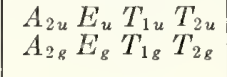 } & $\begin{array}{l}A_{2 g} E_{g} T_{1 z} T_{2 g} \\
A_{2 u} E_{u}^{\prime} T_{1 u} T_{2 u}\end{array}$ & $\begin{array}{l}T_{1 g} T_{2 g} \\
T_{1 u} T_{2 u}\end{array}$ & $\begin{array}{llll}A_{1 g} & E_{g} & T_{1 g} & T_{2 g} \\
A_{1 u} & E_{u} & T_{1 u} & T_{2 u}\end{array}$ \\
\hline \multicolumn{2}{|c|}{$\begin{array}{ll}D_{1 / 2 u} & D_{3 / 2 u} \\
D_{1 / 28} & D_{3 / 28}\end{array}$} & $\begin{array}{ll}D_{1 / 2 g} & D_{3 / 2 g} \\
D_{1 / 2 u} & D_{3 / 2 u}\end{array}$ & $\begin{array}{l}D_{3 / 2 g} \\
D_{3 / 2 u}\end{array}$ & $\begin{array}{ll}{ }_{2} S_{g} & D_{3 / 2 g} \\
{ }_{2} S_{u} & D_{3 / 2 u}\end{array}$ \\
\hline \multicolumn{2}{|c|}{$\begin{array}{ll}{ }_{2} S_{u} & D_{3 / 2 u} \\
{ }_{2} S_{g} & D_{3 / 2 g}\end{array}$} & $\begin{array}{ll}{ }_{2} S_{\mathrm{g}} & D_{3 / 2 g} \\
{ }_{2} S_{u} & D_{3 / 2 u}\end{array}$ & $\begin{array}{l}D_{3 / 2 g} \\
D_{3 / 2 u}\end{array}$ & $\begin{array}{ll}D_{1 / 2 g} & D_{3 / 2 g} \\
D_{1 / 2} u & D_{3 / 2 u}\end{array}$ \\
\hline \multicolumn{2}{|c|}{\begin{tabular}{|lll}
${ }_{2} S_{u}$ & $D_{1 / 2 u}$ & $D_{3 / 2 u}$ \\
$2 S_{g}$ & $D_{1 / 28}$ & $D_{3 / 28}$
\end{tabular}} & $\begin{array}{lll}{ }_{2} S_{g} & D_{1 / 2 g} & D_{3 / 2 g} \\
{ }_{2} S_{u} & D_{3 / 2 u} & D_{3 / 2 u}\end{array}$ & $\begin{array}{lll}{ }_{2} S_{g} & D_{1 / 2 g} & D_{3 / 2 g} \\
{ }_{2} S_{u} & D_{1 / 2 u} & D_{3 / 2 u}\end{array}$ & $\begin{array}{lll}{ }_{2} S_{g} & D_{1 / 2 g} & D_{3 / 2 g} \\
{ }_{2} S_{u} & D_{1 / 2 u} & D_{3 / 2 u}\end{array}$ \\
\hline
\end{tabular}

and the inverse relations

$\mathbf{i}=\sin \theta \cos \phi \mathbb{r}_{0}+\cos \theta \cos \phi \theta_{0}-\sin \phi \phi_{0}$

$\mathbf{j}=\sin \theta \sin \phi \mathbb{r}_{0}+\cos \theta \sin \phi \theta_{0}+\cos \phi \phi_{0}$

$\mathbf{k}=\cos \theta \mathbf{r}_{0}-\sin \theta \boldsymbol{\theta}_{0}$.

Let us further consider $\mathrm{A}$ as a plane polarized wave, incident toward the origin along the vector $\mathbf{r}_{0}\left[\mathbf{r}_{0}\right.$ points to the source of $\left.\AA\right]$, and having components along $\theta_{0}$ and $\phi_{0}$ perpendicular to the direction of propagation.

$$
\mathbf{A}=\left(A \cos \psi \theta_{0}+A \sin \psi \phi_{0}\right) \exp \mathbf{i}\left(\sigma\left[\mathbf{r} \cdot \mathbf{r}_{0}\right]+\omega t\right) .
$$

In the terminology of the Euler angles of section 3.2 , the plane polarized light is incident along the $\zeta$ axis with $\mathbf{A}$ parallel to the $\xi$ axis. At the origin,
$\mathrm{A}^{0}$ may be resolved into components as follows:

$$
\begin{aligned}
& A_{x}^{0}=A e^{i \omega t}(\cos \psi \cos \theta \cos \phi-\sin \psi \sin \phi) \\
& A_{y}^{0}=A e^{i \omega t}(\cos \psi \cos \theta \sin \phi+\sin \psi \cos \phi) \\
& A_{z}^{0}=A e^{i \omega t}(-\cos \psi \sin \theta) .
\end{aligned}
$$

The vector A changes in space only along the direction $\mathbf{r}_{0}$, and hence a variation along the $x$-axis, for example, will change $\mathbf{A}$ according to the component of the variation along $\mathbf{r}_{0}$. Formally,

$$
\frac{\partial \mathbf{A}}{\partial x}=\frac{\partial \mathbf{A}}{\partial\left[\mathbf{r} \cdot \mathbf{r}_{0}\right]} \cdot \frac{\partial\left[\mathbf{r} \cdot \mathbf{r}_{0}\right]}{\partial x}
$$

Letting

$$
\frac{\partial \mathbf{A}}{\partial\left[\mathbf{r} \cdot \mathbf{r}_{0}\right]}=\mathbf{A}^{\prime}
$$


Table 13. Selection rules-Continued

\begin{tabular}{|c|c|c|c|c|}
\hline $\mathrm{T}_{h}$ & $r$ & $\underset{x \boldsymbol{z}, y \boldsymbol{z}, x y}{L}$ & $Z^{2}+i \rho^{2}$ & $Z^{2}-i \rho$ \\
\hline$A_{g}$ & $\begin{array}{l}T_{u} \\
T_{\boldsymbol{g}}\end{array}$ & $\begin{array}{l}T_{g} \\
T_{u}\end{array}$ & $\begin{array}{l}C_{1 g} \\
C_{1 u}\end{array}$ & $\begin{array}{l}C_{2 g} \\
C_{2 u}\end{array}$ \\
\hline $\begin{array}{l}C_{18} \\
C_{1 u}\end{array}$ & $\begin{array}{l}T_{u} \\
T_{8}\end{array}$ & $\begin{array}{l}T_{g} \\
T_{u}\end{array}$ & $\begin{array}{l}C_{2 g} \\
C_{2 u}\end{array}$ & $\begin{array}{l}A_{g} \\
A_{u}\end{array}$ \\
\hline $\begin{array}{l}C_{2 g} \\
C_{2 u}\end{array}$ & $\begin{array}{l}T_{u} \\
T_{g}\end{array}$ & $\begin{array}{l}T_{g} \\
T_{u}\end{array}$ & $\begin{array}{l}A_{g} \\
A_{u}\end{array}$ & $\begin{array}{l}C_{1 g} \\
C_{1 u}\end{array}$ \\
\hline $\begin{array}{l}T_{g} \\
T_{u}\end{array}$ & $\begin{array}{l}A_{u} C_{1 u} C_{2 u} T_{u} \\
A_{g} C_{1 g} C_{2 g} T_{g}\end{array}$ & 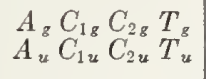 & $\begin{array}{l}T_{g} \\
T_{u}\end{array}$ & $\begin{array}{l}T_{g} \\
T_{u}\end{array}$ \\
\hline $\begin{array}{l}D_{1 / 28} \\
D_{3 / 2 u}\end{array}$ & $\begin{array}{l}D_{1 / 2 u} S_{1 u 2} S_{2 u} \\
D_{3 / 2 g} S_{1 g} S_{22} S_{2 g}\end{array}$ & $\begin{array}{l}D_{1 / 3 g}{ }_{2} S_{1 g}{ }_{2} S_{2 g} \\
D_{1 / 2 u} S_{1 u} S_{2 u}\end{array}$ & $\begin{array}{l}{ }_{2} S_{1 g} \\
2 S_{1 u}\end{array}$ & $\begin{array}{l}{ }_{2} S_{2 g} \\
{ }_{2} S_{2 u}\end{array}$ \\
\hline $\begin{array}{l}{ }_{2}^{2} S_{1 g} \\
{ }_{2} S_{1 u}\end{array}$ & $\begin{array}{l}D_{1 / 2 u} S_{1 u} S_{12} S_{2 u} \\
D_{3 / 2 z} S_{18} S_{2} S_{2 g}\end{array}$ & $\begin{array}{l}D_{1 / 2 g}{ }_{2} S_{1 g} S_{2} S_{2 g} \\
D_{3 / 2 u} S_{1 u 2} S_{2 u}\end{array}$ & $\begin{array}{l}{ }_{2} S_{2 g} \\
{ }_{2} S_{2 u}\end{array}$ & $\begin{array}{l}D_{1 / 3 g} \\
D_{1 / 2 u}\end{array}$ \\
\hline $\begin{array}{l}{ }_{2} S_{2 g} \\
{ }_{2} S_{2 u}\end{array}$ & $\begin{array}{l}D_{1 / 5 u} S_{2} S_{1 u} S_{2 u} \\
D_{1 / 3 g} S_{1 g} S_{2 g} S_{2 g}\end{array}$ & $\begin{array}{l}D_{1 / 2 g} S_{1 g} S_{2} S_{2 g} \\
D_{1 / 2 u 2} S_{1 u 2} S_{2 u}\end{array}$ & $\begin{array}{l}D_{1 / 28} \\
D_{1 / 24}\end{array}$ & $\begin{array}{l}{ }_{2} S_{1 g} \\
{ }_{2} S_{1 u}\end{array}$ \\
\hline $\mathrm{T}_{d}$ & $\stackrel{r}{x \boldsymbol{z}, y \boldsymbol{z}, x y}$ & $L$ & & $2, \rho^{2}$ \\
\hline $\begin{array}{l}A_{1} \\
A_{2}\end{array}$ & $\begin{array}{l}T_{2} \\
T_{1}\end{array}$ & $\begin{array}{l}T_{1} \\
T_{2}\end{array}$ & $\begin{array}{l}E \\
E\end{array}$ & \\
\hline $\begin{array}{c}E \\
T_{1}\end{array}$ & $\begin{array}{l}T_{1} T_{2} \\
A_{2} E T_{1} T_{2}\end{array}$ & 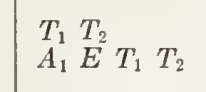 & $\begin{array}{l}A_{1} \\
T_{1}\end{array}$ & ${ }_{2}^{2} E$ \\
\hline $\begin{array}{r}T_{2} \\
D_{3 / 2}\end{array}$ & $\begin{array}{llll}A_{1} E & T_{1} & T_{2} \\
{ }_{2} S & D_{3 / 2}\end{array}$ & $\begin{array}{l}A_{2} E T_{1} T_{2} \\
D_{3 / 2} D_{3 / 2}\end{array}$ & $\begin{array}{l}T_{1} T \\
D_{3 / 2}\end{array}$ & \\
\hline$D_{3 / 2}^{2 S}$ & $\begin{array}{l}D_{1 / 2} D_{3 / 2} \\
D_{1 / 2} S^{2} D_{3 / 2}\end{array}$ & 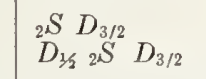 & $\begin{array}{l}D_{3 / 2} \\
D_{1 / 2}\end{array}$ & $S \quad D_{3 / 2}$ \\
\hline
\end{tabular}

we have

$$
\frac{\partial \mathbf{A}}{\partial x}=\mathbf{A}^{\prime}\left(\mathbf{i} \cdot \mathbf{r}_{0}\right), \frac{\partial \mathbf{A}}{\partial y}=\mathbf{A}^{\prime}\left(\mathbf{j} \cdot \mathbf{r}_{0}\right), \frac{\partial \mathbf{A}}{\partial z}=\mathbf{A}^{\prime}\left(\mathbf{k} \cdot \mathbf{r}_{0}\right)
$$

Obviously, the resolution of $\mathbf{A}^{\prime}$ into components is parallel to that of $\mathbf{A}$, so we obtain the following 9 terms involving the derivatives of $\mathbf{A}$ :

$\frac{\partial A_{x}}{\partial x}=A^{\prime}(\cos \psi \cos \theta \cos \phi-\sin \psi \sin \phi)(\sin \theta \cos \phi)$ $\frac{\partial A_{x}}{\partial y}=A^{\prime}(\cos \psi \cos \theta \cos \phi-\sin \psi \sin \phi)(\sin \theta \sin \phi)$ $\frac{\partial A_{x}}{\partial z}=A^{\prime}(\cos \psi \cos \theta \cos \phi-\sin \psi \sin \phi)(\cos \theta)$ $\frac{\partial A_{y}}{\partial x}=A^{\prime}(\cos \psi \cos \theta \sin \phi+\sin \psi \cos \phi)(\sin \theta \cos \phi)$ $\frac{\partial A_{y}}{\partial y}=A^{\prime}(\cos \psi \cos \theta \sin \phi+\sin \psi \cos \phi)(\sin \theta \sin \phi)$

$\frac{\partial A_{y}}{\partial z}=A^{\prime}(\cos \psi \cos \theta \sin \phi+\sin \psi \cos \phi)(\cos \theta)$

$\frac{\partial A_{2}}{\partial x}=A^{\prime}(-\cos \psi \sin \theta)(\sin \theta \cos \phi)$

$\frac{\partial A_{z}}{\partial y}=A^{\prime}(-\cos \psi \sin \theta)(\sin \theta \sin \phi)$

$\frac{\partial A_{2}}{\partial z}=A^{\prime}(-\cos \psi \sin \theta)(\cos \theta)$.

These may now be combined as previously defined (page 55) to yield

$A_{x x}=\frac{1}{2} A^{\prime}\left(\cos \psi \sin 2 \theta \cos ^{2} \phi-\sin \psi \sin \theta \sin 2 \phi\right)$

$A_{y y}=\frac{1}{2} A^{\prime}\left(\cos \psi \sin 2 \theta \sin ^{2} \phi+\sin \psi \sin \theta \sin 2 \phi\right)$

$A_{z z}=\frac{1}{2} A^{\prime}(-\cos \psi \sin 2 \theta)$

$A_{x y}=\frac{1}{2} A^{\prime}(\cos \psi \sin 2 \theta \sin 2 \phi+2 \sin \psi \sin \theta \cos 2 \phi)$

$A_{x z}=A^{\prime}(\cos \psi \cos 2 \theta \cos \phi-\sin \psi \cos \theta \sin \phi)$

$A_{y z}=A^{\prime}(\cos \psi \cos 2 \theta \sin \phi+\sin \psi \cos \theta \cos \phi)$

$B_{x}=-A^{\prime}(\cos \psi \sin \phi+\sin \psi \cos \theta \cos \phi)$

$B_{y}=A^{\prime}(\cos \psi \cos \phi-\sin \psi \cos \theta \sin \phi)$

$B_{2}=A^{\prime}(\sin \psi \sin \theta)$.

We may note that $A_{x x}+A_{y y}+A_{z z}$ is in fact zero, and that the angular factors for the components of $\mathrm{B}$ are the same as those for $\mathbf{A}$ with a rotation of $90^{\circ}$ in $\psi$, showing the mutual perpendicularity of $\mathbf{B}$ and A. In order to obtain our final five quadrupole components, there is a question of normalization which must be considered. Strictly speaking, the appropriate "base vectors" for the quadrupole components are the normalized tesseral harmonics of table 2 for $N=2$. Since we shall be interested in relative intensities, we may omit a common factor, which we shall here choose to be $\frac{\sqrt{15}}{2 \sqrt{\pi}}$. With the omission of this factor, $x y, x z$, and $y z$ will still be the proper basis, but for the other 2, where we have heretofore used $x^{2}-y^{2}$ and $2 z^{2}$ 
$-x^{2}-y^{2}$, we must now include factors of $\frac{1}{2}$ and $\frac{1}{2 \sqrt{3}}$ respectively, so the balance of the term with these two bases will become multiplied by the reciprocals of these factors. We shall continue to denote these two renormalized bases as $\rho^{2}$ and $Z^{2}$.

Finally, we must consider the resolution of the multipole interactions into the (complex) base vectors appropriate to the group $C_{\infty h}$ and its subgroups with complex reps. The terms in $z, L_{z}$, and $Z^{2}$ will be unaffected by this change. In lieu of $x$ and $y$, we shall use $\frac{1}{\sqrt{2}}(x+i y)$ and $\frac{1}{\sqrt{2}}(x-i y)$, for $L_{x}$ and $L_{y}$ a similar combination, and for $\rho^{2}$ and $x y, \frac{1}{\sqrt{2}}\left(\rho^{2}+i x y\right)$ and $\frac{1}{\sqrt{2}}\left(\rho^{2}-i x y\right)$. This requires the use of the following linear combinations of the previous terms:

$$
\begin{array}{rlrl}
A_{+}^{0} & =\frac{\sqrt{2}}{2}\left(A_{x}^{0}-i A_{y}^{0}\right) & A_{-}^{0}=\frac{\sqrt{2}}{2}\left(A_{x}^{0}+i A_{y}^{0}\right) \\
B_{+}^{0}=\frac{\sqrt{2}}{2}\left(B_{x}^{0-i} B_{y}^{0}\right) & B_{-}^{0}=\frac{\sqrt{2}}{2}\left(B_{x}^{0}+i B_{y}^{0}\right) \\
A_{z+}=\frac{\sqrt{2}}{2}\left(A_{x z}-i A_{y z}\right) & A_{z-}=\frac{\sqrt{2}}{2}\left(A_{x z}+i A_{y z}\right) \\
A_{2+}=\frac{\sqrt{2}}{2}\left(A_{\rho 2}-i A_{x y}\right) & A_{2-}=\frac{\sqrt{2}}{2}\left(A_{\rho^{2}}+i A_{x y}\right) .
\end{array}
$$

These are all summarized in table 14.

Let us now consider some of the conclusions of this analysis. We shall discuss in detail six cases.

1. Only one component of the eleven considered is effective in producing the transition. In this case, the rate of absorption will be proportional to the Hermitian square of the matrix element, and hence proportional to the Hermitian square of the appropriate angular dependence factor of table 14. Such a case is exemplified by the $B_{1 g}-B_{2 g}$ transition in $\mathrm{D}_{4 h}$, which are joined only by $L_{z}$. The angular dependence is thus $(\sin \psi \sin \theta)^{2}$.

2. 'Two components only of the eleven join the states, these two belonging to a doubly degenerate rep and consequently arising from the same type of multipole interaction (e.g., in $D_{6 h}, A_{1 g}$ is joined to $E_{2 g}$ by both $x y$ and $\rho^{2}$. Then the total matrix element will involve the sum of the two angular factors for the two multipole components, and the angular dependence will be proportional to the Hermitian square of the sum of these angular factors.

3. Two components only join the states involved, arising from different multipole interactions (e.g., $A_{1}$ of $\mathrm{C}_{60}$ will join itself by either $z$ or $Z^{2}$ ). The matrix element will then include the sum of the angular factors for the two components, but one will be multiplied by a factor " $q$ " determined by the numerical ratio of the strength of
TABLE 14. Angular dependence of multipole components

1. Electric Dipole

$A_{z}^{0}=A(-\cos \psi \sin \theta)$

$A_{x}^{0}=A(\cos \psi \cos \theta \cos \phi-\sin \psi \sin \phi)$

$A_{y}^{0}=A(\cos \psi \cos \theta \sin \phi+\sin \psi \cos \phi)$

$A_{+}^{0}=A\left(\frac{\sqrt{2}}{2}\right)(\cos \psi \cos \theta-i \sin \psi) e^{-i \phi}$
$A_{-}^{0}=A\left(\frac{\sqrt{2}}{2}\right)(\cos \psi \cos \theta+i \sin \psi) e^{i \phi}$

2. Magnetic Dipole

$B_{z}=A^{\prime}(\sin \psi \sin \theta)$

$B_{x}=A^{\prime}(-\cos \psi \sin \phi-\sin \psi \cos \theta \cos \phi)$

$B_{u}=A^{\prime}(\cos \psi \cos \phi-\sin \psi \cos \theta \sin \phi)$

$B_{+}=A^{\prime}\left(\frac{\sqrt{2}}{2}\right)(-\sin \psi \cos \theta-i \cos \psi) e^{-i \phi}$

$B_{-}=A^{\prime}\left(\frac{\sqrt{2}}{2}\right)(-\sin \psi \cos \theta+i \cos \psi) e^{i \phi}$

3. Electric Quadrupole

$A_{z 2}=A^{\prime}\left(\frac{\sqrt{3}}{2}\right)(-\cos \psi \sin 2 \theta)$

$A_{x z}=A^{\prime}(\cos \psi \cos 2 \theta \cos \phi-\sin \psi \cos \theta \sin \phi)$

$A_{y z}=A^{\prime}(\cos \psi \cos 2 \theta \sin \phi+\sin \psi \cos \theta \cos \phi)$

$A \rho^{2}=\frac{1}{2} A^{\prime}(\cos \psi \sin 2 \theta \cos 2 \phi-2 \sin \psi \sin \theta \sin 2 \phi)$

$A_{x y}=\frac{1}{2} A^{\prime}(\cos \psi \sin 2 \theta \sin 2 \phi+2 \sin \psi \sin \theta \cos 2 \phi)$

$A_{z+}=A^{\prime}\left(\frac{\sqrt{2}}{2}\right)(\cos \psi \cos 2 \theta-i \sin \psi \cos \theta) e^{-i \phi}$

$A_{z_{-}-}=A^{\prime}\left(\frac{\sqrt{2}}{2}\right)(\cos \psi \cos 2 \theta+i \sin \psi \cos \theta) e^{i \phi}$

$A_{2+}=A^{\prime}\left(\frac{\sqrt{2}}{4}\right)(\cos \psi \sin 2 \theta-2 i \sin \psi \sin \theta) e^{-2 i \phi}$

$A_{2-}=A^{\prime}\left(\frac{\sqrt{2}}{4}\right)(\cos \psi \sin 2 \theta+2 i \sin \psi \sin \theta) c^{2 i \phi}$

the two types of interaction, and is fixed by explicit calculations and not by general symmetry considerations. The angular variation will then be proportional to the Hermitian square of this sum, and will contain $q$ and $q^{2}$. If one type of interaction is very much stronger than the other, then $q$ will be small and it may be neglected. In the present problem of crystal spectra, this is not necessarily the case, and each case must be examined in detail.

4. Two components join the states in question, arising from the same multipole interaction, but the components belong to one-dimensional reps (as distinguished from (2)). For example, in $D_{2 h}, A_{2 g}$ is joined to itself by both $Z^{2}$ and $\rho^{2}$. The details here are very similar to case (3). The matrix element will include the sum of the angular factors. However, in contrast to (1), the term $Z^{2}$ is not related by symmetry to $\rho^{2}$, so one angular factor will again be multiplied by a factor $q$, expressing the relative strength of the interaction, and the Hermitian square will again contain both $q$ and $q^{2}$. Generally, one may only expect that $q$ will in this case be nearer 1 than in case (3).

5. Only one component joins the states, but another component joins another pair of states, both of which are respectively degenerate with the first pair. This is a frequent situation in 
$\mathrm{C}_{\infty h}$ and its subgroups. Here the transitions proceed independently, and the total absorption will be proportional to the sum of the Hermitian squares of the angular factors, rather than to the Hermitian square of the sum as in previous cases.

6. If the states are joined by three components, all belonging to the same triply degenerate rep, as is the case for both electric and magnetic dipole transitions in the cubic groups, there are no polarization effects.

We shall not discuss in detail more complicated cases. In general, the angular dependence will include factors expressing the relative strengths of the transitions arising from different dipole interactions, as well as from nondegenerate interactions of the same order. In specific cases, these relative strengths may be computed from the methods of the following section.

\section{Matrix Elements}

\subsection{The Reduced Matrix Element}

We now turn our attention to the procedure alternate to that which has occupied our attention in the preceding two sections, namely, the calculation of the matrix elements of the perturbing potentials of the crystal field and their interpretation. These potentials rere discussed in detail in section 2. Te have already touched upon certain general features of the problem in section 4.3-we must now examine this situation in more detail, and also determine the simplifying relationships which exist among the nonvanishing elements.

So far in our discussion of the transformation properties of the several wave functions belonging to a degenerate rep of a group, we have emphasized the equivalence of these several wave functions, as well as the properties of the rep (notably the character and class structure) which were invariant under a unitary transformation. We shall now depart from this procedure. We are now interested in picking out a particular set of linearly independent wave functions, in terms of which specific matrices representing the operations of the group may be written down. This is equivalent to the introduction of a specific system of base vectors in ordinary vector analysis.

Since we start with the wave functions of the free ion, described by the quantum number $J$, with $2 J+1$ linearly independent wave functions, we select for our basis, functions distinguished by the quantum number $M$ (or $M_{J}$ will sometimes be used for clarity) where $M=J, J-1, J-2, \ldots$ $-J$, and such that the matrix representing rotations about the $z$-axis is diagonal with elements $M e^{i M \phi}$. We have seen from the preceding section that wave functions belonging to different reps (here, with different $J$ values) are orthogonal-we see that these $2 J+1$ functions themselves belong to different reps of the group $C_{\infty}$, and hence are mutually orthogonal within a given $J$. These wave functions we can write as $|\alpha J M\rangle$ (in the notation of Dirac), where $\alpha$ represents all quantum numbers other than $J$ and $M$. To emphasize that such a state is olle component of a $2 J+1$ dimension rep of the rotation group, we may also write in the form $(\alpha /)|J M\rangle$. A different choice of axes will leave the (scalar) portion of the function unaltered, but will convert the normalized com- ponent of the rep $|J M\rangle$ into a combination of the same $J$ and different $M$.

For these calculations, it will be convenient to use the complex potentials $Y_{\nu}^{\mu}$ (2.9) rather than the real $C_{\nu}^{\mu}$ and $S_{\nu}^{\mu}(2.5)$ as our perturbation. We note here that these perturbing potentials actually act upon the space coordinates of the individual electrons, so the total perturbation is a sum of such over all electrons. Clearly, if these potentials obey a certain transformation law in the coordinates of one electron, their sum will similarly transform when all the coordinates of all electrons undergo the simultaneous transformations implied by the quantum number $\mathcal{J}$, or when only the space coordinates of all electrons undergo the simultaneous transformation implied by the quantum number $L$. Our problem has therefore become that of computing the sum of terms of the form

$$
\left\langle\alpha^{\prime} J^{\prime} M^{\prime}\left|r^{\nu} Y_{\nu}^{\mu}\right| \alpha J M\right\rangle
$$

We shall for the time being disregard the factor $r^{\nu}$.

According to section 4.3, we know that the matrix element will vanish unless $J^{\prime}$ is one of the values $J^{\prime \prime}$ arising from the reduction of the product $D_{J} \times D_{\nu}$, namely, $J+\nu, J+\nu-1, \ldots|J-\nu|$. Let us expand the product $Y_{\nu}^{\mu} \mid \alpha J M>$ into a sum of terms

$$
\sum_{M^{\prime \prime}} C_{M \mu M^{\prime \prime}}^{J \nu J^{\prime \prime}}\left(\Upsilon_{\nu}|| \alpha J\right) \mid J^{\prime \prime} M^{\prime \prime}>
$$

where the $C$-coefficients are the Wigner coefficients to be discussed further in section 5.2, the term $\left(Y_{\nu} \| \alpha J\right)$ represents the portion of the product invariant under a rotation of the axes, and $\left|J^{\prime \prime} M^{\prime \prime}\right\rangle$ represents the $M^{\prime \prime}-$ th component of the normalized rep $D_{J^{\prime \prime}}$ of the rotation group. We now know that the remaining term of (5.1), $\left\langle\alpha^{\prime} J^{\prime} M^{\prime}\left|=\left(\alpha^{\prime} J^{\prime}\right)<J^{\prime} M^{\prime}\right|\right.$ is orthogonal to all states except $\left|J^{\prime} M^{\prime}\right\rangle$, i.e.,

$$
\left\langle J^{\prime} M^{\prime} \mid J^{\prime \prime} M^{\prime \prime}\right\rangle=\delta_{J^{\prime} J^{\prime \prime}} \delta_{M H^{\prime} M \prime \prime}
$$

so we obtain, from (5.2) and (5.3),

$$
\left\langle\alpha^{\prime} J^{\prime} M^{\prime}\left|Y_{\nu}^{\mu}\right| \alpha J M\right\rangle=C_{M \mu M Y^{\prime}}^{J^{\prime}{ }^{\prime}}\left(\alpha^{\prime} J^{\prime}\left\|Y_{\nu}\right\| \alpha J\right) \text {. }
$$

In this expression, parity is included in the $\alpha, \alpha^{\prime}$. If $\nu$ is even, $\alpha$ and $\alpha^{\prime}$ must be of the same parity; if odd, of opposite parity. 
The importance of this step is that we have placed into the term $C_{M \mu M^{\prime}}^{J^{\prime}}$ the dependence of the matrix element on $M, M^{\prime}$, and $\mu$, and the "reduced matrix element" $\left(\alpha^{\prime} J^{\prime}\left\|Y_{\nu}\right\| \alpha \widetilde{J}\right)$ contains those portions of the interaction not dependent on these quantum numbers. If the matrix element is evaluated for one particular $M, M^{\prime}$, and $\mu$, then the reduced matrix element can be obtained, and from it the matrix element for any other $M, M^{\prime}$, and $\mu$. This relation was initially given by Eckart (1930) and again by Wigner (1931) and Racah (1942a). Therc is some divergence in the literature on normalization and phase factors for these reduced matrix elements. We have here followed Eckart, Wigner, Rose, and others, while Racah, Biedenharn (1952), and Simon et al. (1954) have used as reduced matrix element a quantity greater by a factor $\sqrt{2 J^{\prime}+1}$.

Eckart and Wigner have based their derivation of this result on the ideas of the representations of the rotation group, while Racah has developed the algebraic application of the angular momentum operators $J_{x}, J_{y}$, and $J_{z}$, which may be interpreted as differential rotations about the $x, y$, or $z$-axes, respectively. Since the vectorial character of a vector operator $\mathbb{T}$ with components $T^{7}, T^{0}, T^{-1}$ is fixed by the general commutation rules $\left[\dot{J}_{z}, T^{n}\right]=$ $n T^{n}$ and $\left[J_{x} \pm i J_{y}, T^{n}\right]=\sqrt{(1 \mp n)(1 \pm n+1)} T^{n \pm 1}, \mathrm{a}$ generalized irreducible tensor operator of degree F. has been defined as one having $2 k+1$ components and satisfying the commutation rules $\left[J_{z}, T_{k}^{n}\right]=n T_{k}^{n}$ and $\left[J_{x} \pm i J_{y}, T_{k}^{n}\right]=\sqrt{(k \mp n)(k \pm n+1)}$ $T_{k}^{n \pm 1}$. Our quantity $Y_{y}^{\mu}$ is a specific example of such an operator.

Stevens (1952) has emphasized a geometrical interpretation of this result, and has given what amounts to a technique for calculating certain of the Wigner coefficients in the special case $J^{\prime}=J$. The behavior under rotation of any quantity which may be expressed in terms of variously oriented coordinate systems, may be described by one or more components along a set of mutually orthogonal base vectors in an infinite number of dimensions. Subspaces of dimension 1, 2, 3, $2 J+1$ determine a particular $D_{J}$, while the individual vectors within the subspaces correspond to the various rows of the $D_{J}$. A product of two components, each of which lies along one of these base vectors (such as the $Y_{\nu}^{\mu} \mid \alpha d M>$ being considered) may itself be resolved into components by means of the Wigner coefficients; all save the one parallel to the third quantity $\left\langle\alpha^{\prime} J^{\prime} M^{\prime}\right|$ will vanish in the matrix element. Stevens also pointed out that it is possible to form irreducible tensors $\coprod_{\nu}^{\mu}$ from the noncommuting components of $J$ by taking the $2 \nu+1$ totally symmetric products of degree $\nu$. This is analogous to the process we have already used several times in connection with the reduction of the general product of two vectors. We then have

$$
\left\langle\alpha J M^{\prime}\left|\bigcup_{\nu}^{\mu}\right| \alpha J M>=C_{M \mu}^{J \nu} M^{\prime}\left(J\|\|_{\nu} \|_{,} J\right)\right.
$$

since this reduced matrix element is diagonal in both $\alpha$ and $J$, and independent of $\alpha$. These matrix elements may themselves be calculated, giving a set of numbers proportional to the Wigner coefficients. Certain tables based on this procedure have been given by Stevens (1952), by Elliott and Stevens (1953a), and by Judd (1955).

\subsection{Wigner Coefricients}

The Wigner coefficients $C_{M_{1}^{1} M_{2}^{2}}^{J_{2}}$, frequently written $\left(J_{1} J_{2} J M \mid J_{1} J_{2} M_{1} M_{2}\right)$ are the elements of the unitary matrix $\mathrm{C}$ which will reduce the $\left(2 J_{1}+1\right) \times$ $\left(2 \bar{J}_{2}+1\right)$ dimensional direct product of the components of $D_{J_{1}}$ and $D_{J_{2}}$ and which will provide the proper linear combinations of the products of these components $\left|J_{1} M_{1}\right\rangle$ and $\left|J_{2} M_{2}\right\rangle$ appropriate to the reduced component $|J M\rangle$. The columns of the $\mathrm{C}$ matrix are distinguished by the double indices $\left(M_{1}, M_{2}\right)$, while the rows bear the indices $(J, M)$. The phase conventions implied in (2.8) ensure that the matrix elements may be chosen to be real. The inverse $\mathbf{C}^{-1}$ is merely the transposed matrix. The orthogonality relations for a real unitary (= orthogonal) matrix then give us

$$
\begin{aligned}
& \sum_{M_{1}, M_{2}}\left(J_{1} J_{2} M_{1} M_{2} \mid J_{1} J_{2} J M\right)\left(J_{1} J_{2} M_{1} M_{2} \mid J_{1} J_{2} J^{\prime} M^{\prime}\right) \\
& =\delta_{J J^{\prime}} \delta_{M M^{\prime}} \\
& \sum_{J, M}\left(J_{1} J_{2} M_{1} M_{2} \mid J_{1} J_{2} J M\right)\left(J_{1} J_{2} M_{1}^{\prime} M_{2}^{\prime} \mid J_{1} J_{2} J M\right) \\
& =\delta_{M_{1} M_{1}^{\prime}} \delta_{M_{2} M_{2}^{\prime}} .
\end{aligned}
$$

The product of $\left|J_{1} M_{1}\right\rangle$ and $\left|J_{2} M_{2}\right\rangle$ will, under a rotation about the $z$-axis through $\phi$, be multiplied by $e^{i M_{1} \phi} e^{i M_{2} \phi}=e^{i\left(M_{1}+M_{2}\right) \phi}$ and hence $M=$ $M_{1}+M_{2}$, and all coefficients not meeting this requirement are zero. The index $M$ is frequently suppressed when written in the form $C_{M_{1}^{1} M_{2}{ }^{J}}^{J^{J}}$. Because of this relation between the $M_{1}$ and $M_{2}$, the sum in (5.5a) is in effect a sum over $M_{1}$ only (or $M_{2}$ only).

A general formula for these coefficients has been given by Wigner, who carried out in detail the process outlined above of reducing the direct product matrix. Condon and Shortley have approached the problem from the standpoint of transforming eigenstates of two commuting angular momenta $\mathbb{J}_{1}$ and $J_{2}$ with quantum numbers $J_{1} M_{1}$ and $J_{2} M_{2}$ into eigenstates of $\mathbb{J}=\mathbb{J}_{1}+\mathbb{J}_{2}$ with quantum numbers $J$ and $M$. They pointed out that the vector addition coefficients may in principle be obtained from the initial condition $\left(J_{1} J_{2} J_{1} J_{2}\left(J_{1} J_{2}\left[J_{1}+J_{2}\right]\left[J_{1}+J_{2}\right]\right)=1\right.$ and from a successive application of the $J_{x}-i J_{y}$ and $J_{1 z}$ operators, but that a general formula is difficult to obtain. Racah (1942a) utilizing as well the operator $J_{x}+i J_{y}$ has provided a purely algebraic derivation of a general formula for the coefficients which is relatively convenient and symmetric in the various parameters. Based upon this for- 
mula, Simon (1954) has provided a numerical table of these coefficients, but no $J$ greater than $1 \% 2$ is included. Condon and Shortley have given formulas for the cases where $J_{2}=1 / 2,1,3 / 2$, and 2 , while Falkoft et al. (1952) give $J_{2}=3$.

For actual computations, a numerical table of Wigner coefficients is required. The only such table known to the writer is that of Simon (1954). Unfortunately, this covers an inadequate range of values for rare-earth spectra, and relies on symmetry properties of the Wigner coefficients, involving factors of the form $\left(\frac{2 J+1}{2 J_{1}+1}\right)^{1 / 2}$, which are usually somewhat awkward. To meet partially this need for the present application, table 15, containing the coefficients $C_{M \mu \nu}^{\mathcal{S}_{\nu} \boldsymbol{y}}$ to 6 decimals for $J \leq 8, \nu=2,4,6$ and $\mu=0,2,3,4,6$ consistent with the $\nu$ involved has been computed. These will permit calculation of matrix elements diagonal in $J$ and between states of the same parity.

In the case $\mu=0,3$, and 6 , the values for $M=J-\mu$ were calculated from the formula of Racah and those for other values of $M$ were calculated by the use of the tables of Stevens and others noted abore (section 5.1). For $\mu=4$ and $\nu=4, \mu=2$, factor's colresponding to those of Stevens were computed and used in a similar process. For $\mu=2, \nu=6$ this became too unwieldly and the formula of Racah was used directly for all entries. Coefficients were all checked for obedience to the requirement

$$
\sum_{M I}\left(C_{M \mu}^{J J}\right)^{2}=\frac{2 J+1}{2 \nu+1}
$$

and selected entries were checked for

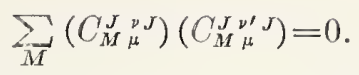

It is believed that errors do not exceed 2 in the sixth decimal. In a very few entries a seventh decinial was carried.

It is to be noted that only positive values of $\mu$ are given, and only entries for $M \geq-\frac{\mu}{2}$. To obtain other values required, the following special cases of the general symmetry relations are required (diagonal in $J$ and $\nu$ even):

$$
\begin{aligned}
C_{-M-\mu}^{J} \nu_{\mu}^{J} & =C_{M \mu}^{J \nu J} \\
C_{(M+\mu)-\mu}^{J}{ }^{\nu} J & =(-1)^{\mu} C_{M \mu}^{J \nu J} \\
C_{-\left(M^{\nu}+\mu\right) \mu}^{J J} & =(-1)^{\mu} C_{M \mu}^{J \nu J} .
\end{aligned}
$$

The relation (5.6c) is especially noteworthv: we observe that (for half-integral $\bar{J}$ )

$$
C_{-\frac{3}{2} 3 \frac{3}{2}}^{J \nu J}=(-1)^{3} C_{-\frac{3}{2} 3 \frac{3}{2}}^{J \nu J}
$$

\begin{tabular}{|c|c|c|c|c|c|c|c|c|c|}
\hline \multicolumn{10}{|c|}{$C_{M 20}^{J 2 S}$} \\
\hline & 0 & 1 & 2 & 3 & 4 & 5 & 6 & 7 & 8 \\
\hline $\begin{array}{l}1 \\
2 \\
3\end{array}$ & $\begin{array}{r}-0.632456 \\
-.534522 \\
-.516398\end{array}$ & $\begin{array}{r}0.316228 \\
-.267261 \\
-.387298\end{array}$ & $\begin{array}{c}\text { 0. } 534522 \\
0\end{array}$ & 0. 645497 & & & & & \\
\hline $\begin{array}{l}4 \\
5 \\
6\end{array}$ & $\begin{array}{l}-.509647 \\
-.506369 \\
-.504525\end{array}$ & $\begin{array}{l}-.433200 \\
-.455732 \\
-.468488\end{array}$ & $\begin{array}{l}-.203859 \\
-.303822 \\
-.360375\end{array}$ & $\begin{array}{r}.178376 \\
-.050637 \\
-.180188\end{array}$ & $\begin{array}{r}0.713506 \\
.303822 \\
.072075\end{array}$ & $\begin{array}{r}0.759554 \\
.396412\end{array}$ & 0. 792825 & & \\
\hline $\begin{array}{l}7 \\
8\end{array}$ & $\begin{array}{l}-.503382 \\
-.502625\end{array}$ & $\begin{array}{l}-.476415 \\
-.481682\end{array}$ & $\begin{array}{l}-.395515 \\
-.418854\end{array}$ & $\begin{array}{l}-.260680 \\
-.314140\end{array}$ & $\begin{array}{l}-.071912 \\
-.167542\end{array}$ & $\begin{array}{r}170790 \\
.020943\end{array}$ & $\begin{array}{r}467426 \\
251312\end{array}$ & $\begin{array}{r}0.817996 \\
.523568\end{array}$ & 0. 837708 \\
\hline$M$ & $1 / 2$ & $3 / 2$ & $5 / 2$ & $7 / 2$ & $9 / 2$ & $11 / 2$ & $13 / 2$ & $15 / 2$ & \\
\hline $\begin{array}{l}3 / 2 \\
5 / 2 \\
7 / 2\end{array}$ & $\begin{array}{r}-0.447214 \\
-.478091 \\
-.487950\end{array}$ & $\begin{array}{r}0.447214 \\
-.119523 \\
-.292770\end{array}$ & $\begin{array}{r}0.597614 \\
.097590\end{array}$ & 0. 683130 & & & & & \\
\hline $\begin{array}{r}9 / 2 \\
11 / 2 \\
13 / 2\end{array}$ & $\begin{array}{l}-.492366 \\
-.494728 \\
-.496139\end{array}$ & $\begin{array}{l}-.369274 \\
\text {-. } 409917 \\
-.434122\end{array}$ & $\begin{array}{l}-.123092 \\
-.240296 \\
-.310087\end{array}$ & $\begin{array}{r}.246183 \\
.014135 \\
-.124035\end{array}$ & $\begin{array}{r}0.738549 \\
.353377 \\
.124035\end{array}$ & $\begin{array}{r}0.777429 \\
.434122\end{array}$ & 0. 806228 & & \\
\hline $15 / 2$ & -.497050 & -.449712 & -.355036 & -.213022 & -.023669 & .213022 & .497050 & 0.828417 & \\
\hline
\end{tabular}

and hence must be zero. 'This is intimately associated with the essential degeneracy of Kramers discussed earlier.

TABLE 15. Wigner- coefficients 
TABLE 15. Wigner coefficients-Continued

$C_{M T 0}^{J 4 J}$

\begin{tabular}{|c|c|c|c|c|c|c|c|c|c|}
\hline & 0 & 1 & 2 & 3 & 4 & 5 & 6 & 7 & 8 \\
\hline $\begin{array}{l}2 \\
3 \\
4\end{array}$ & $\begin{array}{r}0.534523 \\
.426402 \\
.402291\end{array}$ & $\begin{array}{r}-0.356348 \\
.071067 \\
.201146\end{array}$ & $\begin{array}{r}0.089087_{1} \\
-.497469 \\
-.245844\end{array}$ & $\begin{array}{r}0.213201 \\
-.469340\end{array}$ & 0.312893 & & & & \\
\hline $\begin{array}{l}5 \\
6 \\
7\end{array}$ & $\begin{array}{r}392232 \\
.386953 \\
.383807\end{array}$ & $\begin{array}{r}261488 \\
.294821 \\
.315270\end{array}$ & $\begin{array}{r}-.065372 \\
.050672 \\
.127428\end{array}$ & $\begin{array}{l}\text {-. } 392232 \\
\text {-. } 248756 \\
-.126413\end{array}$ & $\begin{array}{l}-.392232 \\
-.442232 \\
-.357408\end{array}$ & $\begin{array}{r}0.392232 \\
-.304035 \\
-.441175\end{array}$ & $\begin{array}{r}0.456052 \\
-.217795\end{array}$ & 0. 508189 & \\
\hline 8 & .381771 & .328747 & 180281 & -.031814 & -.254514 & -.413585 & -.413585 & -.137862 & 0.551447 \\
\hline & $1 / 2$ & $3 / 2$ & $5 / 2$ & $7 / 2$ & $9 / 2$ & $11 / 2$ & $13 / 2$ & $15 / 2$ & \\
\hline $\begin{array}{l}5 / 2 \\
7 / 2 \\
9 / 2\end{array}$ & $\begin{array}{r}0.308606 \\
.341882 \\
.354787\end{array}$ & $\begin{array}{r}-0.462909 \\
-.113961 \\
.059131\end{array}$ & $\begin{array}{r}0.154303 \\
-.493829 \\
-.335077\end{array}$ & $\begin{array}{r}0.265908 \\
-.433629\end{array}$ & 0.354787 & & & & \\
\hline $\begin{array}{l}11 / 2 \\
13 / 2 \\
15 / 2\end{array}$ & $\begin{array}{l}.361298 \\
.365073 \\
.367467\end{array}$ & $\begin{array}{l}154842 \\
.212959 \\
.250811\end{array}$ & $\begin{array}{r}-.167745 \\
-.043944 \\
.044718\end{array}$ & $\begin{array}{l}-.425815 \\
-.310988 \\
-.196371\end{array}$ & $\begin{array}{r}-.348394 \\
-.446201 \\
-.390798\end{array}$ & $\begin{array}{r}0.425815 \\
-.260284 \\
-.429684\end{array}$ & $\begin{array}{r}0.483384 \\
-.176929\end{array}$ & 0.530786 & \\
\hline
\end{tabular}

$C_{M 0}^{J_{6} S}$

\begin{tabular}{|c|c|c|c|c|c|c|c|c|c|}
\hline$M$ & 0 & 1 & 2 & 3 & 4 & 5 & 6 & 7 & 8 \\
\hline $\begin{array}{l}3 \\
4 \\
5\end{array}$ & $\begin{array}{r}-0.482804 \\
-.373979 \\
-.347368\end{array}$ & $\begin{array}{r}0.362103 \\
.018599 \\
-.104210\end{array}$ & $\begin{array}{r}-0.144841 \\
.411377 \\
.312631\end{array}$ & $\begin{array}{r}0.024140_{2} \\
-.317882 \\
.251842\end{array}$ & $\begin{array}{c}0.074795_{8} \\
-.416842\end{array}$ & 0.130263 & & & \\
\hline $\begin{array}{l}6 \\
7 \\
8\end{array}$ & $\begin{array}{r}-.335531 \\
-.329015 \\
-.324985\end{array}$ & $\begin{array}{r}-.167765 \\
-.205635 \\
-.230198\end{array}$ & $\begin{array}{l}.184542 \\
.082254 \\
.005416\end{array}$ & $\begin{array}{r}.360696 \\
.324080 \\
.251864\end{array}$ & $\begin{array}{l}.067106 \\
.289534 \\
.346651\end{array}$ & $\begin{array}{r}-.461355 \\
-.090479 \\
.176034\end{array}$ & $\begin{array}{r}0.184542 \\
-.470492 \\
-.211240\end{array}$ & $\begin{array}{r}0.235246 \\
-.457688\end{array}$ & 0. 281654 \\
\hline & $1 / 2$ & $3 / 2$ & $5 / 2$ & $7 / 2$ & $9 / 2$ & $11 / 2$ & $13 / 2$ & $15 / 2$ & \\
\hline $\begin{array}{r}7 / 2 \\
9 / 2 \\
11 / 2\end{array}$ & $\begin{array}{r}-0.241402 \\
-.273115 \\
-.286829\end{array}$ & $\begin{array}{r}0.434524 \\
.204836 \\
.057366\end{array}$ & $\begin{array}{r}-0.241402 \\
.341393 \\
.358536\end{array}$ & $\begin{array}{r}0.048280_{5} \\
-.375533 \\
.157756\end{array}$ & $\begin{array}{r}0.102418 \\
-.444585\end{array}$ & 0.157756 & & & \\
\hline $\begin{array}{l}13 / 2 \\
15 / 2\end{array}$ & $\begin{array}{r}-.294280 \\
-.298843\end{array}$ & $\begin{array}{r}.036785 \\
-.099614\end{array}$ & $\begin{array}{l}.272209 \\
.179306\end{array}$ & $\begin{array}{l}.334007 \\
.346658\end{array}$ & $\begin{array}{r}-.016185 \\
.235090\end{array}$ & $\begin{array}{r}-.469376 \\
-.155398\end{array}$ & $\begin{array}{r}0.210410 \\
-.466195\end{array}$ & 0. 258997 & \\
\hline
\end{tabular}


TABLE 15. Wigner coefficients-Continued

\begin{tabular}{|c|c|c|c|c|c|c|c|c|}
\hline.$M$ & -1 & 0 & 1 & 2 & 3 & 4 & 5 & 6 \\
\hline $\begin{array}{l}1 \\
2 \\
3\end{array}$ & $\begin{array}{r}0.774597 \\
.654653 \\
.632455\end{array}$ & $\begin{array}{r}0.534522 \\
.577350\end{array}$ & 0.408248 & & & & & \\
\hline $\begin{array}{l}4 \\
5 \\
6\end{array}$ & $\begin{array}{r}.624188 \\
.620173 \\
.617915\end{array}$ & $\begin{array}{r}592156 \\
.599144 \\
.603023\end{array}$ & $\begin{array}{r}.495434 \\
.535891 \\
.558291\end{array}$ & $\begin{array}{r}\text { 0. } 330289 \\
.429669 \\
.483494\end{array}$ & $\begin{array}{r}0.277350 \\
.377965\end{array}$ & 0.239046 & & \\
\hline $\begin{array}{l}7 \\
8\end{array}$ & 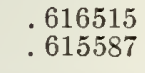 & $\begin{array}{r}605405 \\
.606977\end{array}$ & $\begin{array}{r}572054 \\
.581136\end{array}$ & $\begin{array}{r}.516377 \\
.538028\end{array}$ & $\begin{array}{l}.438160 \\
.477567\end{array}$ & $\begin{array}{r}336817 \\
.399561\end{array}$ & $\begin{array}{r}0.210042 \\
.303489\end{array}$ & 0.187317 \\
\hline$M$ & $-1 / 2$ & $1 / 2$ & $3 / 2$ & $5 / 2$ & $7 / 2$ & $9 / 2$ & $11 / 2$ & \\
\hline $\begin{array}{l}3 / 2 \\
5 / 2 \\
7 / 2\end{array}$ & $\begin{array}{r}0.632456 \\
.621059 \\
.617213\end{array}$ & $\begin{array}{r}0.462910 \\
.534522\end{array}$ & 0. 365148 & & & & & \\
\hline $\begin{array}{r}9 / 2 \\
11 / 2 \\
13 / 2\end{array}$ & $\begin{array}{r}.615457 \\
.614509 \\
.613941\end{array}$ & $\begin{array}{r}564075 \\
.579365 \\
.588349\end{array}$ & $\begin{array}{r}.460566 \\
.508862 \\
.537087\end{array}$ & $\begin{array}{r}0.301511 \\
.402291 \\
.459933\end{array}$ & $\begin{array}{r}0.256776 \\
.356263\end{array}$ & 0. 223607 & & \\
\hline $15 / 2$ & . 613572 & .594088 & .555088 & . 496486 & . 418079 & . 319313 & 0. 198030 & \\
\hline
\end{tabular}

$C_{M 2}^{J 4 J}$

\begin{tabular}{|c|c|c|c|c|c|c|c|c|}
\hline 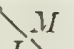 & -1 & 0 & 1 & 2 & 3 & 4 & 5 & 6 \\
\hline $\begin{array}{l}2 \\
3 \\
4\end{array}$ & $\begin{array}{r}-0.563436 \\
-.449467 \\
-.424052\end{array}$ & $\begin{array}{r}0.345033 \\
-.123091 \\
-.245844\end{array}$ & $\begin{array}{r}0.522233 \\
.186989\end{array}$ & 0.560968 & & & & \\
\hline $\begin{array}{l}5 \\
6 \\
7\end{array}$ & $\begin{array}{r}-.413449 \\
-.407884 \\
-.404568\end{array}$ & $\begin{array}{l}-.299572 \\
-.328395 \\
-.345779\end{array}$ & $\begin{array}{l}0 \\
-.110558 \\
-.180745\end{array}$ & $\begin{array}{r}.358057 \\
.183513 \\
.056476\end{array}$ & $\begin{array}{r}0.554700 \\
.449089 \\
.308827\end{array}$ & $\begin{array}{r}0.532554 \\
.495260\end{array}$ & 0. 505389 & \\
\hline 8 & -.402422 & -.357114 & -.227940 & -.035172 & .187317 & 391802 & .515831 & 0.477567 \\
\hline$M$ & $-1 / 2$ & $1 / 2$ & $3 / 2$ & $5 / 2$ & $7 / 2$ & $9 / 2$ & $11 / 2$ & \\
\hline $\begin{array}{l}5 / 2 \\
7 / 2 \\
9 / 2\end{array}$ & $\begin{array}{r}-0.345033 \\
-.372194 \\
-.381690\end{array}$ & $\begin{array}{r}0.462910 \\
-.053722 \\
-.116608\end{array}$ & $\begin{array}{r}0.550482 \\
.285631\end{array}$ & 0.560968 & & & & \\
\hline $\begin{array}{l}11 / 2 \\
13 / 2 \\
15 / 2\end{array}$ & $\begin{array}{l}-.386244 \\
-.388809 \\
-.390406\end{array}$ & $\begin{array}{l}-.204837 \\
-.256681 \\
-.289807\end{array}$ & $\begin{array}{r}.099950 \\
-.022676 \\
-.105958\end{array}$ & $\begin{array}{l}.410891 \\
.252439 \\
.126363\end{array}$ & $\begin{array}{r}\text { 0. } 544705 \\
.476312 \\
.354689\end{array}$ & $\begin{array}{r}0.519238 \\
.507935\end{array}$ & . 491412 & \\
\hline
\end{tabular}


TABLE 15. Wigner coefficients-Continued

$C_{M 2}^{J 6 J}$

\begin{tabular}{|c|c|c|c|c|c|c|c|c|}
\hline$M$ & -1 & 0 & 1 & 2 & 3 & 4 & 5 & 6 \\
\hline $\begin{array}{l}3 \\
4 \\
5\end{array}$ & $\begin{array}{r}0.494727 \\
.383214 \\
.355945\end{array}$ & $\begin{array}{r}-0.361298 \\
\quad 0 \\
\quad .122813\end{array}$ & $\begin{array}{r}0.127738 \\
-.434524 \\
-.302079\end{array}$ & $\begin{array}{r}0.289683 \\
-.308257\end{array}$ & 0.397959 & & & \\
\hline $\begin{array}{l}6 \\
7 \\
8 \\
\end{array}$ & $\begin{array}{r}.343817 \\
.337140 \\
.333010 \\
\end{array}$ & $\begin{array}{l}184542 \\
220710 \\
243919 \\
\end{array}$ & $\begin{array}{r}-.163087 \\
-.058394 \\
.017964 \\
\end{array}$ & $\begin{array}{r}-.376632 \\
-.316265 \\
-.232843 \\
\end{array}$ & $\begin{array}{l}-.147214 \\
-.335450 \\
-.361685 \\
\end{array}$ & $\begin{array}{l}0.465530 \\
0 \\
-.247027 \\
\end{array}$ & $\begin{array}{r}0.505389 \\
.121960 \\
\end{array}$ & 0.526926 \\
\hline$M$ & $-1 / 2$ & $1 / 2$ & $3 / 2$ & $5 / 2$ & $7 / 2$ & $9 / 2$ & $11 / 2$ & \\
\hline $\begin{array}{r}7 / 2 \\
9 / 2 \\
11 / 2\end{array}$ & $\begin{array}{r}255476 \\
.285631 \\
.298081\end{array}$ & $\begin{array}{r}-.442498 \\
-.186989 \\
-.035129\end{array}$ & $\begin{array}{r}.215917 \\
-.381691 \\
-.359967\end{array}$ & $\begin{array}{r}.349825 \\
-.227663\end{array}$ & 435942 & & & \\
\hline $\begin{array}{l}13 / 2 \\
15 / 2\end{array}$ & $\begin{array}{r}304672 \\
.308643\end{array}$ & $\begin{array}{r}058394 \\
.119537\end{array}$ & $\begin{array}{r}-.255871 \\
-.156366\end{array}$ & $\begin{array}{l}-.365192 \\
-.349645\end{array}$ & $\begin{array}{l}-.070719 \\
-.294427\end{array}$ & $\begin{array}{r}488252 \\
.064249\end{array}$ & .517994 & \\
\hline
\end{tabular}

\section{$C_{M 3}^{J 4 J}$}

\begin{tabular}{|c|c|c|c|c|c|c|c|}
\hline$M$ & -1 & 0 & 1 & 2 & 3 & 4 & 5 \\
\hline $\begin{array}{l}2 \\
3 \\
4\end{array}$ & $\begin{array}{r}-0.527046 \\
-.265908 \\
-.186989\end{array}$ & $\begin{array}{r}-0.564076 \\
-.469339\end{array}$ & -0.494727 & & & & \\
\hline 5 & -.146176 & -.392232 & -.506369 & -0.423659 & & & \\
\hline 6 & -.120654 & -.335111 & -.472034 & -.490098 & -0.363803 & & \\
\hline & & & & & & & \\
\hline 8 & -.089984 & -.258460 & -.393234 & -.474495 & -.486664 & -.420595 & -0.275723 \\
\hline & $-1 / 2$ & $1 / 2$ & $3 / 2$ & $5 / 2$ & $7 / 2$ & $9 / 2$ & \\
\hline $5 / 2$ & -0.577350 & & & & & & \\
\hline $\begin{array}{l}7 / 2 \\
9 / 2\end{array}$ & $\begin{array}{r}-.402015 \\
-.311649\end{array}$ & $\begin{array}{r}-0.531816 \\
-.498639\end{array}$ & -0.458029 & & & & \\
\hline $11 / 2$ & -.255476 & -.442497 & -.501745 & -0.392232 & & & \\
\hline $13 / 2$ & -.216867 & -.391883 & -.487398 & -.474594 & -0.338200 & & \\
\hline $15 / 2$ & -.188584 & -.349644 & -.457792 & -.492112 & -. 438906 & -0.294427 & \\
\hline
\end{tabular}

$C_{M 3}^{J_{6} J}$

\begin{tabular}{|c|c|c|c|c|c|c|c|}
\hline$M$ & -1 & 0 & 1 & 2 & 3 & 4 & 5 \\
\hline $\begin{array}{l}3 \\
4 \\
5\end{array}$ & $\begin{array}{r}\text { 0. } 469340 \\
.270973 \\
.201802\end{array}$ & $\begin{array}{r}-0.221249 \\
.323875 \\
.376036\end{array}$ & $\begin{array}{r}-0.409673 \\
.058255\end{array}$ & -0.487398 & & & \\
\hline $\begin{array}{l}6 \\
7 \\
8\end{array}$ & $\begin{array}{r}163087 \\
.137637 \\
.119407\end{array}$ & $\begin{array}{r}360696 \\
.332940 \\
.304863\end{array}$ & $\begin{array}{l}248129 \\
.322787 \\
.347875\end{array}$ & $\begin{array}{r}-.147214 \\
.079066 \\
.209882\end{array}$ & $\begin{array}{r}-0.509963 \\
-.258078 \\
-.071755\end{array}$ & $\begin{array}{r}-0.505389 \\
-.372080\end{array}$ & -0.487838 \\
\hline
\end{tabular}


TABLE 15. Wigner coefficients-Continued

\begin{tabular}{|c|c|c|c|c|c|c|}
\hline$M$ & $-1 / 2$ & $1 / 2$ & $3 / 2$ & $5 / 2$ & $7 / 2$ & $9 / 2$ \\
\hline $\begin{array}{r}7 / 2 \\
9 / 2 \\
11 / 2\end{array}$ & $\begin{array}{r}0.442498 \\
.373979 \\
.316163\end{array}$ & $\begin{array}{r}-0.334497 \\
.186990 \\
.324509\end{array}$ & $\begin{array}{r}-0.458029 \\
-.053661\end{array}$ & -0.503382 & & \\
\hline $\begin{array}{l}13 / 2 \\
15 / 2\end{array}$ & $\begin{array}{r}272507 \\
.239074\end{array}$ & $\begin{array}{r}357008 \\
.354605\end{array}$ & $\begin{array}{r}163319 \\
.270834\end{array}$ & $\overline{0}_{0} .223634$ & $\begin{array}{r}-0.509963 \\
-.333849\end{array}$ & -0.497673 \\
\hline
\end{tabular}

$C_{M 4}^{J U J}$

\begin{tabular}{|c|c|c|c|c|c|c|c|}
\hline$M$ & -2 & -1 & 0 & 1 & 2 & 3 & 4 \\
\hline $\begin{array}{l}2 \\
3 \\
4\end{array}$ & $\begin{array}{r}0.745356 \\
.594588 \\
.560968\end{array}$ & $\begin{array}{r}0.460566 \\
.494727\end{array}$ & 0.312893 & & & & \\
\hline $\begin{array}{l}5 \\
6 \\
7\end{array}$ & $\begin{array}{l}.546941 \\
.539583 \\
.535193\end{array}$ & $\begin{array}{r}.506369 \\
.511894 \\
.514990\end{array}$ & $\begin{array}{r}392232 \\
.432629 \\
.456490\end{array}$ & $\begin{array}{r}0.226455 \\
.313114 \\
.366006\end{array}$ & $\begin{array}{r}0.171499 \\
.253968\end{array}$ & 0.134387 & \\
\hline 8 & .532354 & .516920 & .471881 & .401022 & .310630 & 209427 & 0.108148 \\
\hline$M$ & $-3 / 2$ & $-1 / 2$ & $1 / 2$ & $3 / 2$ & $5 / 2$ & $7 / 2$ & \\
\hline $\begin{array}{l}5 / 2 \\
7 / 2 \\
9 / 2\end{array}$ & $\begin{array}{r}0.577350 \\
.550482 \\
.539792\end{array}$ & $\begin{array}{r}0.376051 \\
.440738\end{array}$ & 0. 264443 & & & & \\
\hline $\begin{array}{l}11 / 2 \\
13 / 2 \\
15 / 2\end{array}$ & $\begin{array}{l}.534366 \\
.531214 \\
.529213\end{array}$ & $\begin{array}{r}469339 \\
.484930 \\
.494472\end{array}$ & $\begin{array}{r}.349825 \\
.397958 \\
.428225\end{array}$ & $\begin{array}{r}0.196116 \\
.281399 \\
.336926\end{array}$ & $\begin{array}{c}0.151247_{5} \\
.230164\end{array}$ & 0. 120199 & \\
\hline
\end{tabular}

\section{$C_{M 4}^{J 6 J}$}

\begin{tabular}{|c|c|c|c|c|c|c|c|}
\hline$M$ & -2 & -1 & 0 & 1 & 2 & 3 & 4 \\
\hline $\begin{array}{l}3 \\
4 \\
5\end{array}$ & $\begin{array}{r}-0.541947 \\
-.419790 \\
-.389919\end{array}$ & $\begin{array}{r}0.349825 \\
-.079333 \\
-.195539\end{array}$ & $\begin{array}{r}0.501745 \\
.233021\end{array}$ & 0.504505 & & & \\
\hline $\begin{array}{l}6 \\
7 \\
8\end{array}$ & $\begin{array}{r}-.376632 \\
-.369318 \\
-.364795\end{array}$ & $\begin{array}{l}\text {-. } 248128 \\
\text {-. } 277194 \\
-.295182\end{array}$ & $\begin{array}{r}.067106 \\
-.037801 \\
-.0707785\end{array}$ & $\begin{array}{r}382472 \\
247517 \\
137400\end{array}$ & $\begin{array}{r}0.465530 \\
.441640 \\
.354765\end{array}$ & $\begin{array}{r}0.417311 \\
.454447\end{array}$ & 0. 370540 \\
\hline$M$ & $-3 / 2$ & $-1 / 2$ & $1 / 2$ & $3 / 2$ & $5 / 2$ & $7 / 2$ & \\
\hline $\begin{array}{r}7 / 2 \\
9 / 2 \\
11 / 2\end{array}$ & $\begin{array}{r}-0.312893 \\
-.334497 \\
-.341495\end{array}$ & $\begin{array}{r}0.458029 \\
.102418 \\
-.055544\end{array}$ & $\begin{array}{r}0.512092 \\
.322921\end{array}$ & 0. 487398 & & & \\
\hline $\begin{array}{l}13 / 2 \\
15 / 2\end{array}$ & $\begin{array}{r}-.344698 \\
-.346452\end{array}$ & $\begin{array}{l}-.141599 \\
-.194225\end{array}$ & $\begin{array}{r}167849 \\
.056068\end{array}$ & $\begin{array}{l}419971 \\
308798\end{array}$ & $\begin{array}{r}\text { 0. } 441641 \\
.452034\end{array}$ & 0.393445 & \\
\hline
\end{tabular}


TABLE 15. Wigner coefficients-Continued

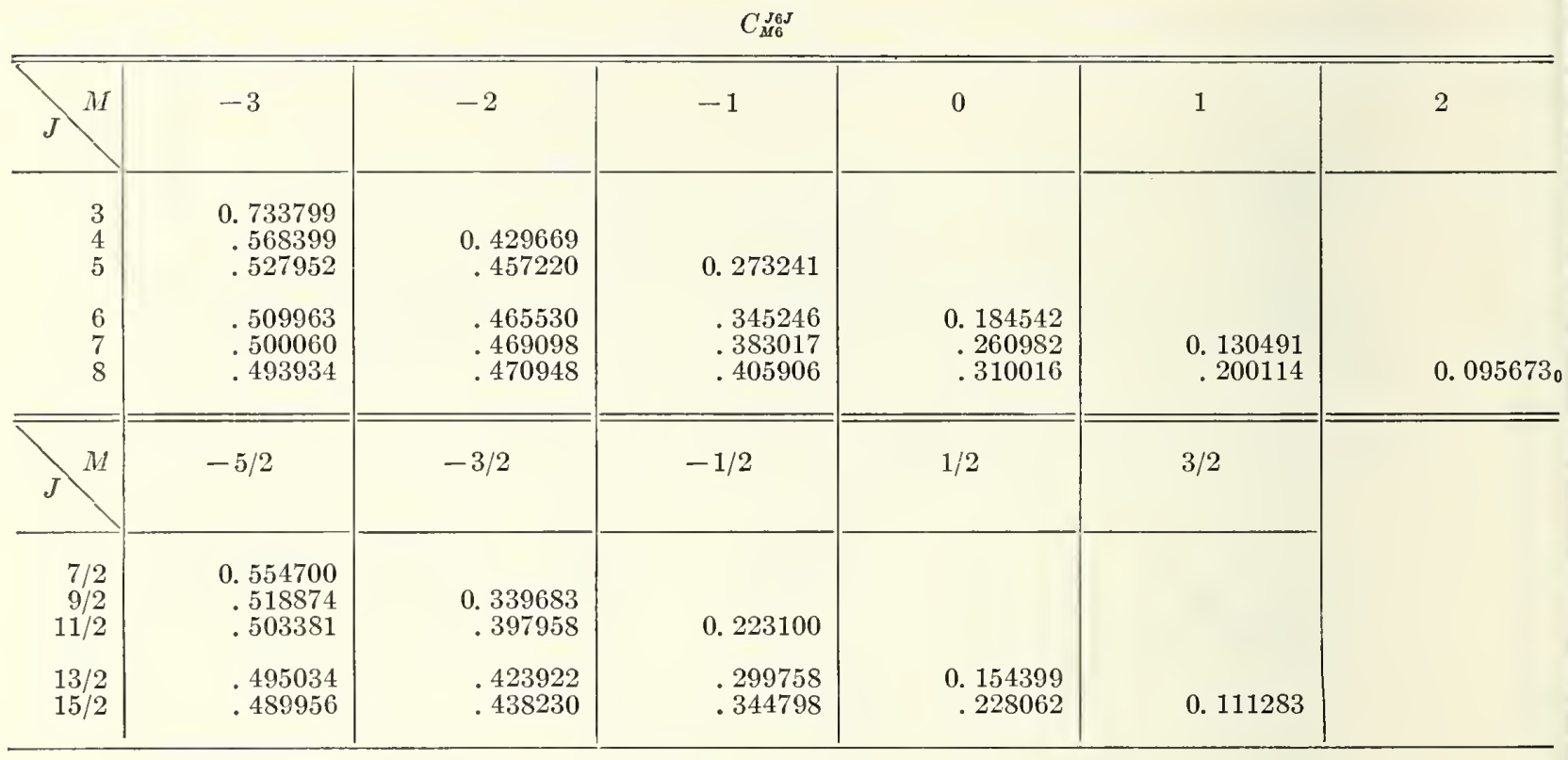

\section{3. $L-S$ Coupling and the One-Electron Approximation}

It is well to emphasize at this point that the relation (5.4) involves no approximations - these we shall introduce in evaluating the reduced matrix element. The first of these is the approximation of $L-S$ coupling, i.e., the state $|\alpha J M\rangle$ may be written $\left.\left|\alpha J M>=\sum_{M_{L}}\right| \beta L S M_{L} M_{S}\right\rangle$ $\left(L S M_{L} M_{S} \mid L S J M\right)$, and consequently,

$\left\langle\alpha J^{\prime} M^{\prime}\left|Y_{\nu}^{\mu}\right| \alpha J M\right\rangle=\sum_{M_{L}^{\prime} M_{L}}\left(L^{\prime} S^{\prime} J^{\prime} M^{\prime} \mid L^{\prime} S^{\prime} M_{L}^{\prime} M_{S}^{\prime}\right)$ $\left.<\beta^{\prime} L^{\prime} S^{\prime} M_{L}^{\prime} M_{S}^{\prime}\left|Y_{\nu}^{\mu}\right| \beta L S M_{L} M_{S}\right\rangle\left(L S M_{L} M_{S} \mid L S J M\right)$.

We now recall that $Y_{\nu}^{\mu}$ does not act upon the spin coordinates of the system, that it is thus a scalar quantity with respect to $S$, and therefore

$\left\langle\beta^{\prime} L^{\prime} S^{\prime} M_{L}^{\prime} M_{S}^{\prime}\left|Y_{\nu}^{\mu}\right| \beta L S M_{L} M_{S}\right\rangle=\delta_{S S^{\prime}} \delta_{M_{S} M_{S}^{\prime}}$

$$
\left\langle\beta^{\prime} L^{\prime} S M_{L}^{\prime} M_{S}\left|Y_{\nu}^{\mu}\right| \beta L S M_{L} M_{S}\right\rangle \text {. }
$$

This will reduce (5.7) to the equation

$\left\langle\alpha^{\prime} J^{\prime} M^{\prime}\left|Y_{\nu}^{\mu}\right| \alpha J M\right\rangle=\delta_{S S^{\prime}} \sum_{M_{L}^{\prime} M_{L}}$

$\left(L^{\prime} S J^{\prime} M^{\prime} \mid L^{\prime} S M_{L}^{\prime} M_{S}\right)$

$\left.<\beta^{\prime} L^{\prime} S M_{L}^{\prime} M_{s}\left|Y_{\nu}^{\mu}\right| \beta L S M_{L} M_{S}\right\rangle\left(L S M_{L} M_{S} \mid L S J M\right)$.

All other matrix elements vanish-in particular we see that there are no matrix elements between terms of different multiplicities. Also we see that
(5.4) is again applicable, and obtain

$$
\begin{array}{r}
\left.<\beta^{\prime} L^{\prime} S M_{L}^{\prime} M_{S}\left|Y_{\nu}^{\mu}\right| \beta L S M_{L} M_{S}\right\rangle=\left(L v L^{\prime} M_{L}^{\prime} \mid L \nu M_{L} \mu\right) \\
\left(\beta^{\prime} L^{\prime} S\left\|Y_{\nu}\right\| \beta L S\right) .
\end{array}
$$

Hence, we can write (5.9) in the form

$$
\begin{gathered}
<\beta^{\prime} L^{\prime} S J^{\prime} M^{\prime}\left|Y_{\nu}^{\mu}\right| \beta L S J M>=\sum_{M_{L} M_{L}^{\prime}} \\
\left(L^{\prime} S J^{\prime} M^{\prime} \mid L^{\prime} S M_{L}^{\prime} M_{S}\right)\left(L \nu L^{\prime} M_{L}^{\prime} \mid L \nu M_{L} \mu\right) \\
\left(L S M_{L} M_{S} \mid L S J M\right)\left(\beta^{\prime} L^{\prime} S\left\|Y_{\nu}\right\| \beta L S\right),
\end{gathered}
$$

and this expression is also (5.4). If we multiply both expressions by $\left(J \nu J^{\prime} M^{\prime} \mid J \nu M \mu\right)$, introduce the requirement that $M_{L}^{\prime}=M_{L}+\mu$, and sum over $M$, the expression on the right of (5.4) will reduce to $\left(\alpha^{\prime} J^{\prime}\left\|\mathbf{Y}_{\nu}\right\| \alpha J\right)$ by (5.5a) while the right side of (5.11) becomes

$$
\begin{gathered}
\sum_{M, M_{L}}\left(J \nu J^{\prime} M^{\prime} \mid J \nu M \mu\right)\left(L^{\prime} S J^{\prime} M^{\prime} \mid L^{\prime} S\left(M_{L}+\mu\right) M_{S}\right) \\
\left(L \nu L^{\prime}\left[M_{L}+\mu\right] \mid L \nu M_{L} \mu\right) \\
\left(L S M_{L} M_{S} \mid L S J M\right)\left(\beta^{\prime} L^{\prime} S|| Y_{\nu} \| \beta L S\right) .
\end{gathered}
$$

Such a sum of the product of 4 Wigner coefficients appears recurringly in the theory of complex spectra. The properties of such expressions were first studied extensively by Racah (1942a), and they have consequently become known as Racah coefficients. In the present application the essential significance of (5.11) is that the two states $J M$ and $J^{\prime} M^{\prime}$ are "uncoupled" into $L S M_{L} M_{S}$ and $L^{\prime} S^{\prime} M_{L}^{\prime} M_{S}^{\prime}$ so that the effect of the $Y_{v}^{\prime}$ acting only on the $L$ part (i.e., the space coordinates) may be determined. The Racah coeffi- 
cients appear generally in connection with such uncoupling or recoupling of angular momenta. The Racah coefficient in (5.12) depends on six parameters $J J^{\prime}, L L^{\prime}, S$, and $\nu$; in spite of the explicit appearance of the projection quantum numbers $M, M^{\prime}, M_{L}, M_{S}$, and $\mu$, they are eliminated in the summation.

Although a general algebraic formula for these coefficients is quite unwieldy, numerical tables have been computed by Biedenharn (1952) and by Simon et al. (1954). The first of these carries the entries in a closed fractional form, the other has a ten-place decimal form. In order to clarify difference in notation, let us rewrite (5.12) as

$$
\begin{aligned}
\left(\alpha^{\prime} J^{\prime}\left\|Y_{\nu}\right\| \alpha J\right)= & \sum_{M, M_{L}} C_{M \mu}^{J \nu J^{\prime}} C_{\left(M_{L}+\mu\right) M_{S}}^{L^{\prime}}{ }^{S} J^{\prime} C_{M_{L^{\mu}}}^{L \nu^{\prime}} \\
& C_{M_{L} M_{S}}^{L}{ }^{S J}\left(\beta^{\prime} L^{\prime} S\left\|Y_{\nu}\right\| \beta L S\right) .
\end{aligned}
$$

Omitting algebraic details, the use of the symmetry relations for the IVigner coefficients will permit this to be transformed into

$$
\begin{aligned}
& (-1)^{L+S-J^{\prime}-\nu} \sqrt{\frac{(2 J+1)\left(2 L^{\prime}+1\right)}{(2 S+1)(2 \nu+1)}} \\
& \sum_{M, M_{L}} C_{M M_{L}}^{J L S} C_{\left(M+M_{L}\right)\left(M^{\prime}-M-M_{L}\right)}^{L^{\prime}} J^{\prime} \\
& C_{M_{L}\left(M^{\prime}-M-M_{L}\right)}^{L}{ }^{\nu} C_{M\left(M K^{\prime}-M\right)}^{J} \stackrel{{ }^{\prime}}{{ }^{\prime}{ }^{\prime}} \cdot\left(\beta^{\prime} L^{\prime} S\left\|Y_{\nu}\right\| \beta L S\right)
\end{aligned}
$$

and a direct comparison with the notation of this with"eq (1), page $x$ of Simon et al. will give

$$
\begin{gathered}
\left(\alpha^{\prime} J^{\prime}\left\|Y_{\nu}\right\| \alpha J\right)=(-1)^{L+S-J^{\prime}-\nu} \sqrt{(2 J+1)\left(2 L^{\prime}+1\right)} \\
W^{\top}\left(J L J^{\prime} L^{\prime} ; S \nu\right)\left(\beta^{\prime} L^{\prime} S\left\|Y_{\nu}\right\| \beta L S\right)
\end{gathered}
$$

The six-parameter expression $W$ is tabulated in Simon et al. for $J, J^{\prime} \leq 15 / 2 ; L, L^{\prime} \leq 9 / 2$ (though of course a half-integral $L$ has no significance in the present application) $S \leq 3$ and $\nu \leq 8$, though the useful range of values may be extended through the use of the symmetry relations given in the introduction to the tables.

We are now in a position to make comparisons between quantities which depend only on the ratios of the matrix elements for a given $\beta^{\prime} L^{\prime} S$, $\beta L S$ combination, but for additional details we must evaluate the reduced matrix element $\left(\beta^{\prime} L^{\prime} S\left\|Y_{\nu}\right\| \beta L S\right)$. In principle, this maybe accomplished in a way similar to the derivation of (5.13) i.e., we now assume that the state $\mid L S M_{L} M_{S}>$ may be written as a sum of antisymmetrized products of one-electron wave functions $\left|n l m_{l} m_{s}\right\rangle$ and the matrix element (5.10) can be expressed in terms of one-electron matrix elements such as

$$
\left\langle n^{\prime} l^{\prime} m_{l}^{\prime} m_{s}^{\prime}\left|Y_{\nu}^{\mu}\right| n l m_{l} m_{s}\right\rangle \text {. }
$$

Unfortunately, this procedure has several limi- tations. First, there is the additional assumption that the state involves only one configuration of the free ion. In the present case, it is expected that configurations other than $4 f^{n}$ will interact with the surroundings and other rare earth ions to such an extent that they will no longer be characterized by sharp energy levels, but by broad levels or bands. The sharpness of the observed lines suggests that the contribution of other configurations to the wave functions of the free ion is, at least as far as the energy is concerned, negligible. It is quite possible that contributions from other configurations are responsible for features such as intensities and polarization; this will be discussed further in the following section.

An additional disadvantage is that, unlike the transformation from a $J M$ representation to an $L S$ representation, there is no general formula for passage from the $L S M_{L} M_{S}$ representation of a state to its one-electron representation. The general method of Gray and Wills (Condon and Shortley $5^{8}$ ) may be used, but each configuration must be considered by itself, and no general formula can be derived.

\subsection{The Rare Earth Ground Terms}

In the evaluation of the matrix element (5.10) we are free to select any consistent set of the projection quantum numbers; we shall therefore consider the specific element $\left[M_{L}^{\prime}=L^{\prime} ; M_{S}=S\right]$

$$
\begin{aligned}
\left\langle\beta^{\prime} L^{\prime} S L^{\prime} S\left|Y_{\nu}^{\mu}\right| \beta L S L S\right\rangle= & \left(L \nu L^{\prime} L^{\prime} \mid L_{\nu} L \mu\right) \\
& \left(\beta^{\prime} L^{\prime} S|| Y_{\nu} \mid \beta L S\right) .
\end{aligned}
$$

By selecting the maximum values of the projection quantum numbers, we shall find that the transformation to the one-electron representations is thereby simplified considerably.

For any particular rare earth, the analysis is best carried forward in a systematic manner, beginning with the ground state. In accordance with Hund's rules, for $4 f^{n}$ we select the highest allowed multiplicity, and then the highest $L$ consistent with this multiplicity. The corresponding antisymmetrized products of one-electron wave functions are usually written $\left\{\begin{array}{l}+- \\ m_{1} m_{1} m_{2}\end{array}\right\}$; for example, in this notation we start with $\left\{\begin{array}{l}+++ \\ 32\end{array}\right\}$ for

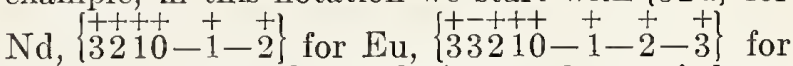
$\mathrm{Tb}$, etc. While the analysis may be carried out for any configuration, we shall consider the specific example of $\mathrm{Nd}$.

Let us begin with $\left\{\begin{array}{ll}+++ & 21\end{array}\right\}=\mid{ }^{4} I 6 \frac{3}{2}>$. The application of the symmetric operator $\mathscr{L}_{-}=L_{x}-i L_{y}$ (Condon and Shortley $5^{8} 3$ ) yields $\left\{\begin{array}{l}+++ \\ 320\end{array}\right\}=\mid{ }^{4} I 5 \frac{3}{2}>$. A second application of $\mathscr{L}$ yields $2 \sqrt{3}\left\{\begin{array}{l}++ \\ 32-1\end{array}\right\}$ $+\sqrt{10}\left\{\begin{array}{l}+++ \\ 310\end{array}\right\}=\left.\sqrt{22}\right|^{4} I 4 \frac{3}{2}>$. The state $\frac{1}{\sqrt{22}}[\sqrt{10}$ 
$\left.\left\{\begin{array}{l}+++ \\ 32\end{array}-\stackrel{+}{1}\right\}-2 \sqrt{3}\left\{\begin{array}{l}+++ \\ 3 \\ 10\end{array}\right\}\right]$ is orthogonal to $\mid{ }^{4} I 4 \frac{3}{2}>$ and is also a quartet with $M_{L}=4$ and hence is the state $\left|{ }^{4} G 4 \frac{3}{2}\right\rangle$. Repeated application of $\mathscr{L}_{-}$ will yield the states $\mid{ }^{4} F 3 \frac{3}{2}>,{ }^{4} \mathrm{D} 2 \frac{3}{2}>$, and $\mid{ }^{4} S 0 \frac{3}{2}>$, all of which arise from $4 f^{3}$ (Condon and Shortley able $1^{7}$ ). Similarly, beginning with $\left\{\begin{array}{c}+-+ \\ 332\end{array}\right\}=\mid{ }^{2} L 8 \frac{1}{2}>$ we can obtain the state $\mid{ }^{2} K 7 \frac{1}{2}>$. There are a total of four states with $M_{L}=6, M_{S}^{\prime}=\frac{1}{2}$, namely $\left|{ }^{2} L 6 \frac{1}{2}>,\right|{ }^{2} K 6 \frac{1}{2}>, \mid{ }^{2} I 6 \frac{1}{2}>$ and $\mid{ }^{4} I 6 \frac{1}{2}>$. The first two are obtained by a further application of $\mathscr{L}_{-}$ while the latter is obtained from $\mid{ }^{4} I 6 \frac{3}{2}>$ by the operator $\mathscr{S}_{-}=S_{x}-i S_{\nu}$. The fourth state is then $\left|{ }^{2} I 6 \frac{1}{2}\right\rangle$.

In endeavoring to continue this process to states where $M_{L}=5$, we find that in addition to those arising from higher values of $L$ or $S$, that there are two additional states with $M_{L}=5, M_{S}=\frac{1}{2}$. They must be therefore both described as ${ }^{2} H 5 \frac{1}{2}>$, and additional labels are necessary to distinguish them. The usual procedure is to explicitly diagonalize the submatrix of electrostatic interaction between the two states (Condon and Shortley $7^{8}$ ), but further work of Racah (1949) has shown that a more abstract classification of states based on group theory is possible. The former technique has been applied by Judd (1955) to the specific case of Eu.

In any perturbation calculation, the matrix elements first to be considered are those between different states of a degenerate level. In the rare earth ions, and in crystal spectra generally, the one most important level is the ground level. While the great significance of optical spectroscopy, in contrast to magnetic resonance measure- ments, lies in data provided concerning excited levels, a general ion-by-ion analysis of the excited levels is a major undertaking, and only the explicit details for the ground multiplet will be developed here. As a specific example, we shall further consider the case of $\mathrm{Nd}$, where (5.15) becomes

$$
\begin{aligned}
& <4 f^{3}{ }^{4} I 6 \frac{3}{2}\left|Y_{p}^{0}\right| 4 f^{3}{ }^{4} I 6 \frac{3}{2}>
\end{aligned}
$$

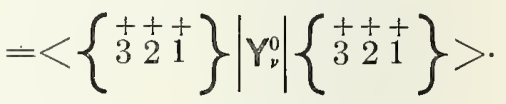

We shall here insert again the factor $r^{\nu}$ in the potential, omitted since (5.1) since it is invariant under the transformations we have been considering up to this point. Again recalling that $r^{\nu} Y_{\nu}^{0}$ is an electrostatic perturbation upon the individual electrons, we see that it is a spin-free quantity of Condon and Shortley type $F\left(6^{6} 9\right)$, and $(5.16)$ becomes $\sum_{m=1}^{3}\left\langle\left. 4 f \stackrel{+}{m}\right|^{\nu} \Theta_{\nu}^{0} \Phi^{0} \mid 4 f \stackrel{+}{m}\right\rangle$, where $\Phi^{0}=\frac{1}{\sqrt{2 \pi}}$ and $\theta_{\nu}^{0}$ is given in table 1. The spin terms of the matrix element give +1 , the $\phi$ terms give merely $\frac{1}{\sqrt{2 \pi}}$ and the $\theta$ integral is

$$
\int_{0}^{\pi} \theta_{3}^{m} \theta_{\nu}^{0} \theta_{3}^{m} \sin \theta d \theta=\sqrt{\frac{2 \nu+1}{2}} c^{\nu}(3 m, 3 m)
$$

where $c^{v}$ is given in table $1^{6}$ of Condon and Shortley. For the time being, we shall write the $r^{\nu}$ integral as $\left\langle r^{\nu}\right\rangle$.

By evaluating (5.14) and (5.15) for all the rare earths, the reduced matrix elements for the ground levels apart from the term $\left\langle r^{\nu}\right\rangle$ have been computed and presented in table 16. The reduced $L-S$ matrix elements are included principally for information, though they are directly useful in cases where the electrostatic perturbation is greater than the spin-orbit interaction, or where the electrostatic and spin-orbit perturbations must be considered simultaneously.

\section{Applications}

\subsection{General Considerations}

In this concluding section, we shall discuss further the general significance of the preceding sections in the interpretation of crystal spectra, the connection mentioned at the beginning of section 3.4 between the group-theory technique of sections 3 and 4 , and the matrix element calculations of section 5, and shall illustrate the application of these general techniques to specific problems.

Generally, a spectrum may be considered as understood if the spacing of the energy levels is known, the effect upon them of a magnetic field, selection and polarization rules and relative intensities of lines, and all these are correlated with the structure of the atom responsible for the spectrum. These may all in principle be obtained from a knowledge of the initial and final states of the transition involved in the spectral line. Unfortunately, our knowledge of these initial and final states is largely a matter of approximation which is not always entirely satisfactory. This is true both for the free ions and for the perturbed ions in the crystal. Let us review briefly the situation for the free ion.

The good quantum numbers for the wave functions of the free ion (section 3.3) are the parity 
TABLE 16. Reduced matrix elements

\begin{tabular}{|c|c|c|c|c|c|c|}
\hline Element & $L$ & $S$ & $J$ & $\nu=2$ & $\nu=4$ & $\nu=6$ \\
\hline \multirow[t]{2}{*}{$\begin{array}{l}\mathrm{Ce}^{+++} \\
\mathrm{Yb}^{+++}\end{array}$} & \multirow[t]{2}{*}{3} & \multirow[t]{2}{*}{$1 / 2$} & & -0.325732 & 0. 360856 & -0.491065 \\
\hline & & & $\begin{array}{l}5 / 2 \\
7 / 2\end{array}$ & $\begin{array}{l}-.301569 \\
-.307788\end{array}$ & $\begin{array}{l}.261168 \\
.289329\end{array}$ & $\stackrel{0}{-.245532}$ \\
\hline \multirow{2}{*}{$\begin{array}{l}\mathrm{Pr}^{+++} \\
\mathrm{Tm}^{+++}\end{array}$} & \multirow[t]{2}{*}{5} & \multirow[t]{2}{*}{1} & & -.276822 & -.261529 & . 455018 \\
\hline & & & $\begin{array}{l}6 \\
5 \\
4\end{array}$ & $\begin{array}{r}-.265205 \\
-.249840 \\
-.260040\end{array}$ & $\begin{array}{r}-.224931 \\
-.174353 \\
-.208628\end{array}$ & $\begin{array}{l}.321183 \\
.136505 \\
.261268\end{array}$ \\
\hline \multirow{3}{*}{$\begin{array}{l}\mathrm{Nd}^{+++} \\
\mathrm{Er}^{+++}\end{array}$} & \multirow[t]{3}{*}{6} & \multirow[t]{3}{*}{$3 / 2$} & & -.106082 & -.168698 & -.642369 \\
\hline & & & $\begin{array}{r}9 / 2 \\
11 / 2 \\
13 / 2\end{array}$ & $\begin{array}{r}-.098820 \\
-.091864 \\
-.093887\end{array}$ & $\begin{array}{r}-.131239 \\
-.096145 \\
-.106106\end{array}$ & $\begin{array}{r}-.356506 \\
-.099840 \\
-.169019\end{array}$ \\
\hline & & & $15 / 2$ & -.101524 & -.144946 & -.457704 \\
\hline \multirow[t]{3}{*}{$\begin{array}{l}\mathrm{Pm}^{+++} \\
\mathrm{Ho}^{+++}\end{array}$} & \multirow[t]{3}{*}{6} & \multirow[t]{3}{*}{2} & & .106082 & .168698 & .642369 \\
\hline & & & $\begin{array}{l}4 \\
5 \\
6\end{array}$ & $\begin{array}{l}.095469 \\
.084208 \\
.083901\end{array}$ & $\begin{array}{r}.115742 \\
.054866 \\
.061345\end{array}$ & $\begin{array}{r}.260356 \\
-.090686 \\
-.087596\end{array}$ \\
\hline & & & $\begin{array}{l}7 \\
8\end{array}$ & $\begin{array}{l}089965 \\
.100398\end{array}$ & $\begin{array}{l}088311 \\
.139515\end{array}$ & $\begin{array}{l}062990 \\
.420886\end{array}$ \\
\hline \multirow[t]{3}{*}{$\begin{array}{l}\mathrm{Sm}^{+++} \\
\mathrm{Dy}\end{array}$} & \multirow[t]{3}{*}{5} & \multirow[t]{3}{*}{$5 / 2$} & & 276822 & .261529 & -.455018 \\
\hline & & & $\begin{array}{l}5 / 2 \\
7 / 2 \\
9 / 2\end{array}$ & $\begin{array}{l}217802 \\
.160051 \\
.155288\end{array}$ & $\begin{array}{r}.102885 \\
-.033771 \\
-.038079\end{array}$ & $\begin{array}{r}0 \\
.252972 \\
.220158\end{array}$ \\
\hline & & & $\begin{array}{l}11 / 2 \\
13 / 2 \\
15 / 2\end{array}$ & $\begin{array}{l}.174559 \\
.208638 \\
.253811\end{array}$ & $\begin{array}{r}-.007942 \\
.070737 \\
.193261\end{array}$ & $\begin{array}{r}.210017 \\
.112678 \\
-.228851\end{array}$ \\
\hline \multirow[t]{3}{*}{$\begin{array}{l}\mathrm{Eu}^{+++} \\
\mathrm{Tb}^{+++}\end{array}$} & \multirow[t]{3}{*}{3} & \multirow[t]{3}{*}{3} & & .325732 & -.360856 & .491065 \\
\hline & & & $\begin{array}{l}0 \\
1 \\
2\end{array}$ & $\begin{array}{l}0 \\
-.199469 \\
-.012363\end{array}$ & $\begin{array}{l}0 \\
0 \\
-.150786\end{array}$ & $\begin{array}{l}0 \\
0 \\
0\end{array}$ \\
\hline & & & $\begin{array}{l}3 \\
4 \\
5 \\
6\end{array}$ & $\begin{array}{r}-.054289 \\
.032147 \\
.138409 \\
.265202\end{array}$ & $\begin{array}{r}.060143 \\
.171347 \\
.130764 \\
-.168697\end{array}$ & $\begin{array}{r}-.081844 \\
.259348 \\
-.227510 \\
.064237\end{array}$ \\
\hline $\mathrm{Gd}^{+++}$ & 0 & $7 / 2$ & & 0 & 0 & 0 \\
\hline
\end{tabular}

Note 1: Where no $J$ value is given, the tabulated quantity is $\left(\beta L S\left\|_{\|} \mathbf{Y}_{\nu}\right\| \beta L S\right)$, otherwise $\left(\beta L S J\left\|Y_{\nu}\right\| \beta L S J\right)$.

Note 2: For the second ion of the above pairs, all signs change.

" $g$ " or " $u$ ", the total angular momentum $J$ and its component $M$. 'The strict selection rules $\Delta J=0, \pm 1(0+\rightarrow 0)$ for electric and magnetic dipole radiation, and $\Delta J=0, \pm 1, \pm 2(0 \nmid \rightarrow 0,0+\rightarrow 1)$ for electric quadrupole radiation, with change in parity in the first case, no change in the last two, were calculated in section 4.4. There is no physical significance to a selection rule on $M$ in the absence of a nonspherical perturbation. If the spin-orbit interaction is small, $L$ and $S$ approximately describe a state and we obtain selection rules in $L$ similar to those in $J$, while in $S$ we obtain $\Delta S=0$ for electric dipole or quadrupole transitions, $\Delta S=0, \pm 1 \quad(0 \nrightarrow 0)$ for magnetic dipole transitions. Thus, consider a transition probability for electric dipole radiation involving the matrix element $\left\langle\beta^{\prime} L^{\prime} S J^{\prime} M^{\prime}|e \mathbf{r}| \beta L S J M\right\rangle$. If $J^{\prime}=J+1$ so the transition is allowed in $J$, but $L^{\prime}=L+2$, it will be forbidden in $L$, and will be nonzero only if the spin-orbit interaction has mixed terms with spatial angular momentum $L+1$ in either $L$ or $L^{\prime}$ or both. The application of a magnetic field splits a state into its $2 J+1$ components with a splitting determined to a first approximation by the matrix element of $-\frac{e}{2 m c}(\mathbf{L}+2 \mathrm{~S}) \cdot \mathbf{B}$, yielding the usual expression for the Landé $g$-factor. Selection rules for $M$ now become sig- 
nificant, and we have $\Delta M=0, \pm 1$ for either type of dipole radiation, $\Delta M=0, \pm 1, \pm 2$ for quadrupole transitions, and their associated polarization effects.

The perturbing potential $V(2.6)$ will influence a state $|\alpha J M\rangle$ in general by four types of matrix elements, the effects of which we shall examine in detail. These are:

1. Matrix elements diagonal in $\alpha, J, M$.

2. Matrix elements diagonal in $\alpha, J$.

3. Matrix elements diagonal in $\alpha$.

4. Matrix elements nondiagonal in $\alpha$, though perhaps diagonal in $J$ and $M$. These matrices (or rather, approximations to them) may be calculated by the methods of section 5, though the scope of table 15 itself will suffice only for elements of types (1), (2), and those of (4) diagonal in parity and $J$. We will now examine the effect on the original level of these several matrix elements.

Matrix elements of type (1) arise from potential terms with $\nu$ even, $\mu=0$. Their effect is to split the level into pairs of states $\pm M$ (and the single level $M=0$ for integral $J$ in the ratios indicated by table 15 . The inclusion of these matrix elements only results in an initial axial field approximation which is sometimes useful. The selection rules on $J$ and parity, as well as any approximate rules on $L$ and $S$, remain valid, as do those on $M$ corresponding to the free ion in a magnetic field. This case corresponds closely to a reduction in symmetry from $D_{J}$ to $\mathrm{D}_{\infty}$, which differs through the inclusion of matrix elements of types (3) and (4) which are diagonal in $M$.

Elements of type (2) arise from potential terms with $\nu$ even but $\mu \neq 0$, still with the ratios determined by table 15 and of the same order of magnitude as those of type (1). The solution of the secular determinant for elements of (1) and (2) together will yield the first approximation to the energies of the perturbed states as well as the zero-order linear combination of states with various $M$ required for higher order perturbation calculations. States with $M$ values differing by $\mu$ will interact and $M$ will no longer be a good quantum number for these states. However, if $2 J$ is less than the lowest $\mu$ involved, there will be no matrix elements of this type and the rules for (1) only will still be valid. This situation will occur for levels of low $J$ in fields of high symmetry. Generally, the selection rules for $J$ and $\alpha$ will still hold. Selection rules on $M$ will apply to those states which do not involve a combination of $M$ values, and can be applied to the components of those states which do involve a mixing of two or more $M$ states.

Type (3) matrix elements still involve only potential terms with $\nu$ even, since $\alpha$ includes the parity quantum number. The addition of these matrix elements to our scheme destroys the validity of $J$ as a quantum number which has held for (1) and (2). These elements are those between various $J$ values of a given $L-S$ term, since they are still diagonal in $\alpha$. In order of magnitude, these elements are as large as those of types (1) and (2), since all three come from the same reduced $L-S$ matrix element (table 16) and differ only in the associated Racah and Wigner coefficients $(5.14,5.4)$. Their effect is numerically somewhat smaller, since terms they contribute in perturbation theory are divided by the energy differences between the unperturbed levels. A state of given $J=J_{0}$ will be mixed directly with other $J$ values such that $\left|J-J_{0}\right| \leq \nu$. The selection rules for the free ion $J$ are now either poor or completely worthless, depending on the numerical parameters. Since both the upper and lower states of a transition can involve a range of $J$, the effective selection rule is merely $\Delta J \leq 2 \nu+1$.

Matrix elements of type (4) will generally be numerically the smallest of the four types. They arise from all terms in the potential (2.6), this being the only one of the four where the terms with odd $\nu$ are included. Such elements, at least as far as the ground state is concerned, involve relatively distant levels, so the influence on the splitting of the ground state will be small and lie perhaps more in the overall position of the level with respect to the unperturbed level. Perhaps the most important effect of such elements is on the selection rules and the associated polarization effects, and in the intensity calculations. Just as elements of type (3) destroyed $J$ as a quantum number, so will these destroy $\alpha$ as a quantum number. $\alpha$ includes the specification of the configuration and consequently the parity of a state. In the most general case, there will be matrix elements for even $\nu$ between $4 f^{n}$ and higher even configurations, such as $4 f^{n-2} 5 d^{2}$, and for odd $\nu$ between $4 f^{n}$ and $4 f^{n-1} 5 d$. These latter elements destroy the parity of the state and lift the restrictions of Laporte's rule for electric dipole transitions. Intensities corresponding to electric dipole transitions between states principally $4 f^{n}$ in character must, therefore, be computed from these extremely small inter-configurational matrix elements and are therefore extremely weak; nevertheless, all crystal spectra are in any absolute sense quite weak and such transitions must be considered in the case of any nonholohedric perturbing potential.

The further effect of a uniform magnetic field will depend to a large extent on the degeneracy allowed by the electrostatic perturbation. The magnetic field does not allow any remaining degeneracy; hence, levels degenerate in the electrostatic field, including the Kramers conjugate states, will be split by a term linear in the magnitude of the field $B$ which can be computed from a solution of the associated secular determinant. The average magnetic moment of any nondegenerate state is zero (Van Vleck, 1932; Klein, 1952) so these states will show no first order effect for weak magnetic fields. If the crystal field leaves two or more levels relatively close together, a case of intermediate or strong magnetic field approximation can be observed and the level will show a 
finite displacement from its $B=0$ position. Unless the magnetic field and crystal field symmetry axes are parallel, there will generally be no symmetry and no selection rules. Marked variation of intensities of lines has been observed in the crystal Zeeman effect. A more specific example of some of these considerations will be given in section 6.2.

\subsection{A Crystal Field}

The direct approach to a crystal problem is to obtain the crystal structure by diffraction measurements, geometric considerations, or other crystallographic techniques. These techniques do not locate the lighter ions with sufficient accuracy, particularly water of crystallization, which contribute greatly to the crystal field. As an illustration of the procedure, let us consider a possible short range structure for rare-earth chlorides of the form $\mathrm{XCl}_{3} \cdot 6 \mathrm{H}_{2} \mathrm{O}$ (H. S. Robertson, informal communication) where $6 \mathrm{Cl}^{-}$(three from adjoining $X)$ are in two horizontal planes equidistant from $X$, forming an equilateral triangle in each plane, the two triangles being rotated $60^{\circ}$ to each other about a vertical axis. The water molecules [considered as dipoles] are then located along the $X-\mathrm{Cl}$ lines.

While detailed calculations were not carried out due to uncertainties in the various distance parameters, certain features of considerable interest may be obtained through partial application of (2.7). The above charge array may be described by

$$
\begin{aligned}
& \alpha_{1-9}=\theta \quad \alpha_{10-18}=\pi-\theta \quad \beta_{1-3}=0 \\
& \beta_{4-6}=\frac{2 \pi}{3} \quad \beta_{7-9}=\frac{4 \pi}{3} \quad \beta_{10-12}=\frac{\pi}{3} \quad \beta_{13-15}=\pi \\
& \beta_{16-18}=\frac{5 \pi}{3} \quad q_{3 n}=q_{3 n+1}=-e \quad q_{3 n+2}=e
\end{aligned}
$$

and we shall take $\theta$ as a variable parameter. There are a total of 18 charges in this array. Those above the $x-y$ plane have the indices $1-9$, while those below have 10-18. Indices of the form $3 n$ refer to $\mathrm{Cl}^{-}, 3 n+1$ refers to $\mathrm{OH}^{-}$and $3 n+2$ refers to $\mathrm{H}^{+}$. The angular portions of the potential were calculated over a range from $\theta=0^{\circ}$ to $90^{\circ}$. The angular factors (2.4) were obtained from the tables of Tallqvist (1908). This table is not widely available, but no other table covering its range has been prepared to the knowledge of the writer. The National Bureau of Standards table (1945) covers values of $M$ only up to and including 4 .

In performing the summation (2.7), the only generally nonvanishing coefficients are $(N \leq 6)$ $C_{2}^{0}, C_{4}^{0}, C_{6}^{0}, C_{4}^{3}, C_{6}^{3}$, and $C_{6}^{6}$, though this may be deduced directly from table 5 without explicitly performing the summation. The values of these, apart from a radial factor of the form

$$
e\left(\frac{-1}{R_{1}^{N+1}}+\frac{-1}{R_{2}^{N+1}}+\frac{1}{R_{3}^{N+1}}\right)
$$

are given in table 17. Certain general qualitative features may be noted by inspection. For $\theta=0$ (corresponding to all charges being located along the vertical axis), the symmetry is $\mathrm{D}_{\infty h}$ (table 5). For other angles, the symmetry is generally $\mathrm{D}_{3 d}$, while at $90^{\circ}$ (a planar configuration) it is $\mathrm{D}_{6} h$. A particular exception occurs for $\theta=54^{\circ} 44^{\prime}$ where the symmetry is "accidentally" $\mathrm{O}_{h}$, belonging to the cubic point groups (section 2.4). For this value of $\theta$, the three pairs of charge axes are mutually perpendicular and the $C_{2}^{0}$ term vanishes. For other values of $\theta\left(\right.$ e.g., $\left.31^{\circ}\right)$, other coefficients vanish, but there is no general theoretical significance to this. It is to be noted that for $\theta<10^{\circ}$, the terms responsible for departure from $D_{\infty h}$ symmetry are small, while in the vicinity of $\theta=90^{\circ}$ the terms causing departure from $\mathrm{D}_{6 h}$ symmetry are large and quite sensitive to small angular variations.

Most features of even this simple problem require the specification of the radial distances of the ions, as well as the average radial integrals $\left\langle r^{v}\right\rangle$ of the wave functions. On the other hand, aspects of the Zeeman effect can be understood without further knowledge of these parameters,

\begin{tabular}{|c|c|c|c|c|c|c|}
\hline$\theta$ & $C_{2}^{0}$ & $C^{0}$ & $C_{6}^{0}$ & $C_{4}^{3}$ & $C_{6}^{3}$ & $C_{6}^{6}$ \\
\hline $0^{\circ}$ & 9.5120 & 7. 0898 & 5. 8991 & 0 & 0 & \\
\hline $10^{\circ}$ & 9.0818 & 6. 0491 & 4. 1557 & 0.0765 & 0.2113 & 0.0001 \\
\hline $20^{\circ}$ & 7. 8430 & 3. 3675 & 0.4242 & .5575 & 1. 3485 & .006 \\
\hline $30^{\circ}$ & 5. 9450 & 0.1662 & -2.2064 & 1. 6053 & 3. 0365 & 0619 \\
\hline $40^{\circ}$ & 3. 6168 & -2.2617 & -1.9088 & 3.0170 & 3. 7557 & 279 \\
\hline $45^{\circ}$ & 2. 3780 & -2.8802 & -0.8756 & 3. 7074 & 3. 3393 & 495 \\
\hline $50^{\circ}$ & 1. 1392 & -3.0311 & .3326 & 4. 2850 & 2. 3851 & .8007 \\
\hline${ }^{a} \mathrm{O}_{h}$ & & -2.7571 & 1. 3109 & 4. 6604 & 0.7915 & 1. 174 \\
\hline $60^{\circ}$ & -1.1890 & -2.0494 & 1. 9068 & 4. 8160 & -.4338 & 1. 67 \\
\hline $70^{\circ}$ & -3.0870 & -0.0269 & 1. 2322 & 4. 2086 & -2.5978 & 2.728 \\
\hline $80^{\circ}$ & -4.3258 & 1. 8852 & -0.7794 & 2. 4595 & -2.3645 & 3. 6146 \\
\hline $90^{\circ}$ & -4.7560 & 2. 6587 & -1.8435 & & & 3. 9624 \\
\hline
\end{tabular}
and these aspects are most important for understanding more complicated cases. Let us assume

TABLE 17. A representative crystal field

a See text. 
that we have the case $\theta=0^{\circ}$ of the preceding table 17 , i.e., $\mathrm{D}_{\infty h}$ symmetry. We see from table 9 that the $2 J+1$ states are separated into doubly degenerate pairs corresponding to $\pm M$, plus (for integral $J$ only) a nondegenerate state $M=0$.

The application of a uniform magnetic field parallel to the $z$-axis will reduce the symmetry to $C_{\infty} h$ and all levels are now nondegenerate. The splitting is given by twice the diagonal matrix element $\left\langle M\left|L_{z}+2 S_{z}\right| M\right\rangle B$, since the $-M$ level is depressed by this same amount and there are no nondiagonal elements, $L_{z}+2 S_{z}$ having no dependence on $\phi$. If the field is applied parallel to the $y$ axis, the only symmetry remaining is $C_{2 h}$, but this is referred to a horizontal axis, rather than a vertical one. The secular determinant will now involve matrix elements of $L_{y}+2 S_{y}$. This has a $\phi$ dependence of the form $e^{ \pm i \phi}$, and will have nonvanishing matrix elements between states differing in $M$ by 1 . Thus, of all the $\pm M$ pairs, a transverse magnetic field will have no diagonal elements and will have nondiagonal elements only in the case of $\pm \frac{1}{2}$. The magnitude of this element will be $\frac{1}{2} \sqrt{\left(J+\frac{1}{2}\right)\left(J-\frac{1}{2}+1\right)} g \beta B$ and the splitting will be twice that or $\left(J+\frac{1}{2}\right) g \beta B$.

If we now proceed to the planar case $D_{6 h}$, we see from table 10 that for $J \leq \frac{5}{2}$ the states remain as in the $D_{\infty h}$ case and the Zeeman effect for these lower $J$ values is unchanged. For $J=3$, the original pair $M= \pm 3\left(E_{3 g, 3 u}\right)$ now splits into two nondegenerate states $B_{1 g, 1 u}$ and $B_{2 g, 2 u}$, which are the states $\frac{1}{\sqrt{2}}\left|+3>+\frac{1}{\sqrt{2}}\right|-3>$ and $\frac{1}{\sqrt{2}} \mid+3>$

$-\frac{1}{\sqrt{2}} \mid-3>$, respectively. This arises from the $e^{ \pm 6 i \phi}$ in the potential with nonzero matrix elements between terms where $\Delta M= \pm 6$. These states will show no first-order Zeeman effect, either longitudinal or transverse. For $J=4$, we see that the states coming from \pm 2 and \pm 4 have the same symmetry $\left(E_{2 g, 2 u}\right)$, and while we still have degenerate pairs, their properties will depend upon the solution of the associated secular determinant. One pair will be given by an expression of the form $a\left| \pm 4>+\left(1-a^{2}\right)^{\frac{1}{2}}\right| \mp 2>$ while the other will be of the form $\left(1-a^{2}\right)^{\frac{1}{2}}| \pm 4>-a| \mp 2>$. The coefficient $a$ will be fixed by the magnitude of $C_{6}^{6}$. For these terms, the longitudinal Zeeman splitting will be $2\left[4 a^{2}-2\left(1-a^{2}\right)\right]=12 a^{2}-4$ and $2\left[4\left(1-a^{2}\right)\right.$ $\left.-2 a^{2}\right]=12 a^{2}-8$, respectively, while the transverse effect remains zero. This longitudinal effect thus depends explicitly on the crystal field. For $J=\frac{7}{2}$, a similar phenomenon takes place through a combination of $\mid \pm \frac{7}{2}>$ with $\mid \mp \frac{5}{2}>$, and the longitudinal effect will again depend explicitly on the crystal field. A major difference will occur in the transverse effect-here there are nonvanishing off-diagonal matrix elements of the form $<\frac{7}{2} L_{y}+2 S_{y}\left|\frac{5}{2}\right\rangle$, in the secular determinant and there will be a transverse Zeeman splitting, also depending explicitly on the crystal field. Under. still lower symmetries than $\mathrm{D}_{6 n}$, the Zeeman effect in odd-electron systems becomes increasingly dependent on the crystal field, since Kramers degeneracy remains in all cases, or vanishes in first order in most even-electron systems where the crystal field states generally become nondegenerate.

\subsection{The Fluorescence Spectrum of the Terbium Ion}

Because of the inexact knowledge of the radial parameters necessary for further work on the direct approach to a solution of a given problem, the more common procedure is to utilize the quantities $\left\langle r^{N}\right\rangle C_{N}^{M}$ and $\left\langle r^{N}\right\rangle S_{N}^{M}$ as adjustable parameters to fit as best as possible the observed data. This leaves the radial wave function integrals combined with the crystal field parameters-they are frequently left in this form since further information about either one of them separately is not readily available. We shall illustrate this procedure by some considerations of the fluorescence spectrum of $\mathrm{TbCl}_{3} \cdot 6 \mathrm{H}_{2} \mathrm{O}$.

The experimentally observed fluorescence lines (Singh, J.H. U., Dissertation, 1957), observed both at $4.2^{\circ} \mathrm{K}$ and $2.2^{\circ} \mathrm{K}$, are given in table 18 . The exciting light, unpolarized, is incident along the crystal axis (the $z$ axis). For both temperatures, the spacing of each level from the lowest of its group is given, the average of the two temperature values, and the spacing of this (to the nearest $\mathrm{cm}^{-1}$ ) from the mean of the group. The overall mean for each group is also given. The fluorescent light is observed on the side of the crystal opposite the incident radiation, also along the $z$-axis. The fluorescent lines are relatively weak and are generally observed without regard to polarization; however, a few plates were taken at $4.2^{\circ} \mathrm{K}$ showing polarization (electric vector along $x$ or $y$ ). These data were made available by private communication from Dr. Singh and are also included in table 18. In the interpretation of fluorescence spectra it should be remembered that the spectra may originate near the surface of the crystal, and that local fields, different from those in the main body of the crystal, may significantly affect the regularities expected.

The Tb ion has the configuration $4 f^{8}$. According to Hund's rule, the ground state is a ${ }^{7} F$ multiplet with $J=6$ lowest, with $J$ from 5 to 0 above the lowest. The fluorescence spectrum is caused by transitions from a higher level (probably ${ }^{5} \mathrm{D}$ but this is not important for the present considerations) to various levels of the ground multiplet. Qualitatively, the agreement with experiment is excellent. The groups of lines $Z, Y, X, W, V, U$, $T$, correspond to $J=6,5,4,3,2,1$, and 0 , in that order. Within each group, there are $2 J+1$ lines, indicating that the crystal field has removed all degeneracy. This is confirmed by the absence of any first order Zeeman effect. 
TABLE 18. Fluorescence spectrum of $\mathrm{TbCl}_{3} \cdot 6 \mathrm{H}_{2} \mathrm{O}$

\begin{tabular}{|c|c|c|c|c|c|c|c|c|}
\hline \multirow{2}{*}{ Line } & \multicolumn{2}{|c|}{$\left(4.2^{\circ} \mathrm{K}\right)$} & \multicolumn{2}{|c|}{$\left(2.2^{\circ} \mathrm{K}\right)$} & \multirow[b]{2}{*}{$\overline{\Delta \nu}$} & \multirow[b]{2}{*}{$\overline{\Delta \nu}-\Delta \nu_{m}$} & \multirow[b]{2}{*}{$x y$} & \\
\hline & $\nu$ & $\Delta \nu$ & $\nu$ & $\Delta \nu$ & & & & \\
\hline $\begin{array}{l}Z_{1} \\
Z_{2}\end{array}$ & $\begin{array}{l}20538.81 \\
20503.66\end{array}$ & $\begin{array}{r}0.00 \\
35.15\end{array}$ & $\begin{array}{r}20538.77 \\
503.72\end{array}$ & $\begin{array}{r}0.00 \\
35.05\end{array}$ & $\begin{array}{r}0.00 \\
35.10\end{array}$ & $\begin{array}{l}-167 \\
-132\end{array}$ & \multirow[b]{2}{*}{$\begin{array}{l}\text { No } \\
\text { data }\end{array}$} & \multirow{7}{*}{$\bar{Z}=20372$} \\
\hline $\begin{array}{l}Z_{3} \\
Z_{4}\end{array}$ & $\begin{array}{l}\text { 498. } 20 \\
461.26\end{array}$ & $\begin{array}{l}40.61 \\
77.55\end{array}$ & $\begin{array}{l}498.33 \\
461.59\end{array}$ & $\begin{array}{l}40.44 \\
77.18\end{array}$ & $\begin{array}{l}40.52 \\
77.36\end{array}$ & $\begin{array}{r}-127 \\
-90\end{array}$ & & \\
\hline $\begin{array}{l}Z_{5} \\
Z_{6}\end{array}$ & 380. 70 & 158.11 & $\begin{array}{l}\text { 454. } 97 \\
\text { 379. } 70\end{array}$ & $\begin{array}{r}83.80 \\
159.07\end{array}$ & $\begin{array}{r}83.80 \\
158.59\end{array}$ & $\begin{array}{r}-83 \\
-8\end{array}$ & & \\
\hline $\begin{array}{l}Z_{7} \\
Z_{8}\end{array}$ & $\begin{array}{l}369.16 \\
359.49\end{array}$ & $\begin{array}{l}169.65 \\
179.32\end{array}$ & $\begin{array}{l}370.19 \\
359.27\end{array}$ & $\begin{array}{l}168.58 \\
179.50\end{array}$ & $\begin{array}{l}169.12 \\
179.41\end{array}$ & $\begin{array}{r}2 \\
12\end{array}$ & & \\
\hline $\begin{array}{l}Z_{9} \\
Z_{10}\end{array}$ & 306. 14 & 232.67 & $\begin{array}{l}\text { 347. } 85 \\
305.73\end{array}$ & $\begin{array}{l}\text { 190. } 92 \\
\text { 233. } 04\end{array}$ & $\begin{array}{l}\text { 190. } 92 \\
232.86\end{array}$ & $\begin{array}{l}24 \\
66\end{array}$ & & \\
\hline $\begin{array}{l}Z_{11} \\
Z_{12}\end{array}$ & $\begin{array}{l}239.08 \\
193.84\end{array}$ & $\begin{array}{l}299.73 \\
344.95\end{array}$ & $\begin{array}{l}\text { 238. } 33 \\
\text { 192. } 86\end{array}$ & $\begin{array}{l}300.44 \\
345.91\end{array}$ & $\begin{array}{l}300.08 \\
345.43\end{array}$ & $\begin{array}{l}133 \\
178\end{array}$ & & \\
\hline$Z_{13}$ & 179.61 & 359. 20 & 179. 33 & 359.44 & 359.32 & 192 & & \\
\hline $\begin{array}{l}Y_{1} \\
Y_{2}\end{array}$ & $\begin{array}{r}18433.00 \\
413.02\end{array}$ & $\begin{array}{r}0.00 \\
19.98\end{array}$ & $\begin{array}{r}18433.04 \\
413.02\end{array}$ & $\begin{array}{r}0.00 \\
20.00\end{array}$ & $\begin{array}{r}0.00 \\
19.99\end{array}$ & $\begin{array}{l}-127 \\
-107\end{array}$ & $\begin{array}{ll}\mathrm{S} & \mathrm{w} \\
\mathrm{S} & \mathrm{w}\end{array}$ & \multirow{5}{*}{$\bar{Y}=18306$} \\
\hline $\begin{array}{l}Y_{3} \\
Y_{4}\end{array}$ & $\begin{array}{l}400.92 \\
359.26\end{array}$ & $\begin{array}{l}32.08 \\
73.74\end{array}$ & $\begin{array}{l}400.99 \\
359.41\end{array}$ & $\begin{array}{l}32.05 \\
73.63\end{array}$ & $\begin{array}{l}32.06 \\
73.68\end{array}$ & $\begin{array}{l}-95 \\
-53\end{array}$ & $\begin{array}{cc}\mathrm{w} & \mathrm{s} \\
\mathrm{s} & \mathrm{w}\end{array}$ & \\
\hline $\begin{array}{l}Y_{5} \\
Y_{6}\end{array}$ & 341.00 & 92.00 & $\begin{array}{l}341.09 \\
333.69\end{array}$ & $\begin{array}{l}91.95 \\
99.35\end{array}$ & $\begin{array}{l}91.98 \\
99.35\end{array}$ & $\begin{array}{l}-35 \\
-27\end{array}$ & $\begin{array}{ll}\text { S } & \text { w } \\
\text { S } & \text { W }\end{array}$ & \\
\hline $\begin{array}{l}Y_{7} \\
Y_{8}\end{array}$ & $\begin{array}{l}308.29 \\
294.96\end{array}$ & $\begin{array}{l}\text { 124. } 71 \\
138.04\end{array}$ & $\begin{array}{l}308.57 \\
295.90\end{array}$ & $\begin{array}{l}124.47 \\
137.14\end{array}$ & $\begin{array}{l}124.59 \\
137.59\end{array}$ & $\begin{array}{r}-2 \\
11\end{array}$ & $\begin{array}{ll}\mathrm{s} & \mathrm{W} \\
\mathrm{S} & \mathrm{W}\end{array}$ & \\
\hline $\begin{array}{l}Y_{9} \\
Y_{10}\end{array}$ & $\begin{array}{l}250.87 \\
124.83\end{array}$ & $\begin{array}{l}\text { 182. } 13 \\
308.17\end{array}$ & 124.82 & 308.22 & $\begin{array}{l}\text { 182. } 13 \\
308.20\end{array}$ & $\begin{array}{r}55 \\
181\end{array}$ & $\begin{array}{l}\text { S W } \\
? ?\end{array}$ & \\
\hline$Y_{11}$ & 107. 90 & 325.10 & 107. 81 & 325.23 & 325.16 & 198 & $? ?$ & \multirow{5}{*}{$\bar{X}=17120$} \\
\hline $\begin{array}{l}X_{1} \\
X_{2}\end{array}$ & 17193.56 & 0.00 & $\begin{array}{r}17193.49 \\
188.20\end{array}$ & $\begin{array}{l}0.00 \\
5.29\end{array}$ & $\begin{array}{l}0.00 \\
5.29\end{array}$ & $\begin{array}{l}-74 \\
-69\end{array}$ & $\begin{array}{l}\mathrm{w} \quad \mathrm{w} \\
\mathrm{W}\end{array}$ & \\
\hline $\begin{array}{l}X_{3} \\
X_{4}\end{array}$ & 184.65 & 8. 91 & $\begin{array}{l}\text { 184. } 76 \\
135.74\end{array}$ & $\begin{array}{r}8.73 \\
57.75\end{array}$ & $\begin{array}{r}8.82 \\
57.75\end{array}$ & $\begin{array}{l}-65 \\
-16\end{array}$ & $\begin{array}{l}\mathrm{s} \\
\mathrm{s} \mathrm{w}\end{array}$ & \\
\hline $\begin{array}{l}X_{5} \\
X_{6}\end{array}$ & 131. 91 & 61.65 & $\begin{array}{l}\text { 131. } 98 \\
104.88\end{array}$ & $\begin{array}{l}61.51 \\
88.61\end{array}$ & $\begin{array}{l}61.58 \\
88.61\end{array}$ & $\begin{array}{r}-12 \\
15\end{array}$ & $\begin{array}{l}\mathrm{S} \mathrm{w} \\
\mathrm{vW}\end{array}$ & \\
\hline $\begin{array}{l}X_{7} \\
X_{8}\end{array}$ & 067.31 & 126. 25 & $\begin{array}{l}067.52 \\
056.26\end{array}$ & $\begin{array}{l}\text { 125. } 97 \\
\text { 137. } 23\end{array}$ & $\begin{array}{l}\text { 126. } 11 \\
137.23\end{array}$ & $\begin{array}{l}52 \\
63\end{array}$ & " " & \\
\hline$X_{9}$ & 013. 94 & 179. 62 & 014.48 & 178.81 & 179. 22 & 105 & “ & \multirow{5}{*}{$\bar{W}=16065$} \\
\hline $\begin{array}{l}W_{1} \\
W_{2}\end{array}$ & 16162. 72 & 0.00 & $\begin{array}{r}16162.85 \\
080.68\end{array}$ & $\begin{array}{r}0.00 \\
82.17\end{array}$ & $\begin{array}{r}0.00 \\
82.17\end{array}$ & $\begin{array}{l}-98 \\
-15\end{array}$ & $\begin{array}{l}\mathrm{W} \\
\mathrm{S}\end{array}$ & \\
\hline $\begin{array}{l}W_{3} \\
W_{4}\end{array}$ & $\begin{array}{l}\text { 070. } 26 \\
043.44\end{array}$ & $\begin{array}{r}92.46 \\
119.28\end{array}$ & $\begin{array}{l}070.33 \\
043.44\end{array}$ & $\begin{array}{r}92.52 \\
119.41\end{array}$ & $\begin{array}{r}92.49 \\
119.34\end{array}$ & $\begin{array}{r}-5 \\
22\end{array}$ & $\begin{array}{l}\text { w w } \\
\text { W s }\end{array}$ & \\
\hline $\begin{array}{l}W_{5} \\
W_{6}\end{array}$ & $\begin{array}{l}037.91 \\
032.99\end{array}$ & $\begin{array}{l}124.81 \\
129.73\end{array}$ & $\begin{array}{l}037.95 \\
032.87\end{array}$ & $\begin{array}{l}124.90 \\
129.98\end{array}$ & $\begin{array}{l}124.86 \\
129.86\end{array}$ & $\begin{array}{l}27 \\
32\end{array}$ & $\begin{array}{l}\mathrm{W} \text { w } \\
\mathrm{S} \text { w }\end{array}$ & \\
\hline$W_{7}$ & 028.07 & 134.65 & 028. 12 & 134. 69 & 134. 69 & 37 & $\mathrm{~s} \mathrm{w}$ & \\
\hline $\begin{array}{l}V_{1} \\
V_{2}\end{array}$ & $\begin{array}{r}15499.89 \\
430.03\end{array}$ & $\begin{array}{r}0.00 \\
69.86\end{array}$ & \multirow{6}{*}{15253.17} & & & $\begin{array}{r}-145 \\
-75\end{array}$ & \multirow{6}{*}{$\begin{array}{l}\mathrm{VW} \mathrm{S} \\
\mathrm{s} \mathrm{VW} \\
\mathrm{VW} \mathrm{S}\end{array}$} & \multirow{3}{*}{$\bar{V}=15355$} \\
\hline $\begin{array}{l}V_{3} \\
V_{4}\end{array}$ & $\begin{array}{l}297.53 \\
292.94\end{array}$ & $\begin{array}{l}\text { 202. } 36 \\
206.95\end{array}$ & & & & $\begin{array}{l}57 \\
62\end{array}$ & & \\
\hline$V_{5}$ & 253. 19 & 246. 70 & & & & 101 & & \\
\hline $\begin{array}{l}U_{1} \\
U_{2}\end{array}$ & $\begin{array}{l}\text { 15001. } 25 \\
14994.68\end{array}$ & $\begin{array}{l}0.00 \\
6.57\end{array}$ & & & & $\begin{array}{l}-23 \\
-17\end{array}$ & & $\bar{U}=14978$ \\
\hline$U_{3}$ & 938.41 & 62.84 & & & & 40 & & \\
\hline$T$ & 14691. 73 & & & & & & & $\bar{T}=14692$ \\
\hline
\end{tabular}


Let us consider the quantitative details. For best results, our theory requires $L-S$ coupling and a crystal field splitting small compared to the multiplet separation. We may check the validity of $L-S$ coupling by the interval rule, taking the separation of the mean of adjacent groups and dividing by the higher $J$ value. We obtain, in $\mathrm{cm}^{-1}$, the following:

$$
\begin{array}{llllll}
J=6 & 344 & J=4 & 264 & J=2 & 158.5 \\
J=5 & 237 & J=3 & 237 & J=1 & 286
\end{array}
$$

This is not especially impressive for very good obedience to $L-S$ coupling requirements. Let us also calculate the total spread of a group, divided by the separation of the group mean from that for the next lower $J$. We obtain:

$$
\begin{array}{rrrrrr}
J=6 & 0.174 & J=4 & 0.170 & J=2 & 0.653 \\
J=5 & .274 & J=3 & .190 & J=1 & .220
\end{array}
$$

This indicates that the influence of adjacent levels may be rather significant. The irregularity here for $J=2$ is quite interesting; it is only partially due to the irregularity shown in the interval rule check, since the total spread of $J=2$ is $247 \mathrm{~cm}^{-1}$ while that of $J=3$ is only $179 \mathrm{~cm}^{-1}$.

In beginning an analysis, we must first obtain some idea of the symmetry. From table 9, we see that the only symmetries giving no degeneracy for an even-electron system are $\mathrm{D}_{2 h}, \mathrm{C}_{2 v}, \mathrm{C}_{2 h}$, and $\mathrm{C}_{s}$. The overall crystal symmetry suggests either $\mathrm{C}_{2 n}$ or $\mathrm{C}_{2 h}$. From table 5, we see that $\mathrm{D}_{2 h}$ requires the following potential coefficients: $C_{2}^{0} C_{4}^{0} C_{6}^{0} C_{2}^{2} C_{4}^{2} C_{6}^{2} C_{4}^{4} C_{6}^{4} C_{6}^{6}$. The lower symmetry $\mathrm{C}_{2 v}$ is obtained by the inclusion of odd potential terms, which will influence the energy splittings by a very small amount (section 6.1), while $\mathrm{C}_{2 n}$ will include even $S$ terms. These latter will strongly influence the energy splittings. In order to reduce the number of independent parameters, we shall assume $\mathrm{D}_{2 h}$, which will be energetically equivalent to $\mathrm{C}_{2 v}$.

In deducing the field parameters, we need to know not only the energies of the levels but also the free ion $M$ values from which they come. Where the crystal field does not remove all degeneracy, this may be partially obtained from the Zeeman splittings. For the present case, the only source of such information is the polarization data.

We begin our detailed analysis with the simplest nontrivial case $J=1$. We have three levels at $-23,-17$, and +40 . The only potential terms of $\mathrm{D}_{2 h}$ contributing to matrix elements within $J=1$ are $C_{2}^{0}$ and $C_{2}^{2}$. It is a natural first guess to consider that the $C_{2}^{0}$ term causes a splitting of the state $|0\rangle$ to +40 , and $|+1\rangle$ and $|-1\rangle$ to -20 , with a small splitting of the latter two to -17 and -23 by $C_{2}^{2}$. This is not necessarily the case, and must be in agreement with the polarization data.
From table 14 , where radiation in the $z$ direction corresponds to $\theta=\phi=0^{\circ}, \psi=0^{\circ}$ for $x$ polarization and $\psi=90^{\circ}$ for $y$ polarization, we see that $x$-polarized radiation may be produced by either $A_{x}^{\circ}, B_{y}$, or $A_{x z}$, while $y$-polarized radiation may be produced by $A_{y}^{\circ}, B_{x}$, or $A_{y z}$. These are the electric dipole, magnetic dipole, or electric quadrupole terms, respectively. We shall assume that the transitions are magnetic dipole in character. We do not know the symmetry of the upper state in the fluorescent spectrum, but there are only 4 possibilities in a $D_{2 h}$ symmetry. From table 13 we deduce the allowed lower state for each possible upper state as follows:

\begin{tabular}{c|c|c}
\hline \hline $\begin{array}{c}\text { Upper } \\
\text { state }\end{array}$ & $B_{y}\left(U_{1}\right)$ & $B_{x}\left(U_{2}\right)$ \\
\hline & & \\
\hline$A_{1 g}$ & $B_{2 g}$ & $B_{1 g}$ \\
$A_{2 g}$ & $B_{1 g}$ & $B_{2 g}$ \\
$B_{1 g}$ & $A_{2 g}$ & $A_{1 g}$ \\
$B_{2 g}$ & $A_{1 g}$ & $A_{2 g}$ \\
\hline
\end{tabular}

The identification of $U_{1}$ and $U_{2}$ of table 18 ideally requires that they be present in only one of the two polarizations, rather than "very weak," but the actual behavior is quite close to this ideal. We now turn to table 9 , and find that the lower state $1_{g}$ yields in $D_{2 h}$ symmetry the states $A_{2 g}$, $B_{1 g}$, and $B_{2 g}$. Since $A_{1 g}$ is not present in the lower group, we conclude that the upper state is either $A_{1 g}$ or $A_{2 g}$, that the lower states at -23 and -17 are $B_{1 g}$ and $B_{2 g}$, while the other state from $J=1$ is $A_{2 g}$. We cannot uniquely assign $B_{1 g}$ and $B_{2 g}$ to a particular one of the $-23,-17$ levels. The upper level is presumably ${ }^{5} D_{4}$ and a $4 \mathrm{~g}$ level under $\mathrm{D}_{2 h}$ gives both $A_{1 g}$ and $A_{2 g}$ levels. Interestingly enough, the same results, without " $g$ " and " $u$ " subscripts, hold for $\mathrm{C}_{2 v}$ symmetry. We now see from table 10 that $B_{1 g}$ and $B_{2 g}$ arise from the state $E_{\mathrm{Ig}}$ of $\mathrm{D}_{\infty} h$, i.e., they come from $M= \pm 1$ and our earlier guess is confirmed.

In order to achieve maximum simplicity in the calculations, it is in general desirable to use zeroorder wave functions which belong to reps of the symmetry group. In the present case, the state $\mid 0>$ belongs to $A_{2 g}$, but the states $\mid+1>$ and $\mid-1>$ are not appropriate, and we must use the (essentially real) combinations $B_{1 g}: \frac{1}{\sqrt{2}} \mid-1>+$ $\frac{1}{\sqrt{2}} \mid+1>$ and $B_{2 g}: \frac{1}{\sqrt{2}}\left|-1>-\frac{1}{\sqrt{2}}\right|+1>$. We shall frequently abbreviate these as $|1+\rangle$ and $|1-\rangle$, respectively. We now introduce the perturbation $V=r^{2}\left(C_{2}^{0} \mathrm{C}_{2}^{0}+C_{2}^{2} \mathrm{C}_{2}^{2}\right)$. Since the potential $V$ is symmetric under $D_{2 h}$ and our three zero order wave functions belong to different reps, all nondiagonal matrix elements vanish, and the energies are merely $\langle 0|V| 0\rangle,\langle 1+|V| 1+\rangle$ and $\langle 1-|V| 1-\rangle$. It is a general characteristic of 
crystal field problems that one must be freely able to shift from complex to real forms of wave functions and vice versa, as the symmetry demands.

In the present case, we have from (2.9) $Y_{2}^{0}=C_{2}^{0}$ and $Y_{2}^{2}=Y_{2}^{-2}=\frac{1}{\sqrt{2}} C_{2}^{2}$. Hence $\langle 0|V| 0\rangle=$

$\left(\alpha 1\left\|Y_{2}\right\| \alpha 1\right) C_{00}^{121} Y_{2}^{0}\left\langle r^{2}\right\rangle=40 \mathrm{~cm}^{-1}$ or, from tables 15 and $16,(0.199469)(-0.632456) \quad Y_{2}^{0}<r^{2}>=40$ $\mathrm{cm}^{-1}$, so that $Y_{2}^{0}\left\langle r^{2}\right\rangle=-317.1 \mathrm{~cm}^{-1}$. Similarly,

$$
\begin{aligned}
& \langle 1+|V| 1+\rangle=\left(\alpha 1\left\|Y_{2}\right\| \alpha 1\right)\left[\frac{1}{2}\left(C_{10}^{21}+C_{-10}^{121}\right) Y_{2}^{0}\right. \\
& \left.+\frac{1}{2}\left(C_{-12}^{121}+C_{1-2}^{1}\right) Y_{2}^{2}\right]\left\langle r^{2}\right\rangle=-17 \mathrm{~cm}^{-1} .
\end{aligned}
$$

Recalling (5.6) and using the value of $Y_{2}^{0}$ just computed above, we obtain (0.199469) (0.774597) $Y_{2}^{2}\left\langle r^{2}\right\rangle=+3$, or $Y_{2}^{2}=19.4 \mathrm{~cm}^{-1}$. The sign of $Y_{2}^{2}$ is ambiguous, since the state $|1+\rangle$ could equally well have been assigned to $-23 \mathrm{~cm}^{-1}$. These results, while interesting, are really not too profound, since we have only two independent energies relative to the mean of the group, and we had two adjustable coefficients at our disposal.

When we proceed to the $J=2$ case, we see that the allowed levels (table 9) are (2) $A_{1 \mathrm{~g}}, A_{2 \mathrm{~g}}, B_{1 \mathrm{~g}}$, and $B_{2 g}$. In contrast to the $J=1$ case, the $B_{1 g}$ and $B_{2 g}$ are $|1-\rangle$ and $|1+\rangle$. By using table 10 , we see that $A_{1 g}$ and $A_{2 g}$ arise from $| \pm 2\rangle$. In particular $A_{1 g}$ is $\mid 2+>$ while $A_{2 g}$ is $\mid 2->$. These may most conveniently be deduced from the transformation rules of table 8 , in comparison with the tesseral harmonics of table 2, keeping in mind the phase convention for odd positive $M$ of (2.8). The only nondiagonal matrix elements in the resulting secular determinant will be $\langle 0|V| 2+\rangle$, so that we have only one second degree equation to solve for the energies.

The polarization data are available only for the state at $+101 \mathrm{~cm}^{-1}$. This has polarization like the state $B_{1 g}$ or $B_{2 g}$ of $J=1$, and hence is presumably also $|1+\rangle$ or $\mid 1->$. We do not have any polarization data on the other levels, so we do not know which states are which. We are therefore forced to a trial-and-error process. The additional potential parameters significant for $J=2$ are $C_{4}^{0} C_{4}^{2} C_{4}^{4}$. If we select $\mid 1+>$ as 101 $\mathrm{cm}^{-1}$ and any one other as $|1-\rangle$, this will fix $C_{4}^{0}$ and $C_{4}^{2}$, so that only $C_{4}^{4}$ is left to fit the other three levels.

In attempting this process, the work is somewhat simplified by observing that $\left(\alpha 2|| Y_{2} \| \alpha 2\right)$ is very small, and that second degree terms will have only a small contribution to the matrix elements. Consequently, they were disregarded for the trial and error work, and were included later for a more exact calculation. The required matrix elements are, omitting the factor $\left(\alpha 2\left\|Y_{4}\right\| \alpha 2\right)$ common to all,

$$
\langle 0|V| 0\rangle=C_{00}^{242} Y_{4}^{0}\left\langle r^{4}\right\rangle
$$

$$
\begin{aligned}
& \langle 0|V| 2+\rangle=\frac{1}{\sqrt{2}}\left(C_{2-2}^{2}{ }^{42}+C_{-22}^{242}\right) Y_{4}^{2}\left\langle r^{4}\right\rangle \\
& =\frac{2}{\sqrt{2}} C_{-22}^{242} Y_{4}^{2}<r^{4}> \\
& \langle 2+|V| 2+\rangle=\frac{1}{2}\left(C_{20}^{242}+C_{-20}^{242}\right) Y_{4}^{0}\left\langle r^{4}\right\rangle \\
& +\frac{1}{2}\left(C_{-24}^{242}+C_{2-4}^{2-4}\right) Y_{4}^{4}<r^{4}> \\
& =C_{20}^{242} Y_{4}^{0}\left\langle r^{4}\right\rangle+C_{-24}^{242} Y_{4}^{4}\left\langle r^{4}\right\rangle \\
& \langle 1+|V| 1+\rangle=C_{10}^{242} Y_{4}^{0}\left\langle r^{4}\right\rangle+C_{-12}^{242} Y_{4}^{2}\left\langle r^{4}\right\rangle \\
& \langle 1-|V| 1-\rangle=C_{10}^{242} Y_{4}^{0}\left\langle r^{4}\right\rangle-C_{-12}^{242} Y_{4}^{2}\left\langle r^{4}\right\rangle \\
& \langle 2-|V| 2-\rangle=C_{20}^{242} Y_{4}^{0}\left\langle r^{4}\right\rangle-C_{-24}^{242} Y_{4}^{4}\left\langle r^{4}\right\rangle
\end{aligned}
$$

These may be simplified by observing that $C_{00}^{242}$ : $C_{10}^{242}: C_{20}^{242}=6:-4: 1$ and that $C_{22}^{242}=-\sqrt{3 / 8} C_{-22}^{242}$. If we introduce the following abbreviations,

$$
\begin{aligned}
\langle 0|V| 0\rangle & =V_{0} \quad<-2|V|+2>=\beta \\
& <-1|V|-1>=\delta
\end{aligned}
$$

the matrix elements may be simplified to:

$$
\begin{aligned}
<0|V| 0> & =V_{0} \\
<2+|V| 2+> & =\frac{1}{6} V_{0}+\beta \\
<2-|V| 2-> & =\frac{1}{6} V_{0}-\beta \\
<1+|V| 1+> & =-\frac{2}{3} V_{0}+\delta \\
<0|V| 2+> & =-\sqrt{\frac{3}{8}}(\sqrt{2}) \delta=-\frac{1}{2} \sqrt{3} \delta \\
<1-|V| 1-> & =-\frac{2}{3} V_{0}-\delta
\end{aligned}
$$

The form of these matrix elements suggests the physical situation. The $C_{4}^{0}$ term splits the $2 J+1$ levels into one at $V_{0}$, a pair at $-\frac{2}{3} V_{0}$ and a pair at $\frac{1}{6} V_{0}$. The first pair is then further split by $C_{4}^{2}$ into states separated by $2 \delta$. The second pair is also split into two separated by $2 \beta$ through $C_{4}^{4}$, but one of these interacts further (via $C_{4}^{2}$ ) with $|0\rangle$ through the nondiagonal matrix element $-\frac{1}{2} \sqrt{3} \delta$.

The trial and error fit was actually tried for all possible pairs of states as $|1+\rangle$ and $|1-\rangle$. The best fit was obtained for 101 (in agreement with the polarization data) and -75 . With this preliminary information, the second degree terms were included in the matrix elements, the fourth 
degree coefficients were chosen for a least squares fit in the energy, and the following were obtained:

\begin{tabular}{r|r|l}
\hline \hline$E$ (obs) & $E$ (calc) & \multicolumn{1}{|c}{ State } \\
\hline 101 & 108.0 & $|1+\rangle$ \\
62 & 65.9 & $2->$ \\
57 & 45.4 & $0.820|0\rangle+0.572 \mid 2+>$ \\
-75 & -85.8 & $|1->>| 2+>-0.572|0\rangle$ \\
-145 & -133.5 & $0.820 \mid 2+>$ \\
\hline
\end{tabular}

$$
\begin{aligned}
\left\langle r^{4}>Y_{4}^{0}=-187.8 \mathrm{~cm}^{-1}\right. & \left\langle r^{4}>Y_{4}^{2}=1,141 \mathrm{~cm}^{-1}\right. \\
& \left\langle r^{4}>Y_{4}^{4}=-626.2 \mathrm{~cm}^{-1} .\right.
\end{aligned}
$$

The fit is only fair. There are three possible sources of inaccuracy in the treatment. The first of these is the possibility of deviation from the $L-S$ coupling approximation. This would appear essentially in the values of the reduced matrix elements. Purely from the empirical standpoint, they could be treated as additional parameters to provide a better fit to the data. A second appreciable source of discrepancy is the interactions with other states, principally those within the ground multiplet. The nondiagonal Wigner coefficients and Racah coefficients are both generally of the same order of magnitude as the diagonal ones, and the reduced $L-S$ matrix elements of table 15 are appropriate for all combinations within the ground multiplet. Nondiagonal matrix elements can therefore be of appreciable magnitude and, in view of the relatively small multiplet splitting, could become quite important in a more refined theory. Finally, there is also the possibility that the symmetry is such as to require the inclusion of $S_{N}^{M}$ terms. This almost doubles the total number of parameters. This will, of course, permit an adjustment to the data, but there is no clear requirement for the inclusion of these terms. It may be noted here again that the earlier check on multiplet separation showed a distinct anomaly for the $J=2$ group.

Let us now turn our attention to $J=3$. Our allowed states are as follows:

$$
\begin{aligned}
& A_{2 g}: \mid 0>\text { and }\left|2+>A_{1 g}:\right| 2-> \\
& \quad B_{1 g}: \mid 3+>\text { and }\left|1+>B_{2 g}:\right| 1->\text { and } \mid 3->.
\end{aligned}
$$

If we compute the levels from the second and fourth degree terms only, we obtain a set of levels which show a rather surprisingly similar trend to the actually observed levels, which include the effect of sixth degree terms. Unfortunately, the polarization data both here and for still higher $J$ does not appear to follow either the rules appropriate for $\mathrm{D}_{2 h}$ symmetry found adequate for lower

\begin{tabular}{|c|c|c|}
\hline$E$ (obs) & $E$ (calc) & State \\
\hline $\begin{array}{r}37 \\
32 \\
27 \\
22 \\
-5 \\
-15 \\
-98\end{array}$ & $\begin{array}{r}45.1 \\
36.9 \\
21.8 \\
8.6 \\
-14.8 \\
-28.0 \\
-69.6\end{array}$ & 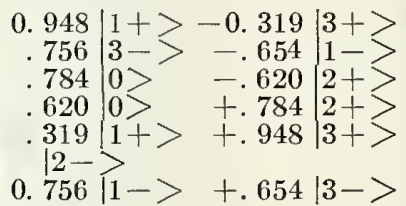 \\
\hline
\end{tabular}
$J$, or any other pattern easily amenable to analysis. In particular, the states given do not especially even suggest the reported polarization data.

There is obviously a considerable amount of work yet to be done before the $\mathrm{Tb}$ spectrum is understood. Experimentally, there is need for more attention to polarization data for fixing the character of the states involved. Theoretically, there are several lines of endeavor to be pursued. The problems become numerically so tedious that machine calculation is probably required for further success. The first of these is a systematic machine calculation of nondiagonal Wigner coefficients. The published table of Simon (1954) is inadequate in range, and the extension of table 15 by desk calculations is almost out of the question. For the most part, the tables of the Racah coefficients (Simon et al., 1954) are adequate, so that the availability of Wigner coefficients would permit a study of nondiagonal contributions to the energies. Furthermore, it appears that in problems of low symmetry, such as the present one, there will be a requirement for trial-and-error calculations. These are well adapted to machine calculations, but are again, except in very simple cases, almost out of the question for desk calculators.

\section{Bibliography}

1905

Kayser, H. G. J., Handbuch der Spectroscopie 3, 427 (S. Hirzel).

1906

Frobenius, G. and Schur, I., Uber die reellen Darstellungen der endlichen Gruppen, Sitzber. preuss. Akad Wiss., Physik-math. Kl. I, 186.

\section{8}

Becquerel, J., Recherches sur les phenomenes magnetooptiques dans les cristaux et les solutions solidifiees a la temperature de l'air liquide Radium $\mathbf{V}, 5$.

Becquerel, J. and Onnes, H. K., The absorption spectra of the compounds of the rare earths at the temperatures obtainable with liquid hydrogen, and their change by the magnetic field, Koninkl. Akad. Wetenschap. (Amsterdam) 10,592 .

Tallqvist, H., Tafeln der Kugelfunctionen, Acta Soc. Sci. Fennicae 33, No. 4. (a) Tafeln der Abgeleiteten und Zugeordneten Kugelfunctionen Erster Art, Acta Soc. Sci. Fennicae 33, No. 9.

du Bois, H. and Elias, G. J., Der Einfluss von Temperatur and Magnetisierung bei selektiven Absorptions- und Fluoreszenzspektren, Ann. Physik 4.27, 233.

1911

du Bois, H. and Elias, G. J., Der Einfluss von Temperatur und Magnetisierung bei selektiven Absorptions- und Fluoreszenzspektren, Ann. Physik 4.35, 617. 
1923

Schoenflies, A. Theorie der Kristallstruktur. Berlin, Gebrüder Borntraeger.

1925

Hund, F., Atomtheoretische Deutung des Magnetismus der Seltenen Erden, Z. Physik 33, 855.

1926

Becquerel, J., Onnes, H. K., and de Haas, W. J., The absorption bands of the compounds of the rare earths, their modification by a magnetic field, and the magnetic rotation of the plane of polarisation at very low temperatures, Koninkl. Akad. Wetenschap. (Amsterdam) 29, 264.

1928

Weyl, H., Gruppentheorie und Quantenmehanik (S. Hirzel).

1929

Bethe, H., Termaufspaltung in Kristallen, Ann. Physik 5.3, 133. [Splitting of Terms in Crystals, Consultants Bureau, Tr.]

Brunetti, R. and Ollano, Z., L'Azione del campo elettrico sulle radiazioni degli joni di terre rare in composto chimico, Nuovo cimento 6,345 .

Freed, S. and Spedding, F. H., Line absorption spectra in solids at low temperatures in the visible and ultraviolet regions of the spectrum, Nature 123, 525 .

Kramers, M. H. A., La rotation paramagnétique du plan de polarisation dans les cristaux uniaxes de terres rares, Koninkl. Akad. Wetenschap. (Amsterdam) 32, 1176.

Van Vleck, J. H. and Frank, A., The effect of second order Zeeman terms on magnetic susceptibilities in the rare earth and iron groups, Phys. Rev. 34, 1494.

1930

Bethe, H., Zur Theorie des Zeemaneffektes an den Salzen der seltenen Erden, Z. Physik 60, 218.

Eckart, C., The application of group theory to the quantum dynamics of monatomic systems, Rev. Modern Phys. 2, 305.

Kramers, H. A., Theorie generale de la rotation paramagnetique dans les cristaux, Koninkl. Akad. Wetenschap. (Amsterdam) 33, 959.

Wigner, E., Uber die elastischen Eigenschwingungen symmetriseher Systeme, Nachr. Akad. Wiss. Gottingen, Math.-physik. Kl. 2, 133.

1931

Spedding, F. H., Interpretation of the spectra of rare earth crystals, Phys. Rev, 37, 777.

Wigner, E., Gruppentheorie (F. Vieweg und Sohn).

1932

Penney, W. G. and Schlapp, R., Influence of crystalline fields on the spectra of salts of paramagnetic ions. I. The rare earths, especially Pr and Nd, Phys. Rev, 41, 194.

Schlapp, R. and Penney, W. G., Influence of crystalline fields on the susceptibilities of salts of paramagnetic ions. II. The iron group, especially $\mathrm{Ni}, \mathrm{Cr}$, and $\mathrm{Co}$, Phys. Rev. 42, 666.

Wigner, E., Uber die Operation der Zeitumkehr in der Quantenmechanik, Nachr. Akad. Wiss. Gottingen, Math.-physik. Kl, 5, 546.

Spedding, F. H. and Bear, R. S., Absorption spectrum of the samarium ion in solids. I. Absorption by large single crystals of $\mathrm{SmCl}_{3} \cdot 6 \mathrm{H}_{2} \mathrm{O}$, Phys. Rev. 42, 58 .

Van Vleck, J. H. Theory of electric and magnetic susceptibilities (Oxford University Press, Oxford, England).

1933

Spedding, F. H., Zeernan effect in solids, J. Chem. Phys. 1,144 .

1935

Condon, E. U. and Shortley, G. H., Theory of atomic spectra (Cambridge University Press, England).
Lang, R. J., The spectrum of trebly ionized cerium, Can. J. Research 14A, 127.

1937

Ahlberg, J. E., Blanchard, E. R., and Lundberg, W. O.,

Electronic activation in crystals: The heat capacities of

$\mathrm{Sm}_{2}\left(\mathrm{SO}_{4}\right)_{3} \cdot 8 \mathrm{H}_{2} \mathrm{O}$ and $\mathrm{Nd}_{2}\left(\mathrm{SO}_{4}\right)_{3} \cdot 8 \mathrm{H}_{2} \mathrm{O}$ from 3 to $40^{\circ} \mathrm{K}$,

J. Chem. Phys. 5, 552.

Van Vleck, J. H., The puzzle of rare earth spectra in solids,

J. Phys. Chem. 41, 67.

1938

Murnaghan, F. D., Theory of group representations (Johns Hopkins Press, Baltimore, Md.).

1940

Opechowski, W., Sur les groupes cristallographiques

"Doubles", Physica 7, 552.

1941

Stratton, J. A., Electromagnetic theory (McGraw Hill Book Co., Inc., New York, N.Y.), pg. 408.

1942

Racah, G., Theory of complex spectra I, Phys. Rev. 61, 186. (a) Theory of complex spectra II, Phys. Rev. 62,

438. (b) Theory of complex spectra III, Phys. Rev. 63, 367 (1943).

1945

U.S. National Bureau of Standards, Tables of associated Legendre functions (Columbia University Press, New York, N.Y.).

1948

Hellwege, K. H., Elektronenterme und Strahlung von Atomen in Kristallen. I. Termaufspaltung und elektrische Dipolstrahlung, Ann. Physik 6.4, 97. (a) Elektronenterme und Strahlung von Atomen in Kristallen. II. Magnetische Dipolstrahlung, Ann. Physik 6.4, 127. (b) Elektronenterme und Strahlung von Atomen in Kristallen. III. Elektrische Quadrupolstrahlung, Ann. Physik 6.4, 136 . (c) Elektronenterme und Strahlung von Atomen in Kristallen. IV. Symmetrieentartung und Kramerssche Entartung 6.4, 143. (d) Elektronenterme und Strahlung von Atomen in Kristallen. V. Kubisehe Kristalle, Ann. Physik 6.4, 150.

1949

Racah, G., Theory of complex spectra IV, Phys. Rev. 76, 1352.

1952

Biedenharn, L. C., Tables of the Racah Coefficients, Oak Ridge Nat'l Lab. Rep. 1098.

Falkoff, D. L., Colladay, G. S., and Sells, R. E., Transformation amplitudes for vector addition of angular momentum $\left(j 3 m^{\prime} \mid j 3 J M\right)$, Can. J. Phys. 30, 253.

Klein, M. J., On a degeneracy theorem of Kramers, Am. J. Phys. 20, 65.

Stevens, K. W. H., Matrix elements and operator equivalents connected with the magnetic properties of rare earth ions, Proc. Phys. Soc. (London) A.65, 209.

1953

Bleaney, B. and Stevens, K. W. H., Paramagnetic resonance, Repts. Prog. in Phys. 16, 108.

Elliott, R. J. and Stevens, K. W. H., The theory of magnetic resonance experiments on salts of the rare earths, Proc. Roy. Soc. (London) A218, 553. (a) The magnetic properties of certain rare earth ethyl sulphates, Proc. Roy. Soc. (London) A219, 387.

1954

Simon, A., A numerical table of the Clebsch-Gordan coefficients, Oak Ridge Nat'l Rep. 1718.

Simon, A., Vander Sluis, J. H., and Biedenharn, L. C., Tables of the Racah coefficients, Oak Ridge Nat'l Rep. 1679. 
Bowers, K. D. and Owen, J., Paramagnetic resonance. II., Repts. Prog. in Phys. 18, 304.

Judd, R. B., Operator equivalents and matrix elements for the excited states of rare earth ions, Proc. Roy. Soc. (London) A.227, 552. (a) The theory of the spectra of europium salts, Proc. Roy. Soc. (London) A.228, 120 .

Mulliken, R. S. Report on notation for the spectra of polyatomic molecules, J. Chem. Phys. 23, 1997.

\section{6}

Dieke, G. H. and Crosswhite, H. M., Absorption spectrum and magnetic properties of ytterbium chloride, J. Opt. Soc. Am. 46, 885 .

Dieke, G. H. and Heroux, L., Absorption spectra, Zeeman effects, and magnetic properties of neodymium salts. Phys. Rev. 103, 1227.

Melvin, M. A., Simplification in finding symmetryadapted eigenfunctions, Rev. Mod. Phys. 28, 18.

\section{Washington, January 15,1960}

Edmonds, A. R., Angular momentum in quantum mechanics (Princeton University Press, Princeton, N.J.).

Fick, E. and Joos, G., Kristallspektren, Handbuch der Physik 28, 205.

Rose, M. E., Elementary theory of angular momentum (John Wiley \& Sons, Inc., 440 Fourth Ave., New York, N.Y.).

Singh, Shobha, Absorption, fluorescence, and Zeeman effects of dysprosium, terbium, holmium and uranyl compounds at low temperatures, Dissertation, Johns Hopkins $\mathrm{U}$.

\section{8}

Low, W., Paramagnetic resonance spectrum of trivalent gadolinium in the cubic field of calcium fluoride, Phys. Rev. 109, 265.

\section{0}

Herzfeld, C. M. and Meijer, P. H. E., Group theory and crystal field theory (to be published by Academic Press, Inc., New York, New York). 


\section{THE NATIONAL BUREAU OF STANDARDS}

The scope of activities of the National Bureau of Standards at its major lahoratories in Washington, D.C., and Boulder, Colorado, is suggested in the following listing of the divisions and sections engaged in technical work. In general, each section carries out specialized research, development, and engineering in the field indicated hy its title. A hrief description of the activities, and of the resultant pullications, appears on the inside of the front cover.

\section{WASHINGTON, D.C.}

Electricity and Electronics. Resistance and Reactance. Electron Devices. Electrical Instruments. Magnetic Measurements. Dielectrics. Engineering Electronics. Electronic Instrumentation. Electrochemistry.

Optics and Metrology. Photometry and Colorimetry. Optical Instruments. Photographic Technology. Length. Engineering Metrology.

Heat. Temperature Physics. Thermodynamics. Cryogenic Physics. Rheology. Molecular Kinetics. Free Radicals Research.

Atomic Physics. Spectrosenpy. Radiometry. Mass Spectrometry. Solid State Physics. Electron Physics. Atomic Physics.

Radiation Physics. Neutron Physics. Radiation Theory. Radioactivity. X-ray. High Energy Radiation. Nucleonic Instrumentation. Radiological Equipment.

Chemistry. Organic Coatings. Surface Chemistry. Organic Chemistry. Analytical Chemistry. Inorganic Chemistry. Electrodeposition. Molecular Structure and Properties of Gases. Physical Chemistry. Thermochemistry. Spectrochemistry. Pure Suhstances.

Mechanics. Sound. Mechanical Instruments. Fluid Mechanics. Engineering Mechanics. Mass and Scale. Capacity, Density, and Fluid Meters. Comhustion Controls.

Organic and Fibrous Materials. Ruhher. Textiles. Paper. Leather. Testing and Specifications. Polymer Structure. Plastics. Dental Research.

Metallurgy. Thermal Metallurgy. Chemical Metallurgy. Mechanical Metallurgy. Corrosion. Metal Physics.

Mineral Products. Engineering Ceramics. Glass. Refractories. Enameled Metals. Constitution and Microstructure.

Building Technology. Structural Engineering. Fire Protection. Air Conditioning, Heating, and Refrigeration. Floor, Roof, and $W$ all Coverings. Codes and Safety Standards. Heat Transfer. Concreting Materials.

Applied Mathematics. Numerical Analysis. Computation. Statistical Engineering. Mathematical Physics.

Data Processing Systems. SEAC Engineering Group. Components and Techniques. Digital Circuitry. Digital Systems. Analog Systems. Applications Engineering.

- Office of Basic Instrumentation.

- Office of Weights and Measures.

\section{BOULDER, COLORADO}

Cryogenic Engineering. Cryogenic Equipment. Cryogenic Processes. Properties of Materials. Gras Liquefaction. Radio Propagalion Physics. Upper Atmosphere Research. Ionosphere Research. Regular Prediction Services. Sun-Earth Relationships. VHF Research. Radio Warning Services. Airglow and Aurora. Radio Astronomy and Arctic Propagation.

Radio Propagation Engineering. Data Reduction Instrumentation. Radio Noise. Tropospberic Measurements. Tropospheric Analysis. Propagation-Terrain Effects. Radio-Meteorology. Lower Atmosphere Physics.

Radio Standards. High-Frequency Electrical Standards. Radio Broadcast Service. Radio and Microwave Materials. Atomic Frequency and Time Standards. Electronic Calibration Center. Microwave Circuit Standards.

Radio Communication and Systems. Low Frequency and Very Low Frequency Research. High Frequency and Very High Frequency Research. Modulation Systems. Antenna Research. Navigation Systems. Systems Analysis. Field Operations. 
